\title{
ARL STATISTICS 2007-2008
}

Compiled and Edited by

MARTHA KYRILLIDOU

LES BLAND

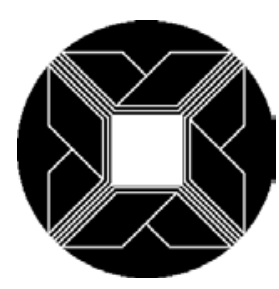

Association of ResEARCH LibRARIES

WASHINGTON, DC

2009 
ARL Statistics 2007-2008

The tables presented in this publication are not indicative of performance and outcomes and should not be used as measures of library quality. In comparing any individual library to ARL medians or to other ARL members, one must be careful to make such comparisons within the context of differing institutional and local goals and characteristics.

The ARL Statistics datafiles and accompanying documentation are available at www.arl.org/stats / annualsurveys / arlstats / index.shtml.

Published by the

Association of Research Libraries

Washington, DC 20036

www.arl.org

ISSN 0147-2135 print

ISSN 1943-5983 online

ISBN 1-59407-845-9

EAN 978-159407-845-3

\section{(C) 2009}

The compilation is copyrighted by the Association of Research Libraries. Blanket permission is granted to reproduce and distribute copies of this work for nonprofit, educational, or library purposes, provided that the author, source, and copyright notice are included on each copy. This permission is in addition to rights of reproduction granted under Sections 107,108 , and other provisions of the US Copyright Act.

The paper used in this publication meets the minimum requirements of the American National Standard for Information Science and National Information Standards Organization standard-Permanence of Paper for Publications and Documents in Libraries and Archives, ANSI/NISO Z39.48-1992(R1997). 


\section{CONTENTS}

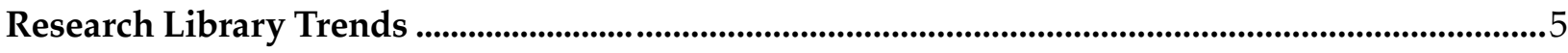

\section{Research Library Trends Tables}

1. Service Trends in ARL Libraries, 1991-2008 ………………................................................................................

2. Median Monograph and Serial Expenditures in ARL Libraries, 1986-2008..........................................10

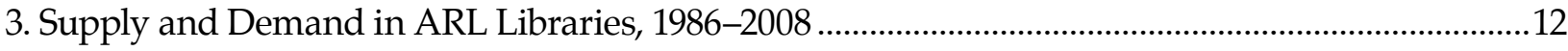

4. Expenditure Trends in ARL Libraries, 1986-2008 ………………………………………………………...14

5. Resources per Student in ARL University Libraries, 1986-2008 ……….....................................................16

6. Electronic Resources Expenditures in ARL University Libraries, 2007-2008 ...................................18

7a. Electronic Resources and Materials Expenditures in ARL University Libraries, 1992-2002 .........20

7b. Electronic Resources and Materials Expenditures in ARL University Libraries, 2002-2008.........21

\section{Research Library Trends Graphs}

1. Service Trends in ARL Libraries, 1991-2008 ...................................................................................................9

2. Median Monograph and Serial Expenditures in ARL Libraries, 1986-2008............................................11

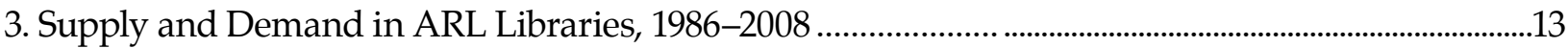

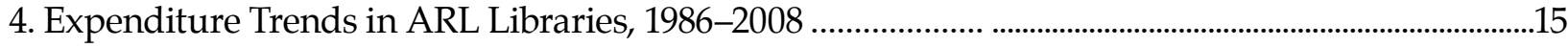

5. Resources per Student in ARL University Libraries, 1986-2008 ……………………………………….....17

6. Electronic Resources vs. Total Materials Expenditures, 1993-2008 ……................................................19

ARL Statistics Interactive Edition .............................................................................................................22

ARL Library Data Tables, 2007-2008

Collections: Volumes and Monographs...........................................................................................................2

Summary Data …………………………………………………………………………………………....

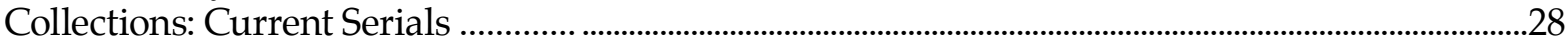

Summary Data ……………………………………………………………………………………………34

Collections: Other Materials ........................................................................................................................................36

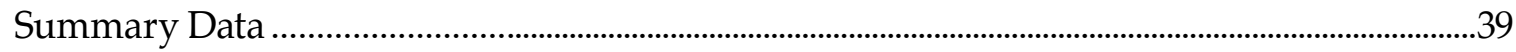

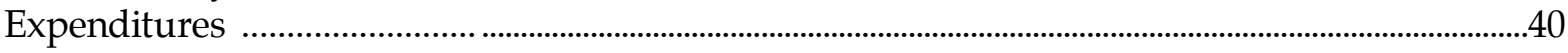

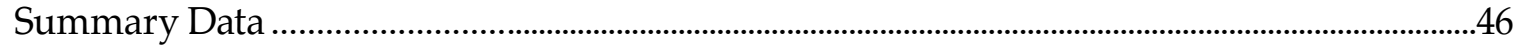

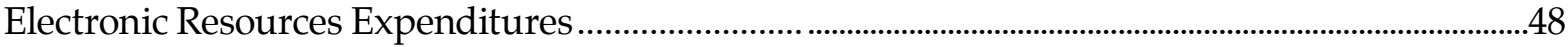

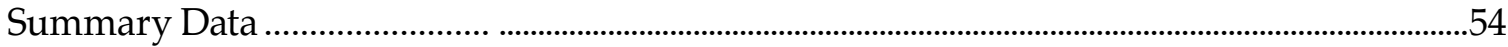

Personnel and Public Services ..............................................................................................................................56

Summary Data ………………………………………………………………………………………………..62

Analysis of Selected Variables of University Libraries, 2007-2008 ...................................................64 
PhD, Faculty, and Enrollment Statistics. 65

Summary Data

Rank Order Tables of University Libraries, 2007-2008

Summary of Rank Order Tables ..............................................................................................................

Individual Tables

1. Volumes in Library ...................................................................................................................................74

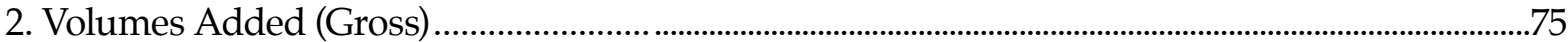

3. Current Serials (Total) .......................................................................................................................76

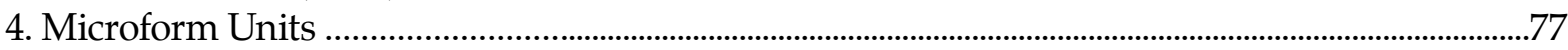

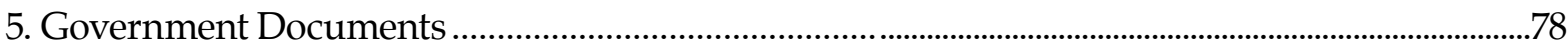

6. Total Library Materials Expenditures …….............................................................................................79

7. Total Salaries \& Wages Expenditures ..............................................................................................................80

8. Other Operating Expenditures …........................................................................................................8

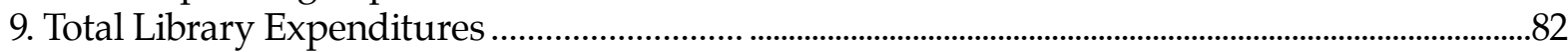

10. Monographs Purchased (Volumes) .........................................................................................................83

11. Expenditures for Monographs................................................................................................................84

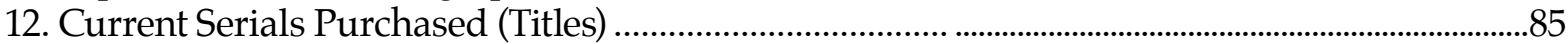

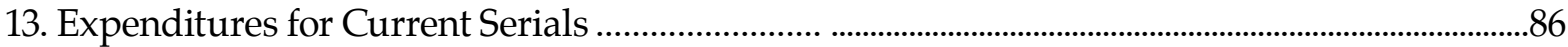

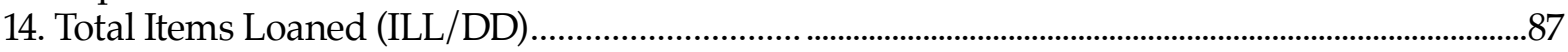

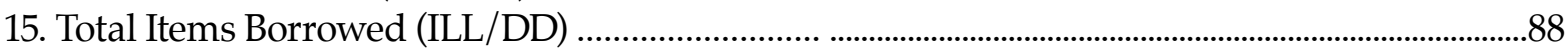

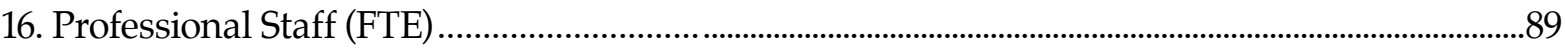

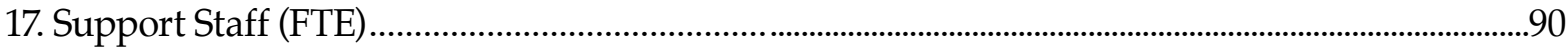

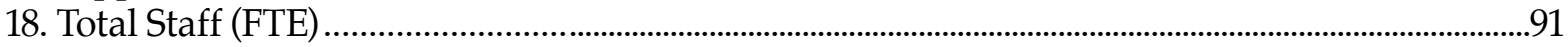

19. Expenditures for Electronic Resources …...................................................................................92

20. Electronic Resources as a Percent of Total Library Materials............................................................93

21. Library Investment Index ..........................................................................................................94

ARL Statistics Questionnaire, 2007-2008: Instructions for Completing the Questionnaire.............95

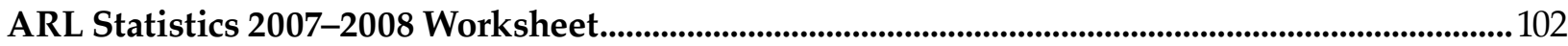

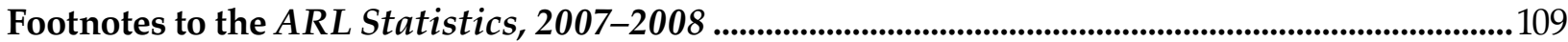

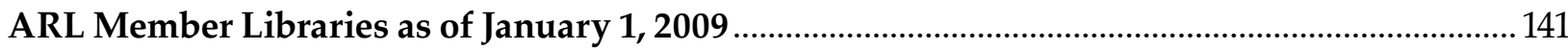

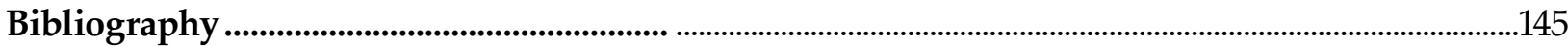




\section{Research Library TrendS}

ARL Statistics 2007-2008 is the latest in a series of annual publications that describe collections, staffing, expenditures, and service activities for the 123 members of the Association of Research Libraries (ARL). Of these, 113 are university libraries; the remaining 10 are public, governmental, and nonprofit research libraries. ARL member libraries are the largest research libraries in North America, representing 16 Canadian and 107 US research institutions. The academic libraries, which comprise about $92 \%$ of the membership, include 14 Canadian and 99 US libraries.

Statistics have been collected and published annually for the members of the Association of Research Libraries since 1961-1962, and the data are available through an interactive Web interface. Prior to 1961-1962, annual statistics for university libraries were collected by James Gerould, first at the University of Minnesota and later at Princeton University. These data, covering the years 1907-1908 through 1961-1962, are now called the Gerould statistics. ${ }^{2}$ The whole data series from 1908, which is available on the ARL server, ${ }^{3}$ represents the oldest and most comprehensive continuing library statistical series in North America.

ARL libraries are a relatively small subset of libraries in North America, but they do account for a large portion of academic library resources in terms of assets, budgets, and the number of users they serve. The total library expenditures of all 123 member libraries in 2007-2008 was more than $\$ 4$ billion; from that, roughly $\$ 3$ billion was spent by the 113 university libraries and more than $\$ 1$ billion by the nonuniversity libraries. The pie charts below show how the two types of libraries divide these expenditures differently.

University Libraries 2007-2008

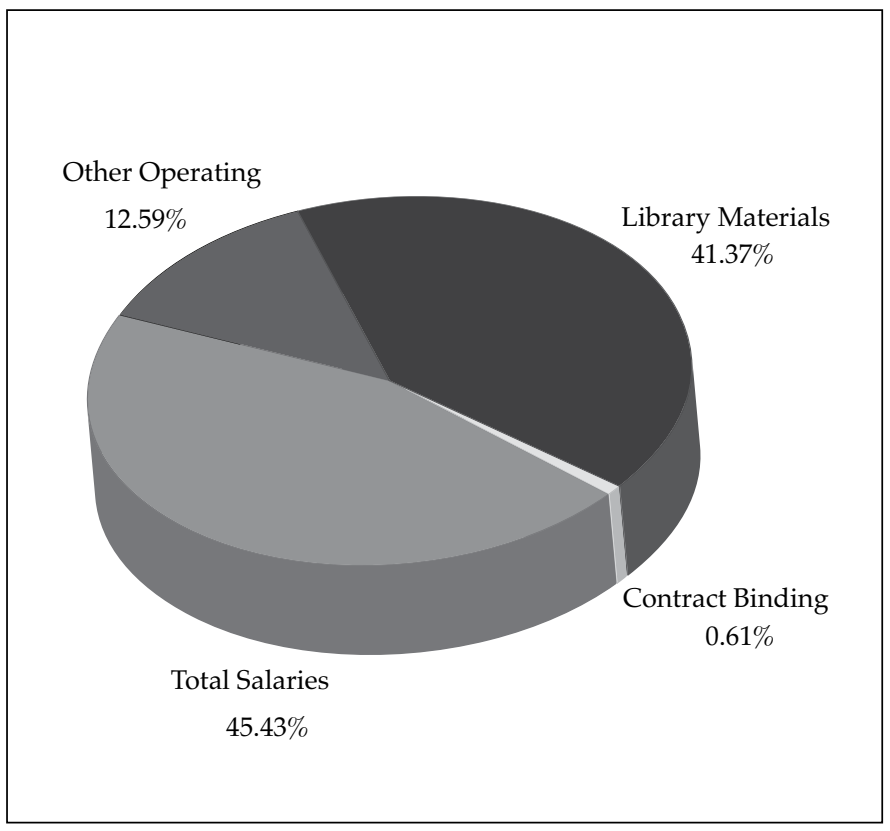

Nonuniversity Libraries 2007-2008

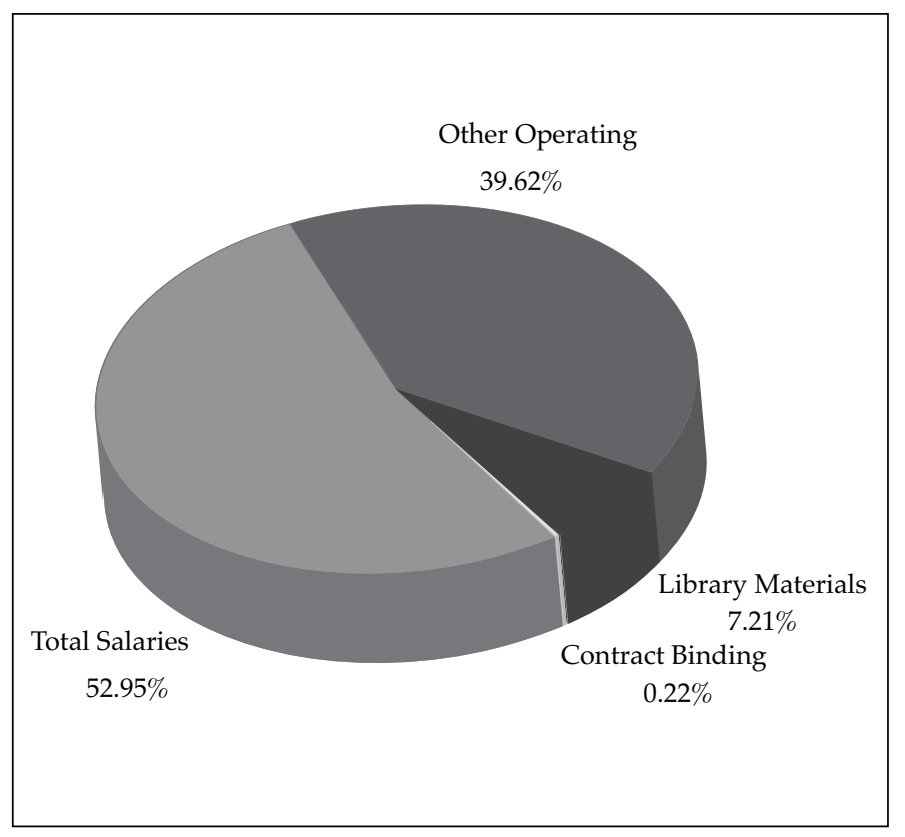

\footnotetext{
${ }^{1}$ Kendon L. Stubbs and Robert E. Molyneux, Research Library Statistics 1907-08 through 1987-88 (Washington, DC: ARL, 1990).

2 Robert E. Molyneux, The Gerould Statistics 1907/08 - 1961/62. (Washington, DC: ARL, 1986) http:/ / fisher.lib.virginia.edu/gerould/.

3 http: / / www.arl.org/stats/arlstat/mrstat.html.
} 


\section{Change in Counting Serials}

For the second year in a row the definition of counting serials in the ARL Statistics is now counting serial titles. As a result the trend line of publishing serial cost per subscription is being discontinued and eventually a new one will emerge as we collect annual data on serial titles. This change, though simple at first glance, is very important as it makes the concept of serials more meaningful in the electronic environment.

What prompted this change? In earlier years the instructions for reporting serials were that libraries report the "total number of subscriptions, not titles, but electronic serials acquired as part of an aggregated package (such as MUSE or Academic's IDEAL) were to be counted by title." Directors and other staff have expressed concern that the serials count was problematic since many libraries engage in multiple consortia arrangements and the serials count was inflated by duplicate titles held in multiple packages. The Statistics and Assessment Committee determined that a new way of counting serials focusing on titles would provide better descriptive data reflecting the true scope of the content provided by research libraries. A pilot at various ARL libraries demonstrated the feasibility of the new method.

In the electronic environment once you own or lease a title, it is often accessible by all users so a more authentic descriptive statistic for the scope of the content is titles rather than subscriptions. A unique title count favors broader coverage. Any duplication of those titles through packages, aggregations, bundles, etc., becomes more a management issue. The new definition asks that titles be reported as electronic if available both in print and electronic formats and they be reported as purchased if available both through purchased and non-purchased arrangements. So, if a title appears in both print and electronic form and a library has acquired it through several different providers, it would be counted as one title. Training events were delivered last fall to ensure that there is a well-grounded, shared understanding of the new definitions.

The outcome has been successful as we have more libraries reporting serials titles than they ever managed to report serial subscriptions indicating that the new definition is moving us into the right direction of having data that are both collectable and useful. However, we had to delete all earlier trend lines that were based on serial subscriptions. A variety of resources have been developed for libraries to consult as they implemented the change and they are available through http://www.arl.org/stats/annualsurveys/arlstats/07statmail.shtml.

This year, 2007-2008, we changed the categories of serials reported under "serials titles currently received but not purchased." The subcategories are now: (a) consortial, (b) freely accessible, (c) print (and other formats) - exchanges, gifts, etc., and (d) government documents. These categories are more meaningful in the context of "serials titles received" as they emphasize major components of that concept. 


\section{Research Library Trends Tables and GraphS}




\section{TABLE 1}

\section{Service Trends in ARL Libraries, 1991-2008 Median Values for Time-Series Trends}

\begin{tabular}{|c|c|c|c|c|c|c|c|c|c|}
\hline Year & $\begin{array}{r}\text { ILL: } \\
\text { Borrowed }\end{array}$ & $\begin{array}{r}\text { Group } \\
\text { Pres. }\end{array}$ & $\begin{array}{r}\text { Participants } \\
\text { In Pres. }\end{array}$ & $\begin{array}{r}\text { Reference } \\
\text { Trans. }\end{array}$ & $\begin{array}{r}\text { Initial } \\
\text { Circ. }\end{array}$ & $\begin{array}{l}\text { Total } \\
\text { Circ. }\end{array}$ & $\begin{array}{r}\text { Ratio of Circ. } \\
\text { Init./Tot. }\end{array}$ & $\begin{array}{l}\text { Total } \\
\text { Staff } \\
\end{array}$ & $\begin{array}{r}\text { Total } \\
\text { Students }\end{array}$ \\
\hline (Libraries) & (103) & (84) & (82) & (79) & (36) & (80) & (34) & (105) & (103) \\
\hline 1991 & 10,397 & 508 & 7,137 & 125,103 & 296,964 & 509,673 & 1.26 & 271 & 18,290 \\
\hline 1992 & 11,362 & 526 & 7,154 & 132,549 & 342,989 & 554,579 & 1.27 & 265 & 18,273 \\
\hline 1993 & 12,489 & 616 & 7,688 & 136,115 & 343,293 & 568,628 & 1.32 & 262 & 18,450 \\
\hline 1994 & 14,007 & 568 & 7,831 & 147,582 & 369,996 & 572,749 & 1.31 & 264 & 18,305 \\
\hline 1995 & 14,472 & 687 & 8,461 & 147,023 & 347,144 & 578,989 & 1.32 & 267 & 18,209 \\
\hline 1996 & 15,278 & 719 & 8,410 & 155,336 & 336,481 & 560,244 & 1.39 & 264 & 18,320 \\
\hline 1997 & 16,264 & 687 & 9,218 & 149,659 & 348,157 & 542,438 & 1.37 & 273 & 18,166 \\
\hline 1998 & 17,656 & 698 & 9,462 & 132,850 & 354,924 & 514,574 & 1.37 & 273 & 18,335 \\
\hline 1999 & 18,942 & 711 & 9,406 & 128,696 & 300,923 & 514,087 & 1.38 & 277 & 18,609 \\
\hline 2000 & 20,475 & 722 & 9,596 & 115,636 & 273,231 & 482,542 & 1.42 & 267 & 18,908 \\
\hline 2001 & 21,902 & 669 & 10,121 & 104,409 & 265,195 & 467,277 & 1.48 & 269 & 19,102 \\
\hline 2002 & 21,339 & 776 & 11,350 & 95,910 & 251,146 & 462,223 & 1.51 & 279 & 19,925 \\
\hline 2003 & 22,146 & 806 & 12,516 & 89,150 & 248,689 & 479,733 & 1.57 & 277 & 21,132 \\
\hline 2004 & 25,737 & 757 & 12,864 & 84,546 & 261,526 & 496,369 & 1.60 & 273 & 21,562 \\
\hline 2005 & 25,729 & 803 & 13,782 & 65,168 & 250,971 & 473,216 & 1.58 & 267 & 22,047 \\
\hline 2006 & 27,412 & 833 & 13,051 & 67,697 & 267,213 & 466,403 & 1.52 & 267 & 22,618 \\
\hline 2007 & 26,813 & 830 & 14,417 & 61,703 & 222,037 & 456,597 & 1.59 & 266 & 22,874 \\
\hline 2008 & 27,822 & 803 & 15,480 & 58,763 & 221,144 & 429,626 & 1.59 & 260 & 22,762 \\
\hline $\begin{array}{l}\text { Average annual } \\
\% \text { change }\end{array}$ & $6.0 \%$ & $2.7 \%$ & $4.7 \%$ & $-4.3 \%$ & -1.7 & -1.0 & $1.4 \%$ & -0.2 & $1.3 \%$ \\
\hline
\end{tabular}

Table 1 presents data about select public service activities such as circulation (initial and total), reference transactions, library instruction (group presentations and participants in these presentations), and interlibrary borrowing and lending. As seen in table 1, the median of group presentations dropped again during 2007-2008. However, the number of participants increased with a median of 15,480 for the typical research library. Perhaps what is of most interest is that by 2008 there were more than double the participants compared to 1991, while there is only a 20 percent difference in the number of students between 1991 and 2008 (see Graph 1). 


\section{GRAPH 1}

\section{Service Trends in ARL Libraries, 1991-2008}

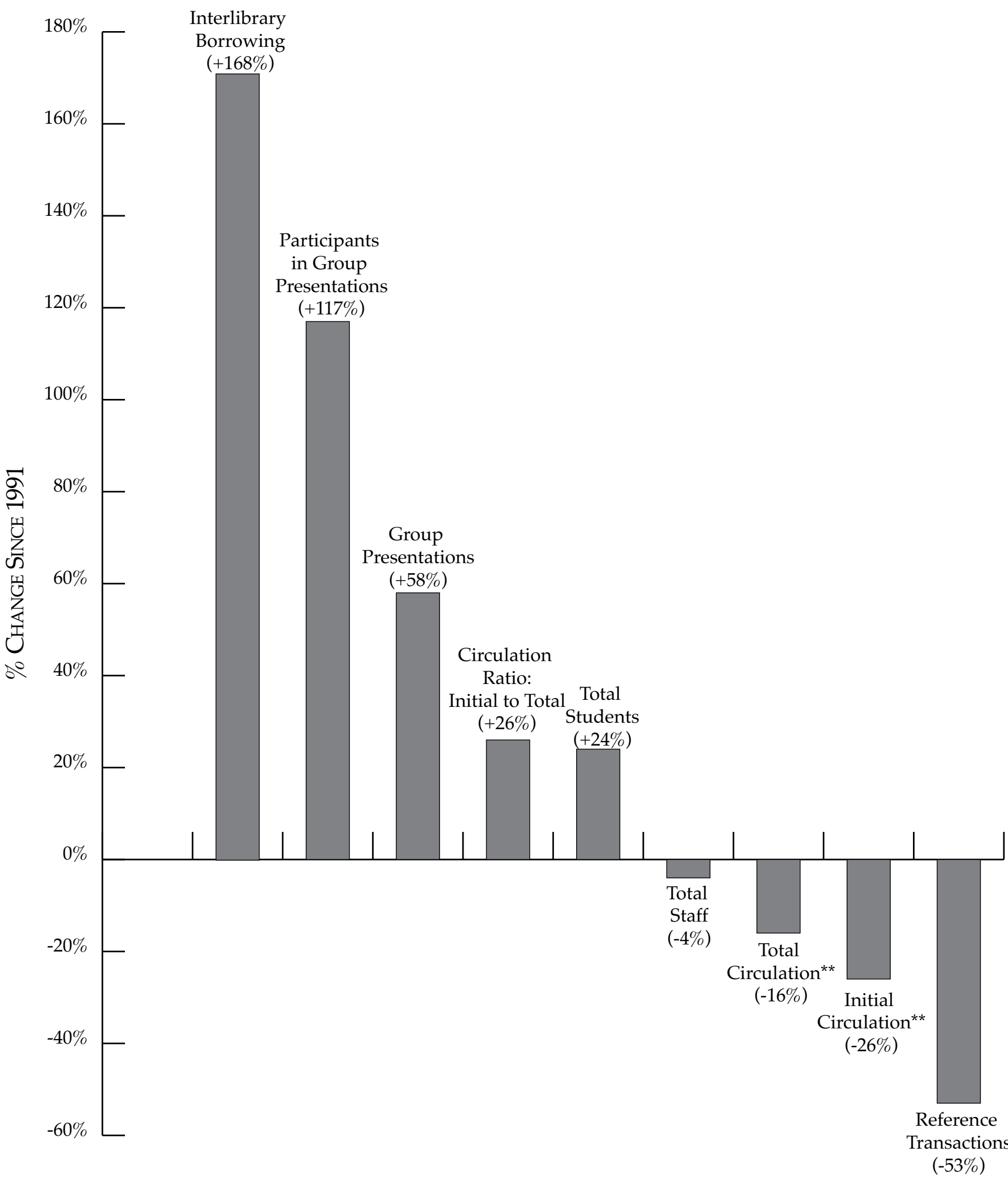

** Total Circulation includes Initial and Renewal but excludes Reserve Circulation.

Source: ARL Statistics 2007-2008, Association of Research Libraries, Washington, DC 
TABLE 2

\section{Median Monograph and Serial Expenditures in ARL Libraries, 1986-2008 Median Values for Time-Series Trends}

\begin{tabular}{|c|c|c|c|c|}
\hline Year & $\begin{array}{r}\text { Serial } \\
\text { Expenditures } \\
\end{array}$ & $\begin{array}{r}\text { Monograph } \\
\text { Unit Cost }\end{array}$ & $\begin{array}{r}\text { Monograph } \\
\text { Expenditures }\end{array}$ & $\begin{array}{r}\text { Monographs } \\
\text { Purchased }\end{array}$ \\
\hline (Libraries) & (100) & (57) & (97) & (58) \\
\hline 1986 & $\$ 1,496,775$ & \$ 29.28 & $\$ 1,120,645$ & 32,679 \\
\hline 1987 & $\$ 1,769,960$ & $\$ 31.76$ & $\$ 1,064,484$ & 26,240 \\
\hline 1988 & $\$ 1,947,559$ & $\$ 36.03$ & $\$ 1,141,226$ & 25,238 \\
\hline 1989 & $\$ 2,113,976$ & $\$ 38.44$ & $\$ 1,106,073$ & 27,082 \\
\hline 1990 & $\$ 2,296,910$ & \$ 41.44 & $\$ 1,330,747$ & 27,546 \\
\hline 1991 & $\$ 2,548,687$ & $\$ 42.60$ & $\$ 1,400,738$ & 27,843 \\
\hline 1992 & $\$ 2,620,832$ & $\$ 44.14$ & $\$ 1,353,865$ & 26,947 \\
\hline 1993 & $\$ 2,918,569$ & $\$ 43.74$ & $\$ 1,295,807$ & 25,188 \\
\hline 1994 & $\$ 2,912,495$ & $\$ 44.72$ & $\$ 1,309,807$ & 25,341 \\
\hline 1995 & $\$ 3,131,033$ & $\$ 45.27$ & $\$ 1,365,575$ & 25,707 \\
\hline 1996 & $\$ 3,389,118$ & $\$ 46.73$ & $\$ 1,444,015$ & 25,911 \\
\hline 1997 & $\$ 3,642,541$ & \$ 46.42 & $\$ 1,460,234$ & 28,576 \\
\hline 1998 & $\$ 3,816,497$ & $\$ 47.59$ & $\$ 1,486,764$ & 24,447 \\
\hline 1999 & $\$ 4,095,934$ & $\$ 47.78$ & $\$ 1,506,651$ & 25,173 \\
\hline 2000 & $\$ 4,430,812$ & $\$ 47.87$ & $\$ 1,657,349$ & 28,024 \\
\hline 2001 & $\$ 4,660,349$ & $\$ 48.48$ & $\$ 1,864,023$ & 30,494 \\
\hline 2002 & $\$ 4,939,225$ & $\$ 50.61$ & $\$ 1,812,826$ & 31,578 \\
\hline 2003 & $\$ 5,392,007$ & $\$ 52.83$ & $\$ 1,873,157$ & 33,208 \\
\hline 2004 & $\$ 5,580,157$ & $\$ 51.36$ & $\$ 1,839,412$ & 30,155 \\
\hline 2005 & $\$ 5,962,446$ & $\$ 53.57$ & $\$ 1,784,841$ & 30,546 \\
\hline 2006 & $\$ 6,307,292$ & $\$ 52.04$ & $\$ 2,044,272$ & 33,145 \\
\hline 2007 & $\$ 6,587,241$ & $\$ 54.17$ & $\$ 2,097,078$ & 36,462 \\
\hline 2008 & $\$ 7,097,140$ & $\$ 55.41$ & $\$ 2,084,991$ & 32,745 \\
\hline $\begin{array}{l}\text { Average annual } \\
\% \text { change }\end{array}$ & $7.3 \%$ & $2.9 \%$ & $2.9 \%$ & $0.0 \%$ \\
\hline
\end{tabular}

Graph 2 displays the skyrocketing expenditures for serials (in comparison to monograph expenditures). Note that Table 2 and Graph 2 have been revised since 2005-2006 and no longer include Serial Unit Cost or Serial Subscriptions. Beginning with 2006-2007, ARL libraries are reporting unduplicated counts of serial titles rather than subscriptions. 


\section{GRAPH 2}

\section{Median Monograph and Serial Expenditures in ARL Libraries, 1986-2008*}

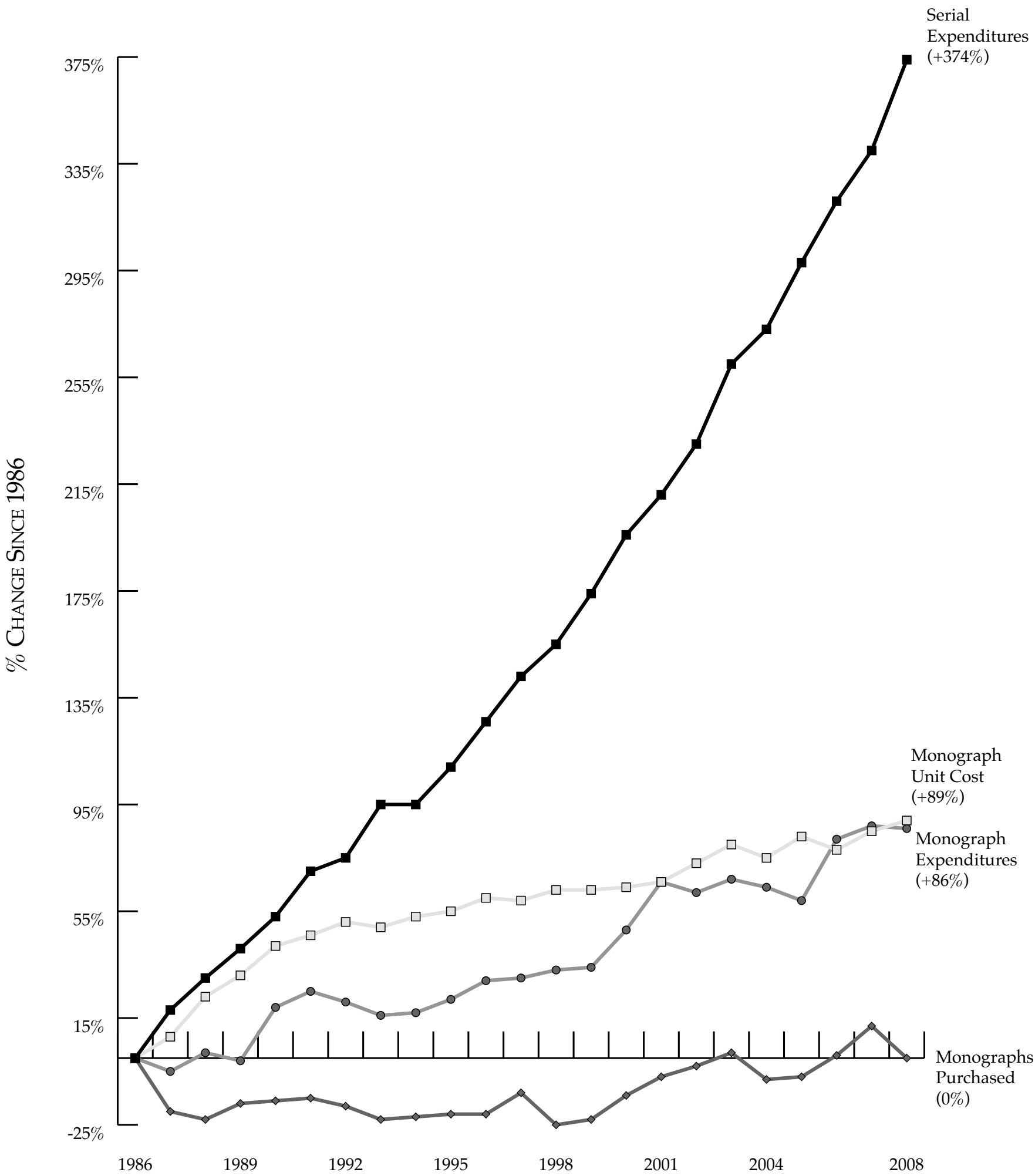

*Includes electronic resources from 1999-2000 onward.

Source: ARL Statistics 2007-2008, Association of Research Libraries, Washington, DC 


\section{TABLE 3}

\section{Supply AND Demand in ARL Libraries, 1986-2008 Median Values for Time-Series Trends}

\begin{tabular}{|c|c|c|c|c|c|c|}
\hline Year & $\begin{array}{r}\text { ILL: } \\
\text { Borrowed }\end{array}$ & $\begin{array}{l}\text { ILL: } \\
\text { Lent }\end{array}$ & $\begin{array}{l}\text { Graduate } \\
\text { Students }\end{array}$ & Faculty & $\begin{array}{r}\text { Total } \\
\text { Students }\end{array}$ & $\begin{array}{r}\text { Monographs } \\
\text { Purchased }\end{array}$ \\
\hline (Libraries) & (103) & (103) & (104) & (101) & (103) & (58) \\
\hline 1986 & 7,047 & 16,092 & 2,327 & 1,124 & 16,684 & 32,679 \\
\hline 1987 & 7,387 & 16,318 & 3,078 & 1,195 & 17,029 & 26,240 \\
\hline 1988 & 7,881 & 17,476 & 3,251 & 1,222 & 17,485 & 25,238 \\
\hline 1989 & 8,547 & 19,638 & 3,312 & 1,285 & 17,866 & 27,082 \\
\hline 1990 & 9,595 & 20,837 & 3,314 & 1,278 & 17,745 & 27,546 \\
\hline 1991 & 10,397 & 23,285 & 3,310 & 1,295 & 18,290 & 27,843 \\
\hline 1992 & 11,362 & 22,514 & 3,539 & 1,356 & 18,273 & 26,947 \\
\hline 1993 & 12,489 & 22,740 & 3,745 & 1,281 & 18,450 & 25,188 \\
\hline 1994 & 14,007 & 24,039 & 3,794 & 1,289 & 18,305 & 25,341 \\
\hline 1995 & 14,472 & 24,864 & 3,914 & 1,308 & 18,209 & 25,707 \\
\hline 1996 & 15,278 & 25,720 & 3,904 & 1,251 & 18,320 & 25,911 \\
\hline 1997 & 16,264 & 25,463 & 3,942 & 1,263 & 18,166 & 28,576 \\
\hline 1998 & 17,656 & 27,223 & 3,880 & 1,247 & 18,335 & 24,447 \\
\hline 1999 & 18,942 & 26,837 & 3,933 & 1,255 & 18,609 & 25,173 \\
\hline 2000 & 20,475 & 27,044 & 3,844 & 1,239 & 18,908 & 28,024 \\
\hline 2001 & 21,902 & 28,950 & 4,159 & 1,279 & 19,102 & 30,494 \\
\hline 2002 & 21,339 & 29,021 & 4,067 & 1,251 & 19,925 & 31,578 \\
\hline 2003 & 22,146 & 33,421 & 4,167 & 1,268 & 21,132 & 33,208 \\
\hline 2004 & 25,737 & 33,934 & 4,461 & 1,369 & 21,562 & 30,155 \\
\hline 2005 & 25,729 & 36,325 & 4,595 & 1,355 & 22,047 & 30,546 \\
\hline 2006 & 27,412 & 36,035 & 4,647 & 1,388 & 22,618 & 33,145 \\
\hline 2007 & 26,813 & 35,838 & 4,709 & 1,451 & 22,874 & 36,462 \\
\hline 2008 & 27,822 & 36,383 & 4,905 & 1,490 & 22,762 & 32,745 \\
\hline $\begin{array}{l}\text { Average annual } \\
\% \text { change }\end{array}$ & $6.4 \%$ & $3.8 \%$ & $3.4 \%$ & $1.3 \%$ & $1.4 \%$ & $0.0 \%$ \\
\hline
\end{tabular}

Interlibrary borrowing and lending, which showed a marked growth in the last decade, has continued to increase in 20072008. Monographs purchased in 2007-2008 are even with 1986 levels. 


\section{GRAPH 3}

\section{Supply ANd Demand in ARL Libraries, 1986-2008*}

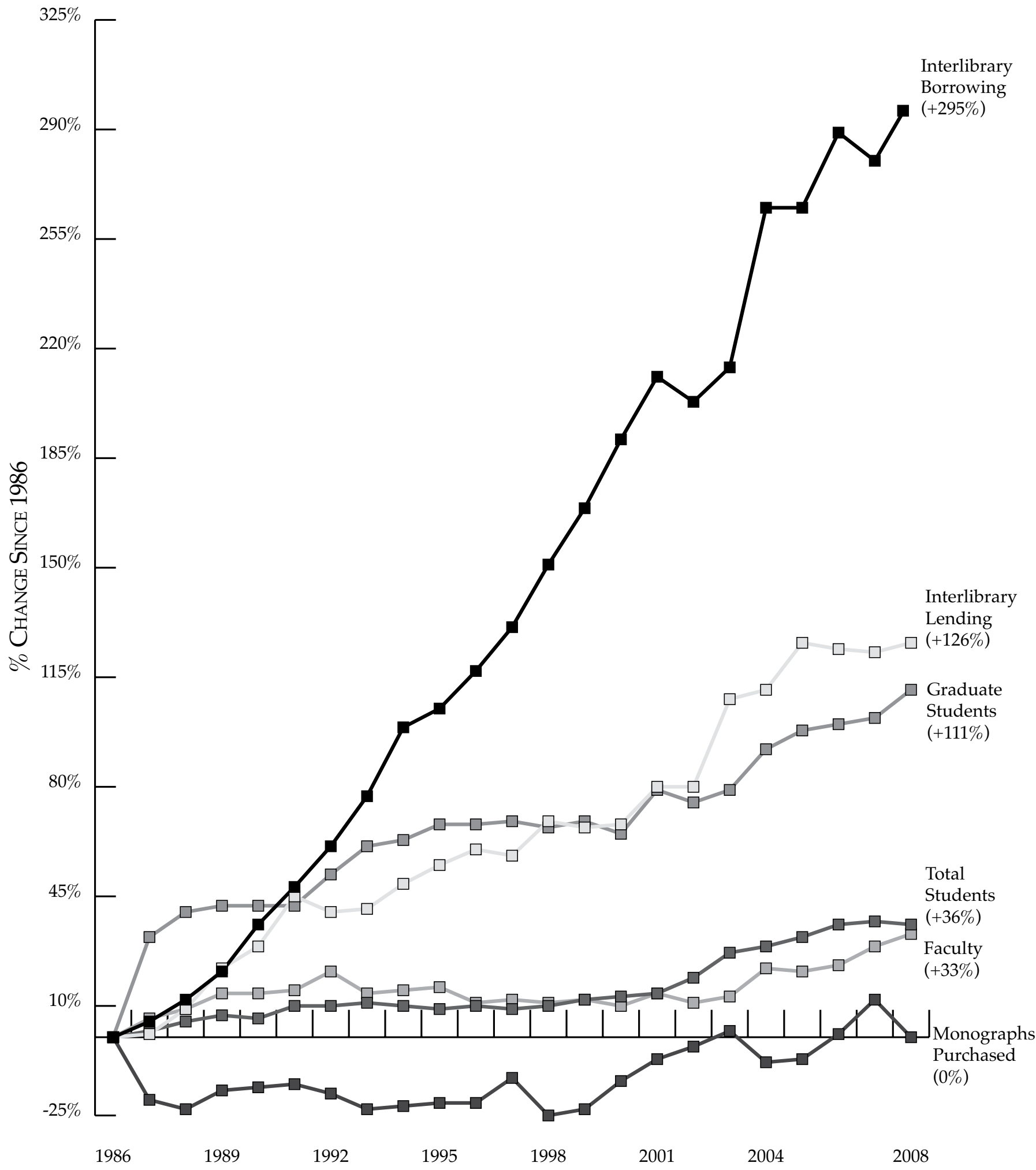

*Serial and monograph data includes electronic resources from 1999-2000 onward.

Source: ARL Statistics 2007-2008, Association of Research Libraries, Washington, DC 


\section{TABLE 4}

\section{EXPENDiture TRends in ARL LibRARIES, 1986-2008 Median Values for Time-Series Trends-Unadjusted dollar figures}

\begin{tabular}{|c|c|c|c|c|c|c|c|}
\hline Year & $\begin{array}{r}\text { Library } \\
\text { Materials }\end{array}$ & $\begin{array}{r}\text { Serial } \\
\text { Exp. }\end{array}$ & $\begin{array}{r}\text { Monograph } \\
\text { Exp. }\end{array}$ & $\begin{array}{r}\text { Total } \\
\text { Salary }\end{array}$ & $\begin{array}{r}\text { Operating } \\
\text { Exp. }\end{array}$ & Total & CPI \\
\hline (Libraries) & (105) & (100) & (97) & (104) & (104) & (105) & \\
\hline 1986 & $2,705,297$ & $1,496,775$ & $1,120,645$ & $4,011,436$ & 1,111,914 & $8,361,092$ & 109.6 \\
\hline 1987 & $3,058,479$ & $1,769,960$ & $1,064,484$ & $4,361,646$ & $1,180,167$ & $8,990,001$ & 113.6 \\
\hline 1988 & $3,369,896$ & $1,947,559$ & $1,141,226$ & $4,618,335$ & $1,198,674$ & $9,557,623$ & 118.3 \\
\hline 1989 & $3,577,405$ & $2,113,976$ & $1,106,073$ & $5,236,292$ & $1,364,558$ & $10,183,315$ & 124.0 \\
\hline 1990 & $3,903,358$ & $2,296,910$ & $1,330,747$ & $5,469,333$ & $1,386,618$ & $11,241,022$ & 130.7 \\
\hline 1991 & $4,064,344$ & $2,548,687$ & $1,400,738$ & $5,885,814$ & $1,445,735$ & $11,990,794$ & 136.2 \\
\hline 1992 & $4,156,510$ & $2,620,832$ & $1,353,865$ & $6,050,222$ & $1,390,245$ & $12,249,150$ & 140.3 \\
\hline 1993 & $4,316,674$ & $2,918,569$ & $1,295,807$ & $5,962,470$ & $1,561,122$ & $12,265,696$ & 144.5 \\
\hline 1994 & $4,572,276$ & $2,912,495$ & $1,309,807$ & $6,047,803$ & $1,676,701$ & $12,767,348$ & 148.2 \\
\hline 1995 & $4,715,203$ & $3,131,033$ & $1,365,575$ & $6,312,770$ & $1,853,586$ & $13,171,893$ & 152.4 \\
\hline 1996 & $5,126,482$ & $3,389,118$ & $1,444,015$ & $6,664,021$ & $1,997,233$ & $13,870,378$ & 156.9 \\
\hline 1997 & $5,562,742$ & $3,642,541$ & $1,460,234$ & $6,893,582$ & $2,039,957$ & $14,526,674$ & 160.5 \\
\hline 1998 & $5,795,223$ & $3,816,497$ & $1,486,764$ & $7,163,979$ & $2,072,903$ & $15,329,371$ & 163.0 \\
\hline 1999 & $6,232,365$ & $4,095,934$ & $1,506,651$ & $7,476,532$ & $2,069,887$ & 16,737,261 & 166.6 \\
\hline 2000 & $6,744,281$ & $4,430,812$ & $1,657,349$ & 7,811,403 & $1,991,852$ & $17,221,441$ & 172.2 \\
\hline 2001 & $7,322,507$ & $4,660,349$ & $1,864,023$ & $8,106,666$ & $2,280,493$ & $17,620,048$ & 177.5 \\
\hline 2002 & $7,599,249$ & $4,939,225$ & $1,812,826$ & $8,488,255$ & 2,136,616 & $18,456,038$ & 180.1 \\
\hline 2003 & $8,273,171$ & $5,392,007$ & 1,873,157 & $8,813,191$ & $2,073,913$ & 19,030,188 & 183.9 \\
\hline 2004 & $8,286,431$ & $5,580,157$ & $1,839,412$ & $9,015,741$ & $2,274,878$ & $19,953,776$ & 189.4 \\
\hline 2005 & $8,801,962$ & $5,962,446$ & 1,784,841 & $9,268,364$ & $2,243,592$ & $20,663,012$ & 195.4 \\
\hline 2006 & $9,174,734$ & $6,307,292$ & $2,044,272$ & $9,647,695$ & $2,540,171$ & $21,694,210$ & 203.5 \\
\hline 2007 & $9,600,793$ & 6,587,241 & 2,097,078 & $9,940,406$ & $2,549,139$ & $22,810,227$ & 208.3 \\
\hline 2008 & $10,454,644$ & $7,097,140$ & $2,084,991$ & $10,302,966$ & $2,649,899$ & $24,804,535$ & 219.9 \\
\hline $\begin{array}{l}\text { Average annual } \\
\% \text { change }\end{array}$ & $6.3 \%$ & $7.3 \%$ & $2.9 \%$ & $4.4 \%$ & $4.0 \%$ & $4.9 \%$ & $3.2 \%$ \\
\hline
\end{tabular}

Operating expenditures, including many automation expenditures, have doubled since 1986, but have remained at relatively stable levels of investment. The total salary expenditures median has increased only slightly over the past four years (average rate of 4\%). Monograph expenditures continue to increase, but in 2007-2008 have increased at a lower rate than the consumer price index (CPI). The annual consumer price index, included in Graph 4, provides a comparative reference for the increases in library expenditures. 


\section{GraPH 4}

\section{EXPENDiture Trends in ARL LibRaRies, 1986-2008}

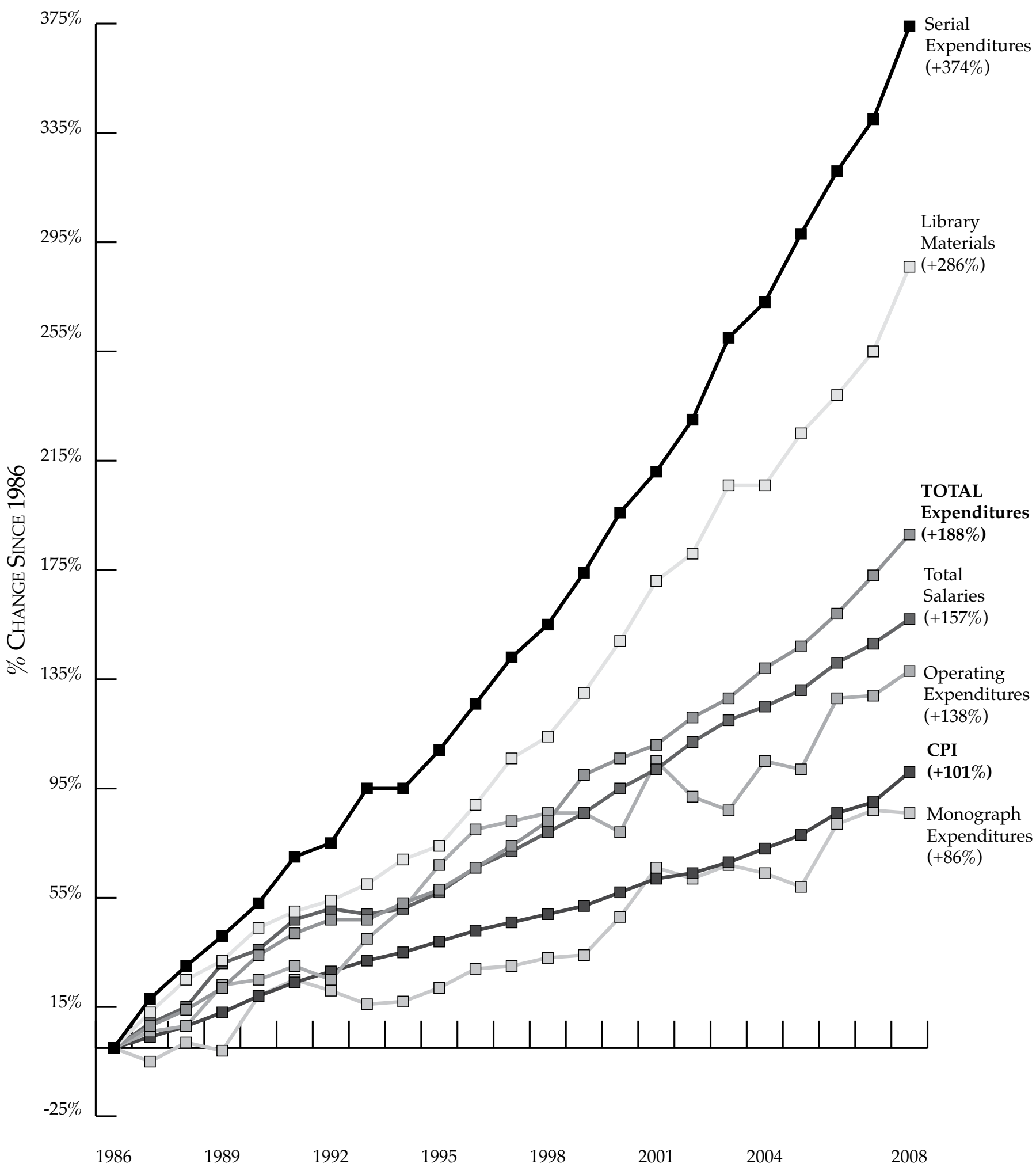

Source: ARL Statistics 2007-2008, Association of Research Libraries, Washington, DC 
TABLE 5

\section{Resources Per Student in ARL University Libraries, 1986-2008 Median of Ratio Values for Time-Series Trends}

\begin{tabular}{|c|c|c|c|c|c|}
\hline Year & $\begin{array}{r}\text { ILL: } \\
\text { Borrowed }\end{array}$ & $\begin{array}{r}\text { Volumes } \\
\text { Held }\end{array}$ & $\begin{array}{r}\text { Volumes } \\
\text { Added (Gross) }\end{array}$ & $\begin{array}{l}\text { Total } \\
\text { Staff }\end{array}$ & $\begin{array}{r}\text { Monographs } \\
\text { Purchased }\end{array}$ \\
\hline (Libraries) & (102) & (103) & (103) & (103) & (57) \\
\hline 1986 & 0.42 & 123.18 & 4.17 & 0.0159 & 2.20 \\
\hline 1987 & 0.44 & 125.31 & 3.92 & 0.0164 & 1.88 \\
\hline 1988 & 0.44 & 128.27 & 3.82 & 0.0161 & 1.66 \\
\hline 1989 & 0.50 & 130.04 & 3.84 & 0.0162 & 1.77 \\
\hline 1990 & 0.55 & 133.51 & 3.86 & 0.0155 & 1.83 \\
\hline 1991 & 0.63 & 137.11 & 4.04 & 0.0154 & 1.86 \\
\hline 1992 & 0.62 & 137.68 & 3.95 & 0.0153 & 1.58 \\
\hline 1993 & 0.68 & 146.02 & 3.76 & 0.0152 & 1.49 \\
\hline 1994 & 0.74 & 151.20 & 4.07 & 0.0152 & 1.55 \\
\hline 1995 & 0.80 & 154.00 & 4.37 & 0.0147 & 1.59 \\
\hline 1996 & 0.89 & 158.10 & 3.78 & 0.0145 & 1.48 \\
\hline 1997 & 0.89 & 159.40 & 4.03 & 0.0142 & 1.52 \\
\hline 1998 & 0.97 & 159.24 & 4.06 & 0.0143 & 1.44 \\
\hline 1999 & 1.05 & 159.37 & 4.00 & 0.0142 & 1.42 \\
\hline 2000 & 1.13 & 157.74 & 3.82 & 0.0139 & 1.63 \\
\hline 2001 & 1.16 & 155.71 & 3.89 & 0.0136 & 1.69 \\
\hline 2002 & 1.14 & 155.04 & 3.87 & 0.0131 & 1.54 \\
\hline 2003 & 1.14 & 156.41 & 3.52 & 0.0125 & 1.47 \\
\hline 2004 & 1.20 & 151.49 & 3.45 & 0.0120 & 1.53 \\
\hline 2005 & 1.22 & 157.93 & 3.30 & 0.0118 & 1.42 \\
\hline 2006 & 1.22 & 160.05 & 3.26 & 0.0117 & 1.64 \\
\hline 2007 & 1.13 & 159.49 & 3.49 & 0.0114 & 1.54 \\
\hline 2008 & 1.19 & 162.87 & 3.22 & 0.0110 & 1.40 \\
\hline $\begin{array}{l}\text { Average annual } \\
\% \text { change }\end{array}$ & $4.8 \%$ & $1.3 \%$ & $-1.2 \%$ & $-1.7 \%$ & $-2.0 \%$ \\
\hline
\end{tabular}

In 2007-2008, ARL libraries acquired 60\% fewer monographs per student than purchased in 1986. Libraries reported fewer staff per student in 2008 as compared to 1986: in 2008, there was a median number of 11 total staff per 1,000 students, compared to the 1986 figure of 16 per 1,000. 


\section{GraPH 5}

\section{Resources Per Student in ARL University Libraries, 1986-2008}

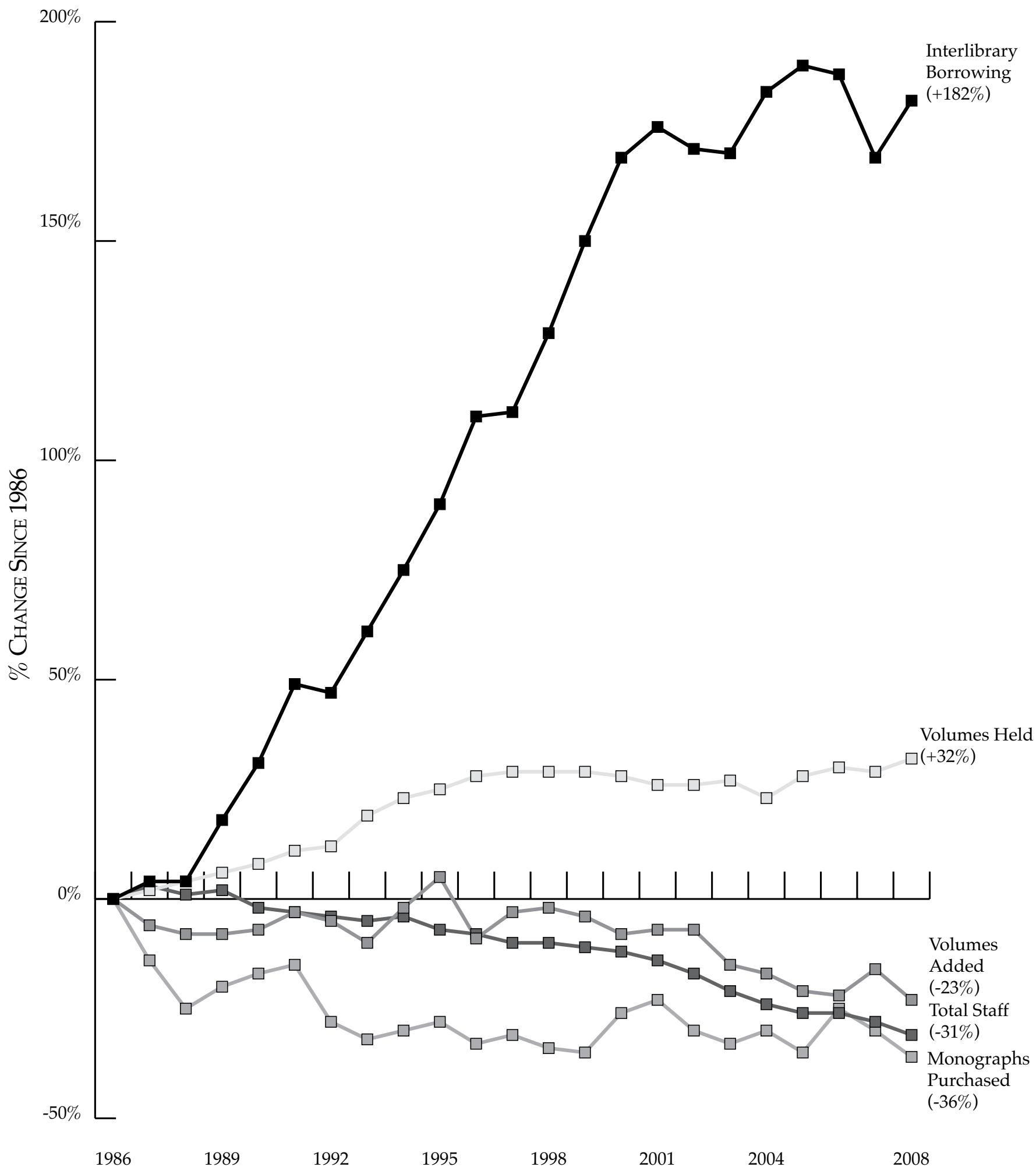

Source: ARL Statistics 2007-2008, Association of Research Libraries, Washington, DC 


\section{TABLE 6}

\section{Electronic Resources Expenditures in ARL University Libraries, 2007-2008}

\begin{tabular}{lcc}
\hline & Sum & Number Reporting \\
\hline $\begin{array}{l}\text { Expenditures for Computer Files } \\
\text { (one-time/monographic purchase) }\end{array}$ & $\$ 73,102,024$ & 108 \\
$\begin{array}{l}\text { Expenditures for Electronic Serials } \\
\text { Expenditures for Bibliographic Utilities, Networks, etc. } \\
\text { (Library) }\end{array}$ & $\$ 554,880,484$ & 110 \\
$\begin{array}{l}\text { Expenditures for Bibliographic Utilities, Networks, etc. } \\
\text { (External) }\end{array}$ & $\$ 21,079,241$ & \\
& & \\
Expenditures for Hardware and Software & & 103 \\
Expenditures for Document Delivery/Interlibrary Loan & $\$ 14,027,391$ & 108 \\
\hline
\end{tabular}

Table 6 contains data on several items which previously had been collected only in the ARL Supplementary Statistics. These data are especially useful because they reflect monies spent on all electronic serials, while the ARL Statistics categories of "serials purchased" and "serials expenditures" include only those journals that provide full-text electronic versions to their subscribers. The Expenditures for Electronic Serials time series may be viewed as an alternative to the Serials Purchased series, both figures reflecting in their own way the influence the electronic serial is gaining in the modern research library.

Not only have electronic materials expenditures grown sharply in the past decade, they have grown at a rate far exceeding that of library materials expenditures overall. As shown in Graph 6, in every year of the last decade electronic materials expenditures have grown sharply, anywhere between two and ten times faster than other materials expenditures have.

The average ARL university library now spends $51 \%$ of its materials budget on electronic resources (Tables 7A and 7B) and 68 ARL libraries report that they spent more than $50 \%$ of their materials budget on electronic resources (see Rank Order Table 20). 


\section{GraPh 6}

\section{Electronic Resources vs. Total Materials Expenditures, 1993-2008 \\ Yearly InCReases in Average Expenditures}

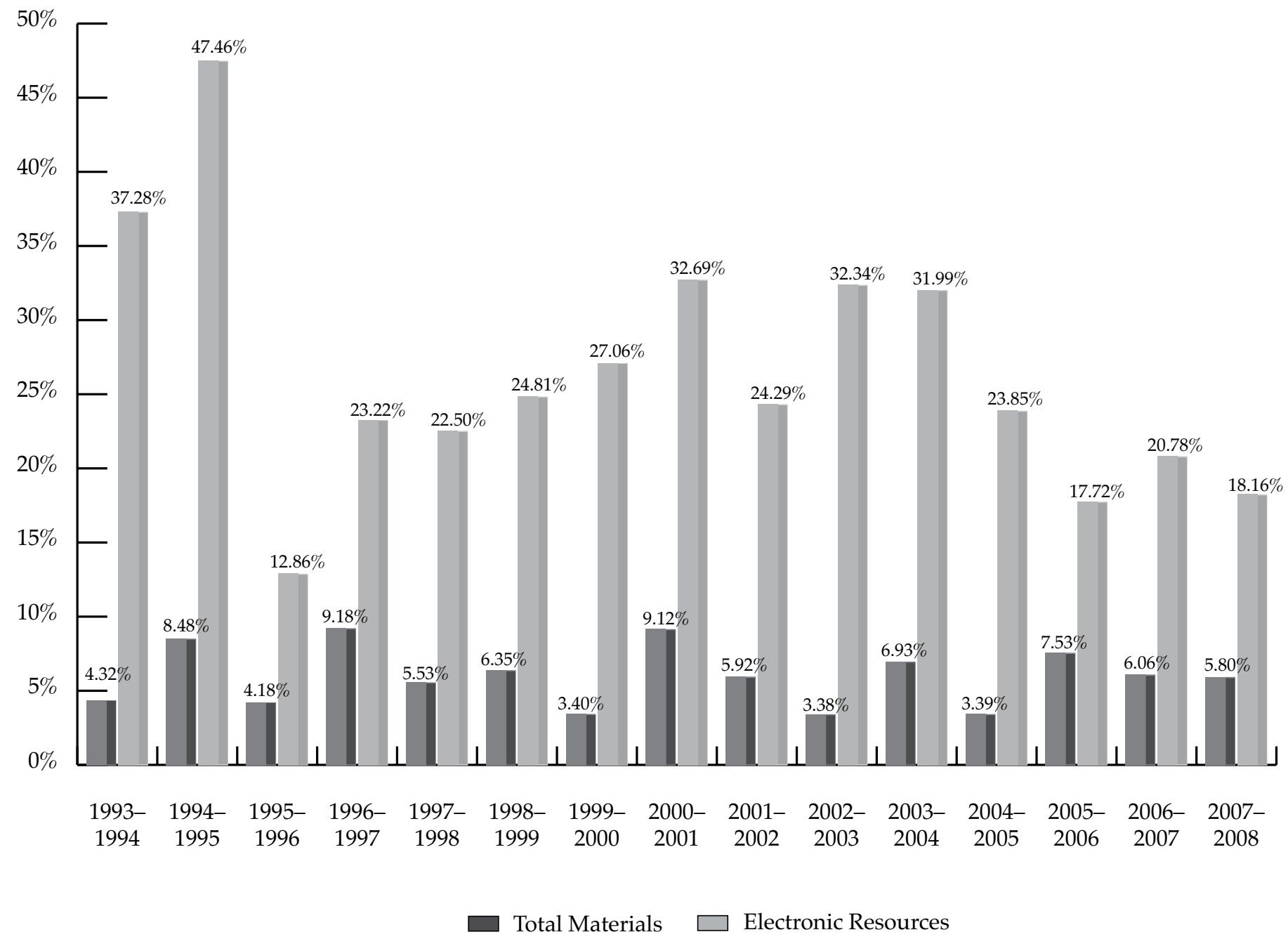




\section{TABLE 7A}

\section{Electronic Resources and Materials Expenditures in ARL University Libraries, 1992-2002}

\begin{tabular}{|c|c|c|c|c|c|c|c|c|c|c|}
\hline & 1992-1993 & 1993-1994 & 1994-1995 & 1995-1996 & 1996-1997 & 1997-1998 & 1998-1999 & 1999-2000 & $2000-2001$ & 2001-2002 \\
\hline \multicolumn{11}{|c|}{ a. Computer File Expenditures (monographic/onetime) } \\
\hline Total & $14,147,625$ & $20,132,553$ & $22,030,727$ & $24,639,822$ & $8,013,055$ & $11,189,103$ & $10,848,219$ & $14,727,984$ & $15,297,096$ & $16,748,194$ \\
\hline Average & 172,532 & 236,854 & 247,536 & 262,126 & 87,098 & 122,957 & 121,890 & 161,846 & 159,345 & 167,482 \\
\hline Median & 148,158 & 212,936 & 217,988 & 219,178 & 47,932 & 52,311 & 54,024 & 98,657 & 72,070 & 82,566 \\
\hline $\mathbf{N}$ & & 85 & 89 & 94 & 92 & 91 & 89 & 91 & 96 & 100 \\
\hline \multicolumn{11}{|c|}{ b. Electronic Serial Expenditures } \\
\hline Total & N/A & N/A & $11,847,577$ & $15,170,971$ & $40,956,696$ & $49,497,141$ & $67,124,554$ & $84,343,868$ & $117,415,618$ & $154,418,679$ \\
\hline Average & $\mathrm{N} / \mathrm{A}$ & $\mathrm{N} / \mathrm{A}$ & 188,057 & 194,500 & 401,536 & 494,971 & 639,281 & 818,873 & $1,118,244$ & $1,429,803$ \\
\hline Median & $\mathrm{N} / \mathrm{A}$ & $\mathrm{N} / \mathrm{A}$ & 156,754 & 172,805 & 355,922 & 426,722 & 571,790 & 736,317 & 992,067 & $1,272,965$ \\
\hline $\mathbf{N}$ & & & 63 & 78 & 102 & 100 & 105 & 103 & 105 & 108 \\
\hline \multicolumn{11}{|c|}{ c. Total Electronic Resources (Total $a+b)$} \\
\hline Total & $14,147,625$ & $20,132,553$ & $33,878,304$ & $39,810,793$ & $50,512,984$ & $60,686,244$ & $77,972,773$ & $99,071,852$ & $132,712,714$ & $171,166,873$ \\
\hline Average & 172,532 & 236,854 & 349,261 & 394,166 & 485,702 & 594,963 & 742,598 & 943,541 & $1,252,007$ & $1,556,062$ \\
\hline Median & 148,158 & 212,936 & 278,404 & 332,128 & 420,741 & 495,011 & 645,495 & 931,210 & $1,129,298$ & $1,377,874$ \\
\hline $\mathbf{N}$ & 82 & 85 & 97 & 101 & 104 & 102 & 105 & 105 & 106 & 110 \\
\hline \multicolumn{11}{|c|}{ Total Library Materials Expenditures* } \\
\hline Total & $393,271,073$ & $425,287,651$ & $526,496,347$ & $571,145,986$ & $642,123,715$ & $664,600,663$ & $727,623,160$ & $773,321,519$ & $828,778,808$ & $910,930,849$ \\
\hline Average & $4,795,989$ & $5,003,384$ & $5,427,797$ & $5,654,911$ & $6,174,266$ & $6,515,692$ & $6,929,744$ & $7,364,967$ & $7,818,668$ & $8,281,189$ \\
\hline Median & $4,242,887$ & $4,527,122$ & $4,714,384$ & $4,975,353$ & $5,529,606$ & $5,643,070$ & $5,991,177$ & $6,545,146$ & $7,028,134$ & $7,566,727$ \\
\hline $\mathbf{N}$ & 82 & 85 & 97 & 101 & 104 & 102 & 105 & 105 & 106 & 110 \\
\hline \multicolumn{11}{|c|}{ Electronic Resources Expenditures as a Percent of Total Materials Expenditures } \\
\hline Average & 3.60 & 4.75 & 6.39 & 6.83 & 7.76 & 8.85 & 10.56 & 12.88 & 16.25 & 19.60 \\
\hline Median & N/A & 4.45 & 5.33 & 6.42 & 7.51 & 8.29 & 10.18 & 12.75 & 14.80 & 18.15 \\
\hline $\mathbf{N}$ & 82 & 85 & 97 & 101 & 104 & 102 & 105 & 105 & 106 & 110 \\
\hline \multicolumn{11}{|c|}{ Expenditures for Bibliographic Utilities, Networks, etc. (External) } \\
\hline Total & N/A & N/A & N/A & N/A & $\$ 3,827,348$ & $4,695,737$ & $7,442,962$ & $9,523,348$ & $14,655,078$ & $20,373,560$ \\
\hline Average & N/A & N/A & N/A & N/A & $\$ 136,691$ & 142,295 & 201,161 & 250,614 & 311,810 & 424,449 \\
\hline Median & N/A & N/A & N/A & N/A & $\$ 120,096$ & 128,795 & 145,280 & 204,598 & 198,289 & 336,690 \\
\hline $\mathbf{N}$ & & & & & 28 & 33 & 37 & 38 & 47 & 48 \\
\hline
\end{tabular}

*Figures reflect Materials Expenditures only from those institutions that reported nonzero figures for Total Electronic Resources. 


\section{TABLE 7B}

\section{Electronic Resources and Materials Expenditures in ARL University Libraries, 2002-2008}

\begin{tabular}{|c|c|c|c|c|c|c|}
\hline & $2002-2003$ & $2003-2004$ & $2004-2005$ & $2005-2006$ & $2006-2007$ & 2007-2008 \\
\hline \multicolumn{7}{|c|}{ a. Computer File Expenditures (monographic/onetime) } \\
\hline Total & $\$ 23,275,683$ & $\$ 32,098,404$ & $\$ 38,744,076$ & $\$ 48,793,981$ & $\$ 59,808,658$ & $\$ 73,102,024$ \\
\hline Average & $\$ 225,978$ & $\$ 314,690$ & $\$ 372,539$ & $\$ 478,372$ & $\$ 558,959$ & $\$ 676,574$ \\
\hline Median & $\$ 111,266$ & $\$ 191,148$ & $\$ 210,576$ & $\$ 336,338$ & $\$ 352,802$ & $\$ 410,202$ \\
\hline $\mathbf{N}$ & 103 & 102 & 104 & 102 & 107 & 108 \\
\hline \multicolumn{7}{|c|}{ b. Electronic Serial Expenditures } \\
\hline Total & $\$ 205,300,292$ & $\$ 269,601,241$ & $\$ 328,166,027$ & $\$ 383,127,163$ & $\$ 476,225,086$ & $\$ 554,637,844$ \\
\hline Average & $\$ 1,849,552$ & $\$ 2,450,920$ & $\$ 3,038,574$ & $\$ 3,547,474$ & $\$ 4,290,316$ & $\$ 5,042,162$ \\
\hline Median & $\$ 1,649,361$ & $\$ 2,348,463$ & $\$ 2,824,962$ & $\$ 3,349,709$ & $\$ 4,240,530$ & $\$ 4,899,366$ \\
\hline $\mathbf{N}$ & 111 & 110 & 108 & 108 & 111 & 110 \\
\hline \multicolumn{7}{|c|}{ c. Total Electronic Resources (Total $a+b)$} \\
\hline Total & $\$ 228,575,974$ & $\$ 301,699,645$ & $\$ 366,910,103$ & $\$ 431,921,144$ & $\$ 536,033,744$ & $\$ 627,707,869$ \\
\hline Average & $\$ 2,059,243$ & $\$ 2,718,015$ & $\$ 3,366,147$ & $\$ 3,962,579$ & $\$ 4,786,016$ & $\$ 5,655,026$ \\
\hline Median & $\$ 1,775,865$ & $\$ 2,705,847$ & $\$ 3,144,841$ & $\$ 3,792,873$ & $\$ 4,661,123$ & $\$ 5,410,421$ \\
\hline $\mathbf{N}$ & 111 & 111 & 109 & 109 & 112 & 111 \\
\hline \multicolumn{7}{|c|}{ Total Library Materials Expenditures* } \\
\hline Total & $\$ 950,275,167$ & $\$ 1,016,121,605$ & $\$ 1,031,619,722$ & $\$ 1,109,340,878$ & $\$ 1,219,796,179$ & $\$ 1,290,575,224$ \\
\hline Average & $\$ 8,561,038$ & $\$ 9,154,249$ & $\$ 9,464,401$ & $\$ 10,177,439$ & $\$ 10,794,656$ & $\$ 11,421,020$ \\
\hline Median & $\$ 7,707,153$ & $\$ 8,276,175$ & $\$ 8,662,668$ & $\$ 9,156,974$ & $\$ 9,594,560$ & $\$ 10,388,129$ \\
\hline $\mathbf{N}$ & 111 & 111 & 109 & 109 & 113 & 113 \\
\hline \multicolumn{7}{|c|}{ Electronic Resources Expenditures as a Percent of Total Materials Expenditures } \\
\hline Average & 25.02 & 31.33 & 37.46 & 40.93 & 46.55 & 51.46 \\
\hline Median & 22.01 & 29.81 & 37.53 & 43.14 & 47.68 & 53.06 \\
\hline $\mathbf{N}$ & 111 & 111 & 109 & 109 & 112 & 111 \\
\hline \multicolumn{7}{|c|}{ Expenditures for Bibliographic Utilities, Networks, etc. (External) } \\
\hline Total & $\$ 21,470,716$ & $\$ 17,420,520$ & $\$ 16,082,790$ & $\$ 15,930,476$ & $\$ 18,931,797$ & $\$ 21,079,241$ \\
\hline Average & $\$ 438,178$ & $\$ 335,010$ & $\$ 349,626$ & $\$ 318,610$ & $\$ 225,379$ & $\$ 242,290$ \\
\hline Median & $\$ 250,000$ & $\$ 94,837$ & $\$ 149,396$ & $\$ 143,649$ & $\$ 33,247$ & $\$ 54,750$ \\
\hline $\mathbf{N}$ & 49 & 52 & 46 & 50 & 84 & 87 \\
\hline
\end{tabular}

\footnotetext{
* Figures reflect Materials Expenditures only from those institutions that reported nonzero figures for Total Electronic Resources.
} 
The ARL Statistics are available through an interactive edition on the Web, supplementing previously available data from 1992-1993. Maintained and supported by the Geospatial and Statistical Data Center at the University of Virginia, this site offers researchers access to ARL Statistics with documentation. ${ }^{1}$

Note that currently the site is aging and tables have not been updated with the latest data. ARL Index data are erroneous. Updated index data may be found at http://www.arl.org/stats/index/index.shtml. We do have plans to migrate the site to a new platform at ARL during 2009-2010.

The site offers maps and graphics depicting library trends. The interactive pages also include options for regional aggregates, customized samples of observations, simple rank order tabulations of data, and the ability to create ratios of any two variables. These and other capabilities are located at http://fisher.lib.virginia.edu/arl/.

The ARL Statistics datafiles since 1908 can be retrieved in spreadsheet format from http://www.arl.org/stats/ annualsurveys/arlstats/mrstat.shtml.

The ARL Statistics and Measurement Program Web site is located at http://www.arl.org/stats/.

$\overline{1}$ For more information on the ARL Statistics Interactive Edition, see Martha Kyrillidou, "To Describe and Measure the Performance of North American Research Libraries," IFLA Journal 27 (2001): 257-63. 
ARL Library Data TABles

2007-2008 


\section{Collections: Volumes and MonographS}

Volumes In Library Volumes Added (Gross)

(1)

(1b.i) (Net)

(1b)
Monographs Purchased (Volumes)

(Survey Question Number)

(2)

INSTITUTION

Notes

\begin{tabular}{|c|c|c|c|c|c|}
\hline ALABAMA & bGLM+ & $2,892,166$ & 53,470 & 47,779 & 32,646 \\
\hline ALBERTA & G+ & $6,890,679$ & 186,313 & 169,904 & UA/NA \\
\hline ARIZONA & bGLM+ & $5,722,280$ & 69,004 & 19,544 & 37,870 \\
\hline ARIZONA STATE & BGL+ & $4,422,239$ & 84,467 & 70,744 & 52,392 \\
\hline AUBURN & bG+ & $3,053,468$ & 38,068 & 36,482 & 12,224 \\
\hline BOSTON & LM+ & $2,783,137$ & 35,581 & 28,137 & 15,001 \\
\hline BOSTON COLLEGE & $\mathrm{L}+$ & $2,512,605$ & 40,325 & 25,464 & 29,728 \\
\hline BRIGHAM YOUNG & $\mathrm{L}+$ & $4,080,079$ & 187,005 & 171,952 & 153,553 \\
\hline BRITISH COLUMBIA & G+ & $6,040,261$ & 122,887 & 110,664 & $\mathrm{UA} / \mathrm{NA}$ \\
\hline BROWN & bG+ & $3,825,413$ & 87,499 & 82,463 & 49,939 \\
\hline CALIFORNIA BERKELEY & GL+ & $11,087,687$ & 207,779 & 189,921 & UA/NA \\
\hline CALIFORNIA DAVIS & GLM+ & $3,683,867$ & 91,692 & 81,761 & UA/NA \\
\hline CALIFORNIA IRVINE & bGM+ & $3,049,706$ & 79,732 & 76,298 & 35,890 \\
\hline CALIFORNIA LOS ANGELES & LM+ & $8,393,910$ & 121,136 & 111,385 & 56,557 \\
\hline CALIFORNIA RIVERSIDE & G+ & $2,527,607$ & 49,797 & 44,405 & 25,318 \\
\hline CALIFORNIA SAN DIEGO & bGM+ & $3,372,785$ & 108,073 & 12,343 & 42,823 \\
\hline CALIFORNIA SANTA BARBARA & G+ & $2,948,999$ & 40,432 & 34,460 & 28,273 \\
\hline CASE WESTERN RESERVE & GLM+ & $2,751,662$ & 26,072 & 16,203 & 10,339 \\
\hline CHICAGO & bGLM+ & $8,597,159$ & 277,507 & 245,296 & 125,542 \\
\hline CINCINNATI & bGLM+ & $3,631,658$ & 95,810 & 83,771 & 33,903 \\
\hline COLORADO & bGL+ & $3,928,431$ & 98,800 & 84,973 & 33,104 \\
\hline COLORADO STATE & G+ & $2,045,603$ & 53,932 & 51,347 & 26,797 \\
\hline COLUMBIA & GLM+ & $10,296,816$ & 547,346 & 291,968 & 468,515 \\
\hline CONNECTICUT & BGLM+ & $3,919,423$ & 34,095 & $-45,478$ & 19,975 \\
\hline CORNELL & BGL+ & $8,141,781$ & 126,058 & 111,015 & UA/NA \\
\hline DARTMOUTH & $\mathrm{GM}+$ & $2,785,925$ & 58,147 & 53,097 & 29,420 \\
\hline DELAWARE & G+ & $2,779,271$ & 39,898 & 38,056 & 27,944 \\
\hline DUKE & bLM+ & $5,950,442$ & 105,419 & 100,834 & 67,879 \\
\hline EMORY & BGLM+ & $3,377,140$ & 89,560 & 80,386 & 50,483 \\
\hline FLORIDA & BbGLM+ & $4,288,118$ & 65,257 & 57,415 & 41,448 \\
\hline FLORIDA STATE & BbLM+ & $2,844,624$ & 55,821 & 41,133 & 45,911 \\
\hline GEORGE WASHINGTON & GLM+ & $2,238,894$ & 54,595 & 19,045 & 24,738 \\
\hline GEORGETOWN & bGLM+ & $3,041,624$ & 98,442 & 86,346 & 41,525 \\
\hline GEORGIA & GL+ & $4,637,291$ & 83,468 & 78,071 & 48,717 \\
\hline GEORGIA TECH & G+ & $2,524,671$ & 41,514 & 37,117 & 7,926 \\
\hline GUELPH & BG+ & $1,853,628$ & 23,025 & 6,830 & 17,399 \\
\hline HARVARD & GLM+ & $16,250,117$ & 319,547 & 304,407 & UA/NA \\
\hline HAWAII & GLM+ & $3,533,671$ & 52,882 & 46,734 & 21,005 \\
\hline HOUSTON & bL+ & $2,617,282$ & 66,505 & 59,213 & 25,062 \\
\hline
\end{tabular}




\section{Collections: Volumes and MonographS}

Volumes In Library Volumes Added (Gross)

(1)

(Survey Question Number)
Volumes Added Monographs Purchased (Net) (Volumes)

(1b)

(2)

\begin{tabular}{|c|c|c|c|c|c|}
\hline INSTITUTION & Notes & & & & \\
\hline HOWARD & bLM+ & $2,455,985$ & 24,428 & 20,736 & 4,496 \\
\hline ILLINOIS CHICAGO & $\mathrm{M}+$ & $2,447,459$ & 97,904 & 83,754 & 39,349 \\
\hline ILLINOIS URBANA & bGL+ & $11,686,060$ & 208,659 & 203,495 & 72,722 \\
\hline INDIANA & GLM+ & $8,723,253$ & 174,417 & 170,447 & 85,681 \\
\hline IOWA & bLM+ & $4,949,636$ & 185,900 & 158,306 & 61,470 \\
\hline IOWA STATE & G+ & $2,529,921$ & 43,326 & 29,041 & 28,436 \\
\hline JOHNS HOPKINS & $\mathrm{BbG}+$ & $3,740,029$ & 37,223 & 15,435 & 26,671 \\
\hline KANSAS & $\mathrm{BbLM}+$ & $4,235,542$ & 56,777 & 43,706 & 51,625 \\
\hline KENT STATE & $\mathrm{BbG}+$ & $2,853,965$ & 55,781 & 44,486 & 14,724 \\
\hline KENTUCKY & GLM+ & $3,719,548$ & 85,805 & 83,357 & 42,529 \\
\hline LAVAL & G+ & $2,894,709$ & 73,125 & 60,590 & 40,357 \\
\hline LOUISIANA STATE & GLM+ & $4,082,803$ & 37,946 & 15,634 & 33,655 \\
\hline LOUISVILLE & $\mathrm{BbG}+$ & 2,191,091 & 68,955 & 47,809 & 27,300 \\
\hline MCGILL & $\mathrm{BbGLM+}$ & $4,498,389$ & 89,015 & 37,349 & 378,866 \\
\hline MCMASTER & GM+ & $2,280,435$ & 178,927 & 114,854 & 32,843 \\
\hline MANITOBA & G+ & $2,284,284$ & 67,745 & 48,340 & 50,404 \\
\hline MARYLAND & G & $3,716,860$ & 105,517 & 93,484 & 36,951 \\
\hline MASSACHUSETTS & G+ & $3,578,753$ & 52,701 & 49,334 & 18,771 \\
\hline MIT & G+ & $2,914,052$ & 75,469 & 66,372 & 23,268 \\
\hline MIAMI & bGLM+ & $3,265,503$ & 76,116 & 37,560 & 45,768 \\
\hline MICHIGAN & GLM+ & $9,175,102$ & 146,729 & 99,477 & 99,784 \\
\hline MICHIGAN STATE & GL+ & $4,994,033$ & 85,666 & 78,412 & 52,719 \\
\hline MINNESOTA & bGLM+ & $6,877,699$ & 120,523 & 99,698 & 43,115 \\
\hline MISSOURI & GL+ & $3,494,470$ & 45,462 & 39,885 & 26,196 \\
\hline MONTREAL & GLM+ & $3,183,053$ & 62,208 & 32,667 & 50,515 \\
\hline NEBRASKA & GL+ & $3,204,060$ & 49,588 & 36,351 & 8,302 \\
\hline NEW MEXICO & GLM+ & $2,737,747$ & 51,768 & 29,917 & 20,116 \\
\hline NEW YORK & bGLM+ & $5,073,286$ & 107,390 & 85,022 & 55,503 \\
\hline NORTH CAROLINA & GLM+ & $6,526,824$ & 126,279 & 114,719 & 73,359 \\
\hline NORTH CAROLINA STATE & G+ & $3,981,278$ & 126,853 & 123,913 & 29,085 \\
\hline NORTHWESTERN & BGLM+ & $4,842,949$ & 79,608 & 74,361 & 38,867 \\
\hline NOTRE DAME & bGL+ & $3,393,290$ & 75,659 & 70,895 & 49,727 \\
\hline $\mathrm{OHIO}$ & $\mathrm{BbG}+$ & $2,893,238$ & 67,904 & 51,349 & 27,809 \\
\hline OHIO STATE & bGLM+ & $6,285,446$ & 161,118 & 41,351 & 67,611 \\
\hline OKLAHOMA & GLM+ & $5,299,924$ & 257,137 & 252,278 & 30,327 \\
\hline OKLAHOMA STATE & BGM+ & $2,867,680$ & 71,378 & 69,608 & 22,598 \\
\hline OREGON & bGL+ & $2,959,739$ & 58,993 & 54,231 & 22,450 \\
\hline PENNSYLVANIA & GLM+ & $6,096,592$ & 113,609 & 103,510 & UA/NA \\
\hline PENNSYLVANIA STATE & BGLM+ & $5,354,645$ & 184,457 & 132,972 & 59,330 \\
\hline
\end{tabular}




\section{Collections: Volumes and MonographS}

Volumes In Library Volumes Added (Gross)

(1)

(1b.i)
Volumes Added (Net)

(1b)
Monographs Purchased (Volumes)

(Survey Question Number)

(2)

INSTITUTION

PITTSBURGH

PRINCETON

PURDUE

QUEENS

RICE

ROCHESTER

RUTGERS

SASKATCHEWAN

SOUTH CAROLINA

SOUTHERN CALIFORNIA

SOUTHERN ILLINOIS

SUNY-ALBANY

SUNY-BUFFALO

SUNY-STONY BROOK

SYRACUSE

TEMPLE

TENNESSEE

TEXAS

TEXAS A\&M

TEXAS TECH

TORONTO

TULANE

UTAH

VANDERBILT

VIRGINIA

VIRGINIA TECH

WASHINGTON

WASHINGTON STATE

WASHINGTON U.-ST. LOUIS

WATERLOO

WAYNE STATE

WESTERN ONTARIO

WISCONSIN

YALE

YORK

BOSTON PUBLIC

CISTI

CENTER FOR RESEARCH LIBRARIES

LIBRARY OF CONGRESS

Notes

\begin{tabular}{|c|c|c|c|c|}
\hline BGLM+ & $5,657,403$ & 160,722 & 151,484 & $\mathrm{UA} / \mathrm{NA}$ \\
\hline bG+ & $6,941,254$ & 174,320 & 162,579 & 103,438 \\
\hline G+ & $2,509,158$ & 43,874 & 4,355 & 18,324 \\
\hline $\mathrm{LM}+$ & $2,542,264$ & 42,383 & 24,936 & 27,734 \\
\hline G+ & $2,570,768$ & 52,881 & 52,109 & 42,064 \\
\hline $\mathrm{bM}+$ & $3,701,241$ & 38,465 & 27,076 & 21,476 \\
\hline BGL+ & $4,529,396$ & 81,114 & 67,147 & 21,183 \\
\hline GLM+ & $2,029,016$ & 31,988 & 12,307 & 20,877 \\
\hline $\mathrm{LM}+$ & $3,629,954$ & 50,393 & 47,992 & 18,830 \\
\hline BGLM+ & $4,084,284$ & 58,810 & 45,056 & 53,374 \\
\hline $\mathrm{LM}+$ & $3,099,151$ & 35,178 & 25,486 & 17,299 \\
\hline G+ & $2,225,241$ & 92,459 & 75,076 & 16,845 \\
\hline GLM+ & $3,720,113$ & 82,074 & 65,024 & 25,253 \\
\hline $\mathrm{M}+$ & $2,257,590$ & 25,594 & 16,828 & 8,061 \\
\hline GL+ & $3,174,700$ & 30,585 & 14,460 & 20,601 \\
\hline BbGLM+ & $3,205,539$ & 68,903 & 67,923 & 47,318 \\
\hline BbGLM+ & $3,289,447$ & 67,867 & 36,684 & 37,010 \\
\hline GL+ & $9,447,434$ & 178,892 & 124,182 & 48,857 \\
\hline $\mathrm{BbG}+$ & $3,933,535$ & 206,223 & 194,454 & 64,803 \\
\hline BLM+ & $2,617,844$ & 54,715 & 39,659 & 53,560 \\
\hline $\mathrm{B}+$ & $11,186,488$ & 247,666 & 232,861 & 127,855 \\
\hline BLM+ & $3,257,609$ & 113,591 & 110,656 & 43,740 \\
\hline LM & $3,373,141$ & 55,501 & 52,974 & UA/NA \\
\hline LM+ & $3,440,317$ & 59,525 & 46,798 & 28,145 \\
\hline GLM+ & $5,532,266$ & 91,904 & 67,189 & 56,260 \\
\hline G & $2,331,055$ & 33,780 & 31,629 & 19,391 \\
\hline GLM+ & $7,409,221$ & 139,683 & 104,865 & 51,043 \\
\hline $\mathrm{BG}+$ & $2,326,652$ & 35,166 & 31,715 & 15,383 \\
\hline bGLM+ & $4,224,113$ & 74,408 & 61,133 & 26,689 \\
\hline $\mathrm{G}^{+}$ & $2,286,415$ & 30,818 & 26,933 & 57,664 \\
\hline+ & $3,660,642$ & 39,045 & 2,003 & 23,615 \\
\hline GL+ & $3,546,496$ & 50,007 & 48,807 & 27,642 \\
\hline GLM+ & $8,059,335$ & 139,835 & 125,699 & 55,530 \\
\hline $\mathrm{LM}+$ & $12,519,514$ & 238,862 & 235,920 & 167,433 \\
\hline BGL+ & $3,151,882$ & 53,699 & 49,858 & 34,848 \\
\hline G & $7,454,800$ & 8,335 & $-177,190$ & 133,998 \\
\hline+ & $8,176,131$ & 16,748 & 14,913 & 13,108 \\
\hline+ & $2,228,576$ & 10,286 & 10,286 & 4,600 \\
\hline bG+ & $30,207,310$ & 438,027 & 432,132 & 139,936 \\
\hline
\end{tabular}




\section{Collections: Volumes And Monographs}

Volumes In Library $\quad$ Volumes Added

(Gross)
Volumes Added

(Net)
Monographs Purchased

(Volumes)

(Survey Question Number)

(1)

(1b.i)

(1b)

(2)

INSTITUTION

Notes

\begin{tabular}{llrrr}
\hline NATIONAL AGRICULTURAL LIBRARY & bG+ & $2,442,583$ & 12,153 & 8,631 \\
LIBRARY \& ARCHIVES CANADA & G + & UA/NA & UA/NA & UA/NA \\
NATIONAL LIBRARY OF MEDICINE & G+ & $2,594,276$ & 37,953 & 35,303 \\
NEW YORK PUBLIC & + & $9,125,939$ & 169,530 & 169,522 \\
NEW YORK STATE & G+ & $2,711,029$ & 22,345 & 22,340 \\
SMITHSONIAN & $\mathrm{G}+$ & $1,354,810$ & 125,221 & 124,034 \\
& & & 94,614
\end{tabular}

+ - See Footnotes

L - Includes Law library

M- Includes Medical library
G - Government documents not included in serials count B - Includes branch campuses

$\mathrm{b}-$ Basis of volume count is bibliographic
U/A - Unavailable

N/A - Not Applicable

. - no data supplied

\section{Summary Data}

Volumes in Library Volumes Added

(Gross)

Volumes Added

(Net)

Monographs Purchased

(1b)

(Volumes)

(Survey Question Number)

(1)

(1b.i)

95,451

76,638

48,416

Mean

4,416,654

73,125

57,415

34,376

High

$16,250,117$

547,346

304,407

$-45,478$

468,515

$1,853,628$

23,025

499,081,929

$10,785,955$

$8,660,047$

113

4,496

$5,035,290$

113

113

$$
\begin{array}{r}
5,160,340 \\
75,490,151
\end{array}
$$

25,738

25,715

12,564

$1,149,312$

933,306

468,593

Number of Libraries Reporting

GRAND TOTALS
$563,377,383$

$11,626,553$

$9,300,891$

$5,464,033$ 


\section{Collections: Current Serials}

Current Serials Total
Current Serials

Purchased
Current Serials

Purchased

Electronic
Current Serials

Purchased

Print

(Survey Question Number)

(4)

(4a)

(4a.i)

(4a.ii)

INSTITUTION

ALABAMA

ALBERTA

ARIZONA

ARIZONA STATE

AUBURN

BOSTON

BOSTON COLLEGE

BRIGHAM YOUNG

BRITISH COLUMBIA

BROWN

CALIFORNIA BERKELEY

CALIFORNIA DAVIS

CALIFORNIA IRVINE

CALIFORNIA LOS ANGELES

CALIFORNIA RIVERSIDE

CALIFORNIA SAN DIEGO

CALIFORNIA SANTA BARBARA

CASE WESTERN RESERVE

CHICAGO

CINCINNATI

COLORADO

COLORADO STATE

COLUMBIA

CONNECTICUT

CORNELL

DARTMOUTH

DELAWARE

DUKE

EMORY

FLORIDA

FLORIDA STATE

GEORGE WASHINGTON

GEORGETOWN

GEORGIA

GEORGIA TECH

GUELPH

HARVARD

HAWAII

HOUSTON
Notes

bGLM+

G+

bGLM+

BGL+

bG+

LM+

L+

L+

G+

bG+

GL+

GLM+

bGM+

LM+

G+

bGM+

G+

GLM+

bGLM+

bGLM+

bGL+

G+

GLM+

BGLM+

BGL+

$\mathrm{GM}+$

G+

bLM+

BGLM+

BbGLM+

BbLM+

GLM+

bGLM+

GL+

G+

BG+

GLM+

GLM+

bL+
69,426

50,281

88,737

87,566

37,980

56,113

31,465

69,361

64,669

60,499

87,876

59,463

51,623

76,248

89,811

52,535

36,990

66,625

76,607

86,363

55,519

36,133

133,831

61,812

98,000

68,661

29,246

62,639

54,295

71,336

62,093

76,810

61,257

80,748

44,875

11,986

110,628

55,925
48,206

36,793

85,960

66,037

14,030

50,529

21,947

58,466

64,669

43,144

82,162

42,199

34,107

UA/NA

71,266

33,938

27,333

57,889

38,057

70,504

38,140

21,688

102,053

32,801

UA/NA

62,301

20,665

47,819

36,651

47,449

46,054

67,881

52,212

56,454

12,515

11,486

UA/NA

41,356

46,259
UA/NA

27,901

76,470

61,145

13,239

44,392

16,290

36,126

46,657

39,824

37,724

31,636

30,213

UA/NA

69,223

29,706

21,151

52,179

21,733

61,441

35,089

19,876

76,604

27,187

UA/NA

53,172

18,604

35,376

26,487

24,518

42,622

62,236

43,636

48,691

8,620

9,389

UA/NA

30,603

40,867
UA/NA

8,892

9,490

4,892

791

6,137

5,657

22,340

18,012

3,320

44,438

10,563

3,894

UA/NA

2,043

4,232

6,182

5,710

16,324

9,063

3,051

1,812

25,449

5,614

UA/NA

9,129

2,061

12,443

10,164

22,931

3,432

5,645

8,576

7,763

3,895

2,097

UA/NA

10,753

5,392 


\section{Collections: Current Serials}

Current Serials

Not Purchased

(4b)
Current Serials

Not Purchased Consortial
Current Serials Not Purchased

Freely Accessible
Current Serials

Not Purchased Print
Current Serials

Not Purchased Gov Docs

(4b.i)

(4b.ii)

(4b.iii)

(4b.iv) (Survey Question Number)

INSTITUTION

\begin{tabular}{|c|c|c|c|c|c|}
\hline 21,220 & $\mathrm{UA} / \mathrm{NA}$ & $\mathrm{UA} / \mathrm{NA}$ & UA/NA & UA/NA & ALABAMA \\
\hline 13,488 & 0 & 0 & 13,488 & 0 & ALBERTA \\
\hline 2,777 & $\mathrm{UA} / \mathrm{NA}$ & UA/NA & UA/NA & $\mathrm{UA} / \mathrm{NA}$ & ARIZONA \\
\hline 21,529 & 0 & 3,426 & 523 & 17,580 & ARIZONA STATE \\
\hline 23,950 & 10,732 & 5,680 & 857 & 6,681 & AUBURN \\
\hline 5,584 & 1,906 & 3,606 & 43 & 29 & BOSTON \\
\hline 9,518 & 5,233 & 4,260 & 25 & $\mathrm{UA} / \mathrm{NA}$ & BOSTON COLLEGE \\
\hline 10,895 & 0 & 5,550 & 5,095 & 250 & BRIGHAM YOUNG \\
\hline UA/NA & $\mathrm{UA} / \mathrm{NA}$ & $\mathrm{UA} / \mathrm{NA}$ & UA/NA & UA/NA & BRITISH COLUMBIA \\
\hline 17,355 & 0 & 5,661 & 805 & 10,889 & BROWN \\
\hline 5,714 & 0 & 74 & 5,640 & 0 & CALIFORNIA BERKELEY \\
\hline 17,264 & 0 & 11,337 & 3,683 & 2,244 & CALIFORNIA DAVIS \\
\hline 17,516 & 0 & 3,844 & 245 & 13,427 & CALIFORNIA IRVINE \\
\hline UA/NA & $\mathrm{UA} / \mathrm{NA}$ & $\mathrm{UA} / \mathrm{NA}$ & UA/NA & $\mathrm{UA} / \mathrm{NA}$ & CALIFORNIA LOS ANGELES \\
\hline 18,545 & 9,875 & 289 & 112 & 8,269 & CALIFORNIA RIVERSIDE \\
\hline 18,597 & 4,020 & 11,364 & 845 & 2,368 & CALIFORNIA SAN DIEGO \\
\hline 9,657 & 0 & 2,289 & 1,497 & 5,871 & CALIFORNIA SANTA BARBARA \\
\hline 8,736 & 72 & 4,215 & 70 & 4,379 & CASE WESTERN RESERVE \\
\hline 38,550 & 2,234 & 15,069 & 10,384 & 10,863 & CHICAGO \\
\hline 15,859 & 0 & 8,505 & 41 & 7,313 & CINCINNATI \\
\hline 17,379 & 0 & 4,859 & 26 & 12,494 & COLORADO \\
\hline 14,445 & 0 & 12,681 & 575 & 1,189 & COLORADO STATE \\
\hline 31,778 & 0 & 14,637 & 6,013 & 11,128 & COLUMBIA \\
\hline 29,011 & 18,735 & 8,875 & 262 & 1,139 & CONNECTICUT \\
\hline UA/NA & $\mathrm{UA} / \mathrm{NA}$ & $\mathrm{UA} / \mathrm{NA}$ & UA/NA & $\mathrm{UA} / \mathrm{NA}$ & CORNELL \\
\hline 6,360 & 0 & 3,897 & 1,373 & 1,090 & DARTMOUTH \\
\hline 8,581 & 0 & 1,829 & 293 & 6,459 & DELAWARE \\
\hline 14,820 & 0 & 10,009 & 422 & 4,389 & DUKE \\
\hline 17,644 & 3,045 & 11,796 & 99 & 2,704 & EMORY \\
\hline 23,887 & 17,747 & 2,923 & 3,039 & 178 & FLORIDA \\
\hline 16,039 & 10,188 & 3,564 & 901 & 1,386 & FLORIDA STATE \\
\hline 8,929 & 7,587 & 1,332 & 10 & 0 & GEORGE WASHINGTON \\
\hline 9,045 & $\mathrm{UA} / \mathrm{NA}$ & $\mathrm{UA} / \mathrm{NA}$ & UA/NA & $\mathrm{UA} / \mathrm{NA}$ & GEORGETOWN \\
\hline 24,294 & 490 & 16,397 & 921 & 6,486 & GEORGIA \\
\hline 32,360 & 0 & 7,754 & 1,136 & 23,470 & GEORGIA TECH \\
\hline 500 & UA/NA & 500 & UA/NA & UA/NA & GUELPH \\
\hline UA/NA & $\mathrm{UA} / \mathrm{NA}$ & UA/NA & UA/NA & UA/NA & HARVARD \\
\hline 14,569 & $\mathrm{UA} / \mathrm{NA}$ & $\mathrm{UA} / \mathrm{NA}$ & UA/NA & $\mathrm{UA} / \mathrm{NA}$ & HAWAII \\
\hline 26,516 & 9,685 & 4,123 & 3,476 & 9,232 & HOUSTON \\
\hline
\end{tabular}




\section{Collections: Current Serials}

Current Serials Total
Current Serials

Purchased
Current Serials

Purchased

Electronic
Current Serials

Purchased Print

(Survey Question Number)

(4)

(4a)

(4a.i)

(4a.ii)

INSTITUTION

HOWARD
ILLINOIS CHICAGO

ILLINOIS URBANA

INDIANA

IOWA

IOWA STATE

JOHNS HOPKINS

KANSAS

KENT STATE

KENTUCKY

LAVAL

LOUISIANA STATE

LOUISVILLE

MCGILL

MCMASTER

MANITOBA

MARYLAND

MASSACHUSETTS

MIT

MIAMI

MICHIGAN

MICHIGAN STATE

MINNESOTA

MISSOURI

MONTREAL

NEBRASKA

NEW MEXICO

NEW YORK

NORTH CAROLINA

NORTH CAROLINA STATE

NORTHWESTERN

NOTRE DAME

OHIO

OHIO STATE

OKLAHOMA

OKLAHOMA STATE

OREGON

PENNSYLVANIA

PENNSYLVANIA STATE
Notes

bLM+

M+

bGL+

GLM+ 59,924

bLM+

G+

BbG+ $\quad 105,453$

BbLM+

BbG+

GLM+

G+

GLM+

$\mathrm{BbG}+$

BbGLM+

$\mathrm{GM}+$

G+

G

G+

G+

bGLM+

GLM+

GL+ 98,469

bGLM+

GL+

GLM+

GL+

GLM+

bGLM+

GLM+

G+

BGLM+

bGL+

$\mathrm{BbG}+$

bGLM+

GLM+

BGM+

bGL+

GLM+

BGLM+
62,016

60,015

73,251

37,701

101,738

53,461

75,328

53,584

39,332

42,393

43,906

53,548

74,775

69,457

85,075

38,364

59,574

42,589

71,416

67,960

60,713

61,990

77,933

82,866

41,741

89,239

63,407

53,799

46,650

73,248

88,668
a)

18,295

10,670

7,625

37,912

33,789

4,123

102,120

55,270

46,850

49,490

16,419

33,071

41,760

24,827

16,933

47,315

37,115

10,200

76,065

67,519

8,546

49,212

43,076

6,136

58,662

54,968

3,694

34,333

30,435

3,898

28,276

21,829

6,447

84,991

53,461

UA/NA

UA/NA

58,997

40,504

12,957

38,683

52,177

6,820

26,471

35,053

3,630

22,226

4,245

24,441

17,246

7,195

29,160

25,791

3,369

36,259

31,963

4,296

69,164

64,281

4,883

UA/NA

UA/NA

UA/NA

71,888

56,325

61,518

10,370

24,857

35,513

20,812

51,760

18,917

5,940

34,821

44,357

7,403

23,987

10,834

55,474

49,736

5,738

44,503

34,772

9,731

35,802

28,790

7,012

30,902

29,536

1,366

47,236

30,428

16,808

63,682

57,191

6,491

26,522

24,306

2,216

78,906

69,445

9,461

52,522

47,163

5,359

42,658

37,951

4,707

36,029

29,696

6,333

72,347

17,150

80,912

55,197

19,331 


\section{Collections: Current Serials}

Current Serials

Not Purchased

(4b)
Current Serials

Not Purchased Consortial
Current Serials Not Purchased

Freely Accessible
Current Serials

Not Purchased Print
Current Serials

Not Purchased Gov Docs

(4b.i)

(4b.ii)

(4b.iii)

(4b.iv) (Survey Question Number)

INSTITUTION

\begin{tabular}{|c|c|c|c|c|c|}
\hline 8,392 & 204 & 6,131 & 1,477 & 580 & HOWARD \\
\hline 6,702 & 0 & 6,702 & 0 & 0 & ILLINOIS CHICAGO \\
\hline 8,179 & UA/NA & 7,255 & 132 & 792 & ILLINOIS URBANA \\
\hline 10,434 & 577 & 5,768 & 2,189 & 1,900 & INDIANA \\
\hline 17,682 & 0 & 14,109 & 3,573 & 0 & IOWA \\
\hline 18,880 & 0 & 10,478 & 2,977 & 5,425 & IOWA STATE \\
\hline 29,388 & 0 & 411 & 363 & 28,614 & JOHNS HOPKINS \\
\hline 12,804 & 989 & 4,323 & 2,552 & 4,940 & KANSAS \\
\hline 1,353 & 1,353 & 0 & 0 & 0 & KENT STATE \\
\hline 38,918 & 1,910 & 13,105 & 906 & 22,997 & KENTUCKY \\
\hline 9,425 & 0 & 3,752 & 2,843 & 2,830 & LAVAL \\
\hline 16,747 & UA/NA & UA/NA & UA/NA & $\mathrm{UA} / \mathrm{NA}$ & LOUISIANA STATE \\
\hline 0 & 0 & 0 & 0 & 0 & LOUISVILLE \\
\hline 16,331 & 0 & 13,656 & 2,675 & 0 & MCGILL \\
\hline 14,901 & 0 & 14,013 & 888 & 0 & MCMASTER \\
\hline 12,861 & 0 & 10,031 & 1,516 & 1,314 & MANITOBA \\
\hline 17,952 & 7,669 & 9,476 & 713 & 94 & MARYLAND \\
\hline 14,746 & 6,919 & 424 & 828 & 6,575 & MASSACHUSETTS \\
\hline 17,289 & 0 & 11,526 & 898 & 4,865 & MIT \\
\hline 5,611 & 0 & 5,103 & 421 & 87 & MIAMI \\
\hline $\mathrm{UA} / \mathrm{NA}$ & UA/NA & UA/NA & UA/NA & $\mathrm{UA} / \mathrm{NA}$ & MICHIGAN \\
\hline 26,581 & 4,088 & 6,750 & 1,619 & 14,124 & MICHIGAN STATE \\
\hline 28,750 & 17,698 & 0 & 11,052 & 0 & MINNESOTA \\
\hline 13,507 & 6,588 & 5,369 & 991 & 559 & MISSOURI \\
\hline 7,814 & 0 & 4,914 & 2,515 & 385 & MONTREAL \\
\hline 7,768 & 0 & 0 & 2,391 & 5,377 & NEBRASKA \\
\hline 15,942 & 1,940 & 6,392 & 527 & 7,083 & NEW MEXICO \\
\hline 23,457 & 0 & 12,480 & 932 & 10,045 & NEW YORK \\
\hline 24,911 & 3,236 & 2,678 & 8,939 & 10,058 & NORTH CAROLINA \\
\hline 31,088 & 14,443 & 6,377 & 878 & 9,390 & NORTH CAROLINA STATE \\
\hline 30,697 & UA/NA & UA/NA & UA/NA & UA/NA & NORTHWESTERN \\
\hline 19,184 & UA/NA & UA/NA & UA/NA & UA/NA & NOTRE DAME \\
\hline 15,219 & 0 & 3,833 & 107 & 11,279 & $\mathrm{OHIO}$ \\
\hline 10,333 & 0 & 3,613 & 5,299 & 1,421 & OHIO STATE \\
\hline 10,885 & 1,153 & 3,625 & 61 & 6,046 & OKLAHOMA \\
\hline 11,141 & 1,516 & 8,627 & 0 & 998 & OKLAHOMA STATE \\
\hline 10,621 & 2,660 & 5,181 & 1,124 & 1,656 & OREGON \\
\hline UA/NA & UA/NA & UA/NA & UA/NA & UA/NA & PENNSYLVANIA \\
\hline 7,756 & 1,095 & 90 & 1,482 & 5,089 & PENNSYLVANIA STATE \\
\hline
\end{tabular}




\section{Collections: Current Serials}

Current Serials Total
Current Serials

Purchased
Current Serials

Purchased

Electronic
Current Serials

Purchased Print

(Survey Question Number)

(4)

(4a)

(4a.i)

(4a.ii)

\section{INSTITUTION}

PITTSBURGH

PRINCETON

PURDUE

QUEENS

RICE

ROCHESTER

RUTGERS

SASKATCHEWAN

SOUTH CAROLINA

SOUTHERN CALIFORNIA

SOUTHERN ILLINOIS

SUNY-ALBANY

SUNY-BUFFALO

SUNY-STONY BROOK

SYRACUSE

TEMPLE

TENNESSEE

TEXAS

TEXAS A\&M

TEXAS TECH

TORONTO

TULANE

UTAH

VANDERBILT

VIRGINIA

VIRGINIA TECH

WASHINGTON

WASHINGTON STATE

WASHINGTON U.-ST. LOUIS

WATERLOO

WAYNE STATE

WESTERN ONTARIO

WISCONSIN

YALE

YORK

BOSTON PUBLIC

CISTI

CENTER FOR RESEARCH LIBRARIES

LIBRARY OF CONGRESS
Notes

BGLM+

bG+

G+

$\mathrm{LM}+$

G+

$\mathrm{bM}+$

BGL+

GLM+

$\mathrm{LM}+$

BGLM+

$\mathrm{LM}+$

G+

GLM+

$\mathrm{M}+$

GL+

BbGLM+

BbGLM+

GL+

$\mathrm{BbG}+$

BLM+

$\mathrm{B}+$

BLM+

LM

$\mathrm{LM}+$

GLM+

G

GLM+

BG+

bGLM+

G+

$+\quad 45,400$

GL+

GLM+

LM+ $\quad 110,802$

BGL+

G

$+\quad 13,591$

+ UA/NA

bG+
46,539

173,582

59,141

51,746

55,646

72,352

28,637

72,623

32,157

63,715

47,871

54,874

80,431

63,423

60,586

56,991

86,737

66,750

76,473

48,777

47,211

97,800

22,765

61,979

25,264

88,635

23,705

58,715

76,808

5,114

173,582
34,443
a)

(4.i)

$\begin{array}{rrr}49,916 & 42,813 & 7,103 \\ 50,327 & 39,447 & 10,880 \\ 28,822 & 24,537 & 4,285\end{array}$

7,103

0,880

4,285

$\begin{array}{lll}48,829 & 40,828 & 8,001\end{array}$

$\begin{array}{lll}56,806 & 53,027 & 3,779\end{array}$

$23,705 \quad 20,520 \quad 3,185$

$\begin{array}{lll}23,705 & 20,520 & 6,889\end{array}$

$\begin{array}{ll}21,485 & 19,416 \\ 14,629 & 2,069\end{array}$

$\begin{array}{lll}14,629 & 8,702 & 5,927\end{array}$

$\begin{array}{lll}70,066 & 61,514 & 8,552\end{array}$

$\begin{array}{lrr}37,341 & 30,033 & 7,308\end{array}$

$47,665 \quad 35,973 \quad 11,692$

$56,503 \quad 48,402 \quad 8,101$

$\begin{array}{ll}53,900 & 46,477\end{array}$

$25,206 \quad 3,260 \quad 3,946$

$46,976 \quad 42,871 \quad 4,105$

$\begin{array}{ll}34,720 & 29,398\end{array}$

$37,342 \quad 29,401 \quad 7,941$

$52,540 \quad 42,996 \quad 9,544$

$51,580 \quad 46,858 \quad 4,722$

$\begin{array}{lll}49,550 & 38,089 & 11,461\end{array}$

47,295 $\quad 37,161 \quad 10,134$

$\begin{array}{lll}27,168 & 13,701 & 13,467\end{array}$

$33,483 \quad 29,799 \quad 3,684$

$41,440 \quad 37,646 \quad 3,794$

$\begin{array}{lr}11,917 & 9,302 \\ 2,615\end{array}$

UA/NA UA/NA UA/NA

$\begin{array}{lll}69,400 & 63,893 & 5,507\end{array}$

$\begin{array}{lll}16,332 & 11,847 & 4,485\end{array}$

$\begin{array}{lll}34,808 & 31,518 & 3,290\end{array}$

$55,094 \quad 42,578 \quad 12,516$

$\begin{array}{lll}49,626 & 39,486 & 10,140\end{array}$

$\begin{array}{rrr}49,626 & 39,486 & 10,140 \\ 103,444 & 61,326 & 42,118\end{array}$

$\begin{array}{lll}39,608 & 32,204 & 7,404\end{array}$

$\begin{array}{rrr}4,714 & 164 & 4,550 \\ 12,460 & 9,251 & 3,209\end{array}$

UA/NA UA/NA UA/NA

$\begin{array}{lll}66,462 & 23,528 & 42,934\end{array}$




\section{Collections: Current Serials}

Current Serials

Not Purchased

(4b)

Current Serials

Not Purchased Consortial
Current Serials

Not Purchased

Freely Accessible
Current Serials

Not Purchased Print
Current Serials

Not Purchased Gov Docs

(4b.ii)

(4b.iii)

(4b.iv) (Survey Question Number)

INSTITUTION

\begin{tabular}{|c|c|c|c|c|c|}
\hline 9,225 & 0 & 7,715 & 1,307 & 203 & PITTSBURGH \\
\hline 1,419 & 0 & 1,000 & 100 & 319 & PRINCETON \\
\hline 11,272 & UA/NA & UA/NA & UA/NA & UA/NA & PURDUE \\
\hline 6,817 & 0 & 6,285 & 532 & 0 & QUEENS \\
\hline 15,546 & 0 & 10,987 & 210 & 4,349 & RICE \\
\hline 4,932 & 0 & 4,073 & 859 & 0 & ROCHESTER \\
\hline 23,116 & 7,357 & 6,937 & 477 & 8,345 & RUTGERS \\
\hline 10,672 & 0 & 10,373 & 299 & 0 & SASKATCHEWAN \\
\hline 49,086 & 47,693 & 780 & 276 & 337 & SOUTH CAROLINA \\
\hline 35,678 & 1,136 & 12,854 & 1,813 & 19,875 & SOUTHERN CALIFORNIA \\
\hline 10,530 & 592 & 9,560 & 378 & $\mathrm{UA} / \mathrm{NA}$ & SOUTHERN ILLINOIS \\
\hline 7,209 & 1,852 & 2,728 & 0 & 2,629 & SUNY-ALBANY \\
\hline 23,928 & 7 & 14,080 & 3,192 & 6,649 & SUNY-BUFFALO \\
\hline 9,523 & 2,502 & 6,865 & 128 & 28 & SUNY-STONY BROOK \\
\hline 9,237 & 0 & 3,937 & 1,802 & 3,498 & SYRACUSE \\
\hline 13,610 & 0 & 12,345 & 396 & 869 & TEMPLE \\
\hline 15,832 & 7,253 & 119 & 55 & 8,405 & TENNESSEE \\
\hline 19,649 & 0 & 10,566 & 4,549 & 4,534 & TEXAS \\
\hline 34,197 & 7,923 & 15,446 & 590 & 10,238 & TEXAS A\&M \\
\hline 15,170 & 0 & 14,965 & 205 & 0 & TEXAS TECH \\
\hline 26,923 & 0 & 24,282 & 2,641 & 0 & TORONTO \\
\hline 25,269 & 0 & 24,562 & 707 & 0 & TULANE \\
\hline 21,609 & 17,039 & 3,667 & 700 & 203 & UTAH \\
\hline 13,728 & 5,262 & 4,557 & 389 & 3,520 & VANDERBILT \\
\hline 56,360 & 0 & 51,513 & 506 & 4,341 & VIRGINIA \\
\hline 10,848 & 9,698 & 300 & 150 & 700 & VIRGINIA TECH \\
\hline UA/NA & UA/NA & UA/NA & UA/NA & $\mathrm{UA} / \mathrm{NA}$ & WASHINGTON \\
\hline UA/NA & UA/NA & UA/NA & UA/NA & UA/NA & WASHINGTON STATE \\
\hline 19,235 & 2,246 & 13,194 & 1,537 & 2,258 & WASHINGTON U.-ST. LOUIS \\
\hline 7,373 & 0 & 7,373 & 0 & 0 & WATERLOO \\
\hline 10,592 & 5,060 & 3,532 & 422 & 1,578 & WAYNE STATE \\
\hline 3,621 & 0 & 1,158 & 1,676 & 787 & WESTERN ONTARIO \\
\hline 27,182 & 0 & 23,023 & 3,181 & 978 & WISCONSIN \\
\hline 7,358 & 1,271 & 4,167 & 1,920 & 0 & YALE \\
\hline 6,931 & 0 & 5,602 & 853 & 476 & YORK \\
\hline 400 & 0 & 0 & 0 & 400 & BOSTON PUBLIC \\
\hline 1,131 & 0 & 553 & 578 & 0 & CISTI \\
\hline UA/NA & UA/NA & UA/NA & UA/NA & UA/NA & CENTER FOR RESEARCH LIBRARIES \\
\hline 107,120 & 0 & 4,000 & 103,120 & 0 & LIBRARY OF CONGRESS \\
\hline
\end{tabular}




\section{Collections: Current Serials}

Current Serials

Total
Current Serials

Purchased
Current Serials

Purchased

Electronic
Current Serials

Purchased

Print

(Survey Question Number)

(4)

(4a)

(4a.i)

(4a.ii)

INSTITUTION

Notes

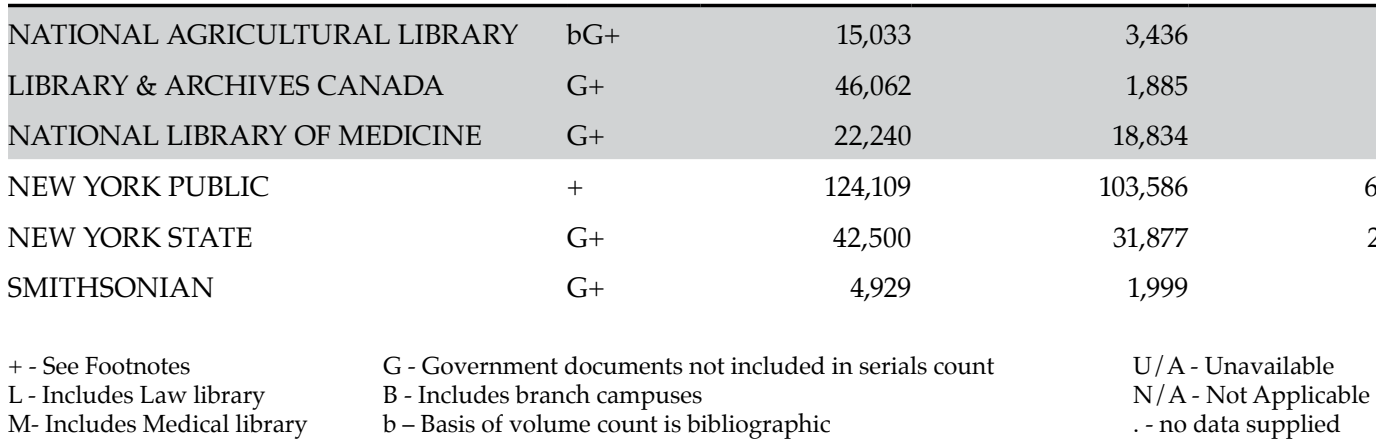

Summary Data

Current Serials

Total
Current Serials

Purchased
Current Serials

Purchased

Electronic
2,288

1,620

14,963

38,351

7,962

1,562

437

38,351

23,915 7,962

1,562

- Basis of volume count is bibliographic
- no data supplied
(4)

\section{UNIVERSITY LIBRARIES}

Mean

Median

High

Low

Totals

Number of Libraries Reporting

\section{NONUNIVERSITY LIBRARIES}

Median

Totals

Number of Libraries Reporting

$$
\begin{array}{r}
63,306 \\
61,812 \\
133,831 \\
11,986 \\
7,153,577
\end{array}
$$

11,986

113
447,160

9

245,253

127,814

117,439

9

$5,245,516$

$4,049,324$

$1,062,995$

GRAND TOTALS

Current Serials

Purchased

Print 


\section{Collections: Current Serials}

Current Serials

Not Purchased

(4b)
Current Serials

Not Purchased Consortial
Current Serials Not Purchased

Freely Accessible
Current Serials

Not Purchased Print
Current Serials

Not Purchased

Gov Docs
(4b.i)
(4b.ii)
(4b.iii)
(4b.iv) (Survey Question Number)

INSTITUTION

\begin{tabular}{rrrrrr}
\hline 11,597 & 0 & 2,627 & 8,970 & 0 & NATIONAL AGRICULTURAL LIBRARY \\
44,177 & 0 & 0 & 44,177 & 0 & LIBRARY \& ARCHIVES CANADA \\
3,406 & 0 & 1,196 & 1,896 & 314 & NATIONAL LIBRARY OF MEDICINE \\
20,523 & UA/NA & UA/NA & UA/NA & UA/NA & NEW YORK PUBLIC \\
10,623 & 0 & 0 & 5,741 & 4,882 & NEW YORK STATE \\
2,930 & 0 & 0 & 2,628 & 302 & SMITHSONIAN
\end{tabular}

Current Serials Not Purchased

Current Serials Not Purchased Consortial

\section{Summary Data}

Current Serials

Not Purchased

Freely Accessible
Current Serials Not Purchased Print
Current Serials

Not Purchased

Gov Docs (4b)

16,294

14,820

56,360

0

$1,710,837$

105 (4b.i)

3,079

0

47,693

0

292,478

95 (4b.ii)

7,372

5,661

51,513

0

715,082

97 (4b.iii)

1,631

849

13,488

0

156,617

96 (4b.iv) (SurveyQuestionNumber)

UNIVERSITY LIBRARIES

Mean

Median

High

Low

Totals

421,149

94

Number of Libraries Reporting
151

5,898

8

NONUNIVERSITY LIBRARIES

Median

Totals

167,110

8

Totals

Number of Libraries Reporting

$1,912,744$

292,478

723,458

323,727

GRAND TOTALS 


\section{Collections: Other Materials}

Microform Government Computer Manuscripts Cartographic

Units

(6)

(7)

(8)

(9)

(10)
Graphic

Materials

(11)

$(12)$
Film and

(13)

INSTITUTION

Notes

\begin{tabular}{|c|c|c|c|c|c|c|c|c|c|}
\hline ALABAMA & bGLM+ & $4,096,093$ & 926,705 & 8,448 & 34,231 & 311,178 & 189,324 & 23,546 & 7,118 \\
\hline ALBERTA & G+ & $3,738,979$ & 0 & 9,260 & 22,461 & $1,546,176$ & UA/NA & UA/NA & $\mathrm{UA} / \mathrm{NA}$ \\
\hline ARIZONA & bGLM+ & $6,282,673$ & UA/NA & 19,683 & 10,639 & 268,050 & 358,788 & 43,257 & 29,272 \\
\hline ARIZONA STATE & BGL+ & $7,588,679$ & 664,596 & 7,603 & 25,229 & 235,497 & $1,263,639$ & 67,179 & 36,473 \\
\hline AUBURN & bG+ & $2,718,458$ & 237,752 & 3,442 & 10,524 & 152,412 & 75,327 & 115,903 & 5,517 \\
\hline BOSTON & $\mathrm{LM}+$ & $4,700,124$ & UA/NA & 2,237 & 50,422 & 1,700 & 17,700 & 73,887 & 8,074 \\
\hline BOSTON COLLEGE & $\mathrm{L}+$ & 4,190,134 & 215,703 & 6,253 & 8,253 & 17,940 & 127,575 & 21,772 & 23,566 \\
\hline BRIGHAM YOUNG & $\mathrm{L}+$ & $3,874,728$ & 423,514 & UA/NA & 45,643 & 257,886 & $1,275,047$ & 240,359 & 39,055 \\
\hline BRITISH COLUMBIA & G+ & $5,291,774$ & 0 & 27,748 & 12,877 & 613,565 & $1,006,226$ & 99,883 & 36,536 \\
\hline BROWN & bG+ & $1,966,000$ & 0 & 5,563 & 12,262 & 153,231 & 950,397 & 77,923 & 9,563 \\
\hline CALIFORNIA BERKELEY & GL+ & $7,128,372$ & 492,722 & 20,386 & 86,293 & 441,673 & $9,653,424$ & 94,697 & 50,034 \\
\hline CALIFORNIA DAVIS & GLM+ & $4,241,335$ & 79,264 & 13,338 & 18,027 & 263,516 & 235,525 & 12,911 & 4,336 \\
\hline CALIFORNIA IRVINE & bGM+ & $2,893,477$ & 152,779 & 11,275 & 4,240 & 11,300 & 71,661 & 13,079 & 13,041 \\
\hline CALIFORNIA LOS ANGELES & $\mathrm{LM}+$ & $6,218,665$ & 72,716 & 16,286 & 69,960 & 636,051 & $4,143,107$ & 269,704 & 35,317 \\
\hline CALIFORNIA RIVERSIDE & G+ & $2,280,683$ & 299,797 & 11,854 & 4,411 & 106,673 & 22,100 & 21,940 & 9,799 \\
\hline CALIFORNIA SAN DIEGO & bGM+ & $3,309,102$ & 0 & 17,143 & 14,053 & 114,780 & 318,820 & 124,362 & 19,863 \\
\hline CALIFORNIA SANTA BARBARA & G+ & $3,771,838$ & 451,767 & 13,229 & 17,095 & $4,461,772$ & 1,824 & 134,935 & 11,207 \\
\hline CASE WESTERN RESERVE & GLM+ & $2,601,135$ & 29,017 & 8,459 & 14,690 & 65,080 & 72,556 & 34,356 & 22,060 \\
\hline CHICAGO & bGLM+ & $3,114,979$ & UA/NA & 12,590 & 39,296 & 435,570 & 0 & 56,995 & 7,751 \\
\hline CINCINNATI & bGLM+ & $3,371,800$ & 30,140 & 13,998 & 26,142 & 172,474 & 302,023 & 64,835 & 23,433 \\
\hline COLORADO & bGL+ & $6,993,960$ & 963,779 & 12,156 & 38,518 & 218,069 & 970,698 & 69,010 & 16,697 \\
\hline COLORADO STATE & G+ & $1,159,032$ & UA/NA & 2,511 & 7,084 & 39,262 & 4,449 & 778 & 863 \\
\hline COLUMBIA & GLM+ & $6,345,302$ & 175,665 & 37,092 & 243,114 & 139,128 & 864,228 & 106,393 & 37,218 \\
\hline CONNECTICUT & BGLM+ & $4,320,791$ & 0 & 4,075 & 27,261 & 232,404 & 2,416 & 60,634 & 11,560 \\
\hline CORNELL & BGL+ & $8,507,563$ & 0 & 19,495 & 65,673 & 253,439 & 53,026 & 121,293 & 39,842 \\
\hline DARTMOUTH & $\mathrm{GM}+$ & $2,605,009$ & 0 & 11,150 & 23,168 & 191,091 & 490,828 & 37,193 & 18,544 \\
\hline DELAWARE & G+ & $3,443,953$ & 451,186 & 357,153 & 5,119 & 126,724 & 781 & 1,983 & 19,878 \\
\hline DUKE & bLM+ & $4,313,350$ & $1,257,457$ & 61,520 & 55,103 & 132,901 & 925,054 & 61,665 & 76,203 \\
\hline EMORY & BGLM+ & $6,254,488$ & 256,899 & 12,078 & 23,129 & 17,418 & 2,959 & 55,613 & 40,647 \\
\hline FLORIDA & BbGLM+ & $7,901,639$ & $1,241,342$ & 8,315 & 8,840 & 853,546 & 191,323 & 23,337 & 44,765 \\
\hline FLORIDA STATE & BbLM+ & $9,109,694$ & 857,378 & 9,838 & 8,741 & 173,350 & 6,590 & 62,592 & 19,459 \\
\hline GEORGE WASHINGTON & GLM+ & 2,946,196 & 0 & 891 & 22,794 & 18,837 & 170,429 & 10,642 & 17,367 \\
\hline GEORGETOWN & bGLM+ & $4,026,834$ & 9,385 & 7,640 & 18,406 & 1,963 & 377,471 & 21,785 & 32,728 \\
\hline GEORGIA & GL+ & $6,632,493$ & $1,066,004$ & 21,866 & 59,023 & 660,644 & $1,647,480$ & 134,841 & 159,524 \\
\hline GEORGIA TECH & G+ & $4,627,573$ & 0 & 15,584 & 6,696 & 198,213 & 111,267 & 1,866 & 6,177 \\
\hline GUELPH & BG+ & $2,346,336$ & 0 & 3,198 & 13,560 & 75,292 & 6,882 & 12,468 & $\mathrm{UA} / \mathrm{NA}$ \\
\hline HARVARD & GLM+ & $9,814,796$ & UA/NA & $\mathrm{UA} / \mathrm{NA}$ & $\mathrm{UA} / \mathrm{NA}$ & UA/NA & $\mathrm{UA} / \mathrm{NA}$ & UA/NA & $\mathrm{UA} / \mathrm{NA}$ \\
\hline HAWAII & GLM+ & $3,517,793$ & 0 & 5,341 & 7,867 & 93,955 & 3,116 & 35,055 & 34,623 \\
\hline HOUSTON & $\mathrm{bL}+$ & $5,985,299$ & 680,971 & 2,721 & 4,971 & 5,704 & 526 & 17,218 & 7,292 \\
\hline
\end{tabular}




\section{Collections: Other Materials}

Microform Government Computer Manuscripts Cartographic

Units - Documents

(6)

(7)

(8)

(9)

(10)
Graphic

Materials

(11)

$(12)$
Film and

(13)

(13)

INSTITUTION

Notes

\begin{tabular}{|c|c|c|c|c|c|c|c|c|c|}
\hline HOWARD & bLM+ & $4,234,651$ & 6,759 & 326 & 20,810 & 40 & 160,211 & 25,369 & 8,548 \\
\hline ILLINOIS CHICAGO & $\mathrm{M}+$ & 4,197,939 & 793,177 & $\mathrm{UA} / \mathrm{NA}$ & 33,733 & 204,768 & $2,108,579$ & 7,846 & 12,407 \\
\hline ILLINOIS URBANA & bGL+ & $9,636,471$ & 0 & 14,225 & 46,118 & 674,574 & 90,822 & 159,474 & 29,575 \\
\hline INDIANA & GLM+ & $6,882,420$ & 383,266 & 79,695 & 36,423 & 524,525 & $3,026,857$ & 275,313 & 66,559 \\
\hline IOWA & bLM+ & $\mathrm{UA} / \mathrm{NA}$ & $\mathrm{UA} / \mathrm{NA}$ & $\mathrm{UA} / \mathrm{NA}$ & 16,969 & 423,215 & $\mathrm{UA} / \mathrm{NA}$ & 29,665 & 35,113 \\
\hline IOWA STATE & G+ & $3,521,259$ & 0 & 10,911 & 17,203 & 98,676 & 904,186 & 16,453 & 54,011 \\
\hline JOHNS HOPKINS & $\mathrm{BbG}+$ & $4,394,653$ & 1,324 & 6,158 & 13,313 & 217,136 & 69,429 & 31,408 & 12,756 \\
\hline KANSAS & BbLM+ & $3,657,049$ & 739,024 & 13,835 & 55,683 & 337,719 & $3,407,468$ & 38,948 & 25,114 \\
\hline KENT STATE & $\mathrm{BbG}+$ & UA/NA & 0 & 85,000 & 11,364 & 8,255 & 13,936 & 16,954 & 14,152 \\
\hline KENTUCKY & GLM+ & $6,471,766$ & $1,036,210$ & 8,209 & 26,780 & 261,629 & $2,148,672$ & 50,914 & 42,964 \\
\hline LAVAL & G+ & $1,299,734$ & 0 & 7,017 & 0 & 302,853 & 214,552 & 23,367 & 29,184 \\
\hline LOUISIANA STATE & GLM+ & $9,014,560$ & 590,485 & 9,828 & 23,877 & 454,880 & $\mathrm{UA} / \mathrm{NA}$ & 24,288 & 4,250 \\
\hline LOUISVILLE & $\mathrm{BbG}+$ & $2,219,718$ & 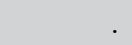 & & $20,869,940$ & 22,340 & $1,514,744$ & 33,302 & 10,249 \\
\hline MCGILL & BbGLM+ & $1,848,185$ & 784,146 & 10,369 & 3,658 & 237,049 & 322,655 & 53,102 & 15,342 \\
\hline MCMASTER & GM+ & $1,599,937$ & 0 & 1,860 & 12,966 & 146,826 & 253 & 29,018 & 3,062 \\
\hline MANITOBA & G+ & $1,473,614$ & 222,243 & $\mathrm{UA} / \mathrm{NA}$ & 12,947 & 105,734 & 269,825 & 29,903 & 10,446 \\
\hline MARYLAND & G & $5,832,845$ & 199,706 & 7,829 & 42,156 & 224,015 & 363,467 & 341,485 & 47,782 \\
\hline MASSACHUSETTS & G+ & $2,584,531$ & 0 & 1,806 & 15,153 & 99,810 & 250,060 & 20,743 & 4,487 \\
\hline MIT & G+ & $2,416,804$ & UA/NA & 59,069 & 24,280 & 126,514 & 452,377 & 32,191 & 6,607 \\
\hline MIAMI & bGLM+ & $3,993,799$ & 0 & 3,880 & 17,932 & 58,863 & 12,746 & 57,028 & 16,468 \\
\hline MICHIGAN & GLM+ & UA/NA & 0 & $\mathrm{UA} / \mathrm{NA}$ & 47,306 & 349,447 & $20,815,850$ & 60,086 & 42,131 \\
\hline MICHIGAN STATE & GL+ & $6,776,737$ & UA/NA & 25,367 & 2,750 & 232,107 & 1,817 & 68,280 & 6,181 \\
\hline MINNESOTA & bGLM+ & $7,008,203$ & $2,640,164$ & 17,711 & 80,729 & 445,773 & UA/NA & UA/NA & $\mathrm{UA} / \mathrm{NA}$ \\
\hline MISSOURI & GL+ & $8,118,672$ & $1,681,883$ & 2,320 & 11,853 & 273,036 & 630 & 25,578 & 5,713 \\
\hline MONTREAL & GLM+ & $1,547,142$ & 0 & 9,259 & 0 & 13,171 & 115,696 & 65,449 & 15,269 \\
\hline NEBRASKA & GL+ & $4,687,095$ & 552,672 & 8,205 & 10,620 & 169,586 & 103,900 & 36,053 & 3,469 \\
\hline NEW MEXICO & GLM+ & $4,721,312$ & 663,556 & 76,982 & 15,813 & 233,689 & $1,094,244$ & 31,165 & 10,040 \\
\hline NEW YORK & bGLM+ & $5,561,308$ & 482,172 & 6,320 & 35,777 & 383 & $2,400,299$ & 107,601 & 50,651 \\
\hline NORTH CAROLINA & GLM+ & $5,142,758$ & $1,625,932$ & 12,743 & 39,911 & 297,079 & $1,623,335$ & 317,383 & 64,831 \\
\hline NORTH CAROLINA STATE & G+ & $5,446,201$ & 0 & 406,619 & 13,791 & 49,225 & 128,463 & 4,683 & 24,650 \\
\hline NORTHWESTERN & BGLM+ & $4,554,601$ & 7,808 & 15,407 & 17,608 & 208,383 & 611,000 & 64,214 & 32,522 \\
\hline NOTRE DAME & bGL+ & $3,958,901$ & 0 & 6,646 & $\mathrm{UA} / \mathrm{NA}$ & 20,779 & 229,998 & 25,558 & 11,598 \\
\hline $\mathrm{OHIO}$ & $\mathrm{BbG}+$ & $2,000,195$ & $\mathrm{UA} / \mathrm{NA}$ & 4,658 & 7,300 & 45,995 & 182,198 & 32,628 & 36,106 \\
\hline OHIO STATE & bGLM+ & $6,059,429$ & 832 & 14,315 & 35,181 & 230,474 & $2,468,362$ & 81,360 & $\mathrm{UA} / \mathrm{NA}$ \\
\hline OKLAHOMA & GLM+ & $4,465,689$ & 35,360 & 5,366 & 18,258 & 155,851 & $1,748,835$ & 4,574 & 8,672 \\
\hline OKLAHOMA STATE & BGM+ & $4,533,703$ & 180,337 & 15,622 & 10,641 & 320,214 & 179,562 & 13,779 & 17,835 \\
\hline OREGON & bGL+ & $4,159,767$ & 268,640 & 3,012 & 74,329 & 780,424 & $1,319,929$ & 77,990 & 28,999 \\
\hline PENNSYLVANIA & GLM+ & $4,147,587$ & UA/NA & 249 & 18,000 & 138,602 & 753,377 & 91,180 & 20,219 \\
\hline PENNSYLVANIA STATE & BGLM+ & $6,933,343$ & 0 & 11,059 & 31,945 & 442,678 & 597,846 & 82,704 & 171,402 \\
\hline
\end{tabular}




\section{Collections: Other Materials}

Microform Government Computer Manuscripts Cartographic

Units Documents

Files and Archives Materials

Graphic Audio Film and

(6) (7)

(8)

(9)

(10)

(11)

(12)

(13)

INSTITUTION

Notes

\begin{tabular}{|c|c|c|c|c|c|c|c|c|c|}
\hline PITTSBURGH & BGLM+ & $5,442,379$ & 0 & 58,519 & 31,528 & 124,412 & 812,701 & 115,315 & 95,114 \\
\hline PRINCETON & bG+ & $6,550,035$ & 4,646 & 11,864 & 35,143 & 334,099 & 249,521 & 93,950 & 9,284 \\
\hline PURDUE & G+ & $3,104,959$ & 485,929 & 13,234 & 3,610 & 344,522 & 1,542 & 1,027 & 12,968 \\
\hline QUEENS & LM+ & $3,923,674$ & $1,114,838$ & UA/NA & UA/NA & 158,880 & 28,322 & UA/NA & UA/NA \\
\hline RICE & G+ & $3,276,520$ & 55,896 & 15,682 & 7,926 & 27,167 & 34,900 & 48,219 & 14,060 \\
\hline ROCHESTER & $\mathrm{bM}+$ & $5,120,381$ & 419,912 & 7,325 & 15,123 & 105,411 & 11,812 & 98,294 & 19,054 \\
\hline RUTGERS & BGL+ & $6,048,708$ & $2,668,634$ & 15,524 & 28,016 & UA/NA & UA/NA & UA/NA & $\mathrm{UA} / \mathrm{NA}$ \\
\hline SASKATCHEWAN & GLM+ & $3,087,965$ & 414,618 & 4,009 & 268 & 73,526 & 18,599 & 17,513 & 1,936 \\
\hline SOUTH CAROLINA & LM+ & $5,357,428$ & 944,768 & 4,044 & 9,876 & 323,474 & 250,090 & 38,412 & 17,486 \\
\hline SOUTHERN CALIFORNIA & BGLM+ & $6,329,276$ & 271,720 & 11,598 & 47,940 & 84,494 & $3,192,388$ & 32,048 & 34,755 \\
\hline SOUTHERN ILLINOIS & LM+ & $4,601,724$ & 312,699 & 11,214 & 27,136 & 343,126 & 6,997 & 29,880 & 18,461 \\
\hline SUNY-ALBANY & G+ & $2,896,185$ & 0 & 2,283 & 13,133 & 25,207 & 56,149 & 8,037 & 7,764 \\
\hline SUNY-BUFFALO & GLM+ & $6,120,752$ & 0 & 13,982 & 12,623 & 198,605 & 167,423 & 59,750 & 17,688 \\
\hline SUNY-STONY BROOK & $\mathrm{M}+$ & $3,837,217$ & 347,884 & 862 & 8,065 & 133,173 & 9,457 & 41,822 & 8,221 \\
\hline SYRACUSE & GL+ & $7,491,672$ & 291,548 & 2,705 & 29,909 & 279,602 & 417,766 & 415,469 & 22,417 \\
\hline TEMPLE & BbGLM+ & $3,308,050$ & 24,766 & 3,588 & 38,575 & 121,662 & $10,038,127$ & 23,901 & 10,537 \\
\hline TENNESSEE & BbGLM+ & $4,389,259$ & 4,158 & 3,667 & 13,622 & 392,137 & 14,017 & 25,891 & 19,082 \\
\hline TEXAS & GL+ & $6,628,794$ & 0 & 10,526 & 126,180 & 327,219 & $11,931,928$ & 193,332 & 78,236 \\
\hline TEXAS A\&M & $\mathrm{BbG}+$ & $5,686,474$ & 65,519 & 10,869 & 22,856 & 237,956 & 541,868 & 26,730 & 28,463 \\
\hline TEXAS TECH & BLM+ & $2,726,735$ & 4,222 & 390,368 & 33,532 & 162,427 & $1,649,059$ & 25,367 & 34,682 \\
\hline TORONTO & $\mathrm{B}+$ & $5,491,412$ & UA/NA & 848,299 & 41,416 & 281,760 & 781,676 & 206,148 & 34,177 \\
\hline TULANE & BLM+ & $2,252,124$ & 94,550 & 1,495 & 48,785 & 29,986 & 560 & 7,942 & 4,995 \\
\hline UTAH & LM & $3,638,146$ & 898,343 & 8,206 & 36,178 & 254,189 & $1,658,465$ & 52,085 & 35,458 \\
\hline VANDERBILT & LM+ & $3,044,268$ & 263,092 & 9,839 & 21,413 & 108,744 & 224,321 & 38,135 & 21,696 \\
\hline VIRGINIA & GLM+ & $5,536,709$ & 674,257 & 11,665 & 27,928 & 152,697 & 449,408 & 81,420 & 38,769 \\
\hline VIRGINIA TECH & G & $6,323,119$ & 399,525 & 10,594 & 17,979 & 140,478 & 163,975 & 11,779 & 17,172 \\
\hline WASHINGTON & GLM+ & $7,788,178$ & 0 & 18,448 & 68,413 & 369,521 & $1,335,917$ & 80,395 & 49,249 \\
\hline WASHINGTON STATE & BG+ & $3,938,825$ & 0 & 541 & 19,333 & 2,418 & 369,435 & 15,356 & 35,491 \\
\hline WASHINGTON U.-ST. LOUIS & bGLM+ & $3,448,834$ & 0 & 14,967 & 15,671 & 117,685 & 3 & 42,573 & 61,366 \\
\hline WATERLOO & G+ & $1,710,239$ & 0 & 9,724 & 5,633 & 146,659 & 41 & 413 & 261 \\
\hline WAYNE STATE & + & $3,896,048$ & 388,182 & 5,797 & 74,000 & 56,235 & $2,159,539$ & 46,731 & 30,565 \\
\hline WESTERN ONTARIO & GL+ & $4,062,350$ & 0 & 0 & 11,634 & 15,381 & $1,600,000$ & 49,390 & 1,685 \\
\hline WISCONSIN & GLM+ & $6,350,062$ & 757,805 & 15,978 & 158,397 & 599,282 & $6,280,823$ & 353,608 & 29,588 \\
\hline YALE & $\mathrm{LM}+$ & $10,269,372$ & 0 & 0 & 92,675 & 462,904 & $2,340,314$ & 243,416 & 29,211 \\
\hline YORK & BGL+ & $4,187,860$ & $\mathrm{UA} / \mathrm{NA}$ & 3,177 & 12,346 & 111,980 & 8,647 & 36,227 & 27,025 \\
\hline BOSTON PUBLIC & G & $6,943,244$ & $1,858,078$ & 13 & $\mathrm{UA} / \mathrm{NA}$ & . & . & 65,948 & 125,030 \\
\hline CISTI & + & $5,000,000$ & 0 & 2,050 & 2,231 & $\mathrm{UA} / \mathrm{NA}$ & $\mathrm{UA} / \mathrm{NA}$ & UA/NA & 749 \\
\hline CENTER FOR RESEARCH LIBRARIES & + & $1,901,585$ & $\mathrm{UA} / \mathrm{NA}$ & 1,537 & $\mathrm{UA} / \mathrm{NA}$ & $\mathrm{UA} / \mathrm{NA}$ & $\mathrm{UA} / \mathrm{NA}$ & UA/NA & $\mathrm{UA} / \mathrm{NA}$ \\
\hline LIBRARY OF CONGRESS & $\mathrm{bG}+$ & $16,086,572$ & 0 & 380,648 & 62,778 & $5,357,385$ & $14,470,265$ & $3,005,028$ & $1,207,776$ \\
\hline $\begin{array}{l}\text { NATIONAL AGRICULTURAL } \\
\text { LIBRARY }\end{array}$ & bG+ & $1,100,928$ & 0 & 1,945 & 19,808 & 5,140 & 1,476 & 403 & 4,016 \\
\hline
\end{tabular}




\section{Collections: Other Materials}

Microform Government

Units Documents
Computer Manuscripts Cartographic Files and Archives Materials
Graphic

Materials
Audio

Materials

(12)
Film and Video

(Survey Question Number)

(6)

(7)

(8)

(9)

(10)

(11)

(13)

INSTITUTION

Notes

\begin{tabular}{|c|c|c|c|c|c|c|c|c|c|}
\hline LIBRARY \& ARCHIVES CANADA & G+ & $8,182,821$ & $334,000,000$ & UA/NA & 170,285 & $2,966,064$ & $24,627,186$ & 333,973 & 202,694 \\
\hline NATIONAL LIBRARY OF MEDICINE & G+ & 605,672 & 0 & 2,560 & 4,878 & 0 & 69,285 & UA/NA & 83,837 \\
\hline NEW YORK PUBLIC & + & $6,916,121$ & UA/NA & $\mathrm{UA} / \mathrm{NA}$ & 65,905 & 433,776 & $4,399,640$ & 580,835 & 72,467 \\
\hline NEW YORK STATE & G+ & $8,504,454$ & UA/NA & 8,862 & 29,291 & 211,366 & 71,358 & 56 & 94 \\
\hline SMITHSONIAN & G+ & 190,368 & 0 & 1,763 & 2,124 & 149 & 2,031 & 359 & 583 \\
\hline
\end{tabular}

+ - See Footnotes

L - Includes Law library

M- Includes Medical library
G - Government documents not included in serials count B - Includes branch campuses

$\mathrm{b}$ - Basis of volume count is bibliographic
U/A - Unavailable

N/A - Not Applicable

- no data supplied

\section{Summary Data}

Microform Government Units Documents
Computer Manuscripts Cartographic Files and Archives Materials
Graphic

Materials
Audio

Materials
Film and

Video

(Survey Question Number)

(6)

(7)

(8)

(9)

(10)

(11)

(12)

(13)

\section{UNIVERSITY LIBRARIES}

\section{Mean}

Median

High

Low

Totals

Number of Libraries Reporting

$\begin{array}{rr}4,701,813 & 348,397 \\ 4,277,343 & 123,665 \\ 10,269,372 & 2,668,634 \\ 1,159,032 & 0 \\ 517,199,440 & 34,839,745 \\ 110 & 100\end{array}$

31,751
10,369
848,299
0
$3,333,889$
105

219,053

263,800

18,870

172,474

1,144,173

69,199

26,808

$20,869,940$

$4,461,772$

249,521

40,385

19,661

$20,815,850$

415,469

171,402

0

40

413

261

$24,095,794$

$29,281,799 \quad 122,426,556$

$7,473,500 \quad 2,841,682$

110

111

107

108

106

\section{NONUNIVERSITY LibraRIES}

Median
Totals
Number of Libraries Reporting

GRAND TOTALS

$\begin{array}{rrrrrrrr}5,958,061 & 0 & 1,998 & 24,550 & 211,366 & 71,358 & 65,948 & 72,467 \\ 55,431,765 & 335,858,078 & 399,378 & 357,300 & 8,973,880 & 43,641,241 & 3,986,602 & 1,697,246 \\ 10 & 7 & 8 & 8 & 7 & 7 & 7 & 9 \\ & & & & & & & \\ 572,631,205 & 370,697,823 & 3,733,267 & 24,453,094 & 38,255,679 & 166,067,797 & 11,460,102 & 4,538,928\end{array}$




\section{EXPENDITURES}

Monographs Current Serials Other Library Miscellaneous Total Library

Contract Materials Materials Materials

(15.c)

(15.d)

(15)

(16)

\section{INSTITUTION}

\begin{tabular}{|c|c|c|c|c|c|c|c|}
\hline ALABAMA & bGLM+ & $1,241,164$ & $6,329,417$ & 80,501 & 37,164 & $7,688,246$ & 68,007 \\
\hline ALBERTA & G+ & $6,087,053$ & $10,155,590$ & 0 & 637,488 & $16,880,131$ & 82,715 \\
\hline ARIZONA & bGLM+ & $2,857,568$ & $8,366,628$ & 840,821 & 562,371 & $12,627,388$ & 63,659 \\
\hline ARIZONA STATE & BGL+ & $2,457,075$ & $8,261,537$ & 552,159 & 162,780 & $11,433,551$ & 137,606 \\
\hline AUBURN & bG+ & 568,640 & $5,016,185$ & 44,937 & 0 & $5,629,762$ & 48,116 \\
\hline BOSTON & LM+ & $1,305,954$ & $7,860,431$ & 286,547 & 15,125 & $9,468,057$ & 160,363 \\
\hline BOSTON COLLEGE & $\mathrm{L}+$ & $1,368,393$ & $5,125,794$ & $1,118,387$ & $1,366,735$ & $8,979,309$ & 96,752 \\
\hline BRIGHAM YOUNG & $\mathrm{L}+$ & $3,178,353$ & $7,909,894$ & 64,206 & 155,394 & $11,307,847$ & 116,780 \\
\hline BRITISH COLUMBIA & G+ & $3,296,520$ & $10,404,252$ & 832,745 & 383,713 & $14,917,231$ & 135,630 \\
\hline BROWN & bG+ & $1,934,956$ & $5,593,056$ & 329,890 & 636,358 & $8,494,260$ & 78,959 \\
\hline CALIFORNIA BERKELEY & GL+ & $8,117,984$ & $10,766,841$ & 850,834 & 383,188 & $20,118,847$ & $1,054,748$ \\
\hline CALIFORNIA DAVIS & GLM+ & $2,297,876$ & $4,597,531$ & 962,950 & 254,872 & $8,113,229$ & 153,388 \\
\hline CALIFORNIA IRVINE & bGM+ & $2,739,752$ & $6,474,353$ & 294,234 & $\mathrm{UA} / \mathrm{NA}$ & $9,508,339$ & 65,401 \\
\hline CALIFORNIA LOS ANGELES & LM+ & $6,516,528$ & $8,021,917$ & UA/NA & 462,101 & $15,000,546$ & 418,452 \\
\hline CALIFORNIA RIVERSIDE & G+ & $1,282,122$ & $4,338,335$ & 56,016 & 69,405 & $5,745,878$ & 181,812 \\
\hline CALIFORNIA SAN DIEGO & bGM+ & $2,668,605$ & $6,520,966$ & 394,191 & 0 & $9,583,762$ & 264,931 \\
\hline CALIFORNIA SANTA BARBARA & G+ & $1,836,596$ & $3,962,387$ & 0 & 0 & $5,798,983$ & 152,549 \\
\hline CASE WESTERN RESERVE & GLM+ & 719,638 & $5,626,072$ & 219,293 & 137,605 & $6,702,608$ & 69,453 \\
\hline CHICAGO & bGLM+ & $6,144,425$ & $11,559,713$ & 0 & 0 & $17,704,138$ & 401,626 \\
\hline CINCINNATI & bGLM+ & $2,451,165$ & $7,075,210$ & 606,555 & 150,755 & $10,283,685$ & 134,264 \\
\hline COLORADO & bGL+ & $2,171,442$ & $7,158,550$ & 891,264 & 793,505 & $11,014,761$ & 108,434 \\
\hline COLORADO STATE & G+ & $1,322,568$ & $4,611,644$ & 263,633 & 348,119 & $6,545,964$ & 44,351 \\
\hline COLUMBIA & GLM+ & $6,098,651$ & $14,110,787$ & $2,084,407$ & $1,391,211$ & $23,685,056$ & 635,352 \\
\hline CONNECTICUT & BGLM+ & 956,188 & $6,958,737$ & 249,772 & 819,366 & $8,984,063$ & 165,115 \\
\hline CORNELL & BGL+ & $6,761,733$ & $7,934,603$ & 857,024 & 289,887 & $15,843,247$ & 158,581 \\
\hline DARTMOUTH & GM+ & $1,681,053$ & $7,088,387$ & 101,739 & 0 & $8,871,179$ & 117,408 \\
\hline DELAWARE & G+ & $2,217,605$ & $5,778,112$ & 544,957 & 268,414 & $8,809,088$ & 42,469 \\
\hline DUKE & bLM+ & $5,413,023$ & $9,865,125$ & 74,168 & $1,155,318$ & $16,507,634$ & 197,108 \\
\hline EMORY & BGLM+ & $5,908,477$ & $4,877,256$ & $3,639,948$ & 476,677 & $14,902,358$ & 133,881 \\
\hline FLORIDA & BbGLM+ & $1,348,788$ & $9,938,022$ & $1,034,154$ & 106,786 & $12,427,750$ & 120,869 \\
\hline FLORIDA STATE & BbLM+ & $1,287,909$ & $5,337,897$ & $1,297,356$ & 260,304 & $8,183,466$ & 45,618 \\
\hline GEORGE WASHINGTON & GLM+ & $2,634,298$ & $6,103,662$ & 273,006 & $1,377,163$ & $10,388,129$ & 145,442 \\
\hline GEORGETOWN & bGLM+ & $2,467,328$ & $7,843,448$ & 450,267 & 481,743 & $11,242,786$ & 108,293 \\
\hline GEORGIA & GL+ & $2,405,117$ & $8,415,725$ & 401,668 & 382,979 & $11,605,489$ & 254,042 \\
\hline GEORGIA TECH & G+ & 571,817 & $4,227,563$ & $1,406,161$ & 0 & $6,205,541$ & 38,418 \\
\hline GUELPH & BG+ & $1,418,595$ & $3,586,461$ & 138,475 & 540,877 & $5,684,408$ & 34,312 \\
\hline HARVARD & GLM+ & $13,455,145$ & $9,248,116$ & $11,532,084$ & 55,984 & $34,291,329$ & $1,111,119$ \\
\hline HAWAII & GLM+ & $1,375,014$ & $5,630,515$ & 117,630 & 42,641 & $7,165,800$ & 143,008 \\
\hline HOUSTON & bL+ & $1,760,006$ & $6,275,219$ & 394,690 & 481,962 & $8,911,877$ & 131,353 \\
\hline
\end{tabular}




\section{EXPENDITURES}

Salaries \& Wages Salaries \& Wages Salaries \& Wages Total Salaries Other Operating Total Library

Professional Staff Support Staff Student Assistants

\&Wages
Expenditures
Expenditures
(17)
(19)
(17.a)
(17.b)
(17.c)
(20) (Survey Question Number)

\begin{tabular}{|c|c|c|c|c|c|c|}
\hline $4,103,185$ & $2,112,002$ & 599,463 & $6,814,650$ & $2,052,276$ & $16,623,179$ & ALABAMA \\
\hline $7,206,687$ & $10,163,830$ & $1,114,997$ & $18,485,514$ & $11,174,043$ & $46,622,403$ & ALBERTA \\
\hline $5,763,875$ & $5,572,208$ & $1,111,218$ & $12,447,301$ & $6,217,440$ & $31,355,788$ & ARIZONA \\
\hline $5,303,529$ & $6,336,349$ & 625,979 & $12,265,857$ & $3,505,023$ & $27,342,037$ & ARIZONA STATE \\
\hline $2,843,289$ & $1,329,794$ & 338,558 & $4,511,641$ & $1,552,088$ & $11,741,607$ & AUBURN \\
\hline $5,202,930$ & $3,325,562$ & 642,093 & $9,170,585$ & $2,208,384$ & $21,007,389$ & BOSTON \\
\hline $5,370,271$ & $2,864,922$ & 766,067 & $9,001,260$ & $1,250,914$ & $19,328,235$ & BOSTON COLLEGE \\
\hline $7,461,653$ & $1,780,355$ & $2,883,577$ & $12,125,585$ & $3,616,792$ & $27,167,004$ & BRIGHAM YOUNG \\
\hline $8,499,949$ & $6,672,144$ & 834,442 & $16,006,535$ & $3,955,552$ & $35,014,948$ & BRITISH COLUMBIA \\
\hline $4,625,791$ & $4,081,895$ & 454,305 & $9,161,991$ & $2,126,374$ & $19,861,584$ & BROWN \\
\hline $17,244,826$ & $8,478,887$ & $3,099,034$ & $28,822,747$ & $6,674,045$ & $56,670,387$ & CALIFORNIA BERKELEY \\
\hline $4,178,107$ & $5,160,053$ & $1,105,796$ & $10,443,956$ & $1,677,155$ & $20,387,728$ & CALIFORNIA DAVIS \\
\hline $4,441,990$ & $4,982,519$ & 658,888 & $10,083,397$ & $2,702,701$ & $22,359,838$ & CALIFORNIA IRVINE \\
\hline $12,599,849$ & $12,612,217$ & $2,985,402$ & $28,197,468$ & $9,537,404$ & $53,153,870$ & CALIFORNIA LOS ANGELES \\
\hline $3,680,131$ & $3,605,831$ & 537,247 & $7,823,209$ & $1,378,904$ & $15,129,803$ & CALIFORNIA RIVERSIDE \\
\hline $7,262,657$ & $7,569,412$ & $1,498,369$ & $16,330,438$ & $4,282,331$ & $30,461,462$ & CALIFORNIA SAN DIEGO \\
\hline $3,167,866$ & $4,568,779$ & $1,103,902$ & $8,840,547$ & $5,435,713$ & $20,227,792$ & CALIFORNIA SANTA BARBARA \\
\hline $3,578,878$ & $1,444,997$ & 541,357 & $5,565,232$ & $1,064,181$ & $13,401,474$ & CASE WESTERN RESERVE \\
\hline $4,862,576$ & $6,440,066$ & $1,335,028$ & $12,637,670$ & $3,841,968$ & $34,585,402$ & CHICAGO \\
\hline $5,360,452$ & $1,937,400$ & 706,485 & $8,004,337$ & $1,783,625$ & $20,205,911$ & CINCINNATI \\
\hline $3,260,358$ & $4,605,715$ & 826,732 & $8,692,805$ & $1,521,928$ & $21,337,928$ & COLORADO \\
\hline $3,270,077$ & $2,182,892$ & 621,199 & $6,074,168$ & $2,523,449$ & $15,187,932$ & COLORADO STATE \\
\hline $20,103,853$ & $8,150,610$ & $2,400,405$ & $30,654,868$ & $6,974,601$ & $61,949,877$ & COLUMBIA \\
\hline $8,460,309$ & $5,387,214$ & 904,232 & $14,751,755$ & $2,198,984$ & $26,099,917$ & CONNECTICUT \\
\hline $8,228,884$ & $13,438,277$ & 999,647 & $22,666,808$ & $8,128,897$ & $46,797,533$ & CORNELL \\
\hline $4,132,674$ & $3,868,597$ & 439,344 & $8,440,615$ & $1,337,837$ & $18,767,039$ & DARTMOUTH \\
\hline $4,211,405$ & $3,226,144$ & 386,782 & $7,824,331$ & $1,802,698$ & $18,478,586$ & DELAWARE \\
\hline $10,833,246$ & $5,027,592$ & 583,129 & $16,443,967$ & $4,181,879$ & $37,330,588$ & DUKE \\
\hline $7,546,934$ & $4,635,575$ & 785,320 & $12,967,829$ & $3,932,346$ & $31,936,414$ & EMORY \\
\hline $6,042,307$ & $7,352,553$ & 558,923 & $13,953,783$ & $2,070,900$ & $28,573,302$ & FLORIDA \\
\hline $3,622,653$ & $2,864,197$ & 602,718 & $7,089,568$ & 644,076 & $15,962,728$ & FLORIDA STATE \\
\hline $5,513,970$ & $3,703,453$ & 671,347 & $9,888,770$ & $3,068,084$ & $23,490,425$ & GEORGE WASHINGTON \\
\hline $6,453,342$ & $4,518,308$ & 769,502 & $11,741,152$ & $2,820,784$ & $25,913,015$ & GEORGETOWN \\
\hline $4,051,061$ & $5,313,416$ & 741,766 & $10,106,243$ & $2,485,368$ & $24,451,142$ & GEORGIA \\
\hline $2,682,678$ & $2,303,715$ & 136,131 & $5,122,524$ & $1,066,271$ & $12,432,754$ & GEORGIA TECH \\
\hline $3,136,455$ & $3,085,513$ & 331,867 & $6,553,835$ & $1,157,100$ & $13,429,656$ & GUELPH \\
\hline $40,369,569$ & $20,660,430$ & $1,768,141$ & $62,798,140$ & $19,683,709$ & $117,884,297$ & HARVARD \\
\hline $5,563,594$ & $2,552,425$ & 971,620 & $9,087,639$ & $1,293,683$ & $17,690,130$ & HAWAII \\
\hline $2,795,072$ & $2,352,860$ & 484,005 & $5,631,937$ & $4,611,134$ & $19,286,301$ & HOUSTON \\
\hline
\end{tabular}




\section{EXPENDITURES}

Monographs Current Serials Other Library Miscellaneous Total Library Materials Materials Materials

\section{INSTITUTION}

\begin{tabular}{|c|c|c|c|c|c|c|c|}
\hline HOWARD & bLM+ & 246,474 & $3,360,936$ & 166,322 & 50,000 & $3,823,732$ & 63,000 \\
\hline ILLINOIS CHICAGO & $\mathrm{M}+$ & $1,292,036$ & $4,232,443$ & $3,341,282$ & 262,735 & $9,128,496$ & UA/NA \\
\hline ILLINOIS URBANA & bGL+ & $2,895,921$ & $9,626,380$ & 849,572 & 693,789 & $14,065,662$ & 285,420 \\
\hline INDIANA & GLM+ & $6,427,271$ & $8,782,161$ & $1,090,405$ & 205,029 & $16,504,866$ & 218,860 \\
\hline IOWA & bLM+ & $2,352,587$ & $9,918,930$ & 615,896 & 703,459 & $13,590,872$ & 151,742 \\
\hline IOWA STATE & G+ & $1,719,386$ & $7,105,893$ & 129,270 & 404,778 & $9,359,327$ & 127,105 \\
\hline JOHNS HOPKINS & $\mathrm{BbG}+$ & $2,030,703$ & $10,842,794$ & 878,413 & $1,072,306$ & $14,824,216$ & 77,266 \\
\hline KANSAS & BbLM+ & $2,084,991$ & $5,860,695$ & $1,055,099$ & 581,065 & $9,581,850$ & 135,168 \\
\hline KENT STATE & $\mathrm{BbG}+$ & 810,787 & $3,232,164$ & $\mathrm{UA} / \mathrm{NA}$ & 0 & $4,042,951$ & 77,038 \\
\hline KENTUCKY & GLM+ & $1,624,478$ & $6,421,219$ & $1,686,050$ & 684,330 & $10,416,077$ & 144,418 \\
\hline LAVAL & G+ & $2,706,726$ & $7,322,707$ & 155,102 & 190,331 & $10,374,866$ & 76,254 \\
\hline LOUISIANA STATE & GLM+ & $1,993,211$ & $5,283,693$ & 109,975 & 0 & $7,386,879$ & 49,292 \\
\hline LOUISVILLE & $\mathrm{BbG}+$ & 951,788 & $4,811,709$ & $3,442,528$ & 0 & $9,206,025$ & 107,458 \\
\hline MCGILL & BbGLM+ & $5,210,714$ & $10,187,365$ & 245,315 & 442,234 & $16,085,628$ & 246,275 \\
\hline MCMASTER & GM+ & $1,058,438$ & $5,714,150$ & 517,841 & 971,509 & $8,261,937$ & 4,067 \\
\hline MANITOBA & G+ & 2,037,195 & $5,822,024$ & 45,291 & 426,280 & $8,330,789$ & 160,107 \\
\hline MARYLAND & G & $1,588,899$ & $7,651,874$ & 638,088 & 218,746 & $10,097,607$ & 113,804 \\
\hline MASSACHUSETTS & G+ & $1,084,359$ & $5,028,032$ & 403,355 & 29,273 & $6,545,019$ & 97,816 \\
\hline MIT & G+ & $1,624,976$ & $6,817,728$ & 371,925 & 71,926 & $8,886,555$ & 197,119 \\
\hline MIAMI & bGLM+ & $3,147,172$ & $9,739,138$ & 0 & 327,278 & $13,213,588$ & 91,131 \\
\hline MICHIGAN & GLM+ & $5,186,157$ & $13,324,502$ & 171,764 & $1,843,453$ & $20,525,876$ & 437,616 \\
\hline MICHIGAN STATE & GL+ & $2,244,634$ & $8,444,193$ & 438,791 & 139,686 & $11,267,304$ & 202,351 \\
\hline MINNESOTA & bGLM+ & $3,436,528$ & $11,501,644$ & $1,362,691$ & 277,421 & $16,578,284$ & 297,751 \\
\hline MISSOURI & GL+ & $1,029,157$ & $5,985,414$ & $1,438,636$ & 62,373 & $8,515,580$ & 102,702 \\
\hline MONTREAL & GLM+ & $1,976,784$ & $9,211,831$ & 95,919 & 10,271 & $11,294,805$ & 140,224 \\
\hline NEBRASKA & GL+ & 427,296 & $6,401,031$ & 155,183 & 56,478 & $7,039,988$ & 144,081 \\
\hline NEW MEXICO & GLM+ & $1,377,722$ & $6,581,497$ & 269,897 & 447,723 & $8,676,839$ & 83,212 \\
\hline NEW YORK & bGLM+ & $4,017,073$ & $11,165,164$ & $1,129,396$ & 186,903 & $16,498,536$ & 470,701 \\
\hline NORTH CAROLINA & GLM+ & $3,158,630$ & $9,443,093$ & $3,614,174$ & 106,676 & $16,322,573$ & 265,396 \\
\hline NORTH CAROLINA STATE & G+ & $1,862,181$ & $6,616,791$ & 415,264 & 914,842 & $9,809,078$ & 210,919 \\
\hline NORTHWESTERN & BGLM+ & $3,631,003$ & $8,783,955$ & 688,964 & 493,004 & $13,596,926$ & 174,615 \\
\hline NOTRE DAME & bGL+ & $4,979,282$ & $6,573,240$ & 0 & 53,915 & $11,606,437$ & 103,076 \\
\hline OHIO & $\mathrm{BbG}+$ & $1,277,551$ & $3,642,738$ & 520,206 & 200,514 & $5,641,009$ & 38,319 \\
\hline OHIO STATE & bGLM+ & $3,232,837$ & $8,765,104$ & 595,835 & 585,062 & $13,178,838$ & 322,443 \\
\hline OKLAHOMA & GLM+ & $3,313,871$ & $8,582,861$ & $1,803,987$ & 381,114 & $14,081,833$ & 218,547 \\
\hline OKLAHOMA STATE & BGM+ & $1,402,560$ & $5,286,432$ & 113,866 & 38,453 & $6,841,311$ & 69,145 \\
\hline OREGON & bGL+ & $1,675,671$ & $3,976,853$ & $\mathrm{UA} / \mathrm{NA}$ & $1,065,756$ & $6,718,280$ & 106,229 \\
\hline PENNSYLVANIA & GLM+ & $3,734,557$ & $10,090,093$ & 564,886 & 779,323 & $15,168,859$ & 317,016 \\
\hline PENNSYLVANIA STATE & BGLM+ & $3,946,715$ & $12,954,911$ & 786,018 & 138,479 & $17,826,123$ & 402,103 \\
\hline
\end{tabular}




\section{EXPENDITURES}

Salaries \& Wages Salaries \& Wages Salaries \& Wages Total Salaries Other Operating Total Library

Professional Staff Support Staff Student Assistants

\&Wages
Expenditures
Expenditures
(17)
(19)
(17.a)
(17.b)
(17.c)
(20) (Survey Question Number)

\begin{tabular}{|c|c|c|c|c|c|c|}
\hline $2,893,992$ & $2,138,628$ & 402,691 & $5,435,311$ & 951,272 & $10,273,315$ & HOWARD \\
\hline $3,717,050$ & $3,712,395$ & 444,038 & $7,873,483$ & $2,256,901$ & $19,258,880$ & ILLINOIS CHICAGO \\
\hline $11,550,518$ & $7,630,897$ & $1,830,047$ & $21,011,462$ & $4,351,948$ & $39,714,492$ & ILLINOIS URBANA \\
\hline $10,068,396$ & $5,214,735$ & $1,881,482$ & $17,164,613$ & $5,129,811$ & $39,018,150$ & INDIANA \\
\hline $6,140,434$ & $5,232,797$ & 962,134 & $12,335,365$ & $1,542,269$ & $27,620,248$ & IOWA \\
\hline $3,152,049$ & $3,677,430$ & 454,319 & $7,283,798$ & $1,724,165$ & $18,494,395$ & IOWA STATE \\
\hline $5,657,282$ & $4,671,961$ & 193,813 & $10,523,056$ & $3,726,050$ & $29,150,588$ & JOHNS HOPKINS \\
\hline $5,809,922$ & $3,492,891$ & 996,691 & $10,299,504$ & $2,415,971$ & $22,432,493$ & KANSAS \\
\hline $3,053,683$ & $1,279,398$ & 471,897 & $4,804,978$ & $2,958,811$ & $11,883,778$ & KENT STATE \\
\hline $4,909,905$ & $2,668,457$ & 678,531 & $8,256,893$ & $2,597,096$ & $21,414,484$ & KENTUCKY \\
\hline $4,305,000$ & $5,855,749$ & 48,338 & $10,209,086$ & 607,324 & $21,267,530$ & LAVAL \\
\hline $3,252,026$ & $2,047,136$ & 498,839 & $5,798,001$ & $1,341,854$ & $14,576,026$ & LOUISIANA STATE \\
\hline $3,087,778$ & $2,702,904$ & 485,063 & $6,275,745$ & $3,564,899$ & $19,154,127$ & LOUISVILLE \\
\hline $6,034,641$ & $7,639,133$ & 821,413 & $14,495,187$ & $4,316,278$ & $35,143,367$ & MCGILL \\
\hline $3,011,223$ & $4,123,876$ & 603,919 & $7,739,018$ & $1,404,406$ & $17,409,428$ & MCMASTER \\
\hline $5,336,296$ & $5,705,143$ & 711,923 & $11,753,362$ & $1,799,754$ & $22,044,012$ & MANITOBA \\
\hline $8,425,628$ & $3,461,393$ & 712,594 & $12,599,615$ & $1,937,553$ & $24,748,579$ & MARYLAND \\
\hline $3,874,503$ & $3,207,608$ & 529,560 & $7,611,671$ & $1,741,283$ & $15,995,789$ & MASSACHUSETTS \\
\hline $8,322,977$ & $4,009,913$ & 353,627 & $12,686,517$ & $3,276,441$ & $25,046,632$ & MIT \\
\hline $4,758,964$ & $4,174,269$ & 708,373 & $9,641,606$ & $4,304,519$ & $27,250,844$ & MIAMI \\
\hline $11,897,358$ & $12,161,191$ & $1,794,610$ & $25,853,159$ & $4,782,459$ & $51,599,110$ & MICHIGAN \\
\hline $5,251,600$ & $5,019,324$ & $1,398,441$ & $11,669,365$ & $2,467,965$ & $25,606,985$ & MICHIGAN STATE \\
\hline $7,854,878$ & $8,745,597$ & $1,517,220$ & $18,117,695$ & $5,740,315$ & $40,734,045$ & MINNESOTA \\
\hline $3,511,413$ & $2,454,935$ & 419,305 & $6,385,653$ & $1,690,135$ & $16,694,070$ & MISSOURI \\
\hline $7,162,773$ & $10,772,910$ & UA/NA & $17,935,683$ & $1,205,676$ & $30,576,387$ & MONTREAL \\
\hline $3,433,931$ & $2,961,885$ & 654,997 & $7,050,813$ & $1,382,252$ & $15,617,134$ & NEBRASKA \\
\hline $4,863,734$ & $6,423,517$ & 717,864 & $12,005,115$ & $3,319,369$ & $24,084,535$ & NEW MEXICO \\
\hline $9,617,319$ & $9,526,571$ & $1,559,454$ & $20,703,344$ & $6,930,149$ & $44,602,730$ & NEW YORK \\
\hline $10,686,200$ & $7,114,097$ & $1,143,426$ & $18,943,723$ & $5,591,823$ & $41,123,515$ & NORTH CAROLINA \\
\hline $7,228,469$ & $3,087,654$ & 643,498 & $10,959,621$ & $7,270,144$ & $28,249,762$ & NORTH CAROLINA STATE \\
\hline $7,129,458$ & $4,684,651$ & 703,498 & $12,517,607$ & $2,857,993$ & $29,147,141$ & NORTHWESTERN \\
\hline $4,893,032$ & $4,920,009$ & 493,386 & $10,306,427$ & $2,061,290$ & $24,077,230$ & NOTRE DAME \\
\hline $2,235,553$ & $2,576,608$ & 665,623 & $5,477,784$ & $1,233,362$ & $12,390,474$ & $\mathrm{OHIO}$ \\
\hline $8,394,752$ & $5,597,577$ & $2,650,050$ & $16,642,379$ & $8,329,578$ & $38,473,238$ & OHIO STATE \\
\hline $3,179,813$ & $2,405,624$ & $1,065,528$ & $6,650,965$ & $1,912,562$ & $22,863,907$ & OKLAHOMA \\
\hline $4,490,310$ & $2,766,159$ & 612,148 & $7,868,617$ & $2,163,237$ & $16,942,310$ & OKLAHOMA STATE \\
\hline $3,741,606$ & $2,888,147$ & $1,049,169$ & $7,678,922$ & $1,973,830$ & $16,477,261$ & OREGON \\
\hline $8,046,266$ & $8,094,502$ & 850,456 & $16,991,224$ & $5,943,884$ & $38,011,711$ & PENNSYLVANIA \\
\hline $10,507,239$ & $13,203,238$ & 726,145 & $24,436,622$ & $5,021,538$ & $47,686,386$ & PENNSYLVANIA STATE \\
\hline
\end{tabular}




\section{EXPENDITURES}

Monographs Current Serials Other Library Miscellaneous Total Library Materials Materials Materials

INSTITUTION

\begin{tabular}{|c|c|c|c|c|c|c|c|}
\hline PITTSBURGH & BGLM+ & $2,785,410$ & $9,243,253$ & 339,178 & $2,489,183$ & $14,857,024$ & 195,737 \\
\hline PRINCETON & bG+ & $7,721,342$ & $10,769,945$ & $2,113,814$ & 592,003 & $21,197,104$ & 513,804 \\
\hline PURDUE & G+ & $1,447,270$ & $7,022,039$ & $1,934,647$ & 176,264 & $10,580,220$ & 89,563 \\
\hline QUEENS & $\mathrm{LM}+$ & $1,630,058$ & $7,367,618$ & 181,464 & 364,153 & $9,543,294$ & 85,513 \\
\hline RICE & G+ & $4,210,131$ & $6,139,862$ & 372,956 & 405,094 & $11,128,043$ & 50,080 \\
\hline ROCHESTER & $\mathrm{bM}+$ & $1,161,236$ & $6,258,440$ & 358,573 & 31,333 & $7,809,582$ & 111,793 \\
\hline RUTGERS & BGL+ & $1,352,783$ & $7,774,275$ & 962,985 & 364,601 & $10,454,644$ & 111,749 \\
\hline SASKATCHEWAN & GLM+ & $2,130,561$ & $6,347,299$ & $\mathrm{UA} / \mathrm{NA}$ & 464,305 & $8,942,165$ & 72,758 \\
\hline SOUTH CAROLINA & $\mathrm{LM}+$ & $1,357,022$ & $6,116,438$ & 411,847 & 166,497 & $8,051,804$ & 72,821 \\
\hline SOUTHERN CALIFORNIA & BGLM+ & $2,874,283$ & $7,878,187$ & 138,947 & 241,037 & $11,132,454$ & 157,736 \\
\hline SOUTHERN ILLINOIS & $\mathrm{LM}+$ & 736,010 & $5,866,259$ & 152,081 & 276,904 & $7,031,254$ & 125,101 \\
\hline SUNY-ALBANY & G+ & 885,746 & $4,215,691$ & 47,580 & 255,784 & $5,404,801$ & 56,502 \\
\hline SUNY-BUFFALO & GLM+ & $1,273,433$ & $7,172,458$ & 148,791 & 59,272 & $8,653,954$ & 67,779 \\
\hline SUNY-STONY BROOK & $\mathrm{M}+$ & 420,700 & $5,221,576$ & 397,263 & $1,071,596$ & $7,111,135$ & 60,579 \\
\hline SYRACUSE & GL+ & $1,258,269$ & $5,316,483$ & 463,151 & 0 & $7,037,903$ & 51,230 \\
\hline TEMPLE & BbGLM+ & $2,216,053$ & $5,690,849$ & $3,688,587$ & 7,461 & $11,602,950$ & 141,357 \\
\hline TENNESSEE & BbGLM+ & $2,132,077$ & $9,432,867$ & 455,852 & 189,224 & $12,210,020$ & 103,438 \\
\hline TEXAS & GL+ & $3,349,386$ & $9,820,242$ & $4,186,655$ & 259,408 & $17,615,691$ & 202,482 \\
\hline TEXAS A\&M & $\mathrm{BbG}+$ & $5,180,137$ & $11,101,887$ & 720,576 & 0 & $17,002,600$ & 113,146 \\
\hline TEXAS TECH & BLM+ & $2,707,128$ & $7,318,882$ & 229,414 & 824,265 & $11,079,689$ & 92,165 \\
\hline TORONTO & $\mathrm{B}+$ & $8,349,661$ & $11,493,126$ & $5,979,621$ & UA/NA & $25,822,409$ & 315,787 \\
\hline TULANE & BLM+ & $1,770,102$ & $6,093,411$ & 323,048 & 2,000 & $8,188,561$ & 45,092 \\
\hline UTAH & LM & $1,924,324$ & $5,739,547$ & 60,902 & 255,769 & $7,980,542$ & 114,166 \\
\hline VANDERBILT & LM+ & $1,747,797$ & $9,514,270$ & 133,925 & 39,800 & $11,435,792$ & 127,912 \\
\hline VIRGINIA & GLM+ & $3,117,556$ & $8,426,644$ & $1,523,169$ & 400,283 & $13,467,652$ & 133,378 \\
\hline VIRGINIA TECH & G & $1,126,563$ & $5,423,174$ & $\mathrm{UA} / \mathrm{NA}$ & 493,307 & $7,043,044$ & 50,749 \\
\hline WASHINGTON & GLM+ & $3,085,456$ & $11,393,157$ & 268,939 & 114,875 & $14,862,427$ & 213,119 \\
\hline WASHINGTON STATE & BG+ & $1,162,960$ & $5,042,750$ & 8,215 & 210,551 & $6,424,476$ & 45,761 \\
\hline WASHINGTON U.-ST. LOUIS & bGLM+ & $1,695,048$ & $8,486,004$ & $2,062,167$ & 219,513 & $12,462,732$ & 109,437 \\
\hline WATERLOO & G+ & $1,267,256$ & $4,649,880$ & 122,520 & 494,105 & $6,533,760$ & 49,765 \\
\hline WAYNE STATE & + & $1,181,664$ & $7,495,718$ & 0 & 329,760 & $9,007,142$ & 79,889 \\
\hline WESTERN ONTARIO & GL+ & $2,266,855$ & $9,129,335$ & UA/NA & 241,691 & $11,637,880$ & 116,165 \\
\hline WISCONSIN & GLM+ & $2,017,313$ & $6,263,640$ & $2,252,086$ & 441,493 & $10,974,532$ & 184,979 \\
\hline YALE & LM+ & $11,741,599$ & $5,557,697$ & $16,045,965$ & 0 & $33,345,261$ & 515,555 \\
\hline YORK & BGL+ & $2,577,912$ & $7,881,161$ & 209,117 & 103,349 & $10,771,539$ & 177,918 \\
\hline BOSTON PUBLIC & G & $3,754,079$ & $1,150,588$ & $1,152,171$ & 37,490 & $6,094,328$ & 10,614 \\
\hline CISTI & + & 518,789 & $10,578,513$ & 301,657 & 11,692 & $11,410,651$ & 0 \\
\hline CENTER FOR RESEARCH LIBRARIES & + & 78,924 & 272,539 & 655,576 & 20,421 & $1,027,460$ & UA/NA \\
\hline LIBRARY OF CONGRESS & bG+ & $5,202,000$ & $6,395,000$ & $6,802,984$ & 260,854 & $18,660,838$ & $1,352,050$ \\
\hline
\end{tabular}




\section{EXPENDITURES}

Salaries \& Wages Salaries \& Wages Salaries \& Wages Total Salaries Other Operating Total Library

Professional Staff Support Staff Student Assistants

\&Wages
Expenditures
Expenditures
(17)
(19)
(17.a)
(17.b)
(17.c)
(20) (Survey Question Number)

\begin{tabular}{|c|c|c|c|c|c|c|}
\hline $6,678,904$ & $4,906,078$ & 953,840 & $12,538,822$ & $4,068,526$ & $31,660,109$ & PITTSBURGH \\
\hline $9,541,754$ & $8,347,687$ & 899,642 & $18,789,083$ & $4,919,618$ & $45,419,609$ & PRINCETON \\
\hline $5,891,096$ & $4,214,507$ & 938,232 & $11,043,835$ & $3,860,300$ & $25,573,918$ & PURDUE \\
\hline $3,414,591$ & $4,687,169$ & 315,377 & $8,417,136$ & $1,502,065$ & $19,548,008$ & QUEENS \\
\hline $3,442,262$ & $1,536,384$ & 167,747 & $5,146,393$ & 714,789 & $17,039,305$ & RICE \\
\hline $6,349,979$ & $2,155,275$ & 513,936 & $9,019,190$ & $6,361,120$ & $23,301,685$ & ROCHESTER \\
\hline $7,325,228$ & $10,986,436$ & $1,213,008$ & $19,524,672$ & $2,342,995$ & $32,434,060$ & RUTGERS \\
\hline $4,139,164$ & $3,222,172$ & 276,594 & $7,637,931$ & $1,248,714$ & $17,901,568$ & SASKATCHEWAN \\
\hline $4,058,321$ & $3,317,677$ & 598,802 & $7,974,800$ & $3,643,160$ & $19,742,585$ & SOUTH CAROLINA \\
\hline $9,015,433$ & $6,165,255$ & $1,968,628$ & $17,149,316$ & $9,953,703$ & $38,393,209$ & SOUTHERN CALIFORNIA \\
\hline $2,847,194$ & $2,860,590$ & 990,270 & $6,698,054$ & $1,443,490$ & $15,297,899$ & SOUTHERN ILLINOIS \\
\hline $3,946,577$ & $2,204,293$ & 495,310 & $6,646,180$ & 75,884 & $12,183,367$ & SUNY-ALBANY \\
\hline $6,723,181$ & $2,485,850$ & $1,289,276$ & $10,498,307$ & $1,567,889$ & $20,787,929$ & SUNY-BUFFALO \\
\hline $5,064,220$ & 902,657 & 423,382 & $6,390,259$ & 749,058 & $14,311,031$ & SUNY-STONY BROOK \\
\hline $4,039,921$ & $3,971,391$ & 478,594 & $8,489,906$ & $1,549,946$ & $17,128,985$ & SYRACUSE \\
\hline $4,806,835$ & $2,440,474$ & 693,279 & $7,940,588$ & $3,109,273$ & $22,794,168$ & TEMPLE \\
\hline $5,479,556$ & $3,696,339$ & 728,009 & $9,903,904$ & $1,338,868$ & $23,556,230$ & TENNESSEE \\
\hline $8,641,986$ & $10,672,107$ & $1,458,561$ & $20,772,654$ & $5,259,179$ & $43,850,006$ & TEXAS \\
\hline $7,814,173$ & $3,612,683$ & 901,975 & $12,328,831$ & $4,705,074$ & $34,149,651$ & TEXAS A\&M \\
\hline $4,929,494$ & $3,343,608$ & $1,242,656$ & $9,515,758$ & $4,497,118$ & $25,184,730$ & TEXAS TECH \\
\hline $15,348,537$ & $16,308,262$ & $4,004,668$ & $35,661,466$ & $6,354,878$ & $68,154,539$ & TORONTO \\
\hline $2,899,493$ & $2,040,338$ & 255,212 & $5,195,043$ & 748,643 & $14,177,339$ & TULANE \\
\hline $5,281,736$ & $5,981,915$ & $1,613,307$ & $12,876,958$ & $3,724,178$ & $24,695,844$ & UTAH \\
\hline $6,434,470$ & $3,273,212$ & 451,967 & $10,159,649$ & $3,004,230$ & $24,727,583$ & VANDERBILT \\
\hline $7,009,219$ & $9,063,042$ & 848,972 & $16,921,233$ & $5,407,540$ & $35,929,803$ & VIRGINIA \\
\hline $2,342,125$ & $2,866,591$ & 406,992 & $5,615,708$ & $1,010,566$ & $13,720,067$ & VIRGINIA TECH \\
\hline $11,904,128$ & $7,798,716$ & $2,327,126$ & $22,029,970$ & $3,749,314$ & $40,854,830$ & WASHINGTON \\
\hline $3,022,487$ & $3,070,487$ & 629,485 & $6,722,459$ & $1,517,246$ & $14,709,942$ & WASHINGTON STATE \\
\hline $6,014,991$ & $3,486,702$ & 717,370 & $10,219,063$ & $4,556,365$ & $27,347,597$ & WASHINGTON U.-ST. LOUIS \\
\hline $2,657,553$ & $3,825,489$ & 669,729 & $7,152,770$ & $1,738,974$ & $15,475,270$ & WATERLOO \\
\hline $5,225,694$ & $2,967,890$ & $1,155,782$ & $9,349,366$ & $2,365,219$ & $20,801,616$ & WAYNE STATE \\
\hline $4,383,118$ & $3,945,402$ & 300,473 & $8,628,993$ & 934,680 & $21,317,718$ & WESTERN ONTARIO \\
\hline $14,155,552$ & $6,544,134$ & $2,759,744$ & $23,459,430$ & $8,260,282$ & $42,879,223$ & WISCONSIN \\
\hline $20,166,081$ & $14,403,246$ & $1,211,749$ & $35,781,076$ & $11,815,578$ & $81,457,470$ & YALE \\
\hline $5,774,020$ & $5,093,102$ & $1,065,130$ & $11,932,252$ & $3,779,397$ & $26,661,107$ & YORK \\
\hline $11,752,721$ & $11,996,048$ & 583,986 & $24,332,755$ & $12,724,880$ & $43,162,577$ & BOSTON PUBLIC \\
\hline $\mathrm{UA} / \mathrm{NA}$ & UA/NA & $\mathrm{UA} / \mathrm{NA}$ & $22,822,493$ & $11,734,582$ & $45,967,726$ & CISTI \\
\hline $1,696,015$ & 548,341 & 244,162 & $2,488,518$ & 742,819 & $4,258,798$ & CENTER FOR RESEARCH LIBRARIES \\
\hline $323,924,456$ & $\mathrm{UA} / \mathrm{NA}$ & $\mathrm{UA} / \mathrm{NA}$ & $323,924,456$ & $325,864,203$ & $669,801,547$ & LIBRARY OF CONGRESS \\
\hline
\end{tabular}




\section{EXPENDITURES}

Monographs Current Serials Other Library Miscellaneous Total Library Materials Materials Materials

\section{Notes}

\begin{tabular}{|c|c|c|c|c|c|c|c|}
\hline NATIONAL AGRICULTURAL LIBRARY & $\mathrm{bG}+$ & 219,032 & $3,057,505$ & 509,560 & 0 & $3,786,097$ & 2,500 \\
\hline LIBRARY \& ARCHIVES CANADA & G+ & 777,674 & 450,217 & 445,156 & 132,790 & $1,805,837$ & $\mathrm{UA} / \mathrm{NA}$ \\
\hline NATIONAL LIBRARY OF MEDICINE & G+ & $1,325,549$ & $7,689,804$ & 38,303 & & $9,053,656$ & 138,023 \\
\hline NEW YORK PUBLIC & + & $7,772,851$ & $3,595,074$ & $3,856,986$ & $\mathrm{UA} / \mathrm{NA}$ & $15,224,911$ & 668,904 \\
\hline NEW YORK STATE & G+ & $1,740,000$ & $2,530,000$ & $\mathrm{UA} / \mathrm{NA}$ & 0 & $4,270,000$ & 27,823 \\
\hline SMITHSONIAN & $\mathrm{G}^{+}$ & 405,964 & $1,102,830$ & 0 & 51,961 & $1,560,755$ & 32,048 \\
\hline
\end{tabular}

+ - See Footnotes

L - Includes Law library

G - Government documents not included in serials count B - Includes branch campuses

b - Basis of volume count is bibliographic
U/A - Unavailable

N/A - Not Applicable

. - no data supplied

\section{Summary Data}

Monographs Current Serials Other Library Miscellaneous Total Library Materials Materials Materials
Contract Binding

(Survey Question Number) (15.b)

(15.c) (15.d)

(15)
(16)

\section{UNIVERSITY LIBRARIES}

Mean
Median
High
Low
Totals
Number of Libraries Reportin

$2,716,677$
$2,037,195$
$13,455,145$
246,474
$306,984,547$

$7,369,145$
$7,075,210$
$14,110,787$
$3,232,164$
$832,713,383$
113

$1,023,252$
403,355

372,877

$11,421,020$

168,796

$16,045,965$

$2,489,183$

$10,388,129$

122,985

0

$109,487,911$

113

107

$41,389,384$

$34,291,329$

$1,111,119$

4,067

$18,905,174$

112

\section{NonUniVersity LibraRies}

\author{
Median \\ Totals \\ Number of Libraries Reporting
}

GRAND TOTALS

$\begin{array}{rr}1,051,612 & 2,793,753 \\ 21,794,863 & 36,822,070 \\ 10 & 10\end{array}$

509,560

$13,762,393$

9

$328,779,410$
$869,535,452$

$123,250,303$

$41,904,592 \quad 1,363,469,758$

$21,137,136$ 


\section{EXPENDITURES}

Salaries \& Wages Salaries \& Wages Salaries \& Wages Professional Staff
Total Salaries Other Operating \&Wages
Total Library

Expenditures

$$
\text { (17.a) }
$$$$
\text { (17.a) }
$$
(17)
(17.b)
(17.c)
(19)
(20) (Survey Question Number)

INSTITUTION

\begin{tabular}{rrrrrrr}
\hline $12,583,397$ & $2,860,836$ & 29,890 & $15,474,123$ & $9,540,548$ & $28,803,268$ & NATIONAL AGRICULTURAL LIBRARY \\
$52,622,170$ & $23,111,200$ & 704,893 & $76,438,263$ & UA/NA & $78,244,100$ & LIBRARY \& ARCHIVES CANADA \\
$21,288,580$ & $4,563,038$ & 527,492 & $26,379,110$ & $25,183,957$ & $60,754,746$ & NATIONAL LIBRARY OF MEDICINE \\
$16,973,850$ & $11,391,606$ & $1,469,329$ & $29,834,785$ & $12,537,654$ & $58,266,254$ & NEW YORK PUBLIC \\
UA/NA & UA/NA & UA/NA & $6,471,850$ & 938,122 & $11,707,795$ & NEW YORK STATE \\
$4,954,790$ & $2,069,238$ & 9,458 & $7,033,486$ & $1,196,254$ & $9,822,543$ & SMITHSONIAN
\end{tabular}

\section{Summary Data}

Salaries \& Wages Salaries \& Wages Salaries \& Wages Professional Staff Support Staff Student Assistants
Total Salaries Other Operating Total Library \&Wages Expenditures Expenditures (17.a)

(17.b)

(17.c)
(19)
(20) (Survey Question Number)

\begin{tabular}{|c|c|c|c|c|c|c|}
\hline & & & & & & UNIVERSITY LIBRARIES \\
\hline $6,471,628$ & $5,139,087$ & 940,571 & $12,542,962$ & $3,476,419$ & $27,607,703$ & Mean \\
\hline $5,303,529$ & $4,081,895$ & 714,982 & $10,209,086$ & $2,597,096$ & $23,556,230$ & Median \\
\hline $40,369,569$ & $20,660,430$ & $4,004,668$ & $62,798,140$ & $19,683,709$ & $117,884,297$ & High \\
\hline $2,235,553$ & 902,657 & 48,338 & $4,511,641$ & 75,884 & $10,273,315$ & Low \\
\hline $731,293,975$ & $580,716,776$ & $105,343,950$ & $1,417,354,701$ & $392,835,394$ & $3,119,670,494$ & Totals \\
\hline \multirow[t]{2}{*}{113} & 113 & 112 & 113 & 113 & 113 & Number of Libraries Reporting \\
\hline & & & & & & NONUNIVERSITY LibRARIES \\
\hline $14,778,624$ & $4,563,038$ & 527,492 & $23,577,624$ & $11,734,582$ & $44,565,151$ & Median \\
\hline $445,795,979$ & $56,540,307$ & $3,569,210$ & $535,199,838$ & $400,463,019$ & $1,010,789,353$ & Totals \\
\hline 8 & 7 & 7 & 10 & 9 & 10 & Number of Libraries Reporting \\
\hline $1,177,089,954$ & $637,257,083$ & $108,913,160$ & $1,952,554,540$ & $793,298,413$ & $4,130,459,847$ & GRAND TOTALS \\
\hline
\end{tabular}




\section{Electronic Resources Expenditures}

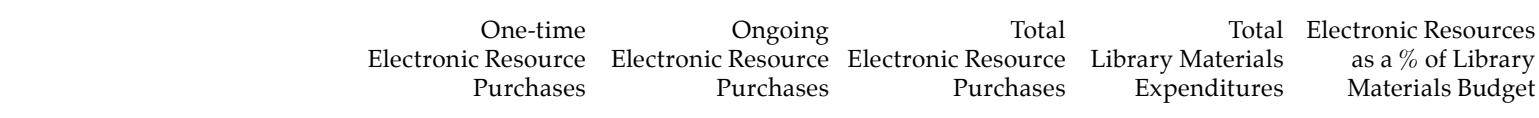

(Survey Question Number)

(21)

(22)

$(21+22)$

(15)

INSTITUTION

Notes

\begin{tabular}{|c|c|c|c|c|c|c|}
\hline ALABAMA & bGLM+ & 306,950 & $3,730,223$ & $4,037,173$ & $7,688,246$ & 52.51 \\
\hline ALBERTA & G+ & $7,356,162$ & $7,165,442$ & $14,521,604$ & $16,880,131$ & 86.03 \\
\hline ARIZONA & bGLM+ & $1,009,605$ & $6,336,444$ & $7,346,049$ & $12,627,388$ & 58.18 \\
\hline ARIZONA STATE & BGL+ & 68,793 & $5,997,836$ & $6,066,629$ & $11,433,551$ & 53.06 \\
\hline AUBURN & bG+ & 0 & $4,704,780$ & $4,704,780$ & $5,629,762$ & 83.57 \\
\hline BOSTON & $\mathrm{LM}+$ & 534,911 & $2,827,120$ & $3,362,031$ & $9,468,057$ & 35.51 \\
\hline BOSTON COLLEGE & $\mathrm{L}+$ & 638,363 & $4,170,504$ & $4,808,867$ & $8,979,309$ & 53.55 \\
\hline BRIGHAM YOUNG & $\mathrm{L}+$ & $\mathrm{UA} / \mathrm{NA}$ & $5,173,184$ & $5,173,184$ & $11,307,847$ & 45.75 \\
\hline BRITISH COLUMBIA & G+ & 176,642 & 7,471,946 & $7,648,588$ & $14,917,231$ & 51.27 \\
\hline BROWN & bG+ & 223,391 & $4,319,870$ & $4,543,261$ & $8,494,260$ & 53.49 \\
\hline CALIFORNIA BERKELEY & GL+ & 523,848 & $5,998,556$ & $6,522,404$ & $20,118,847$ & 32.42 \\
\hline CALIFORNIA DAVIS & GLM+ & 859,806 & $3,527,361$ & $4,387,167$ & $8,113,229$ & 54.07 \\
\hline CALIFORNIA IRVINE & bGM+ & 483,924 & $4,240,672$ & $4,724,596$ & $9,508,339$ & 49.69 \\
\hline CALIFORNIA LOS ANGELES & $\mathrm{LM}+$ & 339,502 & $4,522,530$ & $4,862,032$ & $15,000,546$ & 32.41 \\
\hline CALIFORNIA RIVERSIDE & G+ & 4,002 & $2,274,782$ & $2,278,784$ & $5,745,878$ & 39.66 \\
\hline CALIFORNIA SAN DIEGO & bGM+ & 474,626 & $4,809,297$ & $5,283,923$ & $9,583,762$ & 55.13 \\
\hline CALIFORNIA SANTA BARBARA & G+ & 16,195 & $2,680,741$ & $2,696,936$ & $5,798,983$ & 46.51 \\
\hline CASE WESTERN RESERVE & GLM+ & 142,721 & $3,374,918$ & $3,517,639$ & $6,702,608$ & 52.48 \\
\hline CHICAGO & bGLM+ & 872,864 & $4,894,830$ & $5,767,694$ & $17,704,138$ & 32.58 \\
\hline CINCINNATI & bGLM+ & 533,250 & $5,067,799$ & $5,601,049$ & $10,283,685$ & 54.47 \\
\hline COLORADO & bGL+ & 779,715 & $5,848,781$ & $6,628,496$ & $11,014,761$ & 60.18 \\
\hline COLORADO STATE & G+ & 324,857 & $3,867,699$ & $4,192,556$ & $6,545,964$ & 64.05 \\
\hline COLUMBIA & GLM+ & $1,712,309$ & $9,018,539$ & $10,730,848$ & $23,685,056$ & 45.31 \\
\hline CONNECTICUT & BGLM+ & 109,074 & $5,301,347$ & $5,410,421$ & $8,984,063$ & 60.22 \\
\hline CORNELL & BGL+ & 131,274 & $6,910,052$ & $7,041,326$ & $15,843,247$ & 44.44 \\
\hline DARTMOUTH & GM+ & 78,120 & $5,166,265$ & $5,244,385$ & $8,871,179$ & 59.12 \\
\hline DELAWARE & G+ & 463,689 & $5,085,956$ & $5,549,645$ & $8,809,088$ & 63.00 \\
\hline DUKE & bLM+ & $1,682,971$ & $6,318,112$ & $8,001,083$ & $16,507,634$ & 48.47 \\
\hline EMORY & BGLM+ & 199,506 & $4,313,531$ & $4,513,037$ & $14,902,358$ & 30.28 \\
\hline FLORIDA & BbGLM+ & 138,982 & $5,542,246$ & $5,681,228$ & $12,427,750$ & 45.71 \\
\hline FLORIDA STATE & BbLM+ & 64,944 & $5,241,256$ & $5,306,200$ & $8,183,466$ & 64.84 \\
\hline GEORGE WASHINGTON & GLM+ & 313,613 & $2,078,086$ & $2,391,699$ & $10,388,129$ & 23.02 \\
\hline GEORGETOWN & bGLM+ & 187,091 & $3,986,516$ & $4,173,607$ & $11,242,786$ & 37.12 \\
\hline GEORGIA & GL+ & 193,892 & $6,121,332$ & $6,315,224$ & $11,605,489$ & 54.42 \\
\hline GEORGIA TECH & G+ & 116,059 & $4,042,589$ & $4,158,648$ & $6,205,541$ & 67.02 \\
\hline GUELPH & $\mathrm{BG}+$ & 323,433 & $3,340,713$ & $3,664,146$ & $5,684,408$ & 64.46 \\
\hline HARVARD & GLM+ & $8,300,095$ & $\mathrm{UA} / \mathrm{NA}$ & $8,300,095$ & $34,291,329$ & 24.20 \\
\hline HAWAII & GLM+ & 5,550 & $3,493,135$ & $3,498,685$ & $7,165,800$ & 48.82 \\
\hline HOUSTON & $\mathrm{bL}+$ & 398,167 & $4,259,699$ & $4,657,866$ & $8,911,877$ & 52.27 \\
\hline
\end{tabular}




\section{Electronic Resources Expenditures}

Bibl. Utilities, Networks, etc. Bibl. Utilities, Networks, etc. Computer Hardware and Document Delivery/ External Expenditures_Software Expenditures Interlibrary Loan Expenditures

(23.a)

(23.b)

(24)
(25) (Survey Question Number)

\begin{tabular}{|c|c|c|c|c|}
\hline 318,654 & 9,566 & 391,149 & 36,206 & ALABAMA \\
\hline UA/NA & $\mathrm{UA} / \mathrm{NA}$ & $\mathrm{UA} / \mathrm{NA}$ & $\mathrm{UA} / \mathrm{NA}$ & ALBERTA \\
\hline UA/NA & $\mathrm{UA} / \mathrm{NA}$ & $2,480,111$ & 126,279 & ARIZONA \\
\hline 110,124 & 0 & 531,124 & 336,443 & ARIZONA STATE \\
\hline 176,892 & 166,500 & 711,465 & 90,860 & AUBURN \\
\hline 182,320 & $\mathrm{UA} / \mathrm{NA}$ & 284,194 & 78,423 & BOSTON \\
\hline 160,457 & $\mathrm{UA} / \mathrm{NA}$ & 181,609 & 17,751 & BOSTON COLLEGE \\
\hline 455,489 & $\mathrm{UA} / \mathrm{NA}$ & 788,030 & 49,021 & BRIGHAM YOUNG \\
\hline 341,940 & 0 & 323,749 & 142,567 & BRITISH COLUMBIA \\
\hline 0 & 8,500 & 109,090 & 169,296 & BROWN \\
\hline 383,188 & $1,098,800$ & 687,317 & 223,026 & CALIFORNIA BERKELEY \\
\hline 135,105 & 961,450 & 628,003 & 135,975 & CALIFORNIA DAVIS \\
\hline 130,549 & 892,775 & 527,696 & 78,798 & CALIFORNIA IRVINE \\
\hline 462,101 & $1,167,475$ & $2,400,378$ & 292,792 & CALIFORNIA LOS ANGELES \\
\hline 170,306 & 549,400 & 158,727 & 60,295 & CALIFORNIA RIVERSIDE \\
\hline 0 & 892,775 & $1,101,079$ & 130,711 & CALIFORNIA SAN DIEGO \\
\hline 216,000 & 618,075 & 618,759 & 164,425 & CALIFORNIA SANTA BARBARA \\
\hline 82,299 & 143,698 & 209,087 & 40,251 & CASE WESTERN RESERVE \\
\hline 383,914 & 250,000 & 516,063 & 238,702 & CHICAGO \\
\hline 150,607 & 505,148 & 904,436 & 19,344 & CINCINNATI \\
\hline 0 & 25,000 & 143,810 & 119,078 & COLORADO \\
\hline 286,767 & 260,877 & 621,548 & 284,463 & COLORADO STATE \\
\hline 527,268 & 0 & $1,358,001$ & UA/NA & COLUMBIA \\
\hline 138,092 & $1,961,515$ & 659,647 & 170,179 & CONNECTICUT \\
\hline 250,000 & 0 & $2,008,155$ & 123,486 & CORNELL \\
\hline UA/NA & $\mathrm{UA} / \mathrm{NA}$ & 226,280 & 198,151 & DARTMOUTH \\
\hline 70,582 & 0 & 333,127 & 71,834 & DELAWARE \\
\hline 314,281 & 2,969 & $1,242,884$ & 55,582 & DUKE \\
\hline 178,171 & 249,430 & 478,926 & 32,815 & EMORY \\
\hline 184,272 & 565,034 & 430,100 & 78,906 & FLORIDA \\
\hline 193,429 & 544,848 & 205,614 & 77,131 & FLORIDA STATE \\
\hline 4,600 & 93,639 & 332,007 & 105,756 & GEORGE WASHINGTON \\
\hline 183,449 & 3,726 & 388,084 & 38,279 & GEORGETOWN \\
\hline 17,284 & . & 373,334 & 694 & GEORGIA \\
\hline 175,000 & 0 & 427,254 & 289,876 & GEORGIA TECH \\
\hline 187,560 & 270,172 & 400,119 & 81,742 & GUELPH \\
\hline $2,094,192$ & $\mathrm{UA} / \mathrm{NA}$ & $1,876,259$ & $\mathrm{UA} / \mathrm{NA}$ & HARVARD \\
\hline 196,927 & 0 & 285,569 & 123,291 & HAWAII \\
\hline 503,345 & 0 & 827,493 & 22,430 & HOUSTON \\
\hline
\end{tabular}




\section{Electronic Resources Expenditures}

One-time Purchases
Ongoing Purchases
Total

ic Resource

(22) Purchases

$(21+22)$

Total Electronic Resources Library Materials Expenditures as a \% of Library Materials Budget

(Survey Question Number)

(21)

(15)

\begin{tabular}{|c|c|c|c|c|c|c|}
\hline INSTITUTION & Notes & & & & & \\
\hline HOWARD & bLM+ & 22,000 & $2,053,989$ & $2,075,989$ & $3,823,732$ & 54.29 \\
\hline ILLINOIS CHICAGO & $\mathrm{M}+$ & 105,000 & $4,541,070$ & $4,646,070$ & $9,128,496$ & 50.90 \\
\hline ILLINOIS URBANA & bGL+ & 269,350 & $5,651,861$ & $5,921,211$ & $14,065,662$ & 42.10 \\
\hline INDIANA & GLM+ & $1,230,400$ & $5,356,619$ & $6,587,019$ & $16,504,866$ & 39.91 \\
\hline IOWA & bLM+ & 356,625 & $5,937,277$ & $6,293,902$ & $13,590,872$ & 46.31 \\
\hline IOWA STATE & G+ & 73,449 & $6,283,448$ & $6,356,897$ & $9,359,327$ & 67.92 \\
\hline JOHNS HOPKINS & $\mathrm{BbG}+$ & 238,137 & $4,604,961$ & $4,843,098$ & $14,824,216$ & 32.67 \\
\hline KANSAS & $\mathrm{BbLM}+$ & $1,015,630$ & $3,915,753$ & $4,931,383$ & $9,581,850$ & 51.47 \\
\hline KENT STATE & $\mathrm{BbG}+$ & UA/NA & UA/NA & . & $4,042,951$ & \\
\hline KENTUCKY & GLM+ & 980,309 & $5,158,151$ & $6,138,460$ & $10,416,077$ & 58.93 \\
\hline LAVAL & G+ & 465,692 & $5,202,663$ & $5,668,355$ & $10,374,866$ & 54.64 \\
\hline LOUISIANA STATE & GLM+ & 787,969 & $3,484,606$ & $4,272,575$ & $7,386,879$ & 57.84 \\
\hline LOUISVILLE & $\mathrm{BbG}+$ & 73,254 & $3,138,477$ & $3,211,731$ & $9,206,025$ & 34.89 \\
\hline MCGILL & BbGLM+ & $1,289,265$ & $7,597,757$ & $8,887,022$ & $16,085,628$ & 55.25 \\
\hline MCMASTER & $\mathrm{GM}+$ & 398,745 & $4,903,903$ & $5,302,647$ & $8,261,937$ & 64.18 \\
\hline MANITOBA & G+ & $1,069,613$ & $3,319,036$ & $4,388,649$ & $8,330,789$ & 52.68 \\
\hline MARYLAND & G & 357,421 & $6,027,399$ & $6,384,820$ & $10,097,607$ & 63.23 \\
\hline MASSACHUSETTS & G+ & 328,449 & $4,061,050$ & $4,389,499$ & $6,545,019$ & 67.07 \\
\hline MIT & G+ & 654,405 & $4,660,758$ & $5,315,163$ & $8,886,555$ & 59.81 \\
\hline MIAMI & bGLM+ & 524,849 & $6,419,942$ & $6,944,791$ & $13,213,588$ & 52.56 \\
\hline MICHIGAN & GLM+ & 601,395 & $7,132,617$ & $7,734,012$ & $20,525,876$ & 37.68 \\
\hline MICHIGAN STATE & GL+ & 686,909 & $5,636,707$ & $6,323,616$ & $11,267,304$ & 56.12 \\
\hline MINNESOTA & bGLM+ & $1,219,091$ & $6,855,437$ & $8,074,528$ & $16,578,284$ & 48.71 \\
\hline MISSOURI & GL+ & 56,474 & $6,197,973$ & $6,254,447$ & $8,515,580$ & 73.45 \\
\hline MONTREAL & GLM+ & 307,828 & $6,358,934$ & $6,666,762$ & $11,294,805$ & 59.03 \\
\hline NEBRASKA & GL+ & 395,861 & $2,028,356$ & $2,424,217$ & $7,039,988$ & 34.43 \\
\hline NEW MEXICO & GLM+ & $1,725,020$ & $3,588,928$ & $5,313,948$ & $8,676,839$ & 61.24 \\
\hline NEW YORK & bGLM+ & $1,058,971$ & $8,167,089$ & $9,226,060$ & $16,498,536$ & 55.92 \\
\hline NORTH CAROLINA & GLM+ & $1,712,520$ & $3,436,539$ & $5,149,059$ & $16,322,573$ & 31.55 \\
\hline NORTH CAROLINA STATE & G+ & 502,341 & $3,328,110$ & $3,830,451$ & $9,809,078$ & 39.05 \\
\hline NORTHWESTERN & BGLM+ & 687,302 & $6,510,675$ & $7,197,977$ & $13,596,926$ & 52.94 \\
\hline NOTRE DAME & bGL+ & $1,756,322$ & $4,734,878$ & $6,491,200$ & $11,606,437$ & 55.93 \\
\hline $\mathrm{OHIO}$ & $\mathrm{BbG}+$ & 404,046 & $2,467,990$ & $2,872,036$ & $5,641,009$ & 50.91 \\
\hline OHIO STATE & bGLM+ & 288,080 & $6,302,092$ & $6,590,172$ & $13,178,838$ & 50.01 \\
\hline OKLAHOMA & GLM+ & $1,684,445$ & $2,894,609$ & $4,579,054$ & $14,081,833$ & 32.52 \\
\hline OKLAHOMA STATE & BGM+ & UA/NA & UA/NA & - & $6,841,311$ & . \\
\hline OREGON & bGL+ & 108,000 & $2,731,843$ & $2,839,843$ & $6,718,280$ & 42.27 \\
\hline PENNSYLVANIA & GLM+ & 627,031 & $6,165,221$ & $6,792,252$ & $14,759,587$ & 46.02 \\
\hline PENNSYLVANIA STATE & BGLM+ & 97,060 & $10,227,659$ & $10,324,719$ & $17,826,123$ & 57.92 \\
\hline
\end{tabular}




\section{Electronic Resources Expenditures}

Bibl. Utilities, Networks, etc. Bibl. Utilities, Networks, etc. Computer Hardware and Document Delivery/ Library Expenditures

External Expenditures

(23.b)

(24)

(23.a)

Software Expenditure
Interlibrary Loan

Expenditures
(25) (Survey Question Number)

\begin{tabular}{|c|c|c|c|c|}
\hline 113,489 & UA/NA & 91,516 & 23,500 & HOWARD \\
\hline 49,477 & UA/NA & $\mathrm{UA} / \mathrm{NA}$ & $\mathrm{UA} / \mathrm{NA}$ & ILLINOIS CHICAGO \\
\hline 439,043 & UA/NA & 576,734 & 4,301 & ILLINOIS URBANA \\
\hline 240,699 & 229,540 & 628,332 & 230,700 & INDIANA \\
\hline 176,853 & 0 & 439,535 & 274,614 & IOWA \\
\hline 97,760 & 0 & 636,034 & 171,195 & IOWA STATE \\
\hline 33,445 & 0 & 601,856 & 46,078 & JOHNS HOPKINS \\
\hline 307,363 & 0 & 428,822 & 226,855 & KANSAS \\
\hline 104,933 & 573,692 & 373,756 & 31,655 & KENT STATE \\
\hline 223,550 & 0 & 521,443 & 201,929 & KENTUCKY \\
\hline . & . & . & 54,625 & LAVAL \\
\hline 330,080 & 139,280 & 67,776 & 71,668 & LOUISIANA STATE \\
\hline & 233,589 & 308,469 & 30,140 & LOUISVILLE \\
\hline 359,754 & . & 519,734 & 34,282 & MCGILL \\
\hline 95,095 & 0 & 111,791 & 94,739 & MCMASTER \\
\hline 86,867 & 0 & 281,882 & 165,995 & MANITOBA \\
\hline 605,392 & 164,219 & 255,941 & 307,988 & MARYLAND \\
\hline 248,033 & 75,000 & 232,637 & 124,399 & MASSACHUSETTS \\
\hline 100,163 & . & 681,696 & 92,627 & MIT \\
\hline 222,630 & 0 & $1,405,206$ & 153,651 & MIAMI \\
\hline 395,495 & 3,500 & $2,304,437$ & 223,325 & MICHIGAN \\
\hline 308,019 & UA/NA & 628,771 & 252,626 & MICHIGAN STATE \\
\hline 234,652 & 240,800 & $1,881,259$ & 269,076 & MINNESOTA \\
\hline 129,135 & 0 & 56,055 & 37,950 & MISSOURI \\
\hline 28,851 & 665,134 & 607,667 & 194,248 & MONTREAL \\
\hline 139,289 & 125,887 & 403,993 & 64,449 & NEBRASKA \\
\hline 558,946 & 1,270 & 431,718 & 49,892 & NEW MEXICO \\
\hline $\mathrm{UA} / \mathrm{NA}$ & $\mathrm{UA} / \mathrm{NA}$ & $1,765,378$ & 264,193 & NEW YORK \\
\hline 393,290 & $\mathrm{UA} / \mathrm{NA}$ & 999,092 & 160,165 & NORTH CAROLINA \\
\hline 176,002 & 0 & 792,904 & 143,756 & NORTH CAROLINA STATE \\
\hline 217,825 & UA/NA & 740,079 & 143,905 & NORTHWESTERN \\
\hline 130,862 & 182,643 & 645,589 & 77,587 & NOTRE DAME \\
\hline 152,065 & 499,209 & 278,180 & 49,007 & $\mathrm{OHIO}$ \\
\hline 154,599 & $1,185,462$ & 603,671 & 128,485 & OHIO STATE \\
\hline 282,439 & 42,891 & 674,296 & 98,729 & OKLAHOMA \\
\hline UA/NA & UA/NA & 707,540 & 96,170 & OKLAHOMA STATE \\
\hline 254,616 & 39,547 & 716,823 & 127,855 & OREGON \\
\hline $\mathrm{UA} / \mathrm{NA}$ & 718,144 & 992,209 & 477,681 & PENNSYLVANIA \\
\hline 335,103 & 20,979 & $1,825,664$ & 209,813 & PENNSYLVANIA STATE \\
\hline
\end{tabular}




\section{Electronic Resources Expenditures}

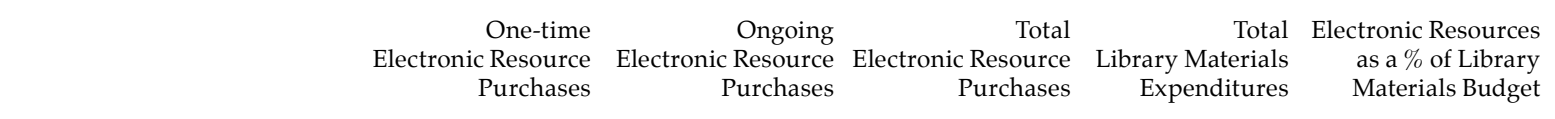

(Survey Question Number)

(21)

(22)

$(21+22)$

(15)

\section{INSTITUTION}

PITTSBURGH

PRINCETON

PURDUE

QUEENS

RICE

ROCHESTER

RUTGERS

SASKATCHEWAN

SOUTH CAROLINA

SOUTHERN CALIFORNIA

SOUTHERN ILLINOIS

SUNY-ALBANY

SUNY-BUFFALO

SUNY-STONY BROOK

SYRACUSE

TEMPLE

TENNESSEE

TEXAS

TEXAS A\&M

TEXAS TECH

TORONTO

TULANE

UTAH

VANDERBILT

VIRGINIA

VIRGINIA TECH

WASHINGTON

WASHINGTON STATE

WASHINGTON U.-ST. LOUIS

WATERLOO

WAYNE STATE

WESTERN ONTARIO

WISCONSIN

YALE

YORK

BOSTON PUBLIC

CISTI

CENTER FOR RESEARCH LIBRARIES +

LIBRARY OF CONGRESS
Notes

BGLM+ 494,775

bG+ $\quad 587,551$

G+

47,966

$\mathrm{LM}+$

UA/NA

G+

$1,122,258$

bM+

140,508

BGL+

311,558

GLM+

33,215

LM+

310,756

BGLM+

$1,266,271$

LM+

76,295

G+

GLM+

123,778

90,283

$\mathrm{M}+$

190,481

GL+

233,109

BbGLM+

$1,447,647$

BbGLM+

551,622

GL+

$1,969,344$

$\mathrm{BbG}+$

1,727,698

BLM+

618,470

B+

BLM+

$1,717,881$

LM

LM+

GLM+

416,358

G

GLM+

UA/NA

427,557

425,193

BG+

24,644

438,415

bGLM+

47,257

G+

489,836

122,520

$+$

$+$

441,828

GL+ $\quad 55,569$

GLM+

LM+

473,186

BGL+

746,595

$1,651,447$

G

$+\quad 301,657$

5,512

bG+

$1,195,983$

$9,082,800$

$9,082,800$
$7,806,016$

$6,345,104$

$5,671,832$

$3,635,342$

$3,391,125$

$4,696,278$

$5,508,447$

$4,602,065$

$4,673,283$

$3,852,031$

$1,836,463$

$5,387,476$

$3,753,464$

$3,556,347$

$5,154,310$

$5,091,930$

$6,483,628$

$7,383,376$

$4,309,998$

7,565,335

4,043,292

2,077,201

$7,189,423$

5,336,630

4,417,080

$7,075,116$

$4,097,898$

$6,289,732$

$3,599,741$

$7,529,660$

$6,922,605$

4,891,636

$6,608,437$

4,463,680

$1,067,944$

1,960,754

UA/NA

2,060,000

$9,577,575$

$8,393,567$

6,393,070

$5,671,832$

$4,757,600$

$3,531,633$

$5,007,836$

$5,541,661$

$4,912,821$

$5,939,554$

$3,928,326$

$1,960,241$

$5,477,759$

$3,943,945$

$3,789,456$

6,601,957

$5,643,552$

$8,452,972$

9,111,074

$4,928,468$

$9,283,217$

$4,459,650$

2,077,201

$7,616,980$

$5,761,823$

$4,441,724$

$7,513,531$

4,145,155

$6,779,568$

$3,722,260$

$7,971,488$

$6,978,173$

$5,364,822$

$7,355,032$

$6,115,127$

$1,114,529$

2,262,412

5,512

$3,255,983$ 


\section{Electronic Resources Expenditures}

Bibl. Utilities, Networks, etc. Bibl. Utilities, Networks, etc. Computer Hardware and Document Delivery/ Library Expenditures

External Expenditures

(23.b)

(24)

(23.a)

Software Expenditures
Interlibrary Loan

Expenditures
(25) (Survey Question Number)

\begin{tabular}{|c|c|c|c|c|}
\hline UA/NA & 315,912 & 777,806 & 153,555 & PITTSBURGH \\
\hline 393,649 & 0 & $1,962,175$ & 147,973 & PRINCETON \\
\hline 345,746 & 253,834 & 757,772 & 182,657 & PURDUE \\
\hline 157,709 & 100,363 & 366,216 & 30,520 & QUEENS \\
\hline 139,967 & 0 & 383,975 & 34,991 & RICE \\
\hline 180,103 & 0 & $1,203,591$ & 71,143 & ROCHESTER \\
\hline 235,673 & $\mathrm{UA} / \mathrm{NA}$ & 377,612 & 82,387 & RUTGERS \\
\hline 101,751 & UA/NA & 340,202 & 92,576 & SASKATCHEWAN \\
\hline 187,428 & 0 & 478,049 & 75,215 & SOUTH CAROLINA \\
\hline 340,548 & 0 & $1,991,889$ & 107,677 & SOUTHERN CALIFORNIA \\
\hline 204,027 & 92,717 & 245,180 & 132,195 & SOUTHERN ILLINOIS \\
\hline 49,916 & 70,516 & 138,046 & 115,038 & SUNY-ALBANY \\
\hline 337,568 & UA/NA & 303,223 & $\mathrm{UA} / \mathrm{NA}$ & SUNY-BUFFALO \\
\hline $\mathrm{UA} / \mathrm{NA}$ & $\mathrm{UA} / \mathrm{NA}$ & 507,437 & 29,638 & SUNY-STONY BROOK \\
\hline 18,722 & 0 & 369,526 & 106,040 & SYRACUSE \\
\hline 147,649 & 9,000 & 948,171 & 142,957 & TEMPLE \\
\hline 32,477 & 13,528 & 361,296 & 166,475 & TENNESSEE \\
\hline 161,770 & 0 & $1,332,899$ & 285,135 & TEXAS \\
\hline 0 & 469,296 & $1,405,383$ & 336,397 & TEXAS A\&M \\
\hline 232,582 & 54,750 & $1,784,010$ & 55,598 & TEXAS TECH \\
\hline 720,046 & $\mathrm{UA} / \mathrm{NA}$ & $1,973,614$ & 97,693 & TORONTO \\
\hline 157,246 & 88,248 & 179,658 & 12,765 & TULANE \\
\hline 231,932 & $1,874,004$ & 996,062 & 87,030 & UTAH \\
\hline 244,623 & UA/NA & 600,534 & 126,389 & VANDERBILT \\
\hline 185,530 & 729,533 & $1,697,955$ & 190,990 & VIRGINIA \\
\hline 103,726 & 0 & 184,178 & 268,879 & VIRGINIA TECH \\
\hline 265,228 & 139,173 & 380,990 & 240,925 & WASHINGTON \\
\hline 105,396 & 76,857 & 217,843 & 30,039 & WASHINGTON STATE \\
\hline 255,098 & 361 & 960,484 & 27,985 & WASHINGTON U.-ST. LOUIS \\
\hline 16,277 & 0 & 460,740 & 56,577 & WATERLOO \\
\hline 143,224 & 0 & $1,045,972$ & 133,757 & WAYNE STATE \\
\hline 185,814 & 0 & 341,867 & 12,061 & WESTERN ONTARIO \\
\hline 363,992 & 408,990 & $1,831,951$ & 424,355 & WISCONSIN \\
\hline 254,864 & 0 & 939,460 & 230,647 & YALE \\
\hline 76,625 & UA/NA & 272,787 & 24,420 & YORK \\
\hline $11,288,398$ & $23,401,050$ & UA/NA & UA/NA & BOSTON PUBLIC \\
\hline $\mathrm{UA} / \mathrm{NA}$ & UA/NA & $2,254,137$ & $\mathrm{UA} / \mathrm{NA}$ & CISTI \\
\hline 203,957 & UA/NA & 163,787 & 193,249 & CENTER FOR RESEARCH LIBRARIES \\
\hline 669,623 & 0 & $17,500,000$ & 11,000 & LIBRARY OF CONGRESS \\
\hline
\end{tabular}




\section{Electronic Resources Expenditures}

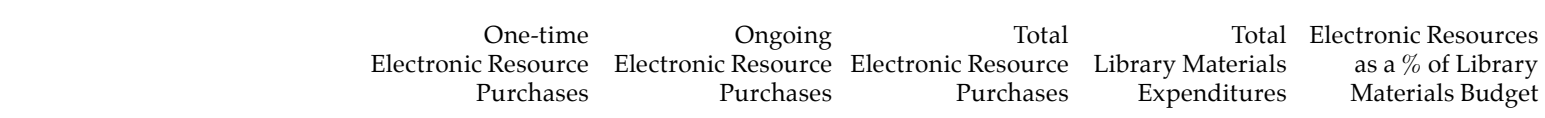

(Survey Question Number)

(21)

(22)

$(21+22)$

(15)

INSTITUTION

Notes

\begin{tabular}{lcrrrrr}
\hline \multicolumn{2}{l}{ NATIONAL AGRICULTURAL LIBRARY bG+ } & 67,833 & $2,473,923$ & $2,541,756$ & $3,786,097$ & 67.13 \\
LIBRARY \& ARCHIVES CANADA & $\mathrm{G}+$ & UA/NA & 38,460 & 38,460 & $1,805,837$ & 2.13 \\
NATIONAL LIBRARY OF MEDICINE & $\mathrm{G}+$ & 2,513 & 152,963 & 155,476 & $9,053,656$ \\
NEW YORK PUBLIC & + & $\mathrm{UA} / \mathrm{NA}$ & $2,893,110$ & $2,893,110$ & $15,224,911$ \\
NEW YORK STATE & $\mathrm{G}+$ & 0 & 449,200 & 449,200 & $4,270,000$ \\
SMITHSONIAN & $\mathrm{G}+$ & 139,505 & 165,357 & 304,862 & $1,560,755$ & 19.00
\end{tabular}

+ - See Footnotes

L - Includes Law library

M- Includes Medical library
G - Government documents not included in serials count B - Includes branch campuses

$\mathrm{b}-$ Basis of volume count is bibliographic
U/A - Unavailable

N/A - Not Applicable

. - no data supplied

\section{SumMary Data}

\begin{tabular}{|c|c|c|c|c|}
\hline ime & jing & Total & Total & ic $\mathrm{R}$ \\
\hline $\begin{array}{r}\text { Electronic Resource } \\
\text { Purchases }\end{array}$ & $\begin{array}{r}\text { Electronic Resource } \\
\text { Purchases }\end{array}$ & $\begin{array}{l}\text { Electronic Resource } \\
\text { Purchases }\end{array}$ & $\begin{array}{l}\text { Library Materials } \\
\text { Expenditures }\end{array}$ & $\begin{array}{l}\text { as a \% of Library } \\
\text { Materials Budget }\end{array}$ \\
\hline
\end{tabular}

(Survey Question Number)

(21)

(22)

$(21+22)$

(15)

UNIVERSITY LIBRARIES

$\begin{array}{lrrrrr}\text { Mean } & 676,574 & 5,042,162 & 5,655,026 & 11,421,020 & 10,388,129 \\ \text { Median } & 410,202 & 4,899,366 & 5,410,421 & 34,291,329 \\ \text { High } & 8,300,095 & 10,227,659 & 14,521,604 & 53.06 \\ \text { Low } & 0 & 1,836,463 & 1,960,241 & 3,823,732 \\ \text { Totals } & 73,070,024 & 554,637,844 & 627,707,869 & 1,290,575,224 \\ \text { Number of Libraries Reporting } & 108 & 110 & 111 & 22.06 \\ & & & 113\end{array}$

NoNUNIVERSITY LibRARIES

\author{
Median \\ Totals \\ Number of Libraries Reporting
}

$$
57,209
$$

$1,759,588$

8
$1,067,944$

$11,261,711$

9

$565,899,555$
781,865

13,021,299

10

$74,829,612$

$640,729,168$
5,182,164

17.87

$72,894,534$

10 as a \% of Library
Materials Budget

\section{GRAND TOTALS}

$1,363,469,758$ 


\section{Electronic Resources Expenditures}

Bibl. Utilities, Networks, etc. Library Expenditures
Bibl. Utilities, Networks, etc. External Expenditures
Computer Hardware and Software Expenditures
Document Delivery/ Interlibrary Loan Expenditures (23.a)

(23.b)
(24)
(25) (Survey Question Number)

INSTITUTION

\begin{tabular}{rrrrr}
\hline 0 & 3 & 536,190 & 599,273 & NATIONAL AGRICULTURAL LIBRARY \\
UA/NA & UA/NA & $14,871,427$ & 11,194 & LIBRARY \& ARCHIVES CANADA \\
UA/NA & UA/NA &. & NATIONAL LIBRARY OF MEDICINE \\
278,210 & UA/NA & 891,922 & UA/NA & NEW YORK PUBLIC \\
UA/NA & 91,249 & 209,099 & UA/NA & NEW YORK STATE \\
51,961 & 0 & 55,922 & 2,330 & SMITHSONIAN
\end{tabular}

\section{Summary Data} Bibl. Utilities, Networks, etc.
Library Expenditures
Bibl. Utilities, Networks, etc. External Expenditures
Computer Hardware and Software Expenditures (23.a)

(23.b)

(24)

Document Delivery/ Interlibrary Loan Expenditures

\begin{tabular}{|c|c|c|c|c|}
\hline & & & & UNIVERSITY LIBRARIES \\
\hline 229,785 & 242,290 & 722,794 & 129,883 & Mean \\
\hline 184,272 & 54,750 & 529,410 & 111,358 & Median \\
\hline $2,094,192$ & $1,961,515$ & $2,480,111$ & 477,681 & High \\
\hline 0 & 0 & 56,055 & 694 & Low \\
\hline $23,667,883$ & $21,079,241$ & $79,507,314$ & $14,027,391$ & Totals \\
\hline \multirow[t]{2}{*}{103} & 87 & 110 & 108 & Number of Libraries Reporting \\
\hline & & & & NONUNIVERSITY LIBRARIES \\
\hline $2,082,025$ & $4,698,460$ & $4,560,310$ & 163,409 & Median \\
\hline 241,084 & 3 & 714,056 & 11,194 & Totals \\
\hline 6 & 5 & 8 & 5 & Number of Libraries Reporting \\
\hline $36,160,032$ & $44,571,543$ & $115,989,798$ & $14,844,437$ & GRAND TOTALS \\
\hline
\end{tabular}




\section{Personnel and Public Services}

Professional Staff Support Staff Student Assistants Total Staff Staffed Library $\begin{array}{llll}(\text { FTE }) & \text { (FTE) }\end{array}$

(26.c)

(26)

(27)

(28)

INSTITUTION

\begin{tabular}{|c|c|c|c|c|c|c|c|}
\hline ALABAMA & bGLM+ & 70 & 83 & 49 & 202 & 22 & 132 \\
\hline ALBERTA & G+ & 85 & 213 & 36 & 334 & 19 & 103 \\
\hline ARIZONA & bGLM+ & 94 & 146 & 74 & 314 & 10 & 142 \\
\hline ARIZONA STATE & BGL+ & 93 & 195 & 44 & 332 & 20 & 149 \\
\hline AUBURN & bG+ & 46 & 40 & 31 & 117 & 11 & 146 \\
\hline BOSTON & LM+ & 102 & 97 & 86 & 285 & 32 & 108 \\
\hline BOSTON COLLEGE & $\mathrm{L}+$ & 79 & 73 & 50 & 202 & 15 & 110 \\
\hline BRIGHAM YOUNG & $\mathrm{L}+$ & 120 & 59 & 204 & 383 & 19 & 105 \\
\hline BRITISH COLUMBIA & G+ & 114 & 186 & 38 & 338 & 31 & 101 \\
\hline BROWN & bG+ & 73 & 101 & 34 & 208 & 8 & 112 \\
\hline CALIFORNIA BERKELEY & GL+ & 244 & 198 & 140 & 582 & 49 & 77 \\
\hline CALIFORNIA DAVIS & GLM+ & 59 & 144 & 46 & 249 & 16 & 95 \\
\hline CALIFORNIA IRVINE & bGM+ & 73 & 127 & 48 & 248 & 14 & 128 \\
\hline CALIFORNIA LOS ANGELES & $\mathrm{LM}+$ & 169 & 281 & 147 & 597 & 30 & 97 \\
\hline CALIFORNIA RIVERSIDE & $\mathrm{G}^{+}$ & 49 & 79 & 31 & 159 & 14 & 91 \\
\hline CALIFORNIA SAN DIEGO & bGM+ & 104 & 193 & 70 & 367 & 18 & 114 \\
\hline CALIFORNIA SANTA BARBARA & G+ & 47 & 135 & 47 & 229 & 10 & 103 \\
\hline CASE WESTERN RESERVE & GLM+ & 58 & 45 & 28 & 131 & 18 & 104 \\
\hline CHICAGO & bGLM+ & 68 & 192 & 63 & 323 & 29 & 146 \\
\hline CINCINNATI & bGLM+ & 76 & 58 & 48 & 182 & 22 & 95 \\
\hline COLORADO & bGL+ & 58 & 109 & 49 & 216 & 13 & 104 \\
\hline COLORADO STATE & $\mathrm{G}+$ & 51 & 54 & 25 & 130 & 7 & 108 \\
\hline COLUMBIA & GLM+ & 328 & 246 & 127 & 701 & 47 & 108 \\
\hline CONNECTICUT & BGLM+ & 86 & 72 & 49 & 207 & 20 & 114 \\
\hline CORNELL & BGL+ & 120 & 308 & 121 & 549 & 53 & 146 \\
\hline DARTMOUTH & GM+ & 60 & 117 & 28 & 205 & 19 & 123 \\
\hline DELAWARE & G+ & 58 & 105 & 35 & 198 & 16 & 100 \\
\hline DUKE & bLM+ & 185 & 143 & 35 & 363 & 19 & 161 \\
\hline EMORY & BGLM+ & 94 & 153 & 49 & 296 & 29 & 118 \\
\hline FLORIDA & BbGLM+ & 102 & 190 & 89 & 381 & 19 & 105 \\
\hline FLORIDA STATE & BbLM+ & 68 & 110 & 70 & 248 & 24 & 142 \\
\hline GEORGE WASHINGTON & GLM+ & 84 & 124 & 78 & 286 & 26 & 168 \\
\hline GEORGETOWN & bGLM+ & 95 & 116 & 47 & 258 & 20 & 107 \\
\hline GEORGIA & GL+ & 81 & 184 & 39 & 304 & 22 & 137 \\
\hline GEORGIA TECH & $\mathrm{G}+$ & 45 & 70 & 8 & 123 & 8 & 135 \\
\hline GUELPH & BG+ & 54 & 69 & 21 & 144 & 12 & 116 \\
\hline HARVARD & GLM+ & 555 & 541 & 133 & 1,229 & UA/NA & UA/NA \\
\hline HAWAII & GLM+ & 92 & 77 & 67 & 236 & 16 & 135 \\
\hline HOUSTON & $\mathrm{bL}+$ & 62 & 91 & 46 & 199 & 8 & 108 \\
\hline
\end{tabular}




\section{Personnel and Public Services}

Library Participants Reference Initial Total Total Items Total Items Presentations in Group Transactions* Circulation Circulation Loaned Borrowed to Groups* Presentations* Transactions Transactions (ILL) (ILL)
(29)
(30)
(31)
(32)
(33)
(34)
(35) (Survey Question Number)

INSTITUTION

\begin{tabular}{|c|c|c|c|c|c|c|c|}
\hline 584 & 11,689 & 58,763 & 193,211 & 243,421 & 12,114 & 9,799 & ALABAMA \\
\hline 863 & 21,708 & 87,630 & 485,108 & 828,100 & 53,211 & 30,463 & ALBERTA \\
\hline 2,318 & 33,590 & 42,644 & 291,500 & 459,935 & 55,343 & 36,714 & ARIZONA \\
\hline 1,295 & 27,574 & 106,751 & 293,252 & 841,179 & 50,966 & 38,561 & ARIZONA STATE \\
\hline 750 & 15,000 & 159,796 & 157,928 & 194,083 & 12,091 & 8,651 & AUBURN \\
\hline 1,284 & 21,066 & 47,476 & 190,498 & 252,938 & 20,839 & 21,266 & BOSTON \\
\hline 665 & 14,457 & 30,481 & 182,703 & 293,754 & 28,636 & 22,253 & BOSTON COLLEGE \\
\hline 2,523 & 48,648 & 159,342 & 484,093 & 641,592 & 47,231 & 25,637 & BRIGHAM YOUNG \\
\hline 1,496 & 29,253 & 108,538 & 640,701 & $2,608,952$ & 22,884 & 22,542 & BRITISH COLUMBIA \\
\hline 349 & 5,374 & 25,512 & 197,939 & 301,192 & 41,359 & 40,355 & BROWN \\
\hline 2,005 & 31,846 & 114,466 & 748,142 & 911,834 & 42,769 & 21,922 & CALIFORNIA BERKELEY \\
\hline 700 & 13,732 & 76,234 & 249,669 & 458,853 & 32,098 & 26,504 & CALIFORNIA DAVIS \\
\hline 695 & 18,652 & 72,852 & 156,924 & 353,165 & 27,869 & 31,034 & CALIFORNIA IRVINE \\
\hline 1,008 & 21,031 & 161,533 & 684,563 & $2,223,990$ & 49,510 & 34,596 & CALIFORNIA LOS ANGELES \\
\hline 892 & 13,355 & 26,157 & 133,528 & 230,098 & 18,928 & 14,186 & CALIFORNIA RIVERSIDE \\
\hline 1,821 & 38,892 & 59,563 & 446,615 & 680,282 & 37,556 & 28,588 & CALIFORNIA SAN DIEGO \\
\hline 611 & 11,088 & 65,468 & 243,574 & 399,306 & 24,050 & 37,282 & CALIFORNIA SANTA BARBARA \\
\hline 539 & 6,720 & 18,901 & 116,599 & 193,424 & 52,249 & 35,150 & CASE WESTERN RESERVE \\
\hline 477 & 6,664 & 25,964 & 326,159 & 565,318 & 50,664 & 22,312 & CHICAGO \\
\hline 1,133 & 21,341 & 77,915 & 242,413 & 358,654 & 62,455 & 42,020 & CINCINNATI \\
\hline 726 & 13,259 & 71,449 & 333,350 & 564,016 & 59,882 & 30,159 & COLORADO \\
\hline 474 & 10,708 & 73,148 & 192,654 & 336,483 & 70,733 & 66,476 & COLORADO STATE \\
\hline 1,363 & 25,344 & 201,408 & 429,272 & 922,081 & 46,893 & 55,114 & COLUMBIA \\
\hline 2,249 & 19,167 & 34,177 & 159,022 & 173,899 & 41,259 & 42,188 & CONNECTICUT \\
\hline 1,564 & 22,012 & 74,830 & 433,034 & 865,668 & 56,865 & 33,528 & CORNELL \\
\hline UA/NA & UA/NA & UA/NA & 279,711 & 362,250 & 35,464 & 30,481 & DARTMOUTH \\
\hline 424 & 7,513 & 104,932 & $\mathrm{UA} / \mathrm{NA}$ & 372,683 & 17,207 & 23,688 & DELAWARE \\
\hline 1,186 & 12,862 & 137,488 & 433,208 & 642,487 & 33,879 & 30,179 & DUKE \\
\hline 1,220 & 15,723 & 57,709 & 207,102 & 534,329 & 36,602 & 21,068 & EMORY \\
\hline 739 & 20,866 & 102,000 & 419,151 & 700,074 & 25,472 & 28,823 & FLORIDA \\
\hline 913 & 15,976 & 87,692 & 272,599 & 386,637 & 18,785 & 14,990 & FLORIDA STATE \\
\hline 1,317 & 20,827 & 36,464 & 174,114 & 294,072 & 32,010 & 53,439 & GEORGE WASHINGTON \\
\hline 1,211 & 13,586 & 76,730 & 273,968 & 423,602 & 37,306 & 24,366 & GEORGETOWN \\
\hline 1,266 & 15,763 & 61,731 & 286,878 & 435,650 & 42,723 & 6,862 & GEORGIA \\
\hline 289 & 6,302 & 8,178 & 105,790 & 105,790 & 10,598 & 12,062 & GEORGIA TECH \\
\hline 329 & 10,939 & 17,140 & 161,093 & 428,200 & 45,774 & 54,470 & GUELPH \\
\hline UA/NA & UA/NA & UA/NA & UA/NA & $1,986,380$ & 44,808 & 12,877 & HARVARD \\
\hline 345 & 6,145 & 45,449 & 248,979 & 449,698 & 6,246 & 31,778 & HAWAII \\
\hline 355 & 7,282 & 29,210 & 118,197 & 198,410 & 26,008 & 18,395 & HOUSTON \\
\hline
\end{tabular}




\section{Personnel and Public Services}

Professional Staff Support Staff Student Assistants Total Staff Staffed Library $\begin{array}{llll}\text { (FTE) } & \text { (FTE) } & \text { (FTE) } & \text { (FTE) }\end{array}$

Service Service

Points Hours

(Survey Question Number)

(26.a)

(26.b)

(26.c)

(26)

(27)

(28)

\begin{tabular}{|c|c|c|c|c|c|c|c|}
\hline INSTITUTION & Notes & & & & & & \\
\hline HOWARD & bLM+ & 50 & 67 & 59 & 176 & 24 & 112 \\
\hline ILLINOIS CHICAGO & $\mathrm{M}+$ & 71 & 104 & 28 & 203 & 27 & 102 \\
\hline ILLINOIS URBANA & bGL+ & 199 & 194 & 119 & 512 & 42 & 144 \\
\hline INDIANA & GLM+ & 173 & 181 & 116 & 470 & 48 & 168 \\
\hline IOWA & bLM+ & 98 & 125 & 58 & 281 & 17 & 113 \\
\hline IOWA STATE & G+ & 52 & 88 & 34 & 174 & 12 & 105 \\
\hline JOHNS HOPKINS & $\mathrm{BbG}+$ & 136 & 139 & 42 & 317 & 31 & 120 \\
\hline KANSAS & BbLM+ & 103 & 108 & 71 & 282 & 10 & 140 \\
\hline KENT STATE & $\mathrm{BbG}+$ & 56 & 36 & 192 & 284 & 13 & 99 \\
\hline KENTUCKY & GLM+ & 91 & 136 & 59 & 286 & 22 & 135 \\
\hline LAVAL & G+ & 60 & 153 & 1 & 214 & 18 & 88 \\
\hline LOUISIANA STATE & GLM+ & 52 & 101 & 39 & 192 & 15 & 113 \\
\hline LOUISVILLE & $\mathrm{BbG}+$ & 53 & 95 & 40 & 188 & 14 & 168 \\
\hline MCGILL & BbGLM+ & 83 & 172 & 39 & 294 & 31 & 86 \\
\hline MCMASTER & $\mathrm{GM}+$ & 45 & 95 & 23 & 163 & 13 & 120 \\
\hline MANITOBA & $\mathrm{G}^{+}$ & 64 & 145 & 24 & 233 & 32 & 100 \\
\hline MARYLAND & G & 119 & 95 & 44 & 258 & 8 & 140 \\
\hline MASSACHUSETTS & G+ & 56 & 73 & 61 & 190 & 14 & 142 \\
\hline MIT & G+ & 103 & 94 & 17 & 214 & 11 & 104 \\
\hline MIAMI & bGLM+ & 82 & 133 & 62 & 277 & 21 & 122 \\
\hline MICHIGAN & GLM+ & 169 & 305 & 96 & 570 & 57 & 168 \\
\hline MICHIGAN STATE & GL+ & 82 & 122 & 84 & 288 & 21 & 148 \\
\hline MINNESOTA & bGLM+ & 111 & 200 & 83 & 394 & 46 & 100 \\
\hline MISSOURI & GL+ & 58 & 110 & 30 & 198 & 26 & 114 \\
\hline MONTREAL & GLM+ & 110 & 258 & UA/NA & 368 & 66 & 97 \\
\hline NEBRASKA & GL+ & 54 & 106 & 44 & 204 & 25 & 96 \\
\hline NEW MEXICO & GLM+ & 70 & 143 & 54 & 267 & 19 & 95 \\
\hline NEW YORK & bGLM+ & 139 & 227 & 92 & 458 & 30 & 119 \\
\hline NORTH CAROLINA & GLM+ & 148 & 209 & 95 & 452 & 25 & 146 \\
\hline NORTH CAROLINA STATE & G+ & 124 & 105 & 39 & 268 & 14 & 146 \\
\hline NORTHWESTERN & BGLM+ & 120 & 140 & 84 & 344 & 30 & 126 \\
\hline NOTRE DAME & bGL+ & 70 & 154 & 36 & 260 & 19 & 126 \\
\hline $\mathrm{OHIO}$ & $\mathrm{BbG}+$ & 46 & 70 & 37 & 153 & 16 & 146 \\
\hline OHIO STATE & bGLM+ & 143 & 156 & 171 & 470 & 28 & 168 \\
\hline OKLAHOMA & GLM+ & 60 & 97 & 75 & 232 & 22 & 117 \\
\hline OKLAHOMA STATE & BGM+ & 66 & 76 & 71 & 213 & 11 & 119 \\
\hline OREGON & bGL+ & 70 & 87 & 60 & 217 & 22 & 106 \\
\hline PENNSYLVANIA & GLM+ & 137 & 179 & 86 & 402 & 38 & 116 \\
\hline PENNSYLVANIA STATE & BGLM+ & 162 & 375 & 71 & 608 & 63 & 168 \\
\hline
\end{tabular}




\section{Personnel and Public Services}

Library Participants Reference Initial Total Total Items Total Items Presentations in Group Transactions* Circulation Circulation Loaned Borrowed to Groups* Presentations* Transactions Transactions (ILL) (ILL)
(29)
(30)
(31)
(32)
(33)
(34)
(35) (Survey Question Number)

INSTITUTION

\begin{tabular}{|c|c|c|c|c|c|c|c|}
\hline 294 & 6,296 & 149,962 & 158,712 & 173,121 & 8,481 & 5,560 & HOWARD \\
\hline 753 & 13,994 & 69,160 & 162,886 & 490,423 & 61,975 & 56,349 & ILLINOIS CHICAGO \\
\hline 1,197 & 21,975 & 179,742 & 489,573 & 981,041 & 93,584 & 78,457 & ILLINOIS URBANA \\
\hline 1,351 & 32,603 & 147,412 & 669,872 & 770,510 & 50,325 & 44,360 & INDIANA \\
\hline 1,168 & 21,342 & 83,755 & 328,790 & 422,053 & 62,404 & 28,607 & IOWA \\
\hline 390 & 10,112 & 25,244 & 199,569 & 243,108 & 29,651 & 17,027 & IOWA STATE \\
\hline 754 & 9,169 & 61,210 & 272,011 & 459,053 & 37,076 & 39,481 & JOHNS HOPKINS \\
\hline 1,225 & 25,981 & 107,116 & 355,299 & 506,453 & 50,502 & 39,672 & KANSAS \\
\hline 523 & 9,148 & 24,683 & 244,835 & 381,486 & 14,897 & 9,333 & KENT STATE \\
\hline 744 & 7,898 & 34,718 & 198,091 & 262,913 & 32,008 & 24,349 & KENTUCKY \\
\hline 739 & 12,468 & 142,931 & 518,540 & 797,309 & 17,842 & 9,720 & LAVAL \\
\hline 801 & 6,433 & 37,028 & 104,482 & 206,871 & 7,381 & 16,916 & LOUISIANA STATE \\
\hline 705 & 12,799 & 56,692 & 294,284 & 337,355 & 23,048 & 20,210 & LOUISVILLE \\
\hline 1,717 & 5,628 & 127,201 & 525,452 & 881,918 & 21,768 & 16,593 & MCGILL \\
\hline 709 & 16,473 & 40,066 & 261,682 & 331,183 & 15,678 & 12,726 & MCMASTER \\
\hline 1,157 & 12,837 & 87,999 & 262,105 & 449,558 & 20,917 & 25,400 & MANITOBA \\
\hline 1,062 & 23,253 & 259,320 & 297,813 & 513,464 & 22,215 & 40,713 & MARYLAND \\
\hline 557 & 11,363 & 35,918 & 182,536 & 301,617 & 44,946 & 48,539 & MASSACHUSETTS \\
\hline 348 & 7,593 & 32,497 & 188,434 & 347,504 & 19,287 & 17,464 & MIT \\
\hline 1,940 & 22,579 & 75,035 & 169,923 & 237,737 & 41,524 & 13,738 & MIAMI \\
\hline 1,269 & 22,200 & 150,010 & 568,180 & $1,432,386$ & 59,885 & 42,978 & MICHIGAN \\
\hline 983 & 38,957 & 49,351 & 296,148 & 667,563 & 62,153 & 30,895 & MICHIGAN STATE \\
\hline 1,378 & 24,107 & 97,340 & 369,736 & 733,140 & 165,913 & 41,800 & MINNESOTA \\
\hline 787 & 11,798 & 38,051 & 199,411 & 265,427 & 55,210 & 41,231 & MISSOURI \\
\hline 1,059 & 17,142 & 74,747 & 429,291 & 855,891 & 22,041 & 19,917 & MONTREAL \\
\hline 267 & 7,299 & 38,283 & 204,456 & 301,743 & 33,959 & 28,757 & NEBRASKA \\
\hline 1,451 & 20,968 & 35,270 & 187,849 & 273,857 & 21,224 & 26,959 & NEW MEXICO \\
\hline 1,057 & 17,120 & 107,795 & 506,906 & 900,456 & 33,089 & 36,634 & NEW YORK \\
\hline 1,367 & 25,785 & 126,165 & 613,417 & 974,541 & 59,026 & 14,975 & NORTH CAROLINA \\
\hline 446 & 11,977 & 32,325 & 228,167 & 358,448 & 14,979 & 22,509 & NORTH CAROLINA STATE \\
\hline 811 & 9,861 & 71,370 & 203,364 & 316,230 & 36,383 & 28,342 & NORTHWESTERN \\
\hline 422 & 6,968 & 20,749 & 206,640 & 362,709 & 29,584 & 24,050 & NOTRE DAME \\
\hline 813 & 13,127 & 62,007 & 287,355 & 486,644 & 41,847 & 49,452 & $\mathrm{OHIO}$ \\
\hline 1,235 & 21,254 & 73,818 & 407,874 & $1,530,016$ & 170,711 & 96,771 & OHIO STATE \\
\hline 1,003 & 10,581 & 45,373 & 170,743 & 185,367 & 35,148 & 32,030 & OKLAHOMA \\
\hline 1,461 & 32,621 & 110,399 & 333,133 & 368,430 & 37,091 & 21,358 & OKLAHOMA STATE \\
\hline 516 & 9,948 & 57,117 & 209,618 & 270,044 & 80,560 & 61,746 & OREGON \\
\hline 1,222 & 22,485 & UA/NA & 416,739 & 538,760 & 74,659 & 83,836 & PENNSYLVANIA \\
\hline 2,911 & 58,734 & 181,023 & 569,429 & 959,584 & 66,704 & 53,334 & PENNSYLVANIA STATE \\
\hline
\end{tabular}




\section{Personnel and Public Services}

Professional Staff Support Staff Student Assistants Total Staff Staffed Library $\begin{array}{llll}\text { (FTE) } \quad \text { (FTE) } & \text { (FTE) }\end{array}$

Service Service

Points Hours

(Survey Question Number)

(26.a)

(26.b)

(26.c)

(26)

(27)

(28)

\begin{tabular}{|c|c|c|c|c|c|c|c|}
\hline INSTITUTION & Notes & & & & & & \\
\hline PITTSBURGH & BGLM+ & 120 & 173 & 89 & 382 & 48 & 118 \\
\hline PRINCETON & bG+ & 143 & 225 & 42 & 410 & 37 & 116 \\
\hline PURDUE & G+ & 84 & 110 & 61 & 255 & 19 & 115 \\
\hline QUEENS & $\mathrm{LM}+$ & 45 & 106 & 18 & 169 & 14 & 115 \\
\hline RICE & G+ & 57 & 53 & 12 & 122 & 12 & 143 \\
\hline ROCHESTER & $\mathrm{bM}+$ & 95 & 61 & 57 & 213 & 17 & 119 \\
\hline RUTGERS & BGL+ & 100 & 238 & 81 & 419 & 34 & 108 \\
\hline SASKATCHEWAN & GLM+ & 52 & 88 & 11 & 151 & 13 & 90 \\
\hline SOUTH CAROLINA & $\mathrm{LM}+$ & 74 & 105 & 99 & 278 & 20 & 140 \\
\hline SOUTHERN CALIFORNIA & BGLM+ & 129 & 133 & 112 & 374 & 28 & 159 \\
\hline SOUTHERN ILLINOIS & $\mathrm{LM}+$ & 54 & 92 & 56 & 202 & 14 & 102 \\
\hline SUNY-ALBANY & G+ & 61 & 61 & 31 & 153 & 10 & 108 \\
\hline SUNY-BUFFALO & GLM+ & 106 & 66 & 78 & 250 & 15 & 168 \\
\hline SUNY-STONY BROOK & $\mathrm{M}+$ & 90 & 26 & 31 & 147 & 13 & 109 \\
\hline SYRACUSE & GL+ & 63 & 121 & 32 & 216 & 18 & 106 \\
\hline TEMPLE & BbGLM+ & 73 & 91 & 45 & 209 & 17 & 108 \\
\hline TENNESSEE & BbGLM+ & 86 & 133 & 49 & 268 & 17 & 148 \\
\hline TEXAS & GL+ & 141 & 285 & 102 & 528 & 34 & 120 \\
\hline TEXAS A\&M & $\mathrm{BbG}+$ & 145 & 132 & 82 & 359 & 18 & 146 \\
\hline TEXAS TECH & $\mathrm{BLM}+$ & 105 & 127 & 100 & 332 & 28 & 130 \\
\hline TORONTO & $\mathrm{B}+$ & 184 & 350 & 139 & 673 & 82 & 130 \\
\hline TULANE & $\mathrm{BLM}+$ & 45 & 76 & 26 & 147 & 20 & 118 \\
\hline UTAH & LM & 86 & 187 & 97 & 370 & 20 & 123 \\
\hline VANDERBILT & $\mathrm{LM}+$ & 106 & 105 & 30 & 241 & 16 & 147 \\
\hline VIRGINIA & GLM+ & 101 & 210 & 68 & 379 & 33 & 149 \\
\hline VIRGINIA TECH & G & 38 & 90 & 31 & 159 & 9 & 102 \\
\hline WASHINGTON & GLM+ & 193 & 206 & 124 & 523 & 48 & 138 \\
\hline WASHINGTON STATE & BG+ & 57 & 84 & 35 & 176 & 19 & 100 \\
\hline WASHINGTON U.-ST. LOUIS & bGLM+ & 93 & 115 & 58 & 266 & 20 & 120 \\
\hline WATERLOO & G+ & 36 & 98 & 38 & 172 & 13 & 106 \\
\hline WAYNE STATE & + & 97 & 86 & 64 & 247 & 17 & 142 \\
\hline WESTERN ONTARIO & GL+ & 71 & 111 & 13 & 195 & 8 & 105 \\
\hline WISCONSIN & GLM+ & 229 & 166 & 158 & 553 & 45 & 148 \\
\hline YALE & $\mathrm{LM}+$ & 272 & 396 & 67 & 735 & 42 & 111 \\
\hline YORK & BGL+ & 62 & 112 & 57 & 231 & 20 & 155 \\
\hline BOSTON PUBLIC & G & 154 & 304 & $\mathrm{UA} / \mathrm{NA}$ & 458 & 96 & 168 \\
\hline CISTI & + & 190 & 139 & 4 & 333 & 1 & 40 \\
\hline CENTER FOR RESEARCH LIBRARIES & + & 30 & 18 & 13 & 61 & 2 & 35 \\
\hline LIBRARY OF CONGRESS & bG+ & 3,294 & 343 & 50 & 3,687 & 38 & 65 \\
\hline
\end{tabular}




\section{Personnel and Public Services}

Library Participants Reference Initial Total Total Items Total Items Presentations in Group Transactions* Circulation Circulation Loaned Borrowed to Groups* Presentations* Transactions Transactions (ILL) (ILL)
(29)
(30)
(31)
(32)
(33)
(34)
(35) (Survey Question Number)

INSTITUTION

\begin{tabular}{|c|c|c|c|c|c|c|c|}
\hline 1,441 & 22,758 & 134,523 & 342,950 & 481,766 & 62,936 & 27,305 & PITTSBURGH \\
\hline 656 & 7,470 & 22,644 & 294,084 & 391,536 & 54,031 & 37,211 & PRINCETON \\
\hline 404 & 25,302 & 44,560 & 139,737 & 340,046 & 26,430 & 43,940 & PURDUE \\
\hline 756 & 16,288 & 39,841 & 218,296 & 495,052 & 20,064 & 9,722 & QUEENS \\
\hline 183 & 1,854 & UA/NA & 134,578 & 227,011 & 12,332 & 9,908 & RICE \\
\hline 609 & 8,816 & 52,229 & 212,176 & 239,623 & 27,420 & 23,921 & ROCHESTER \\
\hline 976 & 20,113 & 88,359 & 272,145 & 595,314 & 43,575 & 35,952 & RUTGERS \\
\hline 526 & 8,453 & 19,699 & 198,266 & 277,400 & 5,670 & 4,771 & SASKATCHEWAN \\
\hline 698 & 15,786 & 148,446 & 187,325 & 310,215 & 19,696 & 18,538 & SOUTH CAROLINA \\
\hline 932 & 14,322 & 61,017 & 328,488 & 539,739 & 24,627 & 20,600 & SOUTHERN CALIFORNIA \\
\hline 804 & 7,211 & 30,657 & 234,992 & 355,930 & 56,952 & 40,037 & SOUTHERN ILLINOIS \\
\hline 654 & 6,497 & 54,017 & 90,053 & 145,220 & 13,133 & 20,487 & SUNY-ALBANY \\
\hline 892 & 22,236 & 22,478 & 194,863 & 429,800 & 34,257 & 24,824 & SUNY-BUFFALO \\
\hline 563 & 10,189 & 43,641 & 197,092 & 208,857 & 20,820 & 13,198 & SUNY-STONY BROOK \\
\hline 542 & 11,271 & 52,926 & 150,273 & 206,451 & 13,775 & 21,580 & SYRACUSE \\
\hline 660 & 16,047 & 58,288 & 219,083 & 309,356 & 27,341 & 21,460 & TEMPLE \\
\hline 860 & 15,686 & 93,312 & 266,162 & 330,398 & 35,585 & 25,179 & TENNESSEE \\
\hline 605 & 60,003 & 131,492 & $2,105,396$ & $2,903,181$ & 57,999 & 27,822 & TEXAS \\
\hline 712 & 27,079 & 45,750 & $1,044,727$ & $1,690,414$ & 45,798 & 51,805 & TEXAS A\&M \\
\hline 1,463 & 16,069 & 51,540 & 242,731 & 346,963 & 41,739 & 33,051 & TEXAS TECH \\
\hline 3,686 & 56,607 & 534,328 & $1,240,474$ & $1,750,318$ & 28,195 & 8,409 & TORONTO \\
\hline 207 & 3,042 & 14,735 & 110,468 & 156,794 & 16,272 & 10,284 & TULANE \\
\hline 1,868 & 37,484 & 191,370 & 176,385 & 447,253 & 48,592 & 33,349 & UTAH \\
\hline 778 & 20,227 & 27,973 & 212,664 & 350,065 & 25,849 & 20,273 & VANDERBILT \\
\hline 1,110 & 10,940 & 115,433 & 490,068 & 871,544 & 39,945 & 30,606 & VIRGINIA \\
\hline 840 & 15,765 & 17,711 & 245,517 & 346,346 & 22,192 & 21,936 & VIRGINIA TECH \\
\hline 1,221 & 27,158 & 135,749 & 711,833 & $1,625,120$ & 86,879 & 92,703 & WASHINGTON \\
\hline 750 & 15,274 & 42,636 & 171,821 & 363,812 & 55,958 & 30,936 & WASHINGTON STATE \\
\hline 1,226 & 11,010 & 73,301 & 237,804 & 501,355 & 50,554 & 48,729 & WASHINGTON U.-ST. LOUIS \\
\hline 170 & 4,664 & 24,626 & 214,121 & 600,246 & 56,757 & 47,160 & WATERLOO \\
\hline 463 & 9,691 & 45,799 & 126,204 & 180,054 & 42,491 & 48,058 & WAYNE STATE \\
\hline 743 & 26,312 & 35,386 & 412,645 & 693,343 & 16,881 & 8,087 & WESTERN ONTARIO \\
\hline 2,692 & 35,037 & 2,640 & 521,843 & 896,335 & 92,683 & 88,726 & WISCONSIN \\
\hline 1,776 & 15,313 & 86,881 & 586,121 & 931,308 & 48,357 & 41,321 & YALE \\
\hline 856 & 26,616 & 132,174 & 554,909 & $1,074,780$ & 13,665 & 5,233 & YORK \\
\hline 11,269 & 182,275 & $1,090,826$ & $3,116,540$ & $3,116,540$ & 18,925 & 15,832 & BOSTON PUBLIC \\
\hline 485 & 485 & 22,940 & 12,033 & 29,201 & 441,772 & 17,230 & CISTI \\
\hline 43 & 1,706 & UA/NA & 93,853 & 93,853 & 29,939 & 4 & CENTER FOR RESEARCH LIBRARIES \\
\hline 6,212 & 210,609 & $1,740,490$ & $1,287,767$ & $1,287,767$ & 30,186 & 0 & LIBRARY OF CONGRESS \\
\hline
\end{tabular}




\section{Personnel and Public Services}

Professional Staff Support Staff Student Assistants Total Staff (FTE)

(FTE)

(FTE)

(FTE)

Staffed

Service

Library

Points Hours

(Survey Question Number)

(26.a)

(26.b)

(26.c)

(26)

(27)

(28)

INSTITUTION

Notes

\begin{tabular}{|c|c|c|c|c|c|c|c|}
\hline NATIONAL AGRICULTURAL LIBRARY & $\mathrm{bG}+$ & 130 & 66 & 0 & 196 & 13 & 40 \\
\hline LIBRARY \& ARCHIVES CANADA & G+ & 683 & 412 & 25 & 1,120 & 9 & 35 \\
\hline NATIONAL LIBRARY OF MEDICINE & G+ & 203 & 82 & 13 & 298 & 5 & 48 \\
\hline NEW YORK PUBLIC & + & 245 & 306 & 124 & 675 & 42 & 46 \\
\hline NEW YORK STATE & G+ & 49 & 89 & 5 & 143 & 5 & 40 \\
\hline SMITHSONIAN & G+ & 57 & 42 & 0 & 99 & 32 & 40 \\
\hline
\end{tabular}

+ - See Footnotes

L - Includes Law library

G - Government documents not included in serials count

B - Includes branch campuses

$\mathrm{b}$ - Basis of volume count is bibliographic
U/A - Unavailable

N/A - Not Applicable

- no data supplied

\section{Summary Data}

Professional Staff Support Staff Student Assistants Total Staff

(FTE)

(FTE)

(26.a)

(26.b)

(Survey Question Number)

UNIVERSITY LIBRARIES

Mean

Median

High

Low

Totals

Number of Libraries Reporting

NonUniversity Libraries

Median

Totals

Number of Libraries Reporting

GRAND TOTALS

$\begin{array}{rr}100 & 140 \\ 84 & 116 \\ 555 & 541 \\ 36 & 26 \\ 11,279 & 15,795 \\ 113 & 113\end{array}$

16,314

17,596

7,293

41,203
(FTE)

(FTE)

(26.c)

63
52
204
1
7,059
112

302

Staffed

Service

Points

(26)

(27)

(28)

Library Service Hours

122

258

1,229

117

34,133

113

2,624

112

13,697

112

$\begin{array}{rrrrrr}172 & 114 & 13 & 316 & 11 & 40 \\ 5,035 & 1,801 & 234 & 7,070 & 243 & 557 \\ 10 & 10 & 9 & 10 & 10 & 10\end{array}$

2,867

14,254 


\section{Personnel and Public Services}

$\begin{array}{rrr}\text { Library } & \text { Participants } & \text { Reference } \\ \begin{array}{r}\text { Presentations } \\ \text { to Groups* }\end{array} & \text { in Group } & \text { Transactions* }\end{array}$

(29)

(30)

(31)
Initial Circulation Transactions
Total Circulation Transactions otal Items

Loaned

(ILL)
Total Items

Borrowed

(ILL)

$\begin{array}{rrrrrrrr}\text { INSTITUTION } \\ 154 & 30,972 & 13,095 & 3,406 & 4,662 & 14,399 & 2,749 & \text { NATIONAL AGRICULTURAL LIBRARY } \\ 7 & \mathrm{UA} / \mathrm{NA} & 26,548 & \mathrm{UA} / \mathrm{NA} & 249,415 & 36,175 & 1,194 & \text { LIBRARY \& ARCHIVES CANADA } \\ 394 & 9,227 & 8,142 & 171,455 & 171,455 & 234,020 & 157 & \text { NATIONAL LIBRARY OF MEDICINE } \\ 561 & 7,689 & 615,986 & \mathrm{UA} / \mathrm{NA} & \mathrm{UA} / \mathrm{NA} & 2,243 & 7,279 & \text { NEW YORK PUBLIC } \\ 59 & 1,431 & 79,732 & 30,255 & 33,923 & 32,055 & 732 & \text { NEW YORK STATE } \\ 874 & 3,886 & 54,132 & 43,236 & 241,630 & 5,621 & 6,149 & \text { SMITHSONIAN }\end{array}$

*Figures in italics are derived from a sampling method rather than an actual count.

\section{Summary Data}

Library

Presentations

to Groups
Participants Reference in Group Transactions Presentations
Total Total Items Circulation Loaned (ILL) Transactions
Total Items

Borrowed

(ILL)
(29)

(30)

(31)

(32)

(33)

(34)

(35) (Survey Question Number)

UNIVERSITY LIBRARIES

$\begin{array}{rrrrrrrr}990 & 17,815 & 78,631 & 327,684 & 581,516 & 39,792 & 31,068 & \text { Mean } \\ 813 & 15,686 & 61,210 & 248,979 & 423,602 & 36,383 & 28,342 & \text { Median } \\ 3,686 & 60,003 & 534,328 & 2,105,396 & 2,903,181 & 170,711 & 96,771 & \text { High } \\ 170 & 1,854 & 2,640 & 90,053 & 105,790 & 5,670 & 4,771 & \text { Low } \\ 109,892 & 1,977,420 & 8,570,764 & 36,372,880 & 65,711,337 & 4,496,461 & 3,510,687 & \text { Totals } \\ 111 & 111 & 109 & 111 & 113 & 113 & 113 & \text { Number of Libraries Reporting }\end{array}$

20,058

440

$$
\text { 7,689 }
$$

54,132

68,545

171,455

30,063

1,972

$4,758,545$

$5,228,446$

845,335

51,326

10

10

NonUniVersity LibRARIES

Median

Totals

Number of Libraries Reporting 


\section{Analysis of Selected Variables of University Libraries, 2007-2008}

The percentages and ratios below are select indicators that describe the condition of ARL university libraries. The high and low figures indicate the range, while the mean and median indicate the central tendency of the distributions for ARL university libraries. Note that a distribution is normal when the mean and the median figures are identical. If the mean is larger than the median then the distribution is positively skewed, and if the opposite is true the distribution is negatively skewed.

Category

Professional Staff as a percent of Total Staff

Support Staff as a percent of Total Staff

Student Assistant Staff as a percent of Total Staff

Ratio of Professional to Support Staff (excluding Student Assistant Staff)

Ratio of Items Loaned to Items Borrowed

Serials Expenditures as a percent of Total Library Materials Expenditures

Monograph Expenditures as a percent of Total Library Materials Expenditures

Total Library Materials Expenditures as a percent of Total Library Expenditures

Contract Binding as a percent of Total Library Expenditures

Salary \& Wages Expenditures as a percent of Total Library Expenditures

Other Operating Expenditures as a percent of Total Library Expenditures

Unit costs of Monographs (per volume)

Unit costs of Serials (per title)

Library Expenditures per Faculty

Library Expenditures per Full-Time Student

Library Expenditures per Full-Time Graduate Student

Library Expenditures per Full-Time User

Library Expenditures per User

Library Expenditures per PhD Awarded

Library Staff per PhD Awarded

Professional Library Staff per PhD Awarded

High Mean Median

32.87

31.16

19.72

12.68

0.47

0.35

0.20

113

90.92

68.04

69.75

16.67

113

43.44

21.76

20.59

5.92

65.31

43.05

43.10

25.59

113

1.86

0.56

0.51

0.02

112

60.20

44.53

44.48

29.09

27.30

11.87

11.11

0.62

113

117.04

455.08

59.81

177.59

$55,054.07$

7,225.89

$18,165.07$

$1,365.22$

19,083.87

$5,671.21$

$6,216.23$

$1,253.63$

$6,131.54$

$339,587.55$

1,050.02

$95,564.59$

4.79

1.50
1.06

0.34
59.00

160.11

$16,395.68$

$1,028.28$

$5,168.77$

957.08

802.35

$80,789.74$

0.90

0.29
13.02

53.73

7,066.98

467.61

$2,436.36$

442.81

371.97

21,807.01

0.26

0.09

113 113 112 113 3 3 13 13 
PhD, Faculty, and Enrollment Statistics *

ENROLLMENTS

PhDs Awarded PhD Fields Faculty Total FTE Full-time ** Total Part-time** Graduate FTE Graduate
(Survey Question Number)
(36)
(37)
(38)
(39)
(40)
(41)

(42)

INSTITUTION

\begin{tabular}{|c|c|c|c|c|c|c|c|}
\hline ALABAMA & 154 & 44 & 913 & 21,642 & 3,289 & 2,281 & 1,589 \\
\hline ALBERTA & 341 & 116 & 1,588 & 33,412 & 3,536 & 4,935 & 1,434 \\
\hline ARIZONA & 388 & 85 & 1,582 & 31,058 & 6,159 & 6,289 & 2,322 \\
\hline ARIZONA STATE & 369 & 59 & 2,410 & 49,441 & 14,953 & 7,980 & 5,103 \\
\hline AUBURN & 196 & 52 & 1,132 & 20,542 & 3,595 & 1,564 & 1,811 \\
\hline BOSTON & 290 & 60 & 2,736 & 25,789 & 6,264 & 8,583 & 4,737 \\
\hline BOSTON COLLEGE & 154 & 36 & 675 & 11,507 & 2,607 & 2,406 & 2,236 \\
\hline BRIGHAM YOUNG & 80 & 24 & 1,305 & 27,844 & 3,029 & 1,943 & 1,358 \\
\hline BRITISH COLUMBIA & 359 & 91 & 2,504 & 34,052 & 15,525 & 7,177 & 1,704 \\
\hline BROWN & 205 & 43 & 701 & 7,850 & 317 & 2,060 & 99 \\
\hline CALIFORNIA BERKELEY & 865 & 89 & 1,501 & 32,742 & 1,438 & 8,879 & 665 \\
\hline CALIFORNIA DAVIS & 484 & 70 & 1,963 & 29,169 & 1,516 & 6,601 & 606 \\
\hline CALIFORNIA IRVINE & 370 & 45 & 1,490 & 26,077 & 1,049 & 4,979 & 451 \\
\hline CALIFORNIA LOS ANGELES & 752 & 84 & 2,654 & 37,566 & 1,330 & 10,639 & 311 \\
\hline CALIFORNIA RIVERSIDE & 224 & 42 & 826 & 16,733 & 454 & 2,177 & 37 \\
\hline CALIFORNIA SAN DIEGO & -1 & 72 & 1,644 & 26,861 & 827 & 5,535 & 105 \\
\hline CALIFORNIA SANTA BARBARA & 346 & 46 & 983 & 20,933 & 477 & 2,973 & 22 \\
\hline CASE WESTERN RESERVE & 205 & 57 & 601 & 7,588 & 2,256 & 3,592 & 2,045 \\
\hline CHICAGO & 395 & 66 & 1,633 & 11,853 & 2,685 & 6,996 & 2,616 \\
\hline CINCINNATI & 316 & 60 & 1,209 & 22,298 & 7,021 & 5,349 & 3,469 \\
\hline COLORADO & 301 & 45 & 1,259 & 25,959 & 5,837 & 2,000 & 3,448 \\
\hline COLORADO STATE & 206 & 43 & 974 & 21,885 & 5,684 & 2,527 & 3,371 \\
\hline COLUMBIA & 505 & 105 & 2,116 & 24,558 & 5,439 & 15,813 & 4,553 \\
\hline CONNECTICUT & 339 & 69 & 1,766 & 28,677 & 5,347 & 4,777 & 3,054 \\
\hline CORNELL & 476 & 83 & 1,728 & 19,763 & 37 & 6,268 & 22 \\
\hline DARTMOUTH & 70 & 15 & 777 & 5,746 & 103 & 1,637 & 48 \\
\hline DELAWARE & 208 & 39 & 1,117 & 17,679 & 2,663 & 2,514 & 891 \\
\hline DUKE & 352 & 44 & 990 & 13,005 & 297 & 6,744 & 283 \\
\hline EMORY & 212 & 42 & 1,807 & 11,681 & 889 & 5,040 & 811 \\
\hline FLORIDA & 858 & 124 & 2,020 & 40,914 & 5,849 & 10,246 & 3,792 \\
\hline FLORIDA STATE & 732 & 72 & 1,912 & 34,137 & 6,865 & 4,635 & 3,235 \\
\hline GEORGE WASHINGTON & 106 & 27 & 1,162 & 15,769 & 9,309 & 7,689 & 8,706 \\
\hline GEORGETOWN & 100 & 36 & 845 & 13,072 & 2,246 & 6,266 & 1,960 \\
\hline GEORGIA & 391 & 73 & 1,733 & 29,273 & 4,558 & 4,325 & 2,622 \\
\hline GEORGIA TECH & 467 & 48 & 863 & 16,832 & 1,910 & 5,103 & 1,074 \\
\hline GUELPH & 110 & (1) & 840 & 17,503 & 1,685 & 2,101 & 186 \\
\hline HARVARD & 534 & 74 & 2,517 & 19,513 & 6,177 & 12,377 & 3,454 \\
\hline HAWAII & 192 & 51 & 1,319 & 13,832 & 2,203 & 3,139 & 1,031 \\
\hline HOUSTON & 262 & 36 & 1,293 & 25,551 & 10,553 & 4,920 & 2,384 \\
\hline HOWARD & 101 & 29 & 1,091 & 9,643 & 1,090 & 3,426 & 615 \\
\hline ILLINOIS CHICAGO & 572 & 118 & 1,157 & 17,039 & 4,664 & 3,361 & 3,343 \\
\hline ILLINOIS URBANA & 759 & 91 & 2,309 & 40,399 & 1,156 & 10,195 & 672 \\
\hline
\end{tabular}


PhD, Faculty, and Enrollment Statistics *

ENROLLMENTS

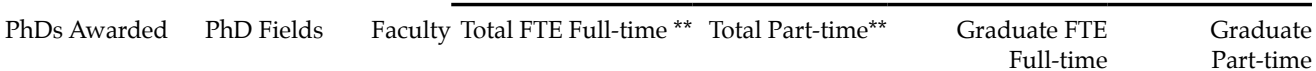
(Survey Question Number)
(36)
(37)
(38)
(39)
(40)
(41)

(42)

INSTITUTION

\begin{tabular}{|c|c|c|c|c|c|c|c|}
\hline INDIANA & 370 & 83 & 2,006 & 34,426 & 4,564 & 6,336 & 2,960 \\
\hline IOWA & 358 & 67 & 1,312 & 23,558 & 5,570 & 4,889 & 3,332 \\
\hline IOWA STATE & 308 & 82 & 1,346 & 22,762 & 3,398 & 2,600 & 2,064 \\
\hline JOHNS HOPKINS & 424 & 58 & 1,371 & 11,963 & 8,017 & 6,595 & 7,680 \\
\hline KANSAS & 327 & 76 & 1,724 & 22,797 & 5,772 & 4,226 & 3,515 \\
\hline KENT STATE & 115 & 59 & 881 & 17,754 & 5,065 & 2,329 & 2,400 \\
\hline KENTUCKY & 308 & 56 & 1,268 & 22,187 & 3,669 & 5,408 & 1,678 \\
\hline LAVAL & 297 & 66 & 1,454 & 25,519 & 11,535 & 5,283 & 4,308 \\
\hline LOUISIANA STATE & 237 & 55 & 1,438 & 27,019 & 2,789 & 4,100 & 1,203 \\
\hline LOUISVILLE & 135 & 30 & 1,550 & 17,214 & 5,628 & 6,564 & \\
\hline MCGILL & 394 & 10 & 3,442 & 32,410 & 5,798 & 10,177 & 1,901 \\
\hline MCMASTER & 231 & 54 & 1,772 & 25,172 & 4,089 & 3,517 & 724 \\
\hline MANITOBA & 98 & 47 & 1,341 & 21,254 & 5,080 & 2,487 & 803 \\
\hline MARYLAND & 655 & 70 & 3,502 & 30,594 & 5,390 & 6,844 & 3,313 \\
\hline MASSACHUSETTS & 250 & 50 & 1,180 & 21,055 & 5,305 & 2,058 & 3,762 \\
\hline MIT & 599 & 36 & 967 & 9,956 & 264 & 5,837 & 211 \\
\hline MIAMI & 116 & 41 & 920 & 14,209 & 1,240 & 4,532 & 538 \\
\hline MICHIGAN & 710 & 118 & 3,751 & 38,096 & 2,946 & 12,917 & 2,042 \\
\hline MICHIGAN STATE & 458 & 112 & 1,983 & 41,962 & 5,644 & 8,476 & 2,793 \\
\hline MINNESOTA & 775 & 100 & 1,763 & 36,448 & 14,395 & 9,397 & 9,192 \\
\hline MISSOURI & 286 & 65 & 1,206 & 23,448 & 4,292 & 3,164 & 2,990 \\
\hline MONTREAL & 378 & 86 & 1,903 & 38,417 & 16,257 & 10,762 & 3,990 \\
\hline NEBRASKA & 255 & 41 & 1,223 & 19,428 & 3,520 & 2,671 & 2,224 \\
\hline NEW MEXICO & 185 & 42 & 1,814 & 18,449 & 7,948 & 2,591 & 2,346 \\
\hline NEW YORK & 432 & 74 & 3,615 & 31,848 & 9,935 & 11,934 & 8,522 \\
\hline NORTH CAROLINA & 600 & 69 & 1,600 & 23,788 & 4,779 & 6,738 & 3,934 \\
\hline NORTH CAROLINA STATE & 328 & 56 & 1,723 & 24,989 & 6,813 & 4,265 & 3,392 \\
\hline NORTHWESTERN & 438 & 59 & 2,936 & 15,781 & 3,240 & 7,531 & 2,113 \\
\hline NOTRE DAME & 185 & 23 & 890 & 11,609 & 124 & 3,250 & 112 \\
\hline $\mathrm{OHIO}$ & 162 & 57 & 1,074 & 23,276 & 3,965 & 2,571 & 786 \\
\hline OHIO STATE & 759 & 103 & 3,047 & 52,336 & 8,011 & 10,495 & 3,162 \\
\hline OKLAHOMA & 192 & 57 & 2,117 & 24,046 & 6,090 & 5,715 & 3,061 \\
\hline OKLAHOMA STATE & 129 & 42 & 1,159 & 22,654 & 10,359 & 1,515 & 2,847 \\
\hline OREGON & 123 & 42 & 815 & 16,870 & 3,506 & 2,909 & 786 \\
\hline PENNSYLVANIA & 450 & 75 & 1,395 & 19,816 & 4,164 & 9,653 & 2,450 \\
\hline PENNSYLVANIA STATE & 658 & 117 & 5,466 & 70,005 & 9,993 & 6,900 & 3,542 \\
\hline PITTSBURGH & 392 & 68 & 4,224 & 29,496 & 4,989 & 7,174 & 2,961 \\
\hline PRINCETON & 307 & 57 & 825 & 7,225 & (1) & 2,380 & (1) \\
\hline PURDUE & 595 & 57 & 2,097 & 35,551 & 3,553 & 5,861 & 2,055 \\
\hline QUEENS & 140 & 38 & 834 & 17,217 & 3,499 & 3,135 & 380 \\
\hline RICE & 186 & 30 & 611 & 5,036 & 125 & 2,051 & 93 \\
\hline ROCHESTER & 220 & 44 & 1,944 & 7,744 & 1,590 & 2,905 & 1,298 \\
\hline
\end{tabular}


PhD, Faculty, and Enrollment Statistics *

ENROLLMENTS

$\begin{array}{lrrr}\text { PhDs Awarded PhD Fields Faculty Total FTE Full-time ** } & \text { Total Part-time** } & \begin{array}{r}\text { Graduate FTE } \\ \text { Full-time }\end{array} & \begin{array}{r}\text { Graduate } \\ \text { Part-time }\end{array}\end{array}$
(Survey Question Number)
(36)
(37)
(38)
(39)
(40)
(41)

(42)

INSTITUTION

\begin{tabular}{|c|c|c|c|c|c|c|c|}
\hline RUTGERS & 377 & 56 & 2,167 & 39,272 & 11,244 & 6,275 & 7,037 \\
\hline SASKATCHEWAN & 104 & 55 & 1,026 & 15,270 & 4,368 & 2,188 & 299 \\
\hline SOUTH CAROLINA & 251 & 61 & 1,705 & 21,969 & 5,303 & 4,722 & 3,723 \\
\hline SOUTHERN CALIFORNIA & 442 & 82 & 3,108 & 29,124 & 4,284 & 13,440 & 3,584 \\
\hline SOUTHERN ILLINOIS & 141 & 36 & 915 & 16,898 & 4,085 & 2,409 & 2,381 \\
\hline SUNY-ALBANY & 150 & 39 & 672 & 14,800 & 3,402 & 2,411 & 2,545 \\
\hline SUNY-BUFFALO & 394 & 76 & 1,193 & 23,579 & 4,475 & 6,062 & 3,213 \\
\hline SUNY-STONY BROOK & 306 & 38 & 919 & 16,629 & 3,430 & 3,205 & 2,434 \\
\hline SYRACUSE & 142 & 45 & 978 & 16,417 & 2,667 & 3,926 & 1,955 \\
\hline TEMPLE & 414 & 53 & 1,750 & 27,679 & 7,365 & 4,887 & 4,304 \\
\hline TENNESSEE & 355 & 72 & 1,576 & 26,195 & 3,741 & 6,302 & 2,265 \\
\hline TEXAS & 890 & 82 & 2,687 & 45,599 & 4,571 & 10,988 & 1,723 \\
\hline TEXAS A\&M & 594 & 92 & 2,345 & 43,493 & 5,493 & 7,643 & 2,096 \\
\hline TEXAS TECH & 198 & 56 & 1,781 & 26,195 & 4,276 & 4,495 & 1,744 \\
\hline TORONTO & 698 & 98 & 2,676 & 62,202 & 8,368 & 11,539 & 1,969 \\
\hline TULANE & 132 & 44 & 653 & 8,369 & 1,702 & 2,547 & 460 \\
\hline UTAH & 281 & 55 & 1,248 & 19,452 & 8,573 & 3,958 & 1,654 \\
\hline VANDERBILT & 253 & 46 & 2,541 & 11,319 & 774 & 4,735 & 721 \\
\hline VIRGINIA & 377 & 46 & 1,172 & 20,493 & 3,764 & 6,231 & 2,948 \\
\hline VIRGINIA TECH & 341 & 53 & 1,371 & 27,539 & 3,200 & 4,462 & 2,710 \\
\hline WASHINGTON & 631 & 80 & 3,728 & 36,365 & 8,379 & 9,603 & 2,989 \\
\hline WASHINGTON STATE & 189 & 45 & 1,178 & 20,169 & 4,227 & 2,065 & 1,265 \\
\hline WASHINGTON U.-ST. LOUIS & 235 & 56 & 784 & 11,010 & 2,372 & 4,869 & 1,260 \\
\hline WATERLOO & 193 & 43 & 915 & 22,459 & 1,883 & 3,023 & 611 \\
\hline WAYNE STATE & 214 & 59 & 1,775 & 18,468 & 13,912 & 5,823 & 5,412 \\
\hline WESTERN ONTARIO & 217 & 50 & 1,357 & 24,745 & 3,418 & 4,063 & 461 \\
\hline WISCONSIN & 755 & 107 & 2,054 & 37,224 & 4,817 & 9,345 & 2,078 \\
\hline YALE & 358 & 60 & 1,831 & 11,273 & 181 & 5,984 & 159 \\
\hline YORK & 149 & 29 & 1,520 & 42,279 & 9,540 & 3,720 & 2,022 \\
\hline
\end{tabular}

\section{Summary Data}

\begin{tabular}{lrrrrrrr} 
& (Survey Question Number) & $(36)$ & $(37)$ & $(38)$ & $(39)$ & $(40)$ & $(41)$ \\
\hline Median & 308 & 57 & 1,454 & 22,654 & 4,196 & 4,889 & 2,078 \\
High & 890 & 124 & 5,466 & 70,005 & 16,257 & 15,813 \\
Low & 70 & 10 & 601 & 5,036 & 37 & 1,515 & 2,192 \\
Totals & 38,692 & 6,791 & 186,759 & $2,729,256$ & 529,612 & 619,703 \\
Number of Libraries Reporting & 112 & 112 & 113 & 113 & 112 & 113
\end{tabular}

* - Figures were reported on the ARL questionnaire and have not been verified with the US NCES IPEDS survey.

** - Includes both undergraduate and graduate students.

UA/NA - Unavailable or Not Applicable 

RANK ORder TABles OF UNIVERSITy Libraries

2007-2008 
The table below presents the rank for each university library in each of the 21 categories for which rank order tables are prepared. The table numbers in the chart below refer to the data categories listed below. The number of libraries indicates the number of ARL university libraries supplying data in each category.

\begin{tabular}{llr} 
Table & Data Category & Number of Libraries \\
\hline & & \\
1 & VOLUMES IN LIBRARY & 113 \\
2 & VOLUMES ADDED (GROSS) & 113 \\
3 & CURRENT SERIALS (TOTAL) & 113 \\
4 & MICROFORM UNITS & 110 \\
5 & GOVERNMENT DOCUMENTS & 100 \\
6 & TOTAL LIBRARY MATERIALS EXPENDITURES & 113 \\
7 & TOTAL SALARIES \& WAGES EXPENDITURES & 113 \\
8 & OTHER OPERATING EXPENDITURES & 113 \\
9 & TOTAL LIBRARY EXPENDITURES & 113 \\
10 & MONOGRAPHS PURCHASED (VOLUMES) & 102 \\
11 & EXPENDITURES FOR MONOGRAPHS & 113 \\
12 & CURRENT SERIALS PURCHASED (TITLES) & 107 \\
13 & EXPENDITURES FOR CURRENT SERIALS & 113 \\
14 & TOTAL ITEMS LOANED (ILL/DD) & 113 \\
15 & TOTAL ITEMS BORROWED (ILL/DD) & 113 \\
16 & PROFESSIONAL STAFF (FTE) & 113 \\
17 & SUPPORT STAFF (FTE) & 113 \\
18 & TOTAL STAFF (FTE) & 113 \\
19 & EXPENDITURES FOR ELECTRONIC RESOURCES & 111 \\
20 & ELECTRONIC RESOURCES AS A \% OF TOTAL LIBRARY MATERIALS & 111 \\
21 & LIBRARY INVESTMENT INDEX & 113
\end{tabular}


\begin{tabular}{llllllllllllllllllllll} 
TABLE Number & 1 & 2 & 3 & 4 & 5 & 6 & 7 & 8 & 9 & 10 & 11 & 12 & 13 & 14 & 15 & 16 & 17 & 18 & 19 & 20 & 21 \\
\hline
\end{tabular}

\begin{tabular}{llllllllllllllllllllll}
\hline ALABAMA & 82 & 80 & 41 & 63 & 12 & 90 & 93 & 71 & 92 & 57 & 95 & 48 & 69 & 107 & 103 & 71 & 91 & 84 & 91 & 60 & 92
\end{tabular} ALBERTA $\begin{array}{llllllllllllllllllllll}16 & 11 & 84 & 74 & 68 & 12 & 18 & 3 & 10 & \text { UA } & 11 & 72 & 15 & 26 & 50 & 56 & 14 & 34 & 1 & 2 & 12\end{array}$

ARIZONA $\begin{array}{llllllllllllllllllllll}23 & 59 & 13 & 26 & \text { UA } & 34 & 39 & 15 & 32 & 48 & 35 & 4 & 38 & 23 & 35 & 46 & 37 & 39 & 20 & 31 & 32\end{array}$

$\begin{array}{lllllllllllllllllllllllll}\text { ARIZONA STATE } & 35 & 47 & 17 & 10 & 21 & 43 & 42 & 47 & 41 & 25 & 44 & 18 & 39 & 28 & 32 & 48 & 20 & 35 & 43 & 56 & 43\end{array}$
$\begin{array}{lllllllllllllllllllllll}\text { AUBURN } & 74 & 99 & 100 & 93 & 44 & 110 & 113 & 84 & 112 & 99 & 110 & 104 & 98 & 108 & 107 & 106 & 111 & 113 & 75 & 3 & 111\end{array}$ $\begin{array}{llllllllllllllllllllllllllll}\text { BOSTON } & 87 & 102 & 70 & 45 & \text { UA } & 66 & 66 & 65 & 69 & 97 & 87 & 39 & 45 & 89 & 80 & 38 & 76 & 46 & 101 & 98 & 69\end{array}$ \begin{tabular}{llllllllllllllllllllllllll}
\hline BOSTON COLLEGE & 98 & 95 & 106 & 59 & 46 & 72 & 70 & 98 & 78 & 59 & 82 & 97 & 95 & 70 & 74 & 63 & 96 & 84 & 72 & 53 & 75
\end{tabular} $\begin{array}{llllllllllllllllllllllllll}\text { BRIGHAM YOUNG } & 41 & 10 & 42 & 71 & 30 & 44 & 43 & 45 & 43 & 4 & 28 & 24 & 42 & 36 & 62 & 24 & 106 & 22 & 64 & 79 & 41\end{array}$ $\begin{array}{llllllllllllllllllllllllll}\text { BRITISH COLUMBIA } & 21 & 26 & 48 & 41 & 68 & 22 & 28 & 36 & 26 & \text { UA } & 26 & 19 & 13 & 82 & 71 & 29 & 26 & 33 & 16 & 64 & 25\end{array}$ $\begin{array}{lllllllllllllllllllllllll}\text { BROWN } & 46 & 44 & 61 & 103 & 68 & 81 & 67 & 68 & 75 & 31 & 62 & 59 & 87 & 49 & 28 & 66 & 73 & 79 & 79 & 54 & 74\end{array}$ $\begin{array}{llllllllllllllllllllllllllll}\text { CALIFORNIA BERKELEY } & 5 & 8 & 16 & 12 & 25 & 7 & 5 & 12 & 5 & \text { UA } & 4 & 6 & 12 & 43 & 76 & 4 & 19 & 7 & 32 & 104 & 5\end{array}$ $\begin{array}{lllllllllllllllllllllllllll}\text { CALIFORNIA DAVIS } & 52 & 41 & 65 & 56 & 52 & 86 & 53 & 82 & 72 & \text { UA } & 48 & 61 & 103 & 65 & 61 & 86 & 39 & 61 & 85 & 50 & 73\end{array}$ \begin{tabular}{llllllllllllllllllllllllllll}
\hline CALIFORNIA IRVINE & 75 & 51 & 82 & 91 & 50 & 65 & 60 & 56 & 63 & 51 & 37 & 81 & 65 & 72 & 45 & 66 & 50 & 62 & 74 & 69 & 63
\end{tabular} $\begin{array}{lllllllllllllllllllllllll}\text { CALIFORNIA LOS } & 11 & 27 & 29 & 28 & 53 & 21 & 6 & 5 & 6 & 18 & 7 & \text { UA } & 40 & 33 & 39 & 11 & 8 & 6 & 70 & 105 & 6\end{array}$ ANGELES

$\begin{array}{llllllllllllllllllllllll}\text { CALIFORNIA RIVERSIDE } & 96 & 87 & 11 & 99 & 38 & 107 & 84 & 93 & 100 & 74 & 90 & 12 & 104 & 94 & 95 & 104 & 92 & 101 & 108 & 91 & 101\end{array}$ $\begin{array}{lllllllllllllllllllllllll}\text { CALIFORNIA SAN DIEGO } & 64 & 31 & 80 & 82 & 68 & 62 & 27 & 33 & 34 & 40 & 40 & 82 & 64 & 52 & 56 & 35 & 22 & 29 & 62 & 43 & 34\end{array}$ $\begin{array}{llllllllllllllllllllllll}\text { CALIFORNIA SANTA } & 78 & 94 & 102 & 73 & 28 & 106 & 71 & 19 & 73 & 63 & 65 & 89 & 109 & 80 & 33 & 105 & 45 & 70 & 105 & 76 & 79\end{array}$ BARBARA

$\begin{array}{llllllllllllllllllllllll}\text { CASE WESTERN RESERVE } & 89 & 110 & 46 & 95 & 58 & 100 & 106 & 104 & 107 & 100 & 108 & 25 & 86 & 27 & 38 & 87 & 110 & 109 & 99 & 61 & 106\end{array}$ $\begin{array}{llllllllllllllllllllllllll}\text { CHICAGO } & 10 & 3 & 27 & 85 & \text { UA } & 9 & 35 & 39 & 27 & 6 & 9 & 68 & 4 & 29 & 73 & 75 & 23 & 37 & 46 & 102 & 28\end{array}$ $\begin{array}{llllllllllllllllllllllll}\text { CINCINNATI } & 54 & 38 & 19 & 81 & 57 & 59 & 78 & 77 & 74 & 53 & 45 & 13 & 57 & 11 & 23 & 64 & 107 & 94 & 52 & 45 & 72\end{array}$ $\begin{array}{llllllllllllllllllllllllll}\text { COLORADO } & 44 & 35 & 73 & 14 & 10 & 51 & 72 & 87 & 66 & 55 & 53 & 67 & 54 & 16 & 52 & 87 & 64 & 72 & 28 & 24 & 68\end{array}$ $\begin{array}{lllllllllllllllllllllllll}\text { COLORADOSTATE } & 111 & 78 & 103 & 110 & \text { UA } & 101 & 102 & 58 & 99 & 70 & 86 & 98 & 102 & 8 & 6 & 102 & 108 & 110 & 87 & 14 & 99\end{array}$ $\begin{array}{llllllllllllllllllllllllll}\text { COLUMBIA } & 6 & 1 & 1 & 23 & 49 & 4 & 4 & 10 & 4 & 1 & 10 & 3 & 1 & 37 & 9 & 2 & 10 & 3 & 2 & 81 & 4\end{array}$ $\begin{array}{lllllllllllllllllllllllllll}\text { CONNECTICUT } & 45 & 105 & 57 & 54 & 68 & 71 & 29 & 66 & 45 & 88 & 103 & 84 & 59 & 50 & 22 & 53 & 98 & 80 & 56 & 23 & 45\end{array}$ $\begin{array}{llllllllllllllllllllllllll}\text { CORNELL } & 12 & 25 & 9 & 6 & 68 & 19 & 10 & 8 & 9 & \text { UA } & 6 & \text { UA } & 41 & 20 & 40 & 24 & 5 & 10 & 22 & 85 & 11\end{array}$ $\begin{array}{llllllllllllllllllllllllll}\text { DARTMOUTH } & 86 & 71 & 43 & 94 & 68 & 76 & 75 & 96 & 82 & 60 & 71 & 21 & 56 & 59 & 49 & 83 & 56 & 81 & 63 & 28 & 82\end{array}$ $\begin{array}{lllllllllllllllllllllllllll}\text { DELAWARE } & 88 & 96 & 107 & 80 & 29 & 77 & 83 & 75 & 84 & 65 & 51 & 100 & 81 & 97 & 70 & 87 & 68 & 88 & 53 & 18 & 83\end{array}$ $\begin{array}{llllllllllllllllllllllllll}\text { DUKE } & 22 & 34 & 52 & 55 & 5 & 14 & 26 & 34 & 23 & 12 & 13 & 49 & 19 & 63 & 51 & 8 & 40 & 30 & 13 & 73 & 20\end{array}$ $\begin{array}{llllllllllllllllllllllllllll}\text { EMORY } & 62 & 42 & 75 & 27 & 43 & 23 & 32 & 37 & 30 & 29 & 12 & 73 & 99 & 56 & 81 & 46 & 35 & 41 & 80 & 107 & 29\end{array}$ $\begin{array}{lllllllllllllllllllllllllllll}\text { FLORIDA } & 36 & 66 & 39 & 8 & 6 & 36 & 31 & 69 & 37 & 44 & 85 & 51 & 17 & 78 & 53 & 38 & 24 & 24 & 48 & 80 & 37\end{array}$ $\begin{array}{lllllllllllllllllllllllllll}\text { FLORIDA STATE } & 85 & 73 & 53 & 4 & 14 & 85 & 91 & 111 & 95 & 36 & 89 & 57 & 90 & 95 & 93 & 75 & 61 & 62 & 60 & 9 & 94\end{array}$ $\begin{array}{lllllllllllllllllllllllllll}\text { GEORGE WASHINGTON } & 108 & 77 & 25 & 89 & 68 & 57 & 62 & 51 & 58 & 77 & 41 & 17 & 75 & 66 & 11 & 57 & 53 & 44 & 107 & 110 & 57\end{array}$ $\begin{array}{lllllllllllllllllllllllll}\text { GEORGETOWN } & 76 & 36 & 58 & 65 & 60 & 47 & 47 & 55 & 46 & 43 & 43 & 36 & 46 & 53 & 66 & 44 & 57 & 57 & 88 & 95 & 46\end{array}$ $\begin{array}{llllllllllllllllllllllll}\text { GEORGIA } & 32 & 48 & 22 & 18 & 8 & 40 & 59 & 59 & 54 & 34 & 46 & 28 & 37 & 44 & 110 & 62 & 27 & 40 & 38 & 47 & 53\end{array}$ $\begin{array}{lllllllllllllllllllllll}\text { GEORGIA TECH } & 97 & 93 & 91 & 47 & 68 & 105 & 111 & 103 & 108 & 103 & 109 & 105 & 106 & 109 & 100 & 108 & 99 & 111 & 89 & 7 & 108\end{array}$ $\begin{array}{lllllllllllllllllllllllll}\text { GUELPH } & 113 & 113 & 113 & 98 & 68 & 108 & 98 & 102 & 106 & 93 & 78 & 107 & 111 & 39 & 10 & 95 & 101 & 108 & 97 & 12 & 107\end{array}$ $\begin{array}{lllllllllllllllllllllllll}\text { HARVARD } & 1 & 2 & 3 & 2 & \text { UA } & 1 & 1 & 1 & 1 & \text { UA } & 1 & \text { UA } & 26 & 41 & 98 & 1 & 1 & 1 & 11 & 109 & 1\end{array}$ $\begin{array}{llllllllllllllllllllllll}\text { HAWAII } & 58 & 81 & 71 & 78 & 68 & 92 & 68 & 97 & 86 & 84 & 81 & 64 & 85 & 112 & 44 & 50 & 93 & 66 & 100 & 71 & 86\end{array}$ $\begin{array}{llllllllllllllllllllllllll}\text { HOUSTON } & 92 & 65 & 34 & 32 & 19 & 74 & 104 & 27 & 79 & 76 & 67 & 56 & 70 & 76 & 88 & 80 & 83 & 87 & 76 & 62 & 81\end{array}$ $\begin{array}{llllllllllllllllllllll}\text { HOWARD } & 100 & 112 & 109 & 57 & 62 & 113 & 108 & 106 & 113 & 104 & 113 & 101 & 112 & 110 & 111 & 103 & 102 & 95 & 110 & 49 & 113\end{array}$ $\begin{array}{lllllllllllllllllllllllllll}\text { ILLINOIS CHICAGO } & 101 & 37 & 92 & 58 & 15 & 69 & 81 & 64 & 80 & 46 & 88 & 69 & 105 & 14 & 8 & 69 & 72 & 83 & 77 & 66 & 78\end{array}$ $\begin{array}{llllllllllllllllllllllllll}\text { ILLINOIS URBANA } & 3 & 7 & 4 & 3 & 68 & 28 & 12 & 30 & 18 & 11 & 33 & 2 & 22 & 3 & 5 & 6 & 21 & 13 & 45 & 89 & 18\end{array}$ $\begin{array}{lllllllllllllllllllllllll}\text { INDIANA } & 9 & 16 & 63 & 16 & 35 & 15 & 21 & 22 & 19 & 9 & 8 & 45 & 31 & 32 & 19 & 10 & 28 & 14 & 31 & 90 & 19\end{array}$ $\begin{array}{lllllllllllllllllllllllllll}\text { IOWA } & 30 & 12 & 66 & \text { UA } & \text { UA } & 30 & 40 & 86 & 39 & 15 & 47 & 62 & 18 & 12 & 55 & 42 & 52 & 49 & 39 & 77 & 38\end{array}$ $\begin{array}{llllllllllllllllllllllll}\text { IOWA STATE } & 95 & 91 & 47 & 77 & 68 & 67 & 89 & 80 & 83 & 62 & 69 & 52 & 55 & 68 & 90 & 99 & 86 & 97 & 36 & 5 & 84\end{array}$ 
\begin{tabular}{llllllllllllllllllllll} 
TABLE Number & 1 & 2 & 3 & 4 & 5 & 6 & 7 & 8 & 9 & 10 & 11 & 12 & 13 & 14 & 15 & 16 & 17 & 18 & 19 & 20 & 21 \\
\hline
\end{tabular} \begin{tabular}{llllllllllllllllllllllll}
\hline JOHNS HOPKINS & 47 & 101 & 6 & 52 & 66 & 26 & 51 & 42 & 35 & 72 & 58 & 9 & 10 & 55 & 31 & 21 & 43 & 38 & 71 & 101 & 36
\end{tabular} $\begin{array}{lllllllllllllllllllllllll}\text { KANSAS } & 37 & 72 & 54 & 75 & 18 & 63 & 55 & 61 & 62 & 26 & 56 & 46 & 79 & 31 & 30 & 36 & 65 & 48 & 67 & 63 & 62\end{array}$ $\begin{array}{llllllllllllllllllllll}\text { KENT STATE } & 84 & 74 & 62 & \text { UA } & 68 & 112 & 112 & 53 & 111 & 98 & 106 & 23 & 113 & 102 & 106 & 93 & 112 & 47 & \text { UA } & \text { UA } & 112\end{array}$ $\begin{array}{llllllllllllllllllllllllll}\text { KENTUCKY } & 49 & 45 & 32 & 21 & 9 & 56 & 77 & 57 & 65 & 41 & 75 & 80 & 66 & 67 & 67 & 51 & 44 & 44 & 41 & 30 & 66\end{array}$ $\begin{array}{lllllllllllllllllllllllll}\text { LAVAL } & 80 & 57 & 101 & 109 & 68 & 58 & 57 & 112 & 68 & 45 & 39 & 88 & 51 & 96 & 105 & 83 & 35 & 74 & 50 & 44 & 67\end{array}$ $\begin{array}{llllllllllllllllllllllll}\text { LOUISIANA STATE } & 40 & 100 & 7 & 5 & 23 & 91 & 103 & 94 & 102 & 54 & 60 & 5 & 93 & 111 & 91 & 99 & 73 & 91 & 86 & 33 & 102\end{array}$ $\begin{array}{lllllllllllllllllllllllll}\text { LOUISVILLE } & 110 & 60 & 79 & 101 & \text { UA } & 68 & 101 & 46 & 81 & 69 & 104 & 33 & 100 & 81 & 85 & 98 & 78 & 93 & 102 & 99 & 80\end{array}$ $\begin{array}{lllllllllllllllllllllll}\text { MCGILL } & 34 & 43 & 30 & 104 & 16 & 18 & 30 & 31 & 25 & 2 & 14 & 22 & 14 & 86 & 92 & 59 & 31 & 42 & 8 & 42 & 26\end{array}$ $\begin{array}{llllllllllllllllllllllll}\text { MCMASTER } & 106 & 14 & 77 & 106 & 68 & 83 & 85 & 91 & 87 & 56 & 101 & 66 & 83 & 100 & 99 & 108 & 78 & 100 & 61 & 13 & 88\end{array}$ $\begin{array}{lllllllllllllllllllllllll}\text { MANITOBA } & 105 & 64 & 98 & 108 & 45 & 82 & 46 & 76 & 64 & 30 & 57 & 92 & 80 & 88 & 63 & 78 & 38 & 67 & 84 & 58 & 65\end{array}$ $\begin{array}{llllllllllllllllllllllll}\text { MARYLAND } & 50 & 33 & 95 & 33 & 47 & 60 & 36 & 73 & 51 & 50 & 76 & 95 & 48 & 83 & 27 & 28 & 78 & 57 & 35 & 16 & 49\end{array}$ $\begin{array}{llllllllllllllllllllllll}\text { MASSACHUSETTS } & 56 & 83 & 93 & 96 & 68 & 102 & 88 & 78 & 94 & 91 & 100 & 86 & 97 & 40 & 16 & 93 & 96 & 92 & 83 & 6 & 95\end{array}$ $\begin{array}{llllllllllllllllllllllllll}\text { MIT } & 79 & 55 & 78 & 97 & \text { UA } & 75 & 34 & 49 & 50 & 79 & 74 & 74 & 60 & 93 & 89 & 36 & 81 & 74 & 58 & 26 & 50\end{array}$ $\begin{array}{lllllllllllllllllllllllll}\text { MIAMI } & 66 & 53 & 31 & 66 & 68 & 32 & 63 & 32 & 42 & 37 & 30 & 16 & 21 & 48 & 96 & 60 & 46 & 51 & 24 & 59 & 42\end{array}$ $\begin{array}{llllllllllllllllllllllll}\text { MICHIGAN } & 8 & 20 & 40 & \mathrm{UA} & 68 & 6 & 7 & 25 & 7 & 8 & 15 & \mathrm{UA} & 2 & 15 & 21 & 11 & 6 & 8 & 15 & 94 & 7\end{array}$ $\begin{array}{lllllllllllllllllllllllll}\text { MICHIGAN STATE } & 29 & 46 & 8 & 17 & \text { UA } & 46 & 48 & 60 & 47 & 24 & 50 & 11 & 35 & 13 & 47 & 60 & 54 & 43 & 37 & 37 & 47\end{array}$ $\begin{array}{lllllllllllllllllllllllll}\text { MINNESOTA } & 17 & 28 & 20 & 13 & 2 & 13 & 19 & 16 & 17 & 39 & 23 & 29 & 5 & 2 & 24 & 30 & 18 & 21 & 12 & 72 & 17\end{array}$ $\begin{array}{lllllllllllllllllllllllll}\text { MISSOURI } & 59 & 89 & 99 & 7 & 3 & 80 & 100 & 81 & 91 & 73 & 102 & 94 & 77 & 24 & 26 & 87 & 61 & 88 & 40 & 4 & 91\end{array}$ $\begin{array}{llllllllllllllllllllllll}\text { MONTREAL } & 70 & 67 & 64 & 107 & 68 & 45 & 20 & 101 & 33 & 28 & 61 & 37 & 28 & 85 & 86 & 31 & 9 & 28 & 27 & 29 & 33\end{array}$ $\begin{array}{lllllllllllllllllllllll}\text { NEBRASKA } & 69 & 88 & 94 & 46 & 24 & 95 & 92 & 92 & 96 & 101 & 111 & 77 & 67 & 62 & 54 & 95 & 66 & 82 & 106 & 100 & 96\end{array}$ $\begin{array}{llllllllllllllllllllllll} & \text { NEW MEXICO } & 90 & 84 & 38 & 44 & 22 & 78 & 44 & 48 & 55 & 87 & 80 & 30 & 62 & 87 & 60 & 71 & 40 & 54 & 59 & 20 & 59\end{array}$

$\begin{array}{lllllllllllllllllllllll}\text { NEW YORK } & 28 & 32 & 44 & 35 & 27 & 16 & 14 & 11 & 12 & 21 & 19 & 58 & 8 & 64 & 36 & 19 & 12 & 16 & 6 & 39 & 13\end{array}$
$\begin{array}{lllllllllllllllllllllll}\text { NORTH CAROLINA } & 18 & 24 & 59 & 42 & 4 & 17 & 16 & 17 & 15 & 10 & 29 & 76 & 24 & 17 & 94 & 14 & 16 & 17 & 65 & 106 & 15\end{array}$ $\begin{array}{lllllllllllllllllllllllll}\text { NORTH CAROLINA STATE } & 42 & 23 & 55 & 38 & 68 & 61 & 50 & 9 & 38 & 61 & 64 & 85 & 61 & 101 & 72 & 23 & 68 & 52 & 94 & 93 & 39\end{array}$ $\begin{array}{lllllllllllllllllllllll}\text { NORTHWESTERN } & 31 & 52 & 24 & 49 & 61 & 29 & 38 & 54 & 36 & 47 & 22 & 54 & 30 & 57 & 57 & 24 & 42 & 32 & 21 & 57 & 35\end{array}$ $\begin{array}{llllllllllllllllllllllll}\text { NOTRE DAME } & 61 & 54 & 21 & 67 & 68 & 39 & 54 & 70 & 56 & 32 & 17 & 20 & 63 & 69 & 68 & 71 & 34 & 56 & 33 & 38 & 54\end{array}$ $\begin{array}{lllllllllllllllllllllll}\text { OHIO } & 81 & 62 & 96 & 102 & \text { UA } & 109 & 107 & 100 & 109 & 66 & 91 & 91 & 110 & 46 & 14 & 106 & 99 & 103 & 103 & 65 & 110\end{array}$ $\begin{array}{lllllllllllllllllllllllll}\text { OHIO STATE } & 19 & 18 & 12 & 30 & 67 & 33 & 25 & 6 & 20 & 13 & 27 & 8 & 32 & 1 & 1 & 16 & 33 & 14 & 30 & 68 & 22\end{array}$ $\begin{array}{lllllllllllllllllllllllll}\text { OKLAHOMA } & 27 & 4 & 51 & 51 & 56 & 27 & 96 & 74 & 60 & 58 & 25 & 35 & 33 & 60 & 43 & 83 & 76 & 68 & 78 & 103 & 58\end{array}$ $\begin{array}{llllllllllllllllllllllllll}\text { OKLAHOMA STATE } & 83 & 58 & 76 & 50 & 48 & 98 & 82 & 67 & 90 & 80 & 79 & 60 & 92 & 54 & 79 & 77 & 94 & 76 & \text { UA } & \text { UA } & 90\end{array}$ $\begin{array}{llllllllllllllllllllllllll}\text { OREGON } & 77 & 69 & 88 & 61 & 41 & 99 & 86 & 72 & 93 & 81 & 72 & 75 & 108 & 6 & 7 & 71 & 88 & 71 & 104 & 88 & 93\end{array}$ $\begin{array}{lllllllllllllllllllllllll}\text { PENNSYLVANIA } & 20 & 29 & 33 & 62 & \text { UA } & 20 & 23 & 18 & 22 & \text { UA } & 21 & 10 & 16 & 7 & 4 & 20 & 29 & 20 & 25 & 83 & 21\end{array}$ $\begin{array}{lllllllllllllllllllllllll}\text { PENNSYLVANIA STATE } & 26 & 13 & 14 & 15 & 68 & 8 & 8 & 23 & 8 & 16 & 20 & 7 & 3 & 9 & 12 & 13 & 3 & 5 & 3 & 32 & 8\end{array}$ $\begin{array}{llllllllllllllllllllllllll}\text { PITSBURGH } & 24 & 19 & 67 & 39 & 68 & 25 & 37 & 35 & 31 & \text { UA } & 36 & 41 & 27 & 10 & 59 & 24 & 30 & 23 & 4 & 11 & 30\end{array}$ $\begin{array}{lllllllllllllllllllllllll}\text { PRINCETON } & 15 & 17 & 81 & 20 & 63 & 5 & 17 & 24 & 11 & 7 & 5 & 40 & 11 & 25 & 34 & 16 & 13 & 19 & 10 & 92 & 10\end{array}$ $\begin{array}{llllllllllllllllllllllll}\text { PURDUE } & 99 & 90 & 97 & 86 & 26 & 54 & 49 & 38 & 48 & 92 & 77 & 87 & 58 & 75 & 20 & 57 & 61 & 59 & 34 & 22 & 48\end{array}$ $\begin{array}{llllllllllllllllllllllll}\text { QUEENS } & 94 & 92 & 72 & 69 & 7 & 64 & 76 & 89 & 77 & 67 & 73 & 47 & 50 & 91 & 104 & 108 & 66 & 99 & 49 & 27 & 76\end{array}$ $\begin{array}{lllllllllllllllllllllll}\text { RICE } & 93 & 82 & 37 & 84 & 55 & 49 & 110 & 110 & 89 & 42 & 18 & 26 & 73 & 106 & 102 & 91 & 109 & 112 & 73 & 87 & 87\end{array}$ $\begin{array}{lllllllllllllllllllllllllll}\text { ROCHESTER } & 51 & 98 & 108 & 43 & 31 & 89 & 69 & 13 & 59 & 82 & 98 & 96 & 72 & 73 & 69 & 44 & 104 & 76 & 98 & 82 & 61\end{array}$ $\begin{array}{lllllllllllllllllllllllll}\text { RUTGERS } & 33 & 50 & 35 & 31 & 1 & 55 & 15 & 63 & 29 & 83 & 84 & 44 & 47 & 42 & 37 & 41 & 11 & 18 & 66 & 75 & 31\end{array}$ $\begin{array}{lllllllllllllllllllllll}\text { SASKATCHEWAN } & 112 & 107 & 105 & 87 & 32 & 73 & 87 & 99 & 85 & 85 & 55 & 99 & 68 & 113 & 113 & 99 & 86 & 105 & 54 & 19 & 85\end{array}$ $\begin{array}{lllllllllllllllllllllllll}\text { SOUTH CAROLINA } & 55 & 85 & 49 & 40 & 11 & 87 & 79 & 44 & 76 & 90 & 83 & 103 & 74 & 92 & 87 & 65 & 68 & 50 & 69 & 21 & 77\end{array}$ $\begin{array}{lllllllllllllllllllllllll}\text { SOUTHERN CALIFORNIA } & 39 & 70 & 5 & 24 & 40 & 48 & 22 & 4 & 21 & 23 & 34 & 14 & 44 & 79 & 82 & 22 & 46 & 26 & 44 & 55 & 23\end{array}$ $\begin{array}{llllllllllllllllllllllllll} & \\ \text { SOUTHERN ILLINOIS } & 73 & 103 & 86 & 48 & 37 & 97 & 95 & 90 & 98 & 94 & 107 & 71 & 78 & 19 & 29 & 95 & 82 & 84 & 93 & 40 & 97\end{array}$ $\begin{array}{llllllllllllllllllllll}\text { SUNY-ALBANY } & 109 & 39 & 74 & 90 & 68 & 111 & 97 & 113 & 110 & 95 & 105 & 50 & 107 & 105 & 83 & 82 & 104 & 103 & 111 & 96 & 109\end{array}$ 


\begin{tabular}{|c|c|c|c|c|c|c|c|c|c|c|c|c|c|c|c|c|c|c|c|c|c|}
\hline TABLe Number & 1 & 2 & 3 & 4 & 5 & 6 & 7 & 8 & 9 & 10 & 11 & 12 & 13 & 14 & 15 & 16 & 17 & 18 & 19 & 20 & 21 \\
\hline SUNY-BUFFALO & 48 & 49 & 23 & 29 & 68 & 79 & 52 & 83 & 71 & 75 & 92 & 27 & 53 & 61 & 65 & 32 & 103 & 60 & 55 & 15 & 70 \\
\hline SUNY-STONY BROOK & 107 & 111 & 50 & 72 & 36 & 93 & 99 & 108 & 103 & 102 & 112 & 32 & 94 & 90 & 97 & 52 & 113 & 106 & 92 & 41 & 100 \\
\hline SYRACUSE & 71 & 109 & 104 & 11 & 39 & 96 & 74 & 85 & 88 & 86 & 94 & 93 & 91 & 103 & 77 & 79 & 55 & 72 & 95 & 51 & 89 \\
\hline TEMPLE & 68 & 61 & 60 & 83 & 59 & 41 & 80 & 50 & 61 & 35 & 52 & 55 & 84 & 74 & 78 & 66 & 83 & 78 & 29 & 35 & 60 \\
\hline TENNESSEE & 65 & 63 & 83 & 53 & 65 & 37 & 61 & 95 & 57 & 49 & 54 & 79 & 25 & 58 & 64 & 53 & 46 & 52 & 51 & 78 & 55 \\
\hline TEXAS & 7 & 15 & 69 & 19 & 68 & 10 & 13 & 21 & 13 & 33 & 24 & 70 & 20 & 18 & 58 & 18 & 7 & 11 & 9 & 74 & 9 \\
\hline TEXAS A\&M & 43 & 9 & 18 & 34 & 54 & 11 & 41 & 26 & 28 & 14 & 16 & 34 & 9 & 38 & 13 & 15 & 49 & 31 & 7 & 52 & 27 \\
\hline TEXAS TECH & 91 & 76 & 45 & 92 & 64 & 50 & 64 & 29 & 49 & 22 & 38 & 38 & 52 & 47 & 42 & 34 & 50 & 35 & 68 & 84 & 52 \\
\hline TORONTO & 4 & 5 & 28 & 37 & UA & 3 & 3 & 14 & 3 & $b$ & 3 & 43 & 6 & 71 & 108 & 9 & 4 & 4 & 5 & 97 & 3 \\
\hline TULANE & 67 & 30 & 36 & 100 & 51 & 84 & 109 & 109 & 104 & 38 & 66 & 53 & 76 & 99 & 101 & 108 & 94 & 106 & 81 & 46 & 104 \\
\hline UTAH & 63 & 75 & 85 & 76 & 13 & 88 & 33 & 43 & 53 & UA & 63 & 90 & 82 & 34 & 41 & 53 & 25 & 27 & 109 & 108 & 56 \\
\hline VANDERBILT & 60 & 68 & 87 & 88 & 42 & 42 & 58 & 52 & 52 & 64 & 68 & 83 & 23 & 77 & 84 & 32 & 68 & 65 & 17 & 8 & 51 \\
\hline VIRGINIA & 25 & 40 & 10 & 36 & 20 & 31 & 24 & 20 & 24 & 19 & 31 & 63 & 36 & 51 & 48 & 40 & 15 & 25 & 47 & 86 & 24 \\
\hline VIRGINIA TECH & 102 & 106 & 112 & 25 & 33 & 94 & 105 & 105 & 105 & 89 & 99 & 106 & 89 & 84 & 75 & 112 & 85 & 101 & 82 & 17 & 105 \\
\hline WASHINGTON & 14 & 22 & 56 & 9 & 68 & 24 & 11 & 41 & 16 & 27 & 32 & UA & 7 & 5 & 2 & 7 & 17 & 12 & 18 & 67 & 16 \\
\hline WASHINGTON STATE & 103 & 104 & 110 & 68 & 68 & 104 & 94 & 88 & 101 & 96 & 97 & UA & 96 & 22 & 46 & 91 & 90 & 95 & 90 & 10 & 103 \\
\hline $\begin{array}{l}\text { WASHINGTON U.-ST. } \\
\text { LOUIS }\end{array}$ & 38 & 56 & 15 & 79 & 68 & 35 & 56 & 28 & 40 & 71 & 70 & 15 & 34 & 30 & 15 & 48 & 58 & 55 & 26 & 48 & 40 \\
\hline WATERLOO & 104 & 108 & 111 & 105 & 68 & 103 & 90 & 79 & 97 & 17 & 93 & 102 & 101 & 21 & 18 & 113 & 75 & 98 & 96 & 34 & 98 \\
\hline WAYNE STATE & 53 & 97 & 90 & 70 & 34 & 70 & 65 & 62 & 70 & 78 & 96 & 78 & 49 & 45 & 17 & 43 & 89 & 64 & 14 & 1 & 71 \\
\hline WESTERN ONTARIO & 57 & 86 & 68 & 64 & 68 & 38 & 73 & 107 & 67 & 68 & 49 & 31 & 29 & 98 & 109 & 69 & 60 & 90 & 23 & 25 & 64 \\
\hline WISCONSIN & 13 & 21 & 26 & 22 & 17 & 52 & 9 & 7 & 14 & 20 & 59 & 42 & 71 & 4 & 3 & 5 & 32 & 9 & 57 & 70 & 14 \\
\hline YALE & 2 & 6 & 2 & 1 & 68 & 2 & 2 & 2 & 2 & 0 & 2 & 1 & 88 & 35 & 25 & 3 & 2 & 2 & 19 & 111 & 2 \\
\hline YORK & 72 & 79 & 89 & 60 & UA & 53 & 45 & 40 & 44 & 52 & 42 & 65 & 43 & 104 & 112 & 80 & 59 & 69 & 42 & 36 & 44 \\
\hline
\end{tabular}

UA - Unavailable 
Rank Order Table 1: Volumes in Library

\begin{tabular}{|c|c|c|c|c|c|}
\hline RANK & INSTITUTION & Value & RANK & INSTITUTION & VALUE \\
\hline 1 & HARVARD & $16,250,117$ & 58 & HAWAII & $3,533,671$ \\
\hline 2 & YALE & $12,519,514$ & 59 & MISSOURI & $3,494,470$ \\
\hline 3 & ILLINOIS URBANA & $11,686,060$ & 60 & VANDERBILT & $3,440,317$ \\
\hline 4 & TORONTO & $11,186,488$ & 61 & NOTRE DAME & $3,393,290$ \\
\hline 5 & CALIFORNIA BERKELEY & $11,087,687$ & 62 & EMORY & $3,377,140$ \\
\hline 6 & COLUMBIA & $10,296,816$ & 63 & UTAH & $3,373,141$ \\
\hline 7 & TEXAS & $9,447,434$ & 64 & CALIFORNIA SAN DIEGO & $3,372,785$ \\
\hline 8 & MICHIGAN & $9,175,102$ & 65 & TENNESSEE & $3,289,447$ \\
\hline 9 & INDIANA & $8,723,253$ & 66 & MIAMI & $3,265,503$ \\
\hline 10 & CHICAGO & $8,597,159$ & 67 & TULANE & $3,257,609$ \\
\hline 11 & CALIFORNIA LOS ANGELES & $8,393,910$ & 68 & TEMPLE & $3,205,539$ \\
\hline 12 & CORNELL & $8,141,781$ & 69 & NEBRASKA & $3,204,060$ \\
\hline 13 & WISCONSIN & $8,059,335$ & 70 & MONTREAL & $3,183,053$ \\
\hline 14 & WASHINGTON & $7,409,221$ & 71 & SYRACUSE & $3,174,700$ \\
\hline 15 & PRINCETON & $6,941,254$ & 72 & YORK & $3,151,882$ \\
\hline 16 & ALBERTA & $6,890,679$ & 73 & SOUTHERN ILLINOIS & $3,099,151$ \\
\hline 17 & MINNESOTA & $6,877,699$ & 74 & AUBURN & $3,053,468$ \\
\hline 18 & NORTH CAROLINA & $6,526,824$ & 75 & CALIFORNIA IRVINE & $3,049,706$ \\
\hline 19 & OHIO STATE & $6,285,446$ & 76 & GEORGETOWN & $3,041,624$ \\
\hline 20 & PENNSYLVANIA & $6,096,592$ & 77 & OREGON & $2,959,739$ \\
\hline 21 & BRITISH COLUMBIA & $6,040,261$ & 78 & CALIFORNIASANTA BARBARA & $2,948,999$ \\
\hline 22 & DUKE & $5,950,442$ & 79 & MIT & $2,914,052$ \\
\hline 23 & ARIZONA & $5,722,280$ & 80 & LAVAL & $2,894,709$ \\
\hline 24 & PITTSBURGH & $5,657,403$ & 81 & OHIO & $2,893,238$ \\
\hline 25 & VIRGINIA & $5,532,266$ & 82 & ALABAMA & $2,892,166$ \\
\hline 26 & PENNSYLVANIA STATE & $5,354,645$ & 83 & OKLAHOMA STATE & $2,867,680$ \\
\hline 27 & OKLAHOMA & $5,299,924$ & 84 & KENT STATE & $2,853,965$ \\
\hline 28 & NEW YORK & $5,073,286$ & 85 & FLORIDA STATE & $2,844,624$ \\
\hline 29 & MICHIGAN STATE & $4,994,033$ & 86 & DARTMOUTH & $2,785,925$ \\
\hline 30 & IOWA & $4,949,636$ & 87 & BOSTON & $2,783,137$ \\
\hline 31 & NORTHWESTERN & $4,842,949$ & 88 & DELAWARE & $2,779,271$ \\
\hline 32 & GEORGIA & $4,637,291$ & 89 & CASE WESTERN RESERVE & $2,751,662$ \\
\hline 33 & RUTGERS & $4,529,396$ & 90 & NEW MEXICO & $2,737,747$ \\
\hline 34 & MCGILL & $4,498,389$ & 91 & TEXAS TECH & $2,617,844$ \\
\hline 35 & ARIZONA STATE & $4,422,239$ & 92 & HOUSTON & $2,617,282$ \\
\hline 36 & FLORIDA & $4,288,118$ & 93 & RICE & $2,570,768$ \\
\hline 37 & KANSAS & $4,235,542$ & 94 & QUEENS & $2,542,264$ \\
\hline 38 & WASHINGTON U.-ST. LOUIS & $4,224,113$ & 95 & IOWA STATE & $2,529,921$ \\
\hline 39 & SOUTHERN CALIFORNIA & $4,084,284$ & 96 & CALIFORNIA RIVERSIDE & $2,527,607$ \\
\hline 40 & LOUISIANA STATE & $4,082,803$ & 97 & GEORGIA TECH & $2,524,671$ \\
\hline 41 & BRIGHAM YOUNG & $4,080,079$ & 98 & BOSTON COLLEGE & $2,512,605$ \\
\hline 42 & NORTH CAROLINA STATE & $3,981,278$ & 99 & PURDUE & $2,509,158$ \\
\hline 43 & TEXAS A\&M & $3,933,535$ & 100 & HOWARD & $2,455,985$ \\
\hline 44 & COLORADO & $3,928,431$ & 101 & ILLINOIS CHICAGO & $2,447,459$ \\
\hline 45 & CONNECTICUT & $3,919,423$ & 102 & VIRGINIA TECH & $2,331,055$ \\
\hline 46 & BROWN & $3,825,413$ & 103 & WASHINGTON STATE & $2,326,652$ \\
\hline 47 & JOHNS HOPKINS & $3,740,029$ & 104 & WATERLOO & $2,286,415$ \\
\hline 48 & SUNY-BUFFALO & $3,720,113$ & 105 & MANITOBA & $2,284,284$ \\
\hline 49 & KENTUCKY & $3,719,548$ & 106 & MCMASTER & $2,280,435$ \\
\hline 50 & MARYLAND & $3,716,860$ & 107 & SUNY-STONY BROOK & $2,257,590$ \\
\hline 51 & ROCHESTER & $3,701,241$ & 108 & GEORGE WASHINGTON & $2,238,894$ \\
\hline 52 & CALIFORNIA DAVIS & $3,683,867$ & 109 & SUNY-ALBANY & $2,225,241$ \\
\hline 53 & WAYNE STATE & $3,660,642$ & 110 & LOUISVILLE & $2,191,091$ \\
\hline 54 & CINCINNATI & $3,631,658$ & 111 & COLORADO STATE & $2,045,603$ \\
\hline 55 & SOUTH CAROLINA & $3,629,954$ & 112 & SASKATCHEWAN & $2,029,016$ \\
\hline 56 & MASSACHUSETTS & $3,578,753$ & 113 & GUELPH & $1,853,628$ \\
\hline 57 & WESTERN ONTARIO & $3,546,496$ & & & \\
\hline
\end{tabular}


Rank Order Table 2: Volumes Added (Gross)

\begin{tabular}{|c|c|c|c|c|c|}
\hline RANK & INSTITUTION & VALUE & RANK & INSTITUTION & VALUE \\
\hline 1 & COLUMBIA & 547,346 & 58 & OKLAHOMA STATE & 71,378 \\
\hline 2 & HARVARD & 319,547 & 59 & ARIZONA & 69,004 \\
\hline 3 & CHICAGO & 277,507 & 60 & LOUISVILLE & 68,955 \\
\hline 4 & OKLAHOMA & 257,137 & 61 & TEMPLE & 68,903 \\
\hline 5 & TORONTO & 247,666 & 62 & OHIO & 67,904 \\
\hline 6 & YALE & 238,862 & 63 & TENNESSEE & 67,867 \\
\hline 7 & ILLINOIS URBANA & 208,659 & 64 & MANITOBA & 67,745 \\
\hline 8 & CALIFORNIA BERKELEY & 207,779 & 65 & HOUSTON & 66,505 \\
\hline 9 & TEXAS A\&M & 206,223 & 66 & FLORIDA & 65,257 \\
\hline 10 & BRIGHAM YOUNG & 187,005 & 67 & MONTREAL & 62,208 \\
\hline 11 & ALBERTA & 186,313 & 68 & VANDERBILT & 59,525 \\
\hline 12 & IOWA & 185,900 & 69 & OREGON & 58,993 \\
\hline 13 & PENNSYLVANIA STATE & 184,457 & 70 & SOUTHERN CALIFORNIA & 58,810 \\
\hline 14 & MCMASTER & 178,927 & 71 & DARTMOUTH & 58,147 \\
\hline 15 & TEXAS & 178,892 & 72 & KANSAS & 56,777 \\
\hline 16 & INDIANA & 174,417 & 73 & FLORIDA STATE & 55,821 \\
\hline 17 & PRINCETON & 174,320 & 74 & KENT STATE & 55,781 \\
\hline 18 & OHIO STATE & 161,118 & 75 & UTAH & 55,501 \\
\hline 19 & PITTSBURGH & 160,722 & 76 & TEXAS TECH & 54,715 \\
\hline 20 & MICHIGAN & 146,729 & 77 & GEORGE WASHINGTON & 54,595 \\
\hline 21 & WISCONSIN & 139,835 & 78 & COLORADO STATE & 53,932 \\
\hline 22 & WASHINGTON & 139,683 & 79 & YORK & 53,699 \\
\hline 23 & NORTH CAROLINA STATE & 126,853 & 80 & ALABAMA & 53,470 \\
\hline 24 & NORTH CAROLINA & 126,279 & 81 & HAWAII & 52,882 \\
\hline 25 & CORNELL & 126,058 & 82 & RICE & 52,881 \\
\hline 26 & BRITISH COLUMBIA & 122,887 & 83 & MASSACHUSETTS & 52,701 \\
\hline 27 & CALIFORNIA LOS ANGELES & 121,136 & 84 & NEW MEXICO & 51,768 \\
\hline 28 & MINNESOTA & 120,523 & 85 & SOUTH CAROLINA & 50,393 \\
\hline 29 & PENNSYLVANIA & 113,609 & 86 & WESTERN ONTARIO & 50,007 \\
\hline 30 & TULANE & 113,591 & 87 & CALIFORNIA RIVERSIDE & 49,797 \\
\hline 31 & CALIFORNIA SAN DIEGO & 108,073 & 88 & NEBRASKA & 49,588 \\
\hline 32 & NEW YORK & 107,390 & 89 & MISSOURI & 45,462 \\
\hline 33 & MARYLAND & 105,517 & 90 & PURDUE & 43,874 \\
\hline 34 & DUKE & 105,419 & 91 & IOWA STATE & 43,326 \\
\hline 35 & COLORADO & 98,800 & 92 & QUEEN'S & 42,383 \\
\hline 36 & GEORGETOWN & 98,442 & 93 & GEORGIA TECH & 41,514 \\
\hline 37 & ILLINOIS CHICAGO & 97,904 & 94 & CALIFORNIA SANTA BARBARA & 40,432 \\
\hline 38 & CINCINNATI & 95,810 & 95 & BOSTON COLLEGE & 40,325 \\
\hline 39 & SUNY-ALBANY & 92,459 & 96 & DELAWARE & 39,898 \\
\hline 40 & VIRGINIA & 91,904 & 97 & WAYNE STATE & 39,045 \\
\hline 41 & CALIFORNIA DAVIS & 91,692 & 98 & ROCHESTER & 38,465 \\
\hline 42 & EMORY & 89,560 & 99 & AUBURN & 38,068 \\
\hline 43 & MCGILL & 89,015 & 100 & LOUISIANA STATE & 37,946 \\
\hline 44 & BROWN & 87,499 & 101 & JOHNS HOPKINS & 37,223 \\
\hline 45 & KENTUCKY & 85,805 & 102 & BOSTON & 35,581 \\
\hline 46 & MICHIGAN STATE & 85,666 & 103 & SOUTHERN ILLINOIS & 35,178 \\
\hline 47 & ARIZONA STATE & 84,467 & 104 & WASHINGTON STATE & 35,166 \\
\hline 48 & GEORGIA & 83,468 & 105 & CONNECTICUT & 34,095 \\
\hline 49 & SUNY-BUFFALO & 82,074 & 106 & VIRGINIA TECH & 33,780 \\
\hline 50 & RUTGERS & 81,114 & 107 & SASKATCHEWAN & 31,988 \\
\hline 51 & CALIFORNIA IRVINE & 79,732 & 108 & WATERLOO & 30,818 \\
\hline 52 & NORTHWESTERN & 79,608 & 109 & SYRACUSE & 30,585 \\
\hline 53 & MIAMI & 76,116 & 110 & CASE WESTERN RESERVE & 26,072 \\
\hline 54 & NOTRE DAME & 75,659 & 111 & SUNY-STONY BROOK & 25,594 \\
\hline 55 & MIT & 75,469 & 112 & HOWARD & 24,428 \\
\hline 56 & WASHINGTON U.-ST. LOUIS & 74,408 & 113 & GUELPH & 23,025 \\
\hline 57 & LAVAL & 73,125 & & & \\
\hline
\end{tabular}


Rank Order Table 3: Current Serials (Total)

\begin{tabular}{|c|c|c|c|c|c|}
\hline RANK & INSTITUTION & VALUE & RANK & INSTITUTION & VALUE \\
\hline 1 & COLUMBIA & 133,831 & 58 & GEORGETOWN & 61,257 \\
\hline 2 & YALE & 110,802 & 59 & NORTH CAROLINA & 60,713 \\
\hline 3 & HARVARD & 110,628 & 60 & TEMPLE & 60,586 \\
\hline 4 & ILLINOIS URBANA & 110,299 & 61 & BROWN & 60,499 \\
\hline 5 & SOUTHERN CALIFORNIA & 105,744 & 62 & KENT STATE & 60,015 \\
\hline 6 & JOHNS HOPKINS & 105,453 & 63 & INDIANA & 59,924 \\
\hline 7 & LOUISIANA STATE & 101,738 & 64 & MONTREAL & 59,574 \\
\hline 8 & MICHIGAN STATE & 98,469 & 65 & CALIFORNIA DAVIS & 59,463 \\
\hline 9 & CORNELL & 98,000 & 66 & IOWA & 59,442 \\
\hline 10 & VIRGINIA & 97,800 & 67 & PITTSBURGH & 59,141 \\
\hline 11 & CALIFORNIA RIVERSIDE & 89,811 & 68 & WESTERN ONTARIO & 58,715 \\
\hline 12 & OHIO STATE & 89,239 & 69 & TEXAS & 56,991 \\
\hline 13 & ARIZONA & 88,737 & 70 & BOSTON & 56,113 \\
\hline 14 & PENNSYLVANIA STATE & 88,668 & 71 & HAWAII & 55,925 \\
\hline 15 & WASHINGTON U.-ST. LOUIS & 88,635 & 72 & QUEEN'S & 55,646 \\
\hline 16 & CALIFORNIA BERKELEY & 87,876 & 73 & COLORADO & 55,519 \\
\hline 17 & ARIZONA STATE & 87,566 & 74 & SUNY-ALBANY & 54,874 \\
\hline 18 & TEXAS A\&M & 86,737 & 75 & EMORY & 54,295 \\
\hline 19 & CINCINNATI & 86,363 & 76 & OKLAHOMA STATE & 53,799 \\
\hline 20 & MINNESOTA & 85,075 & 77 & MCMASTER & 53,584 \\
\hline 21 & NOTRE DAME & 82,866 & 78 & MIT & 53,548 \\
\hline 22 & GEORGIA & 80,748 & 79 & LOUISVILLE & 53,461 \\
\hline 23 & SUNY-BUFFALO & 80,431 & 80 & CALIFORNIA SAN DIEGO & 52,535 \\
\hline 24 & NORTHWESTERN & 77,933 & 81 & PRINCETON & 51,746 \\
\hline 25 & GEORGE WASHINGTON & 76,810 & 82 & CALIFORNIA IRVINE & 51,623 \\
\hline 26 & WISCONSIN & 76,808 & 83 & TENNESSEE & 50,552 \\
\hline 27 & CHICAGO & 76,607 & 84 & ALBERTA & 50,281 \\
\hline 28 & TORONTO & 76,473 & 85 & UTAH & 48,777 \\
\hline 29 & CALIFORNIA LOS ANGELES & 76,248 & 86 & SOUTHERN ILLINOIS & 47,871 \\
\hline 30 & MCGILL & 75,328 & 87 & VANDERBILT & 47,211 \\
\hline 31 & MIAMI & 74,775 & 88 & OREGON & 46,650 \\
\hline 32 & KENTUCKY & 73,251 & 89 & YORK & 46,539 \\
\hline 33 & PENNSYLVANIA & 73,248 & 90 & WAYNE STATE & 45,400 \\
\hline 34 & HOUSTON & 72,775 & 91 & GEORGIA TECH & 44,875 \\
\hline 35 & RUTGERS & 72,623 & 92 & ILLINOIS CHICAGO & 44,614 \\
\hline 36 & TULANE & 72,564 & 93 & MASSACHUSETTS & 43,906 \\
\hline 37 & RICE & 72,352 & 94 & NEBRASKA & 42,589 \\
\hline 38 & NEW MEXICO & 71,416 & 95 & MARYLAND & 42,393 \\
\hline 39 & FLORIDA & 71,336 & 96 & OHIO & 41,741 \\
\hline 40 & MICHIGAN & 69,457 & 97 & PURDUE & 40,094 \\
\hline 41 & ALABAMA & 69,426 & 98 & MANITOBA & 39,332 \\
\hline 42 & BRIGHAM YOUNG & 69,361 & 99 & MISSOURI & 38,364 \\
\hline 43 & DARTMOUTH & 68,661 & 100 & AUBURN & 37,980 \\
\hline 44 & NEW YORK & 67,960 & 101 & LAVAL & 37,701 \\
\hline 45 & TEXAS TECH & 66,750 & 102 & CALIFORNIASANTA BARBARA & 36,990 \\
\hline 46 & CASE WESTERN RESERVE & 66,625 & 103 & COLORADO STATE & 36,133 \\
\hline 47 & IOWA STATE & 66,195 & 104 & SYRACUSE & 34,443 \\
\hline 48 & BRITISH COLUMBIA & 64,669 & 105 & SASKATCHEWAN & 32,157 \\
\hline 49 & SOUTH CAROLINA & 63,715 & 106 & BOSTON COLLEGE & 31,465 \\
\hline 50 & SUNY-STONY BROOK & 63,423 & 107 & DELAWARE & 29,246 \\
\hline 51 & OKLAHOMA & 63,407 & 108 & ROCHESTER & 28,637 \\
\hline 52 & DUKE & 62,639 & 109 & HOWARD & 26,687 \\
\hline 53 & FLORIDA STATE & 62,093 & 110 & WASHINGTON STATE & 25,264 \\
\hline 54 & KANSAS & 62,016 & 111 & WATERLOO & 23,705 \\
\hline 55 & NORTH CAROLINA STATE & 61,990 & 112 & VIRGINIA TECH & 22,765 \\
\hline 56 & WASHINGTON & 61,979 & 113 & GUELPH & 11,986 \\
\hline 57 & CONNECTICUT & 61,812 & & & \\
\hline
\end{tabular}


Rank Order Table 4: Microform Units

\begin{tabular}{|c|c|c|c|c|c|}
\hline RANK & INSTITUTION & VAlue & RANK & INSTITUTION & VALUE \\
\hline 1 & YALE & $10,269,372$ & 58 & ILLINOIS CHICAGO & $4,197,939$ \\
\hline 2 & HARVARD & $9,814,796$ & 59 & BOSTON COLLEGE & $4,190,134$ \\
\hline 3 & ILLINOIS URBANA & $9,636,471$ & 60 & YORK & $4,187,860$ \\
\hline 4 & FLORIDA STATE & $9,109,694$ & 61 & OREGON & $4,159,767$ \\
\hline 5 & LOUISIANA STATE & $9,014,560$ & 62 & PENNSYLVANIA & $4,147,587$ \\
\hline 6 & CORNELL & $8,507,563$ & 63 & ALABAMA & $4,096,093$ \\
\hline 7 & MISSOURI & $8,118,672$ & 64 & WESTERN ONTARIO & $4,062,350$ \\
\hline 8 & FLORIDA & $7,901,639$ & 65 & GEORGETOWN & $4,026,834$ \\
\hline 9 & WASHINGTON & $7,788,178$ & 66 & MIAMI & $3,993,799$ \\
\hline 10 & ARIZONA STATE & $7,588,679$ & 67 & NOTRE DAME & $3,958,901$ \\
\hline 11 & SYRACUSE & $7,491,672$ & 68 & WASHINGTON STATE & $3,938,825$ \\
\hline 12 & CALIFORNIA BERKELEY & $7,128,372$ & 69 & QUEEN'S & $3,923,674$ \\
\hline 13 & MINNESOTA & $7,008,203$ & 70 & WAYNE STATE & $3,896,048$ \\
\hline 14 & COLORADO & $6,993,960$ & 71 & BRIGHAM YOUNG & $3,874,728$ \\
\hline 15 & PENNSYLVANIA STATE & $6,933,343$ & 72 & SUNY-STONY BROOK & $3,837,217$ \\
\hline 16 & INDIANA & $6,882,420$ & 73 & CALIFORNIASANTA BARBARA & $3,771,838$ \\
\hline 17 & MICHIGAN STATE & $6,776,737$ & 74 & ALBERTA & $3,738,979$ \\
\hline 18 & GEORGIA & $6,632,493$ & 75 & KANSAS & $3,657,049$ \\
\hline 19 & TEXAS & $6,628,794$ & 76 & UTAH & $3,638,146$ \\
\hline 20 & PRINCETON & $6,550,035$ & 77 & IOWA STATE & $3,521,259$ \\
\hline 21 & KENTUCKY & $6,471,766$ & 78 & HAWAII & $3,517,793$ \\
\hline 22 & WISCONSIN & $6,350,062$ & 79 & WASHINGTON U.-ST. LOUIS & $3,448,834$ \\
\hline 23 & COLUMBIA & $6,345,302$ & 80 & DELAWARE & $3,443,953$ \\
\hline 24 & SOUTHERN CALIFORNIA & $6,329,276$ & 81 & CINCINNATI & $3,371,800$ \\
\hline 25 & VIRGINIA TECH & $6,323,119$ & 82 & CALIFORNIA SAN DIEGO & $3,309,102$ \\
\hline 26 & ARIZONA & $6,282,673$ & 83 & TEMPLE & $3,308,050$ \\
\hline 27 & EMORY & $6,254,488$ & 84 & RICE & $3,276,520$ \\
\hline 28 & CALIFORNIA LOS ANGELES & $6,218,665$ & 85 & CHICAGO & $3,114,979$ \\
\hline 29 & SUNY-BUFFALO & $6,120,752$ & 86 & PURDUE & $3,104,959$ \\
\hline 30 & OHIO STATE & $6,059,429$ & 87 & SASKATCHEWAN & $3,087,965$ \\
\hline 31 & RUTGERS & $6,048,708$ & 88 & VANDERBILT & $3,044,268$ \\
\hline 32 & HOUSTON & $5,985,299$ & 89 & GEORGE WASHINGTON & $2,946,196$ \\
\hline 33 & MARYLAND & $5,832,845$ & 90 & SUNY-ALBANY & $2,896,185$ \\
\hline 34 & TEXAS A\&M & $5,686,474$ & 91 & CALIFORNIA IRVINE & $2,893,477$ \\
\hline 35 & NEW YORK & $5,561,308$ & 92 & TEXAS TECH & $2,726,735$ \\
\hline 36 & VIRGINIA & $5,536,709$ & 93 & AUBURN & $2,718,458$ \\
\hline 37 & TORONTO & $5,491,412$ & 94 & DARTMOUTH & $2,605,009$ \\
\hline 38 & NORTH CAROLINA STATE & $5,446,201$ & 95 & CASE WESTERN RESERVE & $2,601,135$ \\
\hline 39 & PITTSBURGH & $5,442,379$ & 96 & MASSACHUSETTS & $2,584,531$ \\
\hline 40 & SOUTH CAROLINA & $5,357,428$ & 97 & MIT & $2,416,804$ \\
\hline 41 & BRITISH COLUMBIA & $5,291,774$ & 98 & GUELPH & $2,346,336$ \\
\hline 42 & NORTH CAROLINA & $5,142,758$ & 99 & CALIFORNIA RIVERSIDE & $2,280,683$ \\
\hline 43 & ROCHESTER & $5,120,381$ & 100 & TULANE & $2,252,124$ \\
\hline 44 & NEW MEXICO & $4,721,312$ & 101 & LOUISVILLE & $2,219,718$ \\
\hline 45 & BOSTON & $4,700,124$ & 102 & OHIO & $2,000,195$ \\
\hline 46 & NEBRASKA & $4,687,095$ & 103 & BROWN & $1,966,000$ \\
\hline 47 & GEORGIA TECH & $4,627,573$ & 104 & MCGILL & $1,848,185$ \\
\hline 48 & SOUTHERN ILLINOIS & $4,601,724$ & 105 & WATERLOO & $1,710,239$ \\
\hline 49 & NORTHWESTERN & $4,554,601$ & 106 & MCMASTER & $1,599,937$ \\
\hline 50 & OKLAHOMA STATE & $4,533,703$ & 107 & MONTREAL & $1,547,142$ \\
\hline 51 & OKLAHOMA & $4,465,689$ & 108 & MANITOBA & $1,473,614$ \\
\hline 52 & JOHNS HOPKINS & $4,394,653$ & 109 & LAVAL & $1,299,734$ \\
\hline 53 & TENNESSEE & $4,389,259$ & 110 & COLORADO STATE & $1,159,032$ \\
\hline 54 & CONNECTICUT & $4,320,791$ & & IOWA & $\mathrm{UA} / \mathrm{NA}$ \\
\hline 55 & DUKE & $4,313,350$ & & KENT STATE & $\mathrm{UA} / \mathrm{NA}$ \\
\hline 56 & CALIFORNIA DAVIS & $4,241,335$ & & MICHIGAN & $\mathrm{UA} / \mathrm{NA}$ \\
\hline 57 & HOWARD & $4,234,651$ & & & \\
\hline
\end{tabular}


Rank Order Table 5: Government Documents

\begin{tabular}{|c|c|c|c|c|c|}
\hline RANK & INSTITUTION & Value & RANK & INSTITUTION & V \\
\hline 1 & RUTGERS & $2,668,634$ & 58 & CASE WESTERN RESERVE & 29,017 \\
\hline 2 & MINNESOTA & $2,640,164$ & 59 & TEMPLE & 24,766 \\
\hline 3 & MISSOURI & $1,681,883$ & 60 & GEORGETOWN & 9,385 \\
\hline 4 & NORTH CAROLINA & $1,625,932$ & 61 & NORTHWESTERN & 7,808 \\
\hline 5 & DUKE & $1,257,457$ & 62 & HOWARD & 6,759 \\
\hline 6 & FLORIDA & $1,241,342$ & 63 & PRINCETON & 4,646 \\
\hline 7 & QUEENS & $1,114,838$ & 64 & TEXAS TECH & 4,222 \\
\hline 8 & GEORGIA & $1,066,004$ & 65 & TENNESSEE & 4,158 \\
\hline 9 & KENTUCKY & $1,036,210$ & 66 & JOHNS HOPKINS & 1,324 \\
\hline 10 & COLORADO & 963,779 & 67 & OHIO STATE & 832 \\
\hline 11 & SOUTH CAROLINA & 944,768 & 68 & ALBERTA & 0 \\
\hline 12 & ALABAMA & 926,705 & 68 & BRITISH COLUMBIA & 0 \\
\hline 13 & UTAH & 898,343 & 68 & BROWN & 0 \\
\hline 14 & FLORIDA STATE & 857,378 & 68 & CALIFORNIA SAN DIEGO & 0 \\
\hline 15 & ILLINOIS CHICAGO & 793,177 & 68 & CONNECTICUT & 0 \\
\hline 16 & MCGILL & 784,146 & 68 & CORNELL & 0 \\
\hline 17 & WISCONSIN & 757,805 & 68 & DARTMOUTH & 0 \\
\hline 18 & KANSAS & 739,024 & 68 & GEORGE WASHINGTON & 0 \\
\hline 19 & HOUSTON & 680,971 & 68 & GEORGIA TECH & 0 \\
\hline 20 & VIRGINIA & 674,257 & 68 & GUELPH & 0 \\
\hline 21 & ARIZONA STATE & 664,596 & 68 & HAWAII & 0 \\
\hline 22 & NEW MEXICO & 663,556 & 68 & ILLINOIS URBANA & 0 \\
\hline 23 & LOUISIANA STATE & 590,485 & 68 & IOWA STATE & 0 \\
\hline 24 & NEBRASKA & 552,672 & 68 & KENT STATE & 0 \\
\hline 25 & CALIFORNIA BERKELEY & 492,722 & 68 & LAVAL & 0 \\
\hline 26 & PURDUE & 485,929 & 68 & MCMASTER & 0 \\
\hline 27 & NEW YORK & 482,172 & 68 & MASSACHUSETTS & 0 \\
\hline 28 & CALIFORNIA SANTA BARBARA & 451,767 & 68 & MIAMI & 0 \\
\hline 29 & DELAWARE & 451,186 & 68 & MICHIGAN & 0 \\
\hline 30 & BRIGHAM YOUNG & 423,514 & 68 & MONTREAL & 0 \\
\hline 31 & ROCHESTER & 419,912 & 68 & NORTH CAROLINA STATE & 0 \\
\hline 32 & SASKATCHEWAN & 414,618 & 68 & NOTRE DAME & 0 \\
\hline 33 & VIRGINIA TECH & 399,525 & 68 & PENNSYLVANIA STATE & 0 \\
\hline 34 & WAYNE STATE & 388,182 & 68 & PITTSBURGH & 0 \\
\hline 35 & INDIANA & 383,266 & 68 & SUNY-ALBANY & 0 \\
\hline 36 & SUNY-STONY BROOK & 347,884 & 68 & SUNY-BUFFALO & 0 \\
\hline 37 & SOUTHERN ILLINOIS & 312,699 & 68 & TEXAS & 0 \\
\hline 38 & CALIFORNIA RIVERSIDE & 299,797 & 68 & WASHINGTON & 0 \\
\hline 39 & SYRACUSE & 291,548 & 68 & WASHINGTON STATE & 0 \\
\hline 40 & SOUTHERN CALIFORNIA & 271,720 & 68 & WASHINGTON U.-ST. LOUIS & 0 \\
\hline 41 & OREGON & 268,640 & 68 & WATERLOO & 0 \\
\hline 42 & VANDERBILT & 263,092 & 68 & WESTERN ONTARIO & 0 \\
\hline 43 & EMORY & 256,899 & 68 & YALE & 0 \\
\hline 44 & AUBURN & 237,752 & & ARIZONA & $\mathrm{UA} / \mathrm{NA}$ \\
\hline 45 & MANITOBA & 222,243 & & BOSTON & $\mathrm{UA} / \mathrm{NA}$ \\
\hline 46 & BOSTON COLLEGE & 215,703 & & CHICAGO & $\mathrm{UA} / \mathrm{NA}$ \\
\hline 47 & MARYLAND & 199,706 & & COLORADO STATE & $\mathrm{UA} / \mathrm{NA}$ \\
\hline 48 & OKLAHOMA STATE & 180,337 & & HARVARD & $\mathrm{UA} / \mathrm{NA}$ \\
\hline 49 & COLUMBIA & 175,665 & & IOWA & $\mathrm{UA} / \mathrm{NA}$ \\
\hline 50 & CALIFORNIA IRVINE & 152,779 & & LOUISVILLE & $\mathrm{UA} / \mathrm{NA}$ \\
\hline 51 & TULANE & 94,550 & & MIT & $\mathrm{UA} / \mathrm{NA}$ \\
\hline 52 & CALIFORNIA DAVIS & 79,264 & & MICHIGAN STATE & UA/NA \\
\hline 53 & CALIFORNIA LOS ANGELES & 72,716 & & $\mathrm{OHIO}$ & $\mathrm{UA} / \mathrm{NA}$ \\
\hline 54 & TEXAS A\&M & 65,519 & & PENNSYLVANIA & $\mathrm{UA} / \mathrm{NA}$ \\
\hline 55 & RICE & 55,896 & & TORONTO & $\mathrm{UA} / \mathrm{NA}$ \\
\hline 56 & OKLAHOMA & 35,360 & & YORK & $\mathrm{UA} / \mathrm{NA}$ \\
\hline 57 & CINCINNATI & 30,140 & & & \\
\hline
\end{tabular}


Rank Order Table 6: Total Library Materials Expenditures

\begin{tabular}{|c|c|c|c|c|c|}
\hline RANK & INSTITUTION & VAlue & RANK & INSTITUTION & VALUE \\
\hline 1 & HARVARD & $34,291,329$ & 58 & LAVAL & $10,374,866$ \\
\hline 2 & YALE & $33,345,261$ & 59 & CINCINNATI & $10,283,685$ \\
\hline 3 & TORONTO & $25,822,409$ & 60 & MARYLAND & $10,097,607$ \\
\hline 4 & COLUMBIA & $23,685,056$ & 61 & NORTH CAROLINA STATE & $9,809,078$ \\
\hline 5 & PRINCETON & $21,197,104$ & 62 & CALIFORNIA SAN DIEGO & $9,583,762$ \\
\hline 6 & MICHIGAN & $20,525,876$ & 63 & KANSAS & $9,581,850$ \\
\hline 7 & CALIFORNIA BERKELEY & $20,118,847$ & 64 & QUEEN'S & $9,543,294$ \\
\hline 8 & PENNSYLVANIA STATE & $17,826,123$ & 65 & CALIFORNIA IRVINE & $9,508,339$ \\
\hline 9 & CHICAGO & $17,704,138$ & 66 & BOSTON & $9,468,057$ \\
\hline 10 & TEXAS & $17,615,691$ & 67 & IOWA STATE & $9,359,327$ \\
\hline 11 & TEXAS A\&M & $17,002,600$ & 68 & LOUISVILLE & $9,206,025$ \\
\hline 12 & ALBERTA & $16,880,131$ & 69 & ILLINOIS CHICAGO & $9,128,496$ \\
\hline 13 & MINNESOTA & $16,578,284$ & 70 & WAYNE STATE & $9,007,142$ \\
\hline 14 & DUKE & $16,507,634$ & 71 & CONNECTICUT & $8,984,063$ \\
\hline 15 & INDIANA & $16,504,866$ & 72 & BOSTON COLLEGE & $8,979,309$ \\
\hline 16 & NEW YORK & $16,498,536$ & 73 & SASKATCHEWAN & $8,942,165$ \\
\hline 17 & NORTH CAROLINA & $16,322,573$ & 74 & HOUSTON & $8,911,877$ \\
\hline 18 & MCGILL & $16,085,628$ & 75 & MIT & $8,886,555$ \\
\hline 19 & CORNELL & $15,843,247$ & 76 & DARTMOUTH & $8,871,179$ \\
\hline 20 & PENNSYLVANIA & $15,168,859$ & 77 & DELAWARE & $8,809,088$ \\
\hline 21 & CALIFORNIA LOS ANGELES & $15,000,546$ & 78 & NEW MEXICO & $8,676,839$ \\
\hline 22 & BRITISH COLUMBIA & $14,917,231$ & 79 & SUNY-BUFFALO & $8,653,954$ \\
\hline 23 & EMORY & $14,902,358$ & 80 & MISSOURI & $8,515,580$ \\
\hline 24 & WASHINGTON & $14,862,427$ & 81 & BROWN & $8,494,260$ \\
\hline 25 & PITTSBURGH & $14,857,024$ & 82 & MANITOBA & $8,330,789$ \\
\hline 26 & JOHNS HOPKINS & $14,824,216$ & 83 & MCMASTER & $8,261,937$ \\
\hline 27 & OKLAHOMA & $14,081,833$ & 84 & TULANE & $8,188,561$ \\
\hline 28 & ILLINOIS URBANA & $14,065,662$ & 85 & FLORIDA STATE & $8,183,466$ \\
\hline 29 & NORTHWESTERN & $13,596,926$ & 86 & CALIFORNIA DAVIS & $8,113,229$ \\
\hline 30 & IOWA & $13,590,872$ & 87 & SOUTH CAROLINA & $8,051,804$ \\
\hline 31 & VIRGINIA & $13,467,652$ & 88 & UTAH & $7,980,542$ \\
\hline 32 & MIAMI & $13,213,588$ & 89 & ROCHESTER & $7,809,582$ \\
\hline 33 & OHIO STATE & $13,178,838$ & 90 & ALABAMA & $7,688,246$ \\
\hline 34 & ARIZONA & $12,627,388$ & 91 & LOUISIANA STATE & $7,386,879$ \\
\hline 35 & WASHINGTON U.-ST. LOUIS & $12,462,732$ & 92 & HAWAII & $7,165,800$ \\
\hline 36 & FLORIDA & $12,427,750$ & 93 & SUNY-STONY BROOK & $7,111,135$ \\
\hline 37 & TENNESSEE & $12,210,020$ & 94 & VIRGINIA TECH & $7,043,044$ \\
\hline 38 & WESTERN ONTARIO & $11,637,880$ & 95 & NEBRASKA & $7,039,988$ \\
\hline 39 & NOTRE DAME & $11,606,437$ & 96 & SYRACUSE & $7,037,903$ \\
\hline 40 & GEORGIA & $11,605,489$ & 97 & SOUTHERN ILLINOIS & $7,031,254$ \\
\hline 41 & TEMPLE & $11,602,950$ & 98 & OKLAHOMA STATE & $6,841,311$ \\
\hline 42 & VANDERBILT & $11,435,792$ & 99 & OREGON & $6,718,280$ \\
\hline 43 & ARIZONA STATE & $11,433,551$ & 100 & CASE WESTERN RESERVE & $6,702,608$ \\
\hline 44 & BRIGHAM YOUNG & $11,307,847$ & 101 & COLORADO STATE & $6,545,964$ \\
\hline 45 & MONTREAL & $11,294,805$ & 102 & MASSACHUSETTS & $6,545,019$ \\
\hline 46 & MICHIGAN STATE & $11,267,304$ & 103 & WATERLOO & $6,533,760$ \\
\hline 47 & GEORGETOWN & $11,242,786$ & 104 & WASHINGTON STATE & $6,424,476$ \\
\hline 48 & SOUTHERN CALIFORNIA & $11,132,454$ & 105 & GEORGIA TECH & $6,205,541$ \\
\hline 49 & RICE & $11,128,043$ & 106 & CALIFORNIA SANTA BARBARA & $5,798,983$ \\
\hline 50 & TEXAS TECH & $11,079,689$ & 107 & CALIFORNIA RIVERSIDE & $5,745,878$ \\
\hline 51 & COLORADO & $11,014,761$ & 108 & GUELPH & $5,684,408$ \\
\hline 52 & WISCONSIN & $10,974,532$ & 109 & $\mathrm{OHIO}$ & $5,641,009$ \\
\hline 53 & YORK & $10,771,539$ & 110 & AUBURN & $5,629,762$ \\
\hline 54 & PURDUE & $10,580,220$ & 111 & SUNY-ALBANY & $5,404,801$ \\
\hline 55 & RUTGERS & $10,454,644$ & 112 & KENT STATE & $4,042,951$ \\
\hline 56 & KENTUCKY & $10,416,077$ & 113 & HOWARD & $3,823,732$ \\
\hline 57 & GEORGE WASHINGTON & $10,388,129$ & & & \\
\hline
\end{tabular}


Rank Order Table 7: Total Salaries \& Wages Expenditures

\begin{tabular}{|c|c|c|c|c|c|}
\hline RANK & INSTITUTION & VALUE & RANK & INSTITUTION & V ALUE \\
\hline 1 & HARVARD & $62,798,140$ & 58 & VANDERBILT & $10,159,649$ \\
\hline 2 & YALE & $35,781,076$ & 59 & GEORGIA & $10,106,243$ \\
\hline 3 & TORONTO & $35,661,466$ & 60 & CALIFORNIA IRVINE & $10,083,397$ \\
\hline 4 & COLUMBIA & $30,654,868$ & 61 & TENNESSEE & $9,903,904$ \\
\hline 5 & CALIFORNIA BERKELEY & $28,822,747$ & 62 & GEORGE WASHINGTON & $9,888,770$ \\
\hline 6 & CALIFORNIA LOS ANGELES & $28,197,468$ & 63 & MIAMI & $9,641,606$ \\
\hline 7 & MICHIGAN & $25,853,159$ & 64 & TEXAS TECH & $9,515,758$ \\
\hline 8 & PENNSYLVANIA STATE & $24,436,622$ & 65 & WAYNE STATE & $9,349,366$ \\
\hline 9 & WISCONSIN & $23,459,430$ & 66 & BOSTON & $9,170,585$ \\
\hline 10 & CORNELL & $22,666,808$ & 67 & BROWN & $9,161,991$ \\
\hline 11 & WASHINGTON & $22,029,970$ & 68 & HAWAII & $9,087,639$ \\
\hline 12 & ILLINOIS URBANA & $21,011,462$ & 69 & ROCHESTER & $9,019,190$ \\
\hline 13 & TEXAS & $20,772,654$ & 70 & BOSTON COLLEGE & $9,001,260$ \\
\hline 14 & NEW YORK & $20,703,344$ & 71 & CALIFORNIA SANTA BARBARA & $8,840,547$ \\
\hline 15 & RUTGERS & $19,524,672$ & 72 & COLORADO & $8,692,805$ \\
\hline 16 & NORTH CAROLINA & $18,943,723$ & 73 & WESTERN ONTARIO & $8,628,993$ \\
\hline 17 & PRINCETON & $18,789,083$ & 74 & SYRACUSE & $8,489,906$ \\
\hline 18 & ALBERTA & $18,485,514$ & 75 & DARTMOUTH & $8,440,615$ \\
\hline 19 & MINNESOTA & $18,117,695$ & 76 & QUEEN'S & $8,417,136$ \\
\hline 20 & MONTREAL & $17,935,683$ & 77 & KENTUCKY & $8,256,893$ \\
\hline 21 & INDIANA & $17,164,613$ & 78 & CINCINNATI & $8,004,337$ \\
\hline 22 & SOUTHERN CALIFORNIA & $17,149,316$ & 79 & SOUTH CAROLINA & $7,974,800$ \\
\hline 23 & PENNSYLVANIA & $16,991,224$ & 80 & TEMPLE & $7,940,588$ \\
\hline 24 & VIRGINIA & $16,921,233$ & 81 & ILLINOIS CHICAGO & $7,873,483$ \\
\hline 25 & OHIO STATE & $16,642,379$ & 82 & OKLAHOMA STATE & $7,868,617$ \\
\hline 26 & DUKE & $16,443,967$ & 83 & DELAWARE & $7,824,331$ \\
\hline 27 & CALIFORNIA SAN DIEGO & $16,330,438$ & 84 & CALIFORNIA RIVERSIDE & $7,823,209$ \\
\hline 28 & BRITISH COLUMBIA & $16,006,535$ & 85 & MCMASTER & $7,739,018$ \\
\hline 29 & CONNECTICUT & $14,751,755$ & 86 & OREGON & $7,678,922$ \\
\hline 30 & MCGILL & $14,495,187$ & 87 & SASKATCHEWAN & $7,637,931$ \\
\hline 31 & FLORIDA & $13,953,783$ & 88 & MASSACHUSETTS & $7,611,671$ \\
\hline 32 & EMORY & $12,967,829$ & 89 & IOWA STATE & $7,283,798$ \\
\hline 33 & UTAH & $12,876,958$ & 90 & WATERLOO & $7,152,770$ \\
\hline 34 & MIT & $12,686,517$ & 91 & FLORIDA STATE & $7,089,568$ \\
\hline 35 & CHICAGO & $12,637,670$ & 92 & NEBRASKA & $7,050,813$ \\
\hline 36 & MARYLAND & $12,599,615$ & 93 & ALABAMA & $6,814,650$ \\
\hline 37 & PITTSBURGH & $12,538,822$ & 94 & WASHINGTON STATE & $6,722,459$ \\
\hline 38 & NORTHWESTERN & $12,517,607$ & 95 & SOUTHERN ILLINOIS & $6,698,054$ \\
\hline 39 & ARIZONA & $12,447,301$ & 96 & OKLAHOMA & $6,650,965$ \\
\hline 40 & IOWA & $12,335,365$ & 97 & SUNY-ALBANY & $6,646,180$ \\
\hline 41 & TEXAS A\&M & $12,328,831$ & 98 & GUELPH & $6,553,835$ \\
\hline 42 & ARIZONA STATE & $12,265,857$ & 99 & SUNY-STONY BROOK & $6,390,259$ \\
\hline 43 & BRIGHAM YOUNG & $12,125,585$ & 100 & MISSOURI & $6,385,653$ \\
\hline 44 & NEW MEXICO & $12,005,115$ & 101 & LOUISVILLE & $6,275,745$ \\
\hline 45 & YORK & $11,932,252$ & 102 & COLORADO STATE & $6,074,168$ \\
\hline 46 & MANITOBA & $11,753,362$ & 103 & LOUISIANA STATE & $5,798,001$ \\
\hline 47 & GEORGETOWN & $11,741,152$ & 104 & HOUSTON & $5,631,937$ \\
\hline 48 & MICHIGAN STATE & $11,669,365$ & 105 & VIRGINIA TECH & $5,615,708$ \\
\hline 49 & PURDUE & $11,043,835$ & 106 & CASE WESTERN RESERVE & $5,565,232$ \\
\hline 50 & NORTH CAROLINA STATE & $10,959,621$ & 107 & $\mathrm{OHIO}$ & $5,477,784$ \\
\hline 51 & JOHNS HOPKINS & $10,523,056$ & 108 & HOWARD & $5,435,311$ \\
\hline 52 & SUNY-BUFFALO & $10,498,307$ & 109 & TULANE & $5,195,043$ \\
\hline 53 & CALIFORNIA DAVIS & $10,443,956$ & 110 & RICE & $5,146,393$ \\
\hline 54 & NOTRE DAME & $10,306,427$ & 111 & GEORGIA TECH & $5,122,524$ \\
\hline 55 & KANSAS & $10,299,504$ & 112 & KENT STATE & $4,804,978$ \\
\hline 56 & WASHINGTON U.-ST. LOUIS & $10,219,063$ & 113 & AUBURN & $4,511,641$ \\
\hline 57 & LAVAL & $10,209,086$ & & & \\
\hline
\end{tabular}


Rank Order Table 8: Other Operating Expenditures

\begin{tabular}{|c|c|c|c|c|c|}
\hline RANK & INSTITUTION & Value & RANK & INSTITUTION & VALUE \\
\hline 1 & HARVARD & $19,683,709$ & 58 & COLORADO STATE & $2,523,449$ \\
\hline 2 & YALE & $11,815,578$ & 59 & GEORGIA & $2,485,368$ \\
\hline 3 & ALBERTA & $11,174,043$ & 60 & MICHIGAN STATE & $2,467,965$ \\
\hline 4 & SOUTHERN CALIFORNIA & $9,953,703$ & 61 & KANSAS & $2,415,971$ \\
\hline 5 & CALIFORNIA LOS ANGELES & $9,537,404$ & 62 & WAYNE STATE & $2,365,219$ \\
\hline 6 & OHIO STATE & $8,329,578$ & 63 & RUTGERS & $2,342,995$ \\
\hline 7 & WISCONSIN & $8,260,282$ & 64 & ILLINOIS CHICAGO & $2,256,901$ \\
\hline 8 & CORNELL & $8,128,897$ & 65 & BOSTON & $2,208,384$ \\
\hline 9 & NORTH CAROLINA STATE & $7,270,144$ & 66 & CONNECTICUT & $2,198,984$ \\
\hline 10 & COLUMBIA & $6,974,601$ & 67 & OKLAHOMA STATE & $2,163,237$ \\
\hline 11 & NEW YORK & $6,930,149$ & 68 & BROWN & $2,126,374$ \\
\hline 12 & CALIFORNIA BERKELEY & $6,674,045$ & 69 & FLORIDA & $2,070,900$ \\
\hline 13 & ROCHESTER & $6,361,120$ & 70 & NOTRE DAME & $2,061,290$ \\
\hline 14 & TORONTO & $6,354,878$ & 71 & ALABAMA & $2,052,276$ \\
\hline 15 & ARIZONA & $6,217,440$ & 72 & OREGON & $1,973,830$ \\
\hline 16 & MINNESOTA & $5,740,315$ & 73 & MARYLAND & $1,937,553$ \\
\hline 17 & NORTH CAROLINA & $5,591,823$ & 74 & OKLAHOMA & $1,912,562$ \\
\hline 18 & PENNSYLVANIA & $5,534,612$ & 75 & DELAWARE & $1,802,698$ \\
\hline 19 & CALIFORNIASANTA BARBARA & $5,435,713$ & 76 & MANITOBA & $1,799,754$ \\
\hline 20 & VIRGINIA & $5,407,540$ & 77 & CINCINNATI & $1,783,625$ \\
\hline 21 & TEXAS & $5,259,179$ & 78 & MASSACHUSETTS & $1,741,283$ \\
\hline 22 & INDIANA & $5,129,811$ & 79 & WATERLOO & $1,738,974$ \\
\hline 23 & PENNSYLVANIA STATE & $5,021,538$ & 80 & IOWA STATE & $1,724,165$ \\
\hline 24 & PRINCETON & $4,919,618$ & 81 & MISSOURI & $1,690,135$ \\
\hline 25 & MICHIGAN & $4,782,459$ & 82 & CALIFORNIA DAVIS & $1,677,155$ \\
\hline 26 & TEXAS A\&M & $4,705,074$ & 83 & SUNY-BUFFALO & $1,567,889$ \\
\hline 27 & HOUSTON & $4,611,134$ & 84 & AUBURN & $1,552,088$ \\
\hline 28 & WASHINGTON U.-ST. LOUIS & $4,556,365$ & 85 & SYRACUSE & $1,549,946$ \\
\hline 29 & TEXAS TECH & $4,497,118$ & 86 & IOWA & $1,542,269$ \\
\hline 30 & ILLINOIS URBANA & $4,351,948$ & 87 & COLORADO & $1,521,928$ \\
\hline 31 & MCGILL & $4,316,278$ & 88 & WASHINGTON STATE & $1,517,246$ \\
\hline 32 & MIAMI & $4,304,519$ & 89 & QUEEN'S & $1,502,065$ \\
\hline 33 & CALIFORNIA SAN DIEGO & $4,282,331$ & 90 & SOUTHERN ILLINOIS & $1,443,490$ \\
\hline 34 & DUKE & $4,181,879$ & 91 & MCMASTER & $1,404,406$ \\
\hline 35 & PITTSBURGH & $4,068,526$ & 92 & NEBRASKA & $1,382,252$ \\
\hline 36 & BRITISH COLUMBIA & $3,955,552$ & 93 & CALIFORNIA RIVERSIDE & $1,378,904$ \\
\hline 37 & EMORY & $3,932,346$ & 94 & LOUISIANA STATE & $1,341,854$ \\
\hline 38 & PURDUE & $3,860,300$ & 95 & TENNESSEE & $1,338,868$ \\
\hline 39 & CHICAGO & $3,841,968$ & 96 & DARTMOUTH & $1,337,837$ \\
\hline 40 & YORK & $3,779,397$ & 97 & HAWAII & $1,293,683$ \\
\hline 41 & WASHINGTON & $3,749,314$ & 98 & BOSTON COLLEGE & $1,250,914$ \\
\hline 42 & JOHNS HOPKINS & $3,726,050$ & 99 & SASKATCHEWAN & $1,248,714$ \\
\hline 43 & UTAH & $3,724,178$ & 100 & OHIO & $1,233,362$ \\
\hline 44 & SOUTH CAROLINA & $3,643,160$ & 101 & MONTREAL & $1,205,676$ \\
\hline 45 & BRIGHAM YOUNG & $3,616,792$ & 102 & GUELPH & $1,157,100$ \\
\hline 46 & LOUISVILLE & $3,564,899$ & 103 & GEORGIA TECH & $1,066,271$ \\
\hline 47 & ARIZONA STATE & $3,505,023$ & 104 & CASE WESTERN RESERVE & $1,064,181$ \\
\hline 48 & NEW MEXICO & $3,319,369$ & 105 & VIRGINIA TECH & $1,010,566$ \\
\hline 49 & MIT & $3,276,441$ & 106 & HOWARD & 951,272 \\
\hline 50 & TEMPLE & $3,109,273$ & 107 & WESTERN ONTARIO & 934,680 \\
\hline 51 & GEORGE WASHINGTON & $3,068,084$ & 108 & SUNY-STONY BROOK & 749,058 \\
\hline 52 & VANDERBILT & $3,004,230$ & 109 & TULANE & 748,643 \\
\hline 53 & KENT STATE & $2,958,811$ & 110 & RICE & 714,789 \\
\hline 54 & NORTHWESTERN & $2,857,993$ & 111 & FLORIDA STATE & 644,076 \\
\hline 55 & GEORGETOWN & $2,820,784$ & 112 & LAVAL & 607,324 \\
\hline 56 & CALIFORNIA IRVINE & $2,702,701$ & 113 & SUNY-ALBANY & 75,884 \\
\hline 57 & KENTUCKY & $2,597,096$ & & & \\
\hline
\end{tabular}


Rank Order Table 9: Total Library Expenditures

\begin{tabular}{|c|c|c|c|c|c|}
\hline RANK & INSTITUTION & VALUE & RANK & INSTITUTION & V ALUE \\
\hline 1 & HARVARD & $117,884,297$ & 58 & GEORGE WASHINGTON & $23,490,425$ \\
\hline 2 & YALE & $81,457,470$ & 59 & ROCHESTER & $23,301,685$ \\
\hline 3 & TORONTO & $68,154,539$ & 60 & OKLAHOMA & $22,863,907$ \\
\hline 4 & COLUMBIA & $61,949,877$ & 61 & TEMPLE & $22,794,168$ \\
\hline 5 & CALIFORNIA BERKELEY & $56,670,387$ & 62 & KANSAS & $22,432,493$ \\
\hline 6 & CALIFORNIA LOS ANGELES & $53,153,870$ & 63 & CALIFORNIA IRVINE & $22,359,838$ \\
\hline 7 & MICHIGAN & $51,599,110$ & 64 & MANITOBA & $22,044,012$ \\
\hline 8 & PENNSYLVANIA STATE & $47,686,386$ & 65 & KENTUCKY & $21,414,484$ \\
\hline 9 & CORNELL & $46,797,533$ & 66 & COLORADO & $21,337,928$ \\
\hline 10 & ALBERTA & $46,622,403$ & 67 & WESTERN ONTARIO & $21,317,718$ \\
\hline 11 & PRINCETON & $45,419,609$ & 68 & LAVAL & $21,267,530$ \\
\hline 12 & NEW YORK & $44,602,730$ & 69 & BOSTON & $21,007,389$ \\
\hline 13 & TEXAS & $43,850,006$ & 70 & WAYNE STATE & $20,801,616$ \\
\hline 14 & WISCONSIN & $42,879,223$ & 71 & SUNY-BUFFALO & $20,787,929$ \\
\hline 15 & NORTH CAROLINA & $41,123,515$ & 72 & CALIFORNIA DAVIS & $20,387,728$ \\
\hline 16 & WASHINGTON & $40,854,830$ & 73 & CALIFORNIA SANTA BARBARA & $20,227,792$ \\
\hline 17 & MINNESOTA & $40,734,045$ & 74 & CINCINNATI & $20,205,911$ \\
\hline 18 & ILLINOIS URBANA & $39,714,492$ & 75 & BROWN & $19,861,584$ \\
\hline 19 & INDIANA & $39,018,150$ & 76 & SOUTH CAROLINA & $19,742,585$ \\
\hline 20 & OHIO STATE & $38,473,238$ & 77 & QUEEN'S & $19,548,008$ \\
\hline 21 & SOUTHERN CALIFORNIA & $38,393,209$ & 78 & BOSTON COLLEGE & $19,328,235$ \\
\hline 22 & PENNSYLVANIA & $38,011,711$ & 79 & HOUSTON & $19,286,301$ \\
\hline 23 & DUKE & $37,330,588$ & 80 & ILLINOIS CHICAGO & $19,258,880$ \\
\hline 24 & VIRGINIA & $35,929,803$ & 81 & LOUISVILLE & $19,154,127$ \\
\hline 25 & MCGILL & $35,143,367$ & 82 & DARTMOUTH & $18,767,039$ \\
\hline 26 & BRITISH COLUMBIA & $35,014,948$ & 83 & IOWA STATE & $18,494,395$ \\
\hline 27 & CHICAGO & $34,585,402$ & 84 & DELAWARE & $18,478,586$ \\
\hline 28 & TEXAS A\&M & $34,149,651$ & 85 & SASKATCHEWAN & $17,901,568$ \\
\hline 29 & RUTGERS & $32,434,060$ & 86 & HAWAII & $17,690,130$ \\
\hline 30 & EMORY & $31,936,414$ & 87 & MCMASTER & $17,409,428$ \\
\hline 31 & PITTSBURGH & $31,660,109$ & 88 & SYRACUSE & $17,128,985$ \\
\hline 32 & ARIZONA & $31,355,788$ & 89 & RICE & $17,039,305$ \\
\hline 33 & MONTREAL & $30,576,387$ & 90 & OKLAHOMA STATE & $16,942,310$ \\
\hline 34 & CALIFORNIA SAN DIEGO & $30,461,462$ & 91 & MISSOURI & $16,694,070$ \\
\hline 35 & JOHNS HOPKINS & $29,150,588$ & 92 & ALABAMA & $16,623,179$ \\
\hline 36 & NORTHWESTERN & $29,147,141$ & 93 & OREGON & $16,477,261$ \\
\hline 37 & FLORIDA & $28,573,302$ & 94 & MASSACHUSETTS & $15,995,789$ \\
\hline 38 & NORTH CAROLINA STATE & $28,249,762$ & 95 & FLORIDA STATE & $15,962,728$ \\
\hline 39 & IOWA & $27,620,248$ & 96 & NEBRASKA & $15,617,134$ \\
\hline 40 & WASHINGTON U.-ST. LOUIS & $27,347,597$ & 97 & WATERLOO & $15,475,270$ \\
\hline 41 & ARIZONA STATE & $27,342,037$ & 98 & SOUTHERN ILLINOIS & $15,297,899$ \\
\hline 42 & MIAMI & $27,250,844$ & 99 & COLORADO STATE & $15,187,932$ \\
\hline 43 & BRIGHAM YOUNG & $27,167,004$ & 100 & CALIFORNIA RIVERSIDE & $15,129,803$ \\
\hline 44 & YORK & $26,661,107$ & 101 & WASHINGTON STATE & $14,709,942$ \\
\hline 45 & CONNECTICUT & $26,099,917$ & 102 & LOUISIANA STATE & $14,576,026$ \\
\hline 46 & GEORGETOWN & $25,913,015$ & 103 & SUNY-STONY BROOK & $14,311,031$ \\
\hline 47 & MICHIGAN STATE & $25,606,985$ & 104 & TULANE & $14,177,339$ \\
\hline 48 & PURDUE & $25,573,918$ & 105 & VIRGINIA TECH & $13,720,067$ \\
\hline 49 & TEXAS TECH & $25,184,730$ & 106 & GUELPH & $13,429,656$ \\
\hline 50 & MIT & $25,046,632$ & 107 & CASE WESTERN RESERVE & $13,401,474$ \\
\hline 51 & MARYLAND & $24,748,579$ & 108 & GEORGIA TECH & $12,432,754$ \\
\hline 52 & VANDERBILT & $24,727,583$ & 109 & OHIO & $12,390,474$ \\
\hline 53 & UTAH & $24,695,844$ & 110 & SUNY-ALBANY & $12,183,367$ \\
\hline 54 & GEORGIA & $24,451,142$ & 111 & KENT STATE & $11,883,778$ \\
\hline 55 & NEW MEXICO & $24,084,535$ & 112 & AUBURN & $11,741,607$ \\
\hline 56 & NOTRE DAME & $24,077,230$ & 113 & HOWARD & $10,273,315$ \\
\hline 57 & TENNESSEE & $23,556,230$ & & & \\
\hline
\end{tabular}


Rank Order Table 10: Monographs Purchased (Volumes)

\begin{tabular}{|c|c|c|c|c|c|}
\hline RANK & INSTITUTION & VAlue & RANK & INSTITUTION & VALUE \\
\hline 1 & COLUMBIA & 468,515 & 58 & OKLAHOMA & 30,327 \\
\hline 2 & MCGILL & 378,866 & 59 & BOSTON COLLEGE & 29,728 \\
\hline 3 & YALE & 167,433 & 60 & DARTMOUTH & 29,420 \\
\hline 4 & BRIGHAM YOUNG & 153,553 & 61 & NORTH CAROLINA STATE & 29,085 \\
\hline 5 & TORONTO & 127,855 & 62 & IOWA STATE & 28,436 \\
\hline 6 & CHICAGO & 125,542 & 63 & CALIFORNIA SANTA BARBARA & 28,273 \\
\hline 7 & PRINCETON & 103,438 & 64 & VANDERBILT & 28,145 \\
\hline 8 & MICHIGAN & 99,784 & 65 & DELAWARE & 27,944 \\
\hline 9 & INDIANA & 85,681 & 66 & OHIO & 27,809 \\
\hline 10 & NORTH CAROLINA & 73,359 & 67 & QUEEN'S & 27,734 \\
\hline 11 & ILLINOIS URBANA & 72,722 & 68 & WESTERN ONTARIO & 27,642 \\
\hline 12 & DUKE & 67,879 & 69 & LOUISVILLE & 27,300 \\
\hline 13 & OHIO STATE & 67,611 & 70 & COLORADO STATE & 26,797 \\
\hline 14 & TEXAS A\&M & 64,803 & 71 & WASHINGTON U.-ST. LOUIS & 26,689 \\
\hline 15 & IOWA & 61,470 & 72 & JOHNS HOPKINS & 26,671 \\
\hline 16 & PENNSYLVANIA STATE & 59,330 & 73 & MISSOURI & 26,196 \\
\hline 17 & WATERLOO & 57,664 & 74 & CALIFORNIA RIVERSIDE & 25,318 \\
\hline 18 & CALIFORNIA LOS ANGELES & 56,557 & 75 & SUNY-BUFFALO & 25,253 \\
\hline 19 & VIRGINIA & 56,260 & 76 & HOUSTON & 25,062 \\
\hline 20 & WISCONSIN & 55,530 & 77 & GEORGE WASHINGTON & 24,738 \\
\hline 21 & NEW YORK & 55,503 & 78 & WAYNE STATE & 23,615 \\
\hline 22 & TEXAS TECH & 53,560 & 79 & MIT & 23,268 \\
\hline 23 & SOUTHERN CALIFORNIA & 53,374 & 80 & OKLAHOMA STATE & 22,598 \\
\hline 24 & MICHIGAN STATE & 52,719 & 81 & OREGON & 22,450 \\
\hline 25 & ARIZONA STATE & 52,392 & 82 & ROCHESTER & 21,476 \\
\hline 26 & KANSAS & 51,625 & 83 & RUTGERS & 21,183 \\
\hline 27 & WASHINGTON & 51,043 & 84 & HAWAII & 21,005 \\
\hline 28 & MONTREAL & 50,515 & 85 & SASKATCHEWAN & 20,877 \\
\hline 29 & EMORY & 50,483 & 86 & SYRACUSE & 20,601 \\
\hline 30 & MANITOBA & 50,404 & 87 & NEW MEXICO & 20,116 \\
\hline 31 & BROWN & 49,939 & 88 & CONNECTICUT & 19,975 \\
\hline 32 & NOTRE DAME & 49,727 & 89 & VIRGINIA TECH & 19,391 \\
\hline 33 & TEXAS & 48,857 & 90 & SOUTH CAROLINA & 18,830 \\
\hline 34 & GEORGIA & 48,717 & 91 & MASSACHUSETTS & 18,771 \\
\hline 35 & TEMPLE & 47,318 & 92 & PURDUE & 18,324 \\
\hline 36 & FLORIDA STATE & 45,911 & 93 & GUELPH & 17,399 \\
\hline 37 & MIAMI & 45,768 & 94 & SOUTHERN ILLINOIS & 17,299 \\
\hline 38 & TULANE & 43,740 & 95 & SUNY-ALBANY & 16,845 \\
\hline 39 & MINNESOTA & 43,115 & 96 & WASHINGTON STATE & 15,383 \\
\hline 40 & CALIFORNIA SAN DIEGO & 42,823 & 97 & BOSTON & 15,001 \\
\hline 41 & KENTUCKY & 42,529 & 98 & KENT STATE & 14,724 \\
\hline 42 & RICE & 42,064 & 99 & AUBURN & 12,224 \\
\hline 43 & GEORGETOWN & 41,525 & 100 & CASE WESTERN RESERVE & 10,339 \\
\hline 44 & FLORIDA & 41,448 & 101 & NEBRASKA & 8,302 \\
\hline 45 & LAVAL & 40,357 & 102 & SUNY-STONY BROOK & 8,061 \\
\hline 46 & ILLINOIS CHICAGO & 39,349 & 103 & GEORGIA TECH & 7,926 \\
\hline 47 & NORTHWESTERN & 38,867 & 104 & HOWARD & 4,496 \\
\hline 48 & ARIZONA & 37,870 & & ALBERTA & $\mathrm{UA} / \mathrm{NA}$ \\
\hline 49 & TENNESSEE & 37,010 & & BRITISH COLUMBIA & $\mathrm{UA} / \mathrm{NA}$ \\
\hline 50 & MARYLAND & 36,951 & & CALIFORNIA BERKELEY & $\mathrm{UA} / \mathrm{NA}$ \\
\hline 51 & CALIFORNIA IRVINE & 35,890 & & CALIFORNIA DAVIS & $\mathrm{UA} / \mathrm{NA}$ \\
\hline 52 & YORK & 34,848 & & CORNELL & $\mathrm{UA} / \mathrm{NA}$ \\
\hline 53 & CINCINNATI & 33,903 & & HARVARD & $\mathrm{UA} / \mathrm{NA}$ \\
\hline 54 & LOUISIANA STATE & 33,655 & & PENNSYLVANIA & $\mathrm{UA} / \mathrm{NA}$ \\
\hline 55 & COLORADO & 33,104 & & PITTSBURGH & $\mathrm{UA} / \mathrm{NA}$ \\
\hline 56 & MCMASTER & 32,843 & & UTAH & $\mathrm{UA} / \mathrm{NA}$ \\
\hline 57 & ALABAMA & 32,646 & & & \\
\hline
\end{tabular}


Rank Order Table 11: Expenditures for Monographs

\begin{tabular}{|c|c|c|c|c|c|}
\hline RANK & INSTITUTION & VAlue & RANK & INSTITUTION & V \\
\hline 1 & HARVARD & $13,455,145$ & 58 & JOHNS HOPKINS & $2,030,703$ \\
\hline 2 & YALE & $11,741,599$ & 59 & WISCONSIN & $2,017,313$ \\
\hline 3 & TORONTO & $8,349,661$ & 60 & LOUISIANA STATE & $1,993,211$ \\
\hline 4 & CALIFORNIA BERKELEY & $8,117,984$ & 61 & MONTREAL & $1,976,784$ \\
\hline 5 & PRINCETON & $7,721,342$ & 62 & BROWN & $1,934,956$ \\
\hline 6 & CORNELL & $6,761,733$ & 63 & UTAH & $1,924,324$ \\
\hline 7 & CALIFORNIA LOS ANGELES & $6,516,528$ & 64 & NORTH CAROLINA STATE & $1,862,181$ \\
\hline 8 & INDIANA & $6,427,271$ & 65 & CALIFORNIA SANTA BARBARA & $1,836,596$ \\
\hline 9 & CHICAGO & $6,144,425$ & 66 & TULANE & $1,770,102$ \\
\hline 10 & COLUMBIA & $6,098,651$ & 67 & HOUSTON & $1,760,006$ \\
\hline 11 & ALBERTA & $6,087,053$ & 68 & VANDERBILT & $1,747,797$ \\
\hline 12 & EMORY & $5,908,477$ & 69 & IOWA STATE & $1,719,386$ \\
\hline 13 & DUKE & $5,413,023$ & 70 & WASHINGTON U.-ST. LOUIS & $1,695,048$ \\
\hline 14 & MCGILL & $5,210,714$ & 71 & DARTMOUTH & $1,681,053$ \\
\hline 15 & MICHIGAN & $5,186,157$ & 72 & OREGON & $1,675,671$ \\
\hline 16 & TEXAS A\&M & $5,180,137$ & 73 & QUEEN'S & $1,630,058$ \\
\hline 17 & NOTRE DAME & $4,979,282$ & 74 & MIT & $1,624,976$ \\
\hline 18 & RICE & $4,210,131$ & 75 & KENTUCKY & $1,624,478$ \\
\hline 19 & NEW YORK & $4,017,073$ & 76 & MARYLAND & $1,588,899$ \\
\hline 20 & PENNSYLVANIA STATE & $3,946,715$ & 77 & PURDUE & $1,447,270$ \\
\hline 21 & PENNSYLVANIA & $3,734,557$ & 78 & GUELPH & $1,418,595$ \\
\hline 22 & NORTHWESTERN & $3,631,003$ & 79 & OKLAHOMA STATE & $1,402,560$ \\
\hline 23 & MINNESOTA & $3,436,528$ & 80 & NEW MEXICO & $1,377,722$ \\
\hline 24 & TEXAS & $3,349,386$ & 81 & HAWAII & $1,375,014$ \\
\hline 25 & OKLAHOMA & $3,313,871$ & 82 & BOSTON COLLEGE & $1,368,393$ \\
\hline 26 & BRITISH COLUMBIA & $3,296,520$ & 83 & SOUTH CAROLINA & $1,357,022$ \\
\hline 27 & OHIO STATE & $3,232,837$ & 84 & RUTGERS & $1,352,783$ \\
\hline 28 & BRIGHAM YOUNG & $3,178,353$ & 85 & FLORIDA & $1,348,788$ \\
\hline 29 & NORTH CAROLINA & $3,158,630$ & 86 & COLORADO STATE & $1,322,568$ \\
\hline 30 & MIAMI & $3,147,172$ & 87 & BOSTON & $1,305,954$ \\
\hline 31 & VIRGINIA & $3,117,556$ & 88 & ILLINOIS CHICAGO & $1,292,036$ \\
\hline 32 & WASHINGTON & $3,085,456$ & 89 & FLORIDA STATE & $1,287,909$ \\
\hline 33 & ILLINOIS URBANA & $2,895,921$ & 90 & CALIFORNIA RIVERSIDE & $1,282,122$ \\
\hline 34 & SOUTHERN CALIFORNIA & $2,874,283$ & 91 & OHIO & $1,277,551$ \\
\hline 35 & ARIZONA & $2,857,568$ & 92 & SUNY-BUFFALO & $1,273,433$ \\
\hline 36 & PITTSBURGH & $2,785,410$ & 93 & WATERLOO & $1,267,256$ \\
\hline 37 & CALIFORNIA IRVINE & $2,739,752$ & 94 & SYRACUSE & $1,258,269$ \\
\hline 38 & TEXAS TECH & $2,707,128$ & 95 & ALABAMA & $1,241,164$ \\
\hline 39 & LAVAL & $2,706,726$ & 96 & WAYNE STATE & $1,181,664$ \\
\hline 40 & CALIFORNIA SAN DIEGO & $2,668,605$ & 97 & WASHINGTON STATE & $1,162,960$ \\
\hline 41 & GEORGE WASHINGTON & $2,634,298$ & 98 & ROCHESTER & $1,161,236$ \\
\hline 42 & YORK & $2,577,912$ & 99 & VIRGINIA TECH & $1,126,563$ \\
\hline 43 & GEORGETOWN & $2,467,328$ & 100 & MASSACHUSETTS & $1,084,359$ \\
\hline 44 & ARIZONA STATE & $2,457,075$ & 101 & MCMASTER & $1,058,438$ \\
\hline 45 & CINCINNATI & $2,451,165$ & 102 & MISSOURI & $1,029,157$ \\
\hline 46 & GEORGIA & $2,405,117$ & 103 & CONNECTICUT & 956,188 \\
\hline 47 & IOWA & $2,352,587$ & 104 & LOUISVILLE & 951,788 \\
\hline 48 & CALIFORNIA DAVIS & $2,297,876$ & 105 & SUNY-ALBANY & 885,746 \\
\hline 49 & WESTERN ONTARIO & $2,266,855$ & 106 & KENT STATE & 810,787 \\
\hline 50 & MICHIGAN STATE & $2,244,634$ & 107 & SOUTHERN ILLINOIS & 736,010 \\
\hline 51 & DELAWARE & $2,217,605$ & 108 & CASE WESTERN RESERVE & 719,638 \\
\hline 52 & TEMPLE & $2,216,053$ & 109 & GEORGIA TECH & 571,817 \\
\hline 53 & COLORADO & $2,171,442$ & 110 & AUBURN & 568,640 \\
\hline 54 & TENNESSEE & $2,132,077$ & 111 & NEBRASKA & 427,296 \\
\hline 55 & SASKATCHEWAN & $2,130,561$ & 112 & SUNY-STONY BROOK & 420,700 \\
\hline 56 & KANSAS & $2,084,991$ & 113 & HOWARD & 246,474 \\
\hline 57 & MANITOBA & $2,037,195$ & & & \\
\hline
\end{tabular}


Rank Order Table 12: Current Serials Purchased (Titles)

\begin{tabular}{|c|c|c|c|c|c|}
\hline RANK & INSTITUTION & VALUE & RANK & INSTITUTION & $\overline{\text { VALUE }}$ \\
\hline 1 & YALE & 103,444 & 58 & NEW YORK & 44,503 \\
\hline 2 & ILLINOIS URBANA & 102,120 & 59 & BROWN & 43,144 \\
\hline 3 & COLUMBIA & 102,053 & 60 & OKLAHOMA STATE & 42,658 \\
\hline 4 & ARIZONA & 85,960 & 61 & CALIFORNIA DAVIS & 42,199 \\
\hline 5 & LOUISIANA STATE & 84,991 & 62 & IOWA & 41,760 \\
\hline 6 & CALIFORNIA BERKELEY & 82,162 & 63 & VIRGINIA & 41,440 \\
\hline 7 & PENNSYLVANIA STATE & 80,912 & 64 & HAWAII & 41,356 \\
\hline 8 & OHIO STATE & 78,906 & 65 & YORK & 39,608 \\
\hline 9 & JOHNS HOPKINS & 76,065 & 66 & MCMASTER & 38,683 \\
\hline 10 & PENNSYLVANIA & 72,347 & 67 & COLORADO & 38,140 \\
\hline 11 & MICHIGAN STATE & 71,888 & 68 & CHICAGO & 38,057 \\
\hline 12 & CALIFORNIA RIVERSIDE & 71,266 & 69 & ILLINOIS CHICAGO & 37,912 \\
\hline 13 & CINCINNATI & 70,504 & 70 & TEXAS & 37,342 \\
\hline 14 & SOUTHERN CALIFORNIA & 70,066 & 71 & SOUTHERN ILLINOIS & 37,341 \\
\hline 15 & WASHINGTON U.-ST. LOUIS & 69,400 & 72 & ALBERTA & 36,793 \\
\hline 16 & MIAMI & 69,164 & 73 & EMORY & 36,651 \\
\hline 17 & GEORGE WASHINGTON & 67,881 & 74 & MIT & 36,259 \\
\hline 18 & ARIZONA STATE & 66,037 & 75 & OREGON & 36,029 \\
\hline 19 & BRITISH COLUMBIA & 64,669 & 76 & NORTH CAROLINA & 35,802 \\
\hline 20 & NOTRE DAME & 63,682 & 77 & NEBRASKA & 34,821 \\
\hline 21 & DARTMOUTH & 62,301 & 78 & WAYNE STATE & 34,808 \\
\hline 22 & MCGILL & 58,997 & 79 & TENNESSEE & 34,720 \\
\hline 23 & KENT STATE & 58,662 & 80 & KENTUCKY & 34,333 \\
\hline 24 & BRIGHAM YOUNG & 58,466 & 81 & CALIFORNIA IRVINE & 34,107 \\
\hline 25 & CASE WESTERN RESERVE & 57,889 & 82 & CALIFORNIA SAN DIEGO & 33,938 \\
\hline 26 & RICE & 56,806 & 83 & VANDERBILT & 33,483 \\
\hline 27 & SUNY-BUFFALO & 56,503 & 84 & CONNECTICUT & 32,801 \\
\hline 28 & GEORGIA & 56,454 & 85 & NORTH CAROLINA STATE & 30,902 \\
\hline 29 & MINNESOTA & 56,325 & 86 & MASSACHUSETTS & 29,160 \\
\hline 30 & NEW MEXICO & 55,474 & 87 & PURDUE & 28,822 \\
\hline 31 & WESTERN ONTARIO & 55,094 & 88 & LAVAL & 28,276 \\
\hline 32 & SUNY-STONY BROOK & 53,900 & 89 & CALIFORNIA SANTA BARBARA & 27,333 \\
\hline 33 & LOUISVILLE & 53,461 & 90 & UTAH & 27,168 \\
\hline 34 & TEXAS A\&M & 52,540 & 91 & $\mathrm{OHIO}$ & 26,522 \\
\hline 35 & OKLAHOMA & 52,522 & 92 & MANITOBA & 26,471 \\
\hline 36 & GEORGETOWN & 52,212 & 93 & SYRACUSE & 25,206 \\
\hline 37 & MONTREAL & 51,760 & 94 & MISSOURI & 24,857 \\
\hline 38 & TEXAS TECH & 51,580 & 95 & MARYLAND & 24,441 \\
\hline 39 & BOSTON & 50,529 & 96 & ROCHESTER & 23,705 \\
\hline 40 & PRINCETON & 50,327 & 97 & BOSTON COLLEGE & 21,947 \\
\hline 41 & PITTSBURGH & 49,916 & 98 & COLORADO STATE & 21,688 \\
\hline 42 & WISCONSIN & 49,626 & 99 & SASKATCHEWAN & 21,485 \\
\hline 43 & TORONTO & 49,550 & 100 & DELAWARE & 20,665 \\
\hline 44 & RUTGERS & 49,507 & 101 & HOWARD & 18,295 \\
\hline 45 & INDIANA & 49,490 & 102 & WATERLOO & 16,332 \\
\hline 46 & KANSAS & 49,212 & 103 & SOUTH CAROLINA & 14,629 \\
\hline 47 & QUEEN'S & 48,829 & 104 & AUBURN & 14,030 \\
\hline 48 & ALABAMA & 48,206 & 105 & GEORGIA TECH & 12,515 \\
\hline 49 & DUKE & 47,819 & 106 & VIRGINIA TECH & 11,917 \\
\hline 50 & SUNY-ALBANY & 47,665 & 107 & GUELPH & 11,486 \\
\hline 51 & FLORIDA & 47,449 & & CALIFORNIA LOS ANGELES & $\mathrm{UA} / \mathrm{NA}$ \\
\hline 52 & IOWA STATE & 47,315 & & CORNELL & $\mathrm{UA} / \mathrm{NA}$ \\
\hline 53 & TULANE & 47,295 & & HARVARD & $\mathrm{UA} / \mathrm{NA}$ \\
\hline 54 & NORTHWESTERN & 47,236 & & MICHIGAN & $\mathrm{UA} / \mathrm{NA}$ \\
\hline 55 & TEMPLE & 46,976 & & WASHINGTON & $\mathrm{UA} / \mathrm{NA}$ \\
\hline 56 & HOUSTON & 46,259 & & WASHINGTON STATE & $\mathrm{UA} / \mathrm{NA}$ \\
\hline 57 & FLORIDA STATE & 46,054 & & & \\
\hline
\end{tabular}


Rank Order Table 13: Expenditures for Current Serials

\begin{tabular}{|c|c|c|c|c|c|}
\hline RANK $_{\text {AN }}$ & INSTITUTION & VALUE & RANK & INSTITUTION & $\overline{\text { VALUE }}$ \\
\hline 1 & COLUMBIA & $14,110,787$ & 58 & PURDUE & $7,022,039$ \\
\hline 2 & MICHIGAN & $13,324,502$ & 59 & CONNECTICUT & $6,958,737$ \\
\hline 3 & PENNSYLVANIA STATE & $12,954,911$ & 60 & MIT & $6,817,728$ \\
\hline 4 & CHICAGO & $11,559,713$ & 61 & NORTH CAROLINA STATE & $6,616,791$ \\
\hline 5 & MINNESOTA & $11,501,644$ & 62 & NEW MEXICO & $6,581,497$ \\
\hline 6 & TORONTO & $11,493,126$ & 63 & NOTRE DAME & $6,573,240$ \\
\hline 7 & WASHINGTON & $11,393,157$ & 64 & CALIFORNIA SAN DIEGO & $6,520,966$ \\
\hline 8 & NEW YORK & $11,165,164$ & 65 & CALIFORNIA IRVINE & $6,474,353$ \\
\hline 9 & TEXAS A\&M & $11,101,887$ & 66 & KENTUCKY & $6,421,219$ \\
\hline 10 & JOHNS HOPKINS & $10,842,794$ & 67 & NEBRASKA & $6,401,031$ \\
\hline 11 & PRINCETON & $10,769,945$ & 68 & SASKATCHEWAN & $6,347,299$ \\
\hline 12 & CALIFORNIA BERKELEY & $10,766,841$ & 69 & ALABAMA & $6,329,417$ \\
\hline 13 & BRITISH COLUMBIA & $10,404,252$ & 70 & HOUSTON & $6,275,219$ \\
\hline 14 & MCGILL & $10,187,365$ & 71 & WISCONSIN & $6,263,640$ \\
\hline 15 & ALBERTA & $10,155,590$ & 72 & ROCHESTER & $6,258,440$ \\
\hline 16 & PENNSYLVANIA & $10,090,093$ & 73 & RICE & $6,139,862$ \\
\hline 17 & FLORIDA & $9,938,022$ & 74 & SOUTH CAROLINA & $6,116,438$ \\
\hline 18 & IOWA & $9,918,930$ & 75 & GEORGE WASHINGTON & $6,103,662$ \\
\hline 19 & DUKE & $9,865,125$ & 76 & TULANE & $6,093,411$ \\
\hline 20 & TEXAS & $9,820,242$ & 77 & MISSOURI & $5,985,414$ \\
\hline 21 & MIAMI & $9,739,138$ & 78 & SOUTHERN ILLINOIS & $5,866,259$ \\
\hline 22 & ILLINOIS URBANA & $9,626,380$ & 79 & KANSAS & $5,860,695$ \\
\hline 23 & VANDERBILT & $9,514,270$ & 80 & MANITOBA & $5,822,024$ \\
\hline 24 & NORTH CAROLINA & $9,443,093$ & 81 & DELAWARE & $5,778,112$ \\
\hline 25 & TENNESSEE & $9,432,867$ & 82 & UTAH & $5,739,547$ \\
\hline 26 & HARVARD & $9,248,116$ & 83 & MCMASTER & $5,714,150$ \\
\hline 27 & PITTSBURGH & $9,243,253$ & 84 & TEMPLE & $5,690,849$ \\
\hline 28 & MONTREAL & $9,211,831$ & 85 & HAWAII & $5,630,515$ \\
\hline 29 & WESTERN ONTARIO & $9,129,335$ & 86 & CASE WESTERN RESERVE & $5,626,072$ \\
\hline 30 & NORTHWESTERN & $8,783,955$ & 87 & BROWN & $5,593,056$ \\
\hline 31 & INDIANA & $8,782,161$ & 88 & YALE & $5,557,697$ \\
\hline 32 & OHIO STATE & $8,765,104$ & 89 & VIRGINIA TECH & $5,423,174$ \\
\hline 33 & OKLAHOMA & $8,582,861$ & 90 & FLORIDA STATE & $5,337,897$ \\
\hline 34 & WASHINGTON U.-ST. LOUIS & $8,486,004$ & 91 & SYRACUSE & $5,316,483$ \\
\hline 35 & MICHIGAN STATE & $8,444,193$ & 92 & OKLAHOMA STATE & $5,286,432$ \\
\hline 36 & VIRGINIA & $8,426,644$ & 93 & LOUISIANA STATE & $5,283,693$ \\
\hline 37 & GEORGIA & $8,415,725$ & 94 & SUNY-STONY BROOK & $5,221,576$ \\
\hline 38 & ARIZONA & $8,366,628$ & 95 & BOSTON COLLEGE & $5,125,794$ \\
\hline 39 & ARIZONA STATE & $8,261,537$ & 96 & WASHINGTON STATE & $5,042,750$ \\
\hline 40 & CALIFORNIA LOS ANGELES & $8,021,917$ & 97 & MASSACHUSETTS & $5,028,032$ \\
\hline 41 & CORNELL & $7,934,603$ & 98 & AUBURN & $5,016,185$ \\
\hline 42 & BRIGHAM YOUNG & $7,909,894$ & 99 & EMORY & $4,877,256$ \\
\hline 43 & YORK & $7,881,161$ & 100 & LOUISVILLE & $4,811,709$ \\
\hline 44 & SOUTHERN CALIFORNIA & $7,878,187$ & 101 & WATERLOO & $4,649,880$ \\
\hline 45 & BOSTON & $7,860,431$ & 102 & COLORADO STATE & $4,611,644$ \\
\hline 46 & GEORGETOWN & $7,843,448$ & 103 & CALIFORNIA DAVIS & $4,597,531$ \\
\hline 47 & RUTGERS & $7,774,275$ & 104 & CALIFORNIA RIVERSIDE & $4,338,335$ \\
\hline 48 & MARYLAND & $7,651,874$ & 105 & ILLINOIS CHICAGO & $4,232,443$ \\
\hline 49 & WAYNE STATE & $7,495,718$ & 106 & GEORGIA TECH & $4,227,563$ \\
\hline 50 & QUEEN'S & $7,367,618$ & 107 & SUNY-ALBANY & $4,215,691$ \\
\hline 51 & LAVAL & $7,322,707$ & 108 & OREGON & $3,976,853$ \\
\hline 52 & TEXAS TECH & $7,318,882$ & 109 & CALIFORNIASANTA BARBARA & $3,962,387$ \\
\hline 53 & SUNY-BUFFALO & $7,172,458$ & 110 & $\mathrm{OHIO}$ & $3,642,738$ \\
\hline 54 & COLORADO & $7,158,550$ & 111 & GUELPH & $3,586,461$ \\
\hline 55 & IOWA STATE & $7,105,893$ & 112 & HOWARD & $3,360,936$ \\
\hline 56 & DARTMOUTH & $7,088,387$ & 113 & KENT STATE & $3,232,164$ \\
\hline 57 & CINCINNATI & $7,075,210$ & & & \\
\hline
\end{tabular}


Rank Order Table 14: Total Items Loaned (ILL/DD)

\begin{tabular}{|c|c|c|c|c|c|}
\hline RANK & INSTITUTION & Value & RANK & INSTITUTION & VALUE \\
\hline 1 & OHIO STATE & 170,711 & 58 & TENNESSEE & 35,585 \\
\hline 2 & MINNESOTA & 165,913 & 59 & DARTMOUTH & 35,464 \\
\hline 3 & ILLINOIS URBANA & 93,584 & 60 & OKLAHOMA & 35,148 \\
\hline 4 & WISCONSIN & 92,683 & 61 & SUNY-BUFFALO & 34,257 \\
\hline 5 & WASHINGTON & 86,879 & 62 & NEBRASKA & 33,959 \\
\hline 6 & OREGON & 80,560 & 63 & DUKE & 33,879 \\
\hline 7 & PENNSYLVANIA & 74,659 & 64 & NEW YORK & 33,089 \\
\hline 8 & COLORADO STATE & 70,733 & 65 & CALIFORNIA DAVIS & 32,098 \\
\hline 9 & PENNSYLVANIA STATE & 66,704 & 66 & GEORGE WASHINGTON & 32,010 \\
\hline 10 & PITTSBURGH & 62,936 & 67 & KENTUCKY & 32,008 \\
\hline 11 & CINCINNATI & 62,455 & 68 & IOWA STATE & 29,651 \\
\hline 12 & IOWA & 62,404 & 69 & NOTRE DAME & 29,584 \\
\hline 13 & MICHIGAN STATE & 62,153 & 70 & BOSTON COLLEGE & 28,636 \\
\hline 14 & ILLINOIS CHICAGO & 61,975 & 71 & TORONTO & 28,195 \\
\hline 15 & MICHIGAN & 59,885 & 72 & CALIFORNIA IRVINE & 27,869 \\
\hline 16 & COLORADO & 59,882 & 73 & ROCHESTER & 27,420 \\
\hline 17 & NORTH CAROLINA & 59,026 & 74 & TEMPLE & 27,341 \\
\hline 18 & TEXAS & 57,999 & 75 & PURDUE & 26,430 \\
\hline 19 & SOUTHERN ILLINOIS & 56,952 & 76 & HOUSTON & 26,008 \\
\hline 20 & CORNELL & 56,865 & 77 & VANDERBILT & 25,849 \\
\hline 21 & WATERLOO & 56,757 & 78 & FLORIDA & 25,472 \\
\hline 22 & WASHINGTON STATE & 55,958 & 79 & SOUTHERN CALIFORNIA & 24,627 \\
\hline 23 & ARIZONA & 55,343 & 80 & CALIFORNIASANTA BARBARA & 24,050 \\
\hline 24 & MISSOURI & 55,210 & 81 & LOUISVILLE & 23,048 \\
\hline 25 & PRINCETON & 54,031 & 82 & BRITISH COLUMBIA & 22,884 \\
\hline 26 & ALBERTA & 53,211 & 83 & MARYLAND & 22,215 \\
\hline 27 & CASE WESTERN RESERVE & 52,249 & 84 & VIRGINIA TECH & 22,192 \\
\hline 28 & ARIZONA STATE & 50,966 & 85 & MONTREAL & 22,041 \\
\hline 29 & CHICAGO & 50,664 & 86 & MCGILL & 21,768 \\
\hline 30 & WASHINGTON U.-ST. LOUIS & 50,554 & 87 & NEW MEXICO & 21,224 \\
\hline 31 & KANSAS & 50,502 & 88 & MANITOBA & 20,917 \\
\hline 32 & INDIANA & 50,325 & 89 & BOSTON & 20,839 \\
\hline 33 & CALIFORNIA LOS ANGELES & 49,510 & 90 & SUNY-STONY BROOK & 20,820 \\
\hline 34 & UTAH & 48,592 & 91 & QUEEN'S & 20,064 \\
\hline 35 & YALE & 48,357 & 92 & SOUTH CAROLINA & 19,696 \\
\hline 36 & BRIGHAM YOUNG & 47,231 & 93 & MIT & 19,287 \\
\hline 37 & COLUMBIA & 46,893 & 94 & CALIFORNIA RIVERSIDE & 18,928 \\
\hline 38 & TEXAS A\&M & 45,798 & 95 & FLORIDA STATE & 18,785 \\
\hline 39 & GUELPH & 45,774 & 96 & LAVAL & 17,842 \\
\hline 40 & MASSACHUSETTS & 44,946 & 97 & DELAWARE & 17,207 \\
\hline 41 & HARVARD & 44,808 & 98 & WESTERN ONTARIO & 16,881 \\
\hline 42 & RUTGERS & 43,575 & 99 & TULANE & 16,272 \\
\hline 43 & CALIFORNIA BERKELEY & 42,769 & 100 & MCMASTER & 15,678 \\
\hline 44 & GEORGIA & 42,723 & 101 & NORTH CAROLINA STATE & 14,979 \\
\hline 45 & WAYNE STATE & 42,491 & 102 & KENT STATE & 14,897 \\
\hline 46 & OHIO & 41,847 & 103 & SYRACUSE & 13,775 \\
\hline 47 & TEXAS TECH & 41,739 & 104 & YORK & 13,665 \\
\hline 48 & MIAMI & 41,524 & 105 & SUNY-ALBANY & 13,133 \\
\hline 49 & BROWN & 41,359 & 106 & RICE & 12,332 \\
\hline 50 & CONNECTICUT & 41,259 & 107 & ALABAMA & 12,114 \\
\hline 51 & VIRGINIA & 39,945 & 108 & AUBURN & 12,091 \\
\hline 52 & CALIFORNIA SAN DIEGO & 37,556 & 109 & GEORGIA TECH & 10,598 \\
\hline 53 & GEORGETOWN & 37,306 & 110 & HOWARD & 8,481 \\
\hline 54 & OKLAHOMA STATE & 37,091 & 111 & LOUISIANA STATE & 7,381 \\
\hline 55 & JOHNS HOPKINS & 37,076 & 112 & HAWAII & 6,246 \\
\hline 56 & EMORY & 36,602 & 113 & SASKATCHEWAN & 5,670 \\
\hline 57 & NORTHWESTERN & 36,383 & & & \\
\hline
\end{tabular}


Rank Order Table 15: Total Items Borrowed (ILL/DD)

\begin{tabular}{|c|c|c|c|c|c|}
\hline RANK & INSTITUTION & VALUE & RANK & INSTITUTION & VALUE \\
\hline 1 & OHIO STATE & 96,771 & 58 & TEXAS & 27,822 \\
\hline 2 & WASHINGTON & 92,703 & 59 & PITTSBURGH & 27,305 \\
\hline 3 & WISCONSIN & 88,726 & 60 & NEW MEXICO & 26,959 \\
\hline 4 & PENNSYLVANIA & 83,836 & 61 & CALIFORNIA DAVIS & 26,504 \\
\hline 5 & ILLINOIS URBANA & 78,457 & 62 & BRIGHAM YOUNG & 25,637 \\
\hline 6 & COLORADO STATE & 66,476 & 63 & MANITOBA & 25,400 \\
\hline 7 & OREGON & 61,746 & 64 & TENNESSEE & 25,179 \\
\hline 8 & ILLINOIS CHICAGO & 56,349 & 65 & SUNY-BUFFALO & 24,824 \\
\hline 9 & COLUMBIA & 55,114 & 66 & GEORGETOWN & 24,366 \\
\hline 10 & GUELPH & 54,470 & 67 & KENTUCKY & 24,349 \\
\hline 11 & GEORGE WASHINGTON & 53,439 & 68 & NOTRE DAME & 24,050 \\
\hline 12 & PENNSYLVANIA STATE & 53,334 & 69 & ROCHESTER & 23,921 \\
\hline 13 & TEXAS A\&M & 51,805 & 70 & DELAWARE & 23,688 \\
\hline 14 & OHIO & 49,452 & 71 & BRITISH COLUMBIA & 22,542 \\
\hline 15 & WASHINGTON U.-ST. LOUIS & 48,729 & 72 & NORTH CAROLINA STATE & 22,509 \\
\hline 16 & MASSACHUSETTS & 48,539 & 73 & CHICAGO & 22,312 \\
\hline 17 & WAYNE STATE & 48,058 & 74 & BOSTON COLLEGE & 22,253 \\
\hline 18 & WATERLOO & 47,160 & 75 & VIRGINIA TECH & 21,936 \\
\hline 19 & INDIANA & 44,360 & 76 & CALIFORNIA BERKELEY & 21,922 \\
\hline 20 & PURDUE & 43,940 & 77 & SYRACUSE & 21,580 \\
\hline 21 & MICHIGAN & 42,978 & 78 & TEMPLE & 21,460 \\
\hline 22 & CONNECTICUT & 42,188 & 79 & OKLAHOMA STATE & 21,358 \\
\hline 23 & CINCINNATI & 42,020 & 80 & BOSTON & 21,266 \\
\hline 24 & MINNESOTA & 41,800 & 81 & EMORY & 21,068 \\
\hline 25 & YALE & 41,321 & 82 & SOUTHERN CALIFORNIA & 20,600 \\
\hline 26 & MISSOURI & 41,231 & 83 & SUNY-ALBANY & 20,487 \\
\hline 27 & MARYLAND & 40,713 & 84 & VANDERBILT & 20,273 \\
\hline 28 & BROWN & 40,355 & 85 & LOUISVILLE & 20,210 \\
\hline 29 & SOUTHERN ILLINOIS & 40,037 & 86 & MONTREAL & 19,917 \\
\hline 30 & KANSAS & 39,672 & 87 & SOUTH CAROLINA & 18,538 \\
\hline 31 & JOHNS HOPKINS & 39,481 & 88 & HOUSTON & 18,395 \\
\hline 32 & ARIZONA STATE & 38,561 & 89 & MIT & 17,464 \\
\hline 33 & CALIFORNIA SANTA BARBARA & 37,282 & 90 & IOWA STATE & 17,027 \\
\hline 34 & PRINCETON & 37,211 & 91 & LOUISIANA STATE & 16,916 \\
\hline 35 & ARIZONA & 36,714 & 92 & MCGILL & 16,593 \\
\hline 36 & NEW YORK & 36,634 & 93 & FLORIDA STATE & 14,990 \\
\hline 37 & RUTGERS & 35,952 & 94 & NORTH CAROLINA & 14,975 \\
\hline 38 & CASE WESTERN RESERVE & 35,150 & 95 & CALIFORNIA RIVERSIDE & 14,186 \\
\hline 39 & CALIFORNIA LOS ANGELES & 34,596 & 96 & MIAMI & 13,738 \\
\hline 40 & CORNELL & 33,528 & 97 & SUNY-STONY BROOK & 13,198 \\
\hline 41 & UTAH & 33,349 & 98 & HARVARD & 12,877 \\
\hline 42 & TEXAS TECH & 33,051 & 99 & MCMASTER & 12,726 \\
\hline 43 & OKLAHOMA & 32,030 & 100 & GEORGIA TECH & 12,062 \\
\hline 44 & HAWAII & 31,778 & 101 & TULANE & 10,284 \\
\hline 45 & CALIFORNIA IRVINE & 31,034 & 102 & RICE & 9,908 \\
\hline 46 & WASHINGTON STATE & 30,936 & 103 & ALABAMA & 9,799 \\
\hline 47 & MICHIGAN STATE & 30,895 & 104 & QUEEN'S & 9,722 \\
\hline 48 & VIRGINIA & 30,606 & 105 & LAVAL & 9,720 \\
\hline 49 & DARTMOUTH & 30,481 & 106 & KENT STATE & 9,333 \\
\hline 50 & ALBERTA & 30,463 & 107 & AUBURN & 8,651 \\
\hline 51 & DUKE & 30,179 & 108 & TORONTO & 8,409 \\
\hline 52 & COLORADO & 30,159 & 109 & WESTERN ONTARIO & 8,087 \\
\hline 53 & FLORIDA & 28,823 & 110 & GEORGIA & 6,862 \\
\hline 54 & NEBRASKA & 28,757 & 111 & HOWARD & 5,560 \\
\hline 55 & IOWA & 28,607 & 112 & YORK & 5,233 \\
\hline 56 & CALIFORNIA SAN DIEGO & 28,588 & 113 & SASKATCHEWAN & 4,771 \\
\hline 57 & NORTHWESTERN & 28,342 & & & \\
\hline
\end{tabular}


Rank Order Table 16: Professional Staff (FTE)

\begin{tabular}{|c|c|c|c|c|c|}
\hline RANK & INSTITUTION NAME & Value & RANK & INSTITUTION NAME & VaLUe \\
\hline 1 & HARVARD & 555 & 57 & PURDUE & 84 \\
\hline 2 & COLUMBIA & 328 & 59 & MCGILL & 83 \\
\hline 3 & YALE & 272 & 60 & MIAMI & 82 \\
\hline 4 & CALIFORNIA BERKELEY & 244 & 60 & MICHIGAN STATE & 82 \\
\hline 5 & WISCONSIN & 229 & 62 & GEORGIA & 81 \\
\hline 6 & ILLINOIS URBANA & 199 & 63 & BOSTON COLLEGE & 79 \\
\hline 7 & WASHINGTON & 193 & 64 & CINCINNATI & 76 \\
\hline 8 & DUKE & 185 & 65 & SOUTH CAROLINA & 74 \\
\hline 9 & TORONTO & 184 & 66 & BROWN & 73 \\
\hline 10 & INDIANA & 173 & 66 & CALIFORNIA IRVINE & 73 \\
\hline 11 & CALIFORNIA LOS ANGELES & 169 & 66 & TEMPLE & 73 \\
\hline 11 & MICHIGAN & 169 & 69 & ILLINOIS CHICAGO & 71 \\
\hline 13 & PENNSYLVANIA STATE & 162 & 69 & WESTERN ONTARIO & 71 \\
\hline 14 & NORTH CAROLINA & 148 & 71 & ALABAMA & 70 \\
\hline 15 & TEXAS A\&M & 145 & 71 & NEW MEXICO & 70 \\
\hline 16 & OHIO STATE & 143 & 71 & NOTRE DAME & 70 \\
\hline 16 & PRINCETON & 143 & 71 & OREGON & 70 \\
\hline 18 & TEXAS & 141 & 75 & CHICAGO & 68 \\
\hline 19 & NEW YORK & 139 & 75 & FLORIDA STATE & 68 \\
\hline 20 & PENNSYLVANIA & 137 & 77 & OKLAHOMA STATE & 66 \\
\hline 21 & JOHNS HOPKINS & 136 & 78 & MANITOBA & 64 \\
\hline 22 & SOUTHERN CALIFORNIA & 129 & 79 & SYRACUSE & 63 \\
\hline 23 & NORTH CAROLINA STATE & 124 & 80 & HOUSTON & 62 \\
\hline 24 & BRIGHAM YOUNG & 120 & 80 & YORK & 62 \\
\hline 24 & CORNELL & 120 & 82 & SUNY-ALBANY & 61 \\
\hline 24 & NORTHWESTERN & 120 & 83 & DARTMOUTH & 60 \\
\hline 24 & PITTSBURGH & 120 & 83 & LAVAL & 60 \\
\hline 28 & MARYLAND & 119 & 83 & OKLAHOMA & 60 \\
\hline 29 & BRITISH COLUMBIA & 114 & 86 & CALIFORNIA DAVIS & 59 \\
\hline 30 & MINNESOTA & 111 & 87 & CASE WESTERN RESERVE & 58 \\
\hline 31 & MONTREAL & 110 & 87 & COLORADO & 58 \\
\hline 32 & SUNY-BUFFALO & 106 & 87 & DELAWARE & 58 \\
\hline 32 & VANDERBILT & 106 & 87 & MISSOURI & 58 \\
\hline 34 & TEXAS TECH & 105 & 91 & RICE & 57 \\
\hline 35 & CALIFORNIA SAN DIEGO & 104 & 91 & WASHINGTON STATE & 57 \\
\hline 36 & KANSAS & 103 & 93 & KENT STATE & 56 \\
\hline 36 & MIT & 103 & 93 & MASSACHUSETTS & 56 \\
\hline 38 & BOSTON & 102 & 95 & GUELPH & 54 \\
\hline 38 & FLORIDA & 102 & 95 & NEBRASKA & 54 \\
\hline 40 & VIRGINIA & 101 & 95 & SOUTHERN ILLINOIS & 54 \\
\hline 41 & RUTGERS & 100 & 98 & LOUISVILLE & 53 \\
\hline 42 & IOWA & 98 & 99 & IOWA STATE & 52 \\
\hline 43 & WAYNE STATE & 97 & 99 & LOUISIANA STATE & 52 \\
\hline 44 & GEORGETOWN & 95 & 99 & SASKATCHEWAN & 52 \\
\hline 44 & ROCHESTER & 95 & 102 & COLORADO STATE & 51 \\
\hline 46 & ARIZONA & 94 & 103 & HOWARD & 50 \\
\hline 46 & EMORY & 94 & 104 & CALIFORNIA RIVERSIDE & 49 \\
\hline 48 & ARIZONA STATE & 93 & 105 & CALIFORNIA SANTA BARBARA & 47 \\
\hline 48 & WASHINGTON U.-ST. LOUIS & 93 & 106 & AUBURN & 46 \\
\hline 50 & HAWAII & 92 & 106 & $\mathrm{OHIO}$ & 46 \\
\hline 51 & KENTUCKY & 91 & 108 & GEORGIA TECH & 45 \\
\hline 52 & SUNY-STONY BROOK & 90 & 108 & MCMASTER & 45 \\
\hline 53 & CONNECTICUT & 86 & 108 & QUEEN'S & 45 \\
\hline 53 & TENNESSEE & 86 & 108 & TULANE & 45 \\
\hline 53 & UTAH & 86 & 112 & VIRGINIA TECH & 38 \\
\hline 56 & ALBERTA & 85 & 113 & WATERLOO & 36 \\
\hline 57 & GEORGE WASHINGTON & 84 & & & \\
\hline
\end{tabular}


Rank Order Table 17: Support Staff (FTE)

\begin{tabular}{|c|c|c|c|c|c|}
\hline RANK & INSTITUTION & Value & RANK & INSTITUTION & VALUE \\
\hline 1 & HARVARD & 541 & 58 & WASHINGTON U.-ST. LOUIS & 115 \\
\hline 2 & YALE & 396 & 59 & YORK & 112 \\
\hline 3 & PENNSYLVANIA STATE & 375 & 60 & WESTERN ONTARIO & 111 \\
\hline 4 & TORONTO & 350 & 61 & PURDUE & 110 \\
\hline 5 & CORNELL & 308 & 61 & FLORIDA STATE & 110 \\
\hline 6 & MICHIGAN & 305 & 61 & MISSOURI & 110 \\
\hline 7 & TEXAS & 285 & 64 & COLORADO & 109 \\
\hline 8 & CALIFORNIA LOS ANGELES & 281 & 65 & KANSAS & 108 \\
\hline 9 & MONTREAL & 258 & 66 & NEBRASKA & 106 \\
\hline 10 & COLUMBIA & 246 & 66 & QUEEN'S & 106 \\
\hline 11 & RUTGERS & 238 & 68 & NORTH CAROLINA STATE & 105 \\
\hline 12 & NEW YORK & 227 & 68 & VANDERBILT & 105 \\
\hline 13 & PRINCETON & 225 & 68 & SOUTH CAROLINA & 105 \\
\hline 14 & ALBERTA & 213 & 68 & DELAWARE & 105 \\
\hline 15 & VIRGINIA & 210 & 72 & ILLINOIS CHICAGO & 104 \\
\hline 16 & NORTH CAROLINA & 209 & 73 & BROWN & 101 \\
\hline 17 & WASHINGTON & 206 & 73 & LOUISIANA STATE & 101 \\
\hline 18 & MINNESOTA & 200 & 75 & WATERLOO & 98 \\
\hline 19 & CALIFORNIA BERKELEY & 198 & 76 & BOSTON & 97 \\
\hline 20 & ARIZONA STATE & 195 & 76 & OKLAHOMA & 97 \\
\hline 21 & ILLINOIS URBANA & 194 & 78 & MARYLAND & 95 \\
\hline 22 & CALIFORNIA SAN DIEGO & 193 & 78 & LOUISVILLE & 95 \\
\hline 23 & CHICAGO & 192 & 78 & MCMASTER & 95 \\
\hline 24 & FLORIDA & 190 & 81 & MIT & 94 \\
\hline 25 & UTAH & 187 & 82 & SOUTHERN ILLINOIS & 92 \\
\hline 26 & BRITISH COLUMBIA & 186 & 83 & TEMPLE & 91 \\
\hline 27 & GEORGIA & 184 & 83 & HOUSTON & 91 \\
\hline 28 & INDIANA & 181 & 85 & VIRGINIA TECH & 90 \\
\hline 29 & PENNSYLVANIA & 179 & 86 & IOWA STATE & 88 \\
\hline 30 & PITTSBURGH & 173 & 86 & SASKATCHEWAN & 88 \\
\hline 31 & MCGILL & 172 & 88 & OREGON & 87 \\
\hline 32 & WISCONSIN & 166 & 89 & WAYNE STATE & 86 \\
\hline 33 & OHIO STATE & 156 & 90 & WASHINGTON STATE & 84 \\
\hline 34 & NOTRE DAME & 154 & 91 & ALABAMA & 83 \\
\hline 35 & EMORY & 153 & 92 & CALIFORNIA RIVERSIDE & 79 \\
\hline 35 & LAVAL & 153 & 93 & HAWAII & 77 \\
\hline 37 & ARIZONA & 146 & 94 & OKLAHOMA STATE & 76 \\
\hline 38 & MANITOBA & 145 & 94 & TULANE & 76 \\
\hline 39 & CALIFORNIA DAVIS & 144 & 96 & BOSTON COLLEGE & 73 \\
\hline 40 & DUKE & 143 & 96 & MASSACHUSETTS & 73 \\
\hline 40 & NEW MEXICO & 143 & 98 & CONNECTICUT & 72 \\
\hline 42 & NORTHWESTERN & 140 & 99 & $\mathrm{OHIO}$ & 70 \\
\hline 43 & JOHNS HOPKINS & 139 & 99 & GEORGIA TECH & 70 \\
\hline 44 & KENTUCKY & 136 & 101 & GUELPH & 69 \\
\hline 45 & CALIFORNIA SANTA BARBARA & 135 & 102 & HOWARD & 67 \\
\hline 46 & SOUTHERN CALIFORNIA & 133 & 103 & SUNY-BUFFALO & 66 \\
\hline 46 & TENNESSEE & 133 & 104 & ROCHESTER & 61 \\
\hline 46 & MIAMI & 133 & 104 & SUNY-ALBANY & 61 \\
\hline 49 & TEXAS A\&M & 132 & 106 & BRIGHAM YOUNG & 59 \\
\hline 50 & TEXAS TECH & 127 & 107 & CINCINNATI & 58 \\
\hline 50 & CALIFORNIA IRVINE & 127 & 108 & COLORADO STATE & 54 \\
\hline 52 & IOWA & 125 & 109 & RICE & 53 \\
\hline 53 & GEORGE WASHINGTON & 124 & 110 & CASE WESTERN RESERVE & 45 \\
\hline 54 & MICHIGAN STATE & 122 & 111 & AUBURN & 40 \\
\hline 55 & SYRACUSE & 121 & 112 & KENT STATE & 36 \\
\hline 56 & DARTMOUTH & 117 & 113 & SUNY-STONY BROOK & 26 \\
\hline 57 & GEORGETOWN & 116 & & & \\
\hline
\end{tabular}


Rank Order Table 18: Total Staff (FTE)

\begin{tabular}{|c|c|c|c|c|c|}
\hline RANK & INSTITUTION & Value & RANK & INSTITUTION & VaLue \\
\hline 1 & HARVARD & 1,229 & 57 & MARYLAND & 258 \\
\hline 2 & YALE & 735 & 59 & PURDUE & 255 \\
\hline 3 & COLUMBIA & 701 & 60 & SUNY-BUFFALO & 250 \\
\hline 4 & TORONTO & 673 & 61 & CALIFORNIA DAVIS & 249 \\
\hline 5 & PENNSYLVANIA STATE & 608 & 62 & CALIFORNIA IRVINE & 248 \\
\hline 6 & CALIFORNIA LOS ANGELES & 597 & 62 & FLORIDA STATE & 248 \\
\hline 7 & CALIFORNIA BERKELEY & 582 & 64 & WAYNE STATE & 247 \\
\hline 8 & MICHIGAN & 570 & 65 & VANDERBILT & 241 \\
\hline 9 & WISCONSIN & 553 & 66 & HAWAII & 236 \\
\hline 10 & CORNELL & 549 & 67 & MANITOBA & 233 \\
\hline 11 & TEXAS & 528 & 68 & OKLAHOMA & 232 \\
\hline 12 & WASHINGTON & 523 & 69 & YORK & 231 \\
\hline 13 & ILLINOIS URBANA & 512 & 70 & CALIFORNIASANTA BARBARA & 229 \\
\hline 14 & INDIANA & 470 & 71 & OREGON & 217 \\
\hline 14 & OHIO STATE & 470 & 72 & COLORADO & 216 \\
\hline 16 & NEW YORK & 458 & 72 & SYRACUSE & 216 \\
\hline 17 & NORTH CAROLINA & 452 & 74 & LAVAL & 214 \\
\hline 18 & RUTGERS & 419 & 74 & MIT & 214 \\
\hline 19 & PRINCETON & 410 & 76 & OKLAHOMA STATE & 213 \\
\hline 20 & PENNSYLVANIA & 402 & 76 & ROCHESTER & 213 \\
\hline 21 & MINNESOTA & 394 & 78 & TEMPLE & 209 \\
\hline 22 & BRIGHAM YOUNG & 383 & 79 & BROWN & 208 \\
\hline 23 & PITTSBURGH & 382 & 80 & CONNECTICUT & 207 \\
\hline 24 & FLORIDA & 381 & 81 & DARTMOUTH & 205 \\
\hline 25 & VIRGINIA & 379 & 82 & NEBRASKA & 204 \\
\hline 26 & SOUTHERN CALIFORNIA & 374 & 83 & ILLINOIS CHICAGO & 203 \\
\hline 27 & UTAH & 370 & 84 & ALABAMA & 202 \\
\hline 28 & MONTREAL & 368 & 84 & BOSTON COLLEGE & 202 \\
\hline 29 & CALIFORNIA SAN DIEGO & 367 & 84 & SOUTHERN ILLINOIS & 202 \\
\hline 30 & DUKE & 363 & 87 & HOUSTON & 199 \\
\hline 31 & TEXAS A\&M & 359 & 88 & DELAWARE & 198 \\
\hline 32 & NORTHWESTERN & 344 & 88 & MISSOURI & 198 \\
\hline 33 & BRITISH COLUMBIA & 338 & 90 & WESTERN ONTARIO & 195 \\
\hline 34 & ALBERTA & 334 & 91 & LOUISIANA STATE & 192 \\
\hline 35 & ARIZONA STATE & 332 & 92 & MASSACHUSETTS & 190 \\
\hline 35 & TEXAS TECH & 332 & 93 & LOUISVILLE & 188 \\
\hline 37 & CHICAGO & 323 & 94 & CINCINNATI & 182 \\
\hline 38 & JOHNS HOPKINS & 317 & 95 & HOWARD & 176 \\
\hline 39 & ARIZONA & 314 & 95 & WASHINGTON STATE & 176 \\
\hline 40 & GEORGIA & 304 & 97 & IOWA STATE & 174 \\
\hline 41 & EMORY & 296 & 98 & WATERLOO & 172 \\
\hline 42 & MCGILL & 294 & 99 & QUEEN'S & 169 \\
\hline 43 & MICHIGAN STATE & 288 & 100 & MCMASTER & 163 \\
\hline 44 & GEORGE WASHINGTON & 286 & 101 & CALIFORNIA RIVERSIDE & 159 \\
\hline 44 & KENTUCKY & 286 & 101 & VIRGINIA TECH & 159 \\
\hline 46 & BOSTON & 285 & 103 & OHIO & 153 \\
\hline 47 & KENT STATE & 284 & 103 & SUNY-ALBANY & 153 \\
\hline 48 & KANSAS & 282 & 105 & SASKATCHEWAN & 151 \\
\hline 49 & IOWA & 281 & 106 & SUNY-STONY BROOK & 147 \\
\hline 50 & SOUTH CAROLINA & 278 & 106 & TULANE & 147 \\
\hline 51 & MIAMI & 277 & 108 & GUELPH & 144 \\
\hline 52 & NORTH CAROLINA STATE & 268 & 109 & CASE WESTERN RESERVE & 131 \\
\hline 52 & TENNESSEE & 268 & 110 & COLORADO STATE & 130 \\
\hline 54 & NEW MEXICO & 267 & 111 & GEORGIA TECH & 123 \\
\hline 55 & WASHINGTON U.-ST. LOUIS & 266 & 112 & RICE & 122 \\
\hline 56 & NOTRE DAME & 260 & 113 & AUBURN & 117 \\
\hline 57 & GEORGETOWN & 258 & & & \\
\hline
\end{tabular}


Rank Order Table 19: Expenditures for Electronic Resources

\begin{tabular}{|c|c|c|c|c|c|}
\hline RANK & INSTITUTION & VALUE & RANK & INSTITUTION & $\overline{\text { VAlue }}$ \\
\hline 1 & ALBERTA & $14,521,604$ & 58 & MIT & $5,315,163$ \\
\hline 2 & COLUMBIA & $10,730,848$ & 59 & NEW MEXICO & $5,313,948$ \\
\hline 3 & PENNSYLVANIA STATE & $10,324,719$ & 60 & FLORIDA STATE & $5,306,200$ \\
\hline 4 & PITTSBURGH & $9,577,575$ & 61 & MCMASTER & $5,302,647$ \\
\hline 5 & TORONTO & $9,283,217$ & 62 & CALIFORNIA SAN DIEGO & $5,283,923$ \\
\hline 6 & NEW YORK & $9,226,060$ & 63 & DARTMOUTH & $5,244,385$ \\
\hline 7 & TEXAS A\&M & $9,111,074$ & 64 & BRIGHAM YOUNG & $5,173,184$ \\
\hline 8 & MCGILL & $8,887,022$ & 65 & NORTH CAROLINA & $5,149,059$ \\
\hline 9 & TEXAS & $8,452,972$ & 66 & RUTGERS & $5,007,836$ \\
\hline 10 & PRINCETON & $8,393,567$ & 67 & KANSAS & $4,931,383$ \\
\hline 11 & HARVARD & $8,300,095$ & 68 & TEXAS TECH & $4,928,468$ \\
\hline 12 & MINNESOTA & $8,074,528$ & 69 & SOUTH CAROLINA & $4,912,821$ \\
\hline 13 & DUKE & $8,001,083$ & 70 & CALIFORNIA LOS ANGELES & $4,862,032$ \\
\hline 14 & WAYNE STATE & $7,971,488$ & 71 & JOHNS HOPKINS & $4,843,098$ \\
\hline 15 & MICHIGAN & $7,734,012$ & 72 & BOSTON COLLEGE & $4,808,867$ \\
\hline 16 & BRITISH COLUMBIA & $7,648,588$ & 73 & RICE & $4,757,600$ \\
\hline 17 & VANDERBILT & $7,616,980$ & 74 & CALIFORNIA IRVINE & $4,724,596$ \\
\hline 18 & WASHINGTON & $7,513,531$ & 75 & AUBURN & $4,704,780$ \\
\hline 19 & YALE & $7,355,032$ & 76 & HOUSTON & $4,657,866$ \\
\hline 20 & ARIZONA & $7,346,049$ & 77 & ILLINOIS CHICAGO & $4,646,070$ \\
\hline 21 & NORTHWESTERN & $7,197,977$ & 78 & OKLAHOMA & $4,579,054$ \\
\hline 22 & CORNELL & $7,041,326$ & 79 & BROWN & $4,543,261$ \\
\hline 23 & WESTERN ONTARIO & $6,978,173$ & 80 & EMORY & $4,513,037$ \\
\hline 24 & MIAMI & $6,944,791$ & 81 & TULANE & $4,459,650$ \\
\hline 25 & PENNSYLVANIA & $6,792,252$ & 82 & VIRGINIA TECH & $4,441,724$ \\
\hline 26 & WASHINGTON U.-ST. LOUIS & $6,779,568$ & 83 & MASSACHUSETTS & $4,389,499$ \\
\hline 27 & MONTREAL & $6,666,762$ & 84 & MANITOBA & $4,388,649$ \\
\hline 28 & COLORADO & $6,628,496$ & 85 & CALIFORNIA DAVIS & $4,387,167$ \\
\hline 29 & TEMPLE & $6,601,957$ & 86 & LOUISIANA STATE & $4,272,575$ \\
\hline 30 & OHIO STATE & $6,590,172$ & 87 & COLORADO STATE & $4,192,556$ \\
\hline 31 & INDIANA & $6,587,019$ & 88 & GEORGETOWN & $4,173,607$ \\
\hline 32 & CALIFORNIA BERKELEY & $6,522,404$ & 89 & GEORGIA TECH & $4,158,648$ \\
\hline 33 & NOTRE DAME & $6,491,200$ & 90 & WASHINGTON STATE & $4,145,155$ \\
\hline 34 & PURDUE & $6,393,070$ & 91 & ALABAMA & $4,037,173$ \\
\hline 35 & MARYLAND & $6,384,820$ & 92 & SUNY-STONY BROOK & $3,943,945$ \\
\hline 36 & IOWA STATE & $6,356,897$ & 93 & SOUTHERN ILLINOIS & $3,928,326$ \\
\hline 37 & MICHIGAN STATE & $6,323,616$ & 94 & NORTH CAROLINA STATE & $3,830,451$ \\
\hline 38 & GEORGIA & $6,315,224$ & 95 & SYRACUSE & $3,789,456$ \\
\hline 39 & IOWA & $6,293,902$ & 96 & WATERLOO & $3,722,260$ \\
\hline 40 & MISSOURI & $6,254,447$ & 97 & GUELPH & $3,664,146$ \\
\hline 41 & KENTUCKY & $6,138,460$ & 98 & ROCHESTER & $3,531,633$ \\
\hline 42 & YORK & $6,115,127$ & 99 & CASE WESTERN RESERVE & $3,517,639$ \\
\hline 43 & ARIZONA STATE & $6,066,629$ & 100 & HAWAII & $3,498,685$ \\
\hline 44 & SOUTHERN CALIFORNIA & $5,939,554$ & 101 & BOSTON & $3,362,031$ \\
\hline 45 & ILLINOIS URBANA & $5,921,211$ & 102 & LOUISVILLE & $3,211,731$ \\
\hline 46 & CHICAGO & $5,767,694$ & 103 & $\mathrm{OHIO}$ & $2,872,036$ \\
\hline 47 & VIRGINIA & $5,761,823$ & 104 & OREGON & $2,839,843$ \\
\hline 48 & FLORIDA & $5,681,228$ & 105 & CALIFORNIA SANTA BARBARA & $2,696,936$ \\
\hline 49 & QUEENS & $5,671,832$ & 106 & NEBRASKA & $2,424,217$ \\
\hline 50 & LAVAL & $5,668,355$ & 107 & GEORGE WASHINGTON & $2,391,699$ \\
\hline 51 & TENNESSEE & $5,643,552$ & 108 & CALIFORNIA RIVERSIDE & $2,278,784$ \\
\hline 52 & CINCINNATI & $5,601,049$ & 109 & UTAH & $2,077,201$ \\
\hline 53 & DELAWARE & $5,549,645$ & 110 & HOWARD & $2,075,989$ \\
\hline 54 & SASKATCHEWAN & $5,541,661$ & 111 & SUNY-ALBANY & $1,960,241$ \\
\hline 55 & SUNY-BUFFALO & $5,477,759$ & & KENT STATE & $\mathrm{UA} / \mathrm{NA}$ \\
\hline 56 & CONNECTICUT & $5,410,421$ & & OKLAHOMA STATE & $\mathrm{UA} / \mathrm{NA}$ \\
\hline 57 & WISCONSIN & $5,364,822$ & & & \\
\hline
\end{tabular}


Rank Order Table 20: Electronic Resources as a Percentage of Total Library Materials

\begin{tabular}{|c|c|c|c|c|c|}
\hline$R_{\text {ANK }}$ & INSTITUTION & Value & RANK & INSTITUTION & VALUe \\
\hline 1 & WAYNE STATE & 88.50 & 58 & MANITOBA & 52.68 \\
\hline 2 & ALBERTA & 86.03 & 59 & MIAMI & 52.56 \\
\hline 3 & AUBURN & 83.57 & 60 & ALABAMA & 52.51 \\
\hline 4 & MISSOURI & 73.45 & 61 & CASE WESTERN RESERVE & 52.48 \\
\hline 5 & IOWA STATE & 67.92 & 62 & HOUSTON & 52.27 \\
\hline 6 & MASSACHUSETTS & 67.07 & 63 & KANSAS & 51.47 \\
\hline 7 & GEORGIA TECH & 67.02 & 64 & BRITISH COLUMBIA & 51.27 \\
\hline 8 & VANDERBILT & 66.61 & 65 & $\mathrm{OHIO}$ & 50.91 \\
\hline 9 & FLORIDA STATE & 64.84 & 66 & ILLINOIS CHICAGO & 50.90 \\
\hline 10 & WASHINGTON STATE & 64.52 & 67 & WASHINGTON & 50.55 \\
\hline 11 & PITTSBURGH & 64.46 & 68 & OHIO STATE & 50.01 \\
\hline 12 & GUELPH & 64.46 & 69 & CALIFORNIA IRVINE & 49.69 \\
\hline 13 & MCMASTER & 64.18 & 70 & WISCONSIN & 48.88 \\
\hline 14 & COLORADO STATE & 64.05 & 71 & HAWAII & 48.82 \\
\hline 15 & SUNY-BUFFALO & 63.30 & 72 & MINNESOTA & 48.71 \\
\hline 16 & MARYLAND & 63.23 & 73 & DUKE & 48.47 \\
\hline 17 & VIRGINIA TECH & 63.07 & 74 & TEXAS & 47.99 \\
\hline 18 & DELAWARE & 63.00 & 75 & RUTGERS & 47.90 \\
\hline 19 & SASKATCHEWAN & 61.97 & 76 & CALIFORNIA SANTA BARBARA & 46.51 \\
\hline 20 & NEW MEXICO & 61.24 & 77 & IOWA & 46.31 \\
\hline 21 & SOUTH CAROLINA & 61.02 & 78 & TENNESSEE & 46.22 \\
\hline 22 & PURDUE & 60.42 & 79 & BRIGHAM YOUNG & 45.75 \\
\hline 23 & CONNECTICUT & 60.22 & 80 & FLORIDA & 45.71 \\
\hline 24 & COLORADO & 60.18 & 81 & COLUMBIA & 45.31 \\
\hline 25 & WESTERN ONTARIO & 59.96 & 82 & ROCHESTER & 45.22 \\
\hline 26 & MIT & 59.81 & 83 & PENNSYLVANIA & 44.78 \\
\hline 27 & QUEEN'S & 59.43 & 84 & TEXAS TECH & 44.48 \\
\hline 28 & DARTMOUTH & 59.12 & 85 & CORNELL & 44.44 \\
\hline 29 & MONTREAL & 59.03 & 86 & VIRGINIA & 42.78 \\
\hline 30 & KENTUCKY & 58.93 & 87 & RICE & 42.75 \\
\hline 31 & ARIZONA & 58.18 & 88 & OREGON & 42.27 \\
\hline 32 & PENNSYLVANIA STATE & 57.92 & 89 & ILLINOIS URBANA & 42.10 \\
\hline 33 & LOUISIANA STATE & 57.84 & 90 & INDIANA & 39.91 \\
\hline 34 & WATERLOO & 56.97 & 91 & CALIFORNIA RIVERSIDE & 39.66 \\
\hline 35 & TEMPLE & 56.90 & 92 & PRINCETON & 39.60 \\
\hline 36 & YORK & 56.77 & 93 & NORTH CAROLINA STATE & 39.05 \\
\hline 37 & MICHIGAN STATE & 56.12 & 94 & MICHIGAN & 37.68 \\
\hline 38 & NOTRE DAME & 55.93 & 95 & GEORGETOWN & 37.12 \\
\hline 39 & NEW YORK & 55.92 & 96 & SUNY-ALBANY & 36.27 \\
\hline 40 & SOUTHERN ILLINOIS & 55.87 & 97 & TORONTO & 35.95 \\
\hline 41 & SUNY-STONY BROOK & 55.46 & 98 & BOSTON & 35.51 \\
\hline 42 & MCGILL & 55.25 & 99 & LOUISVILLE & 34.89 \\
\hline 43 & CALIFORNIA SAN DIEGO & 55.13 & 100 & NEBRASKA & 34.43 \\
\hline 44 & LAVAL & 54.64 & 101 & JOHNS HOPKINS & 32.67 \\
\hline 45 & CINCINNATI & 54.47 & 102 & CHICAGO & 32.58 \\
\hline 46 & TULANE & 54.46 & 103 & OKLAHOMA & 32.52 \\
\hline 47 & GEORGIA & 54.42 & 104 & CALIFORNIA BERKELEY & 32.42 \\
\hline 48 & WASHINGTON U.-ST. LOUIS & 54.40 & 105 & CALIFORNIA LOS ANGELES & 32.41 \\
\hline 49 & HOWARD & 54.29 & 106 & NORTH CAROLINA & 31.55 \\
\hline 50 & CALIFORNIA DAVIS & 54.07 & 107 & EMORY & 30.28 \\
\hline 51 & SYRACUSE & 53.84 & 108 & UTAH & 26.03 \\
\hline 52 & TEXAS A\&M & 53.59 & 109 & HARVARD & 24.20 \\
\hline 53 & BOSTON COLLEGE & 53.55 & 110 & GEORGE WASHINGTON & 23.02 \\
\hline 54 & BROWN & 53.49 & 111 & YALE & 22.06 \\
\hline 55 & SOUTHERN CALIFORNIA & 53.35 & & KENT STATE & $\mathrm{UA} / \mathrm{NA}$ \\
\hline 56 & ARIZONA STATE & 53.06 & & OKLAHOMA STATE & $\mathrm{UA} / \mathrm{NA}$ \\
\hline 57 & NORTHWESTERN & 52.94 & & & \\
\hline
\end{tabular}


Rank Order Table 21: Library InVestment Index

\begin{tabular}{|c|c|c|c|c|c|}
\hline RANK & INSTITUTION & VAlue & RANK & INSTITUTION & VALUE \\
\hline 1 & HARVARD & 5.9282 & 58 & OKLAHOMA & -0.2700 \\
\hline 2 & YALE & 3.5761 & 59 & NEW MEXICO & -0.2706 \\
\hline 3 & TORONTO & 2.6322 & 60 & TEMPLE & -0.2913 \\
\hline 4 & COLUMBIA & 2.3283 & 61 & ROCHESTER & -0.3052 \\
\hline 5 & CALIFORNIA, BERKELEY & 1.9283 & 62 & KANSAS & -0.3307 \\
\hline 6 & CALIFORNIA, LOS ANGELES & 1.5697 & 63 & CALIFORNIA, IRVINE & -0.3580 \\
\hline 7 & MICHIGAN & 1.5697 & 64 & WESTERN ONTARIO & -0.3790 \\
\hline 8 & PENNSYLVANIA STATE & 1.2839 & 65 & MANITOBA & -0.3817 \\
\hline 9 & TEXAS & 1.2685 & 66 & KENTUCKY & -0.3867 \\
\hline 10 & PRINCETON & 1.2024 & 67 & LAVAL & -0.4053 \\
\hline 11 & CORNELL & 1.1635 & 68 & COLORADO & -0.4069 \\
\hline 12 & ALBERTA & 1.1556 & 69 & BOSTON & -0.4211 \\
\hline 13 & NEW YORK & 1.0760 & 70 & SUNY-BUFFALO & -0.4232 \\
\hline 14 & WISCONSIN & 0.9566 & 71 & WAYNE STATE & -0.4402 \\
\hline 15 & NORTH CAROLINA & 0.8980 & 72 & CINCINNATI & -0.4485 \\
\hline 16 & WASHINGTON & 0.8770 & 73 & CALIFORNIA, DAVIS & -0.4956 \\
\hline 17 & MINNESOTA & 0.8355 & 74 & BROWN & -0.5108 \\
\hline 18 & ILLINOIS, URBANA & 0.7944 & 75 & BOSTON COLLEGE & -0.5198 \\
\hline 19 & INDIANA & 0.7750 & 76 & QUEEN'S & -0.5291 \\
\hline 20 & DUKE & 0.6941 & 77 & SOUTH CAROLINA & -0.5342 \\
\hline 21 & PENNSYLVANIA & 0.6635 & 78 & ILLINOIS, CHICAGO & -0.5475 \\
\hline 22 & OHIO STATE & 0.6597 & 79 & CALIFORNIA, SANTA BARBARA & -0.5613 \\
\hline 23 & SOUTHERN CALIFORNIA & 0.6293 & 80 & LOUISVILLE & -0.5620 \\
\hline 24 & VIRGINIA & 0.5021 & 81 & HOUSTON & -0.5646 \\
\hline 25 & BRITISH COLUMBIA & 0.5009 & 82 & DARTMOUTH & -0.5725 \\
\hline 26 & MCGILL & 0.4890 & 83 & DELAWARE & -0.5883 \\
\hline 27 & TEXAS A\&M & 0.4788 & 84 & IOWA STATE & -0.5947 \\
\hline 28 & CHICAGO & 0.4678 & 85 & SASKATCHEWAN & -0.6189 \\
\hline 29 & EMORY & 0.3154 & 86 & HAWAII & -0.6390 \\
\hline 30 & PITTSBURGH & 0.2854 & 87 & RICE & -0.6393 \\
\hline 31 & RUTGERS & 0.2612 & 88 & MCMASTER & -0.6761 \\
\hline 32 & ARIZONA & 0.2148 & 89 & SYRACUSE & -0.6966 \\
\hline 33 & MONTREAL & 0.1708 & 90 & OKLAHOMA STATE & -0.7032 \\
\hline 34 & CALIFORNIA, SAN DIEGO & 0.1360 & 91 & MISSOURI & -0.7032 \\
\hline 35 & NORTHWESTERN & 0.1317 & 92 & ALABAMA & -0.7121 \\
\hline 36 & JOHNS HOPKINS & 0.1299 & 93 & OREGON & -0.7430 \\
\hline 37 & FLORIDA & 0.0621 & 94 & FLORIDA STATE & -0.7476 \\
\hline 38 & IOWA & 0.0315 & 95 & MASSACHUSETTS & -0.7705 \\
\hline 39 & NORTH CAROLINA STATE & 0.0172 & 96 & NEBRASKA & -0.7898 \\
\hline 40 & WASHINGTON U.-ST. LOUIS & -0.0054 & 97 & SOUTHERN ILLINOIS & -0.8170 \\
\hline 41 & BRIGHAM YOUNG & -0.0125 & 98 & WATERLOO & -0.8190 \\
\hline 42 & MIAMI & -0.0177 & 99 & COLORADO STATE & -0.8248 \\
\hline 43 & ARIZONASTATE & -0.0352 & 100 & SUNY-STONY BROOK & -0.8345 \\
\hline 44 & YORK & -0.0769 & 101 & CALIFORNIA, RIVERSIDE & -0.8355 \\
\hline 45 & CONNECTICUT & -0.0962 & 102 & LOUISIANA STATE & -0.8441 \\
\hline 46 & GEORGETOWN & -0.0990 & 103 & WASHINGTON STATE & -0.8573 \\
\hline 47 & MICHIGAN STATE & -0.1348 & 104 & TULANE & -0.8577 \\
\hline 48 & PURDUE & -0.1384 & 105 & VIRGINIA TECH & -0.9120 \\
\hline 49 & MARYLAND & -0.1518 & 106 & CASE WESTERN RESERVE & -0.9157 \\
\hline 50 & MIT & -0.1583 & 107 & GUELPH & -0.9392 \\
\hline 51 & VANDERBILT & -0.1614 & 108 & GEORGIA TECH & -0.9923 \\
\hline 52 & TEXAS TECH & -0.1666 & 109 & SUNY-ALBANY & -0.9999 \\
\hline 53 & GEORGIA & -0.2119 & 110 & $\mathrm{OHIO}$ & -1.0118 \\
\hline 54 & NOTRE DAME & -0.2190 & 111 & AUBURN & -1.0380 \\
\hline 55 & TENNESSEE & -0.2278 & 112 & KENT STATE & -1.0547 \\
\hline 56 & UTAH & -0.2423 & 113 & HOWARD & -1.1500 \\
\hline 57 & GEORGE WASHINGTON & -0.2629 & \multicolumn{3}{|c|}{ Ranking as of August 12, 2009.} \\
\hline
\end{tabular}




\section{ARL Statistics Questionnaire, 2007-2008}

\section{INSTRUCTIONS FOR COMPLETING THE QUESTIONNAIRE}

\section{General Instructions}

Definitions of statistical categories can be found in NISO Z39.7-2004, Information Services and Use: Metrics \& statistics for libraries and information providers--Data Dictionary (http://www.niso.org/). ARL has been modifying the interpretation of the standard definitions to address questions posed by library staff at various member institutions that complete the survey and with feedback from the ARL Statistics and Assessment Committee (http://www.arl.org/stats/program/ meeting.html).

Please do not use decimals. All figures should be rounded to the nearest whole number.

Please respond to every question. If an exact figure cannot be provided, use NA/UA to indicate that the figure is either unavailable or not applicable. If the appropriate answer is zero or none, use $\mathbf{0 .}$

Although the form allows for data to be entered from both main and branch campuses, an effort should be made to report figures for the main campus only. (The US National Center for Education Statistics, Integrated Postsecondary Education Data System (IPEDS) defines a branch institution as "a campus or site of an educational institution that is not temporary, is located in a community beyond a reasonable commuting distance from its parent institution, and offers organized programs of study, not just courses.") If figures for libraries located at branch campuses are reported, please specify which branch libraries are included and which ones are excluded in the notes below.

A branch library is defined as an auxiliary library service outlet with quarters separate from the central library of an institution, which has a basic collection of books and other materials, a regular staffing level, and an established schedule. A branch library is administered either by the central library or (as in the case of some law and medical libraries) through the administrative structure of other units within the university. Departmental study/reading rooms are not included.

The questionnaire assumes a fiscal year ending June 30, 2008. If your fiscal year is different, please indicate this in the notes below by adjusting the reporting period.

Footnotes. Explanatory footnotes will be included with the published statistics. Provide any notes you may have in the footnotes area at the end of the survey. Reporting libraries are urged to record there any information that would clarify the figures submitted in that line, e.g., the inclusion and exclusion of branch campus libraries. Please make an effort to word your footnotes in a manner consistent with notes appearing in the published report, so that the ARL Office can interpret your footnotes correctly.

\section{Specific Instructions}

Question 1. Volumes in Library. Use the ANSI/NISO Z39.7-2004 definition for volume as follows:

a single physical unit of any printed, typewritten, handwritten, mimeographed, or processed work, distinguished from other units by a separate binding, encasement, portfolio, or other clear distinction, which has been cataloged, classified, and made ready for use, and which is typically the unit used to charge circulation transactions. Either a serial volume is bound, or it comprises the serial issues that would be bound together if the library bound all serials.

Include duplicates and bound volumes of periodicals. For purposes of this questionnaire, unclassified bound serials arranged in alphabetical order are considered classified. Exclude microforms, maps, nonprint materials, and uncataloged items. If any of these items cannot be excluded, please provide an explanatory footnote

Include government document volumes that are accessible through the library's catalogs regardless of whether they are separately shelved. "Classified" includes documents arranged by Superintendent of Documents, CODOC, or similar numbers. "Cataloged" includes documents for which records are provided by the library or downloaded from other 
sources into the library's card or online catalogs. Documents should, to the extent possible, be counted as they would if they were in bound volumes (e.g., 12 issues of an annual serial would be one or two volumes). Title and piece counts should not be considered the same as volume counts. If a volume count has not been kept, it may be estimated through sampling a representative group of title records and determining the corresponding number of volumes, then extrapolating to the rest of the collection. As an alternative, an estimate may be made using the following formulae:

\section{2 documents pieces per foot \\ 10 "traditional" volumes per foot \\ 5.2 documents pieces per volume}

Include e-book units, as long as these e-books are owned or leased and have been cataloged by your library. Include electronic books purchased through vendors such as NetLibrary ${ }^{\circledR}$ or Books $24 \times 7$, and e-books that come as part of aggregate services. Include individual titles of e-book sets that are treated as individual reference sources. Include locally digitized electronic books and electronic theses and dissertations. Provide a footnote explaining how many e-books you are reporting, preferably by specifying the products and the number of titles in a note.

Include volumes purchased collectively where the cost is shared at the time of purchase.

\section{If either formulas or sampling are used for deriving your count, please indicate in a footnote.}

Question 1b. Volumes Added. Include only volumes cataloged, classified, and made ready for use. Include government documents if they have been included in the count of volumes on line 1a. Do not include as part of Volumes Added Gross any government documents or other collections (such as large gift collections or e-book packages) that were added to the collection as the result of a one time download or addition to the OPAC. Include these items in Volumes Held of the previous year (Line 1a) and provide a footnote explaining the revision of Line 1a.

Question 2. Monographic Volumes Purchased. Report number of volumes purchased; do not include volumes received or cataloged. Include all volumes for which an expenditure was made during 2007-2008, including volumes paid for in advance but not received during the fiscal year. Include monographs in series and continuations. Include e-books that fit the NetLibrary ${ }^{\circledR}$ model, i.e., electronic manifestations of physical entities and/or units; provide a footnote explaining how many e-books you are reporting, preferably by specifying the products and the number of titles. If only number of titles purchased can be reported, please report the data and provide an explanatory footnote.

Question 3: Basis of Volume Count. A physical count is a piece count; a bibliographic count is a catalog record count.

Questions 4-5. Serials. Use the following definition adapted from AACR2 for a serial:
A bibliographic resource issued in a succession of discrete parts, usually bearing numbering, that has no predetermined conclusion. Examples of serials include journals, magazines, electronic journals, continuing directories, annual reports, newspapers, and monographic series.

Report the total number of unique serial titles, NOT SUBSCRIPTIONS, that you currently acquire and to which you provide access. Do not include duplicate counts of serial titles. Report each title once, regardless of how many subscriptions or means of access you provide for that title. Exclude unnumbered monographic and publishers' series. Electronic serials acquired as part of a bundle or an aggregated package should be counted at the title level, even if they are not cataloged, as long as the title is made accessible directly by the library (e.g., through a finding aid). If access is provided only through the overall platform or aggregator, do not report the individual titles but count the package as a single title.

Question 4a. Serial titles currently purchased. In the case of consortial agreements, count under 'serial titles currently purchased' those titles for which the library pays any amount from its budgeted expenditures. Include all titles that are part of bundles or aggregated packages, even if your library makes a partial payment for access to those titles. If a purchased title includes electronic access to the title, count that title ONLY ONCE (DEDUPED) as electronic only. If a database includes full-text and abstracted titles, the number of full-text titles can be counted. 
Question 4b. Serial titles: Not Purchased. Report other titles that your library receives and does not pay for directly under 'serial titles received but not purchased.' These titles may include exchanges, gifts, etc.

If serial titles have been purchased through a consortium whose budget is centrally funded and independent from the library's budget, these serials should be reported under 'serial titles currently received but not purchased.' If within a purchased or aggregated package it cannot be determined that some titles are not purchased, report all titles as purchased.

Freely accessible titles are those your library provides direct access to via cataloging records or through online serial lists of other finding aids.

To the extent possible, report all government document serials separately in $4 \mathrm{~b} . \mathrm{iv}$.

If separate counts of non-purchased and purchased serial titles are not available, report only the total number of serial titles currently purchased and received on line 4 , and report U/A for lines $4 \mathrm{a}$ and $4 \mathrm{~b}$.

Question 6. Microforms. Report the total number of physical units: reels of microfilm, microcards, and microprint and microfiche sheets. Include all government documents in microform; provide a footnote if documents are excluded.

Question 7. Government documents. Report the total number of physical units (pieces) of government documents in paper format that have not been counted elsewhere. Include local, state, national, and international documents; include documents purchased from a commercial source if shelved with separate documents collections and not counted above. Include serials and monographs. To estimate pieces from a measurement of linear feet, use the formula 1 foot $=52$ pieces and indicate in a footnote that the count is based on this estimate. Exclude microforms and non-print formats such as maps or CD-ROMs. Adjust line 1a, i.e., last year's Volumes Held, and provide a footnote if you are adding records to the OPAC for government documents previously held but not counted as part of Volumes Held (line 1a).

Question 8. Computer files. Include the number of pieces of computer-readable disks, tapes, CD-ROMs, and similar machine-readable files comprising data or programs that are locally held as part of the library's collections available to library clients. Examples are US Census data tapes, sample research software, locally mounted databases, and reference tools on CD-ROM, tape or disk. Exclude bibliographic records used to manage the collection (i.e., the library's own catalog in machine-readable form), library system software, and microcomputer software used only by the library staff.

Question 9. Manuscripts and archives. Include both manuscripts and archives measured in linear feet.

Question 10. Cartographic materials. Include the numbers of pieces of two- and three-dimensional maps and globes. Include satellite and aerial photographs and images.

Question 11. Graphic materials. Include the number of pieces of prints, pictures, photographs, postcards, slides, transparencies, film strips, and the like.

Question 12. Audio materials. Include the number of pieces of audiocassettes, phonodiscs, audio compact discs, reel-to-reel tapes, and other sound recordings.

Question 13. Film and video materials. Include the number of pieces of motion pictures, videocassettes, video laser discs, and similar visual materials.

Questions 14-20. Expenditures. Report all expenditures of funds that come to the library from the regular institutional budget, and from sources such as research grants, special projects, gifts and endowments, and fees for service. (For question 17, include non-library funds; see instruction Q17.) Do not report encumbrances of funds that have not yet been expended. Canadian libraries should report expenditures in Canadian dollars. (For your information, if interested in determining figures in US dollars, divide Canadian dollar amounts by 1.0101, the average monthly noon exchange rate published in the Bank of Canada Review for the period July 2007-June 2008.) Please round figures to the nearest dollar.

Question15a. Monographs. Report expenditures for volumes purchased counted on line 2. 
Question 15b. Serial titles. Report expenditures for serial titles counted on line 4a. Exclude unnumbered monographic and publishers' series, and encumbrances.

Question 15c. Other library materials. Include expenditures for all materials not reported in Questions 15a and 15b, e.g., backfiles of serials, charts and maps, audiovisual materials, manuscripts, etc. If expenditures for these materials are included in lines $15 \mathrm{a}$ and/or 15b and cannot be disaggregated, please report U/A and provide a footnote. Do not include encumbrances.

Question 15d. Miscellaneous expenditures. Include any other materials funds expenditures not included in questions 15a-c, e.g., expenditures for bibliographic utilities, literature searching, security devices, memberships for the purposes of publications, etc. Please list categories, with amounts, in a footnote. Note: If your library does not use materials funds for non-materials expenditures-i.e., if those expenditures are included in "Other Operating Expenditures" — report 0, not $\mathbf{U} / \mathbf{A}$, on line $15 \mathrm{~d}$.

Question 16. Contract Binding. Include only contract expenditures for binding done outside the library. If all binding is done in-house, state this fact and give in-house expenditures in a footnote; do not include personnel expenditures. (This figure should also be reported in the 2007-2008 ARL Preservation Survey, question 7b.)

Questions 17. Salaries and wages. Exclude fringe benefits. If professional and support staff salaries cannot be separated, enter $\mathbf{U} / \mathbf{A}$, on lines $17 \mathrm{a}$ and $17 \mathrm{~b}$ and enter total staff on line 17 .

Question 17c. Salaries and wages: Student Assistants. Report 100\% of student wages regardless of budgetary source of funds. Include federal and local funds for work study students.

Question 19. Other operating expenditures. Exclude expenditures for buildings, maintenance, and fringe benefits.

Questions 21-25. Electronic expenditures. These items are intended to indicate what portion of your institution's total library expenditures are dedicated to electronic resources and services. Please use the Footnotes to indicate any electronic materials expenditures you believe not to be covered by these questions. Many expenditures recorded in these questions should have been included in Question 20, total library expenditures.

Question 21. One-time electronic resource purchases. Report expenditures that are not current serials (i.e. are nonsubscription, one-time, or monographic in nature) for software and machine-readable materials considered part of the collections. Examples include periodical backfiles, literature collections, one-time costs for JSTOR membership, etc. Expenditures reported here may be derived from any of the following categories: Monographs (Q15a), Other Library Materials (Q15c), Miscellaneous (Q15d), or Other Operating Expenditures (Q19).

Question 22. Ongoing electronic resource purchases. Report subscription expenditures (or those which are expected to be ongoing commitments) for serial publications whose primary format is electronic and for online searches of remote databases such as OCLC FirstSearch, DIALOG, Lexis-Nexis, etc. Examples include paid subscriptions for electronic journals and indexes/abstracts available via the Internet, CD-ROM serials, and annual access fees for resources purchased on a "one-time" basis, such as literature collections, JSTOR membership, etc. Not all items whose expenditures are counted here will be included in Serial titles currently received (Question 4) or Serials Expenditures (Question 15b).

Q23. Bibliographic Utilities, Networks, and Consortia. Because it is increasingly common for ARL Libraries to enter into consortial arrangements to purchase access to electronic resources, both "Library" and "External" expenditure blanks and instructions are provided. Please use a footnote to describe expenditures that you believe are not covered by the question, or situations that do not seem to fit the instructions.

Q23a. From internal library sources. Report expenditures paid by the Library for services provided by national, regional, and local bibliographic utilities, networks, and consortia, such as OCLC and RLG, unless for user database access and subscriptions, which should be reported in Questions 21 or 22. Include only expenditures that are part of Other Operating Expenditures (Q19).

Q23b. From external sources. If your library receives access to computer files, electronic serials or search services through one or more centrally-funded system or consortial arrangements for which it does not pay fully and/or directly (for example, funding is provided by the state on behalf of all members), enter the amount paid by external bodies on its

98 · ARL Statistics 2007-2008 
behalf. If the specific dollar amount is not known, but the total student FTE for the consortium and amount spent for the academic members are known, divide the overall amount spent by your institution's share of the total student FTE.

Q24. Computer hardware and software. Report expenditures from the library budget for computer hardware and software used to support library operations, whether purchased or leased, mainframe or microcomputer, and whether for staff or public use. Include expenditures for: maintenance; equipment used to run information service products when those expenditures can be separated from the price of the product; telecommunications infrastructure costs, such as wiring, hubs, routers, etc. Include only expenditures that are part of Other Operating Expenditures (Q19).

Q25. Document Delivery/Interlibrary Loan. Report expenditures for document delivery and interlibrary loan services (both borrowing and lending). Include fees paid for photocopies, costs of telefacsimile transmission, royalties and access fees paid to provide document delivery or interlibrary loan. Include fees paid to bibliographic utilities if the portion paid for interlibrary loan can be separately counted. Include only expenditures that are part of Miscellaneous Materials Expenditures (Q15d) or Other Operating Expenditures (Q19), and only for those ILL/DD programs with data recorded in Questions 34-35.

Questions 26. Personnel. Report the number of FTE (full-time equivalent) staff in filled positions, or positions that are only temporarily vacant. ARL defines temporarily vacant positions as positions that were vacated during the fiscal year for which ARL data were submitted, for which there is a firm intent to refill, and for which there are expenditures for salaries reported on line 17.

Include cost recovery positions and staff hired for special projects and grants, but provide an explanatory footnote indicating the number of such staff. If such staff cannot be included, provide a footnote. To compute full-time equivalents of part-time employees and student assistants, take the total number of hours per week (or year) worked by part-time employees in each category and divide it by the number of hours considered by the reporting library to be a full-time work week (or year). Round figures to the nearest whole numbers.

Question 26a. Professional Staff. Since the criteria for determining professional status vary among libraries, there is no attempt to define the term "professional." Each library should report those staff members it considers professional, including, when appropriate, staff who are not librarians in the strict sense of the term, for example computer experts, systems analysts, or budget officers.

Question 26b. Support Staff. Report the total FTE (see instruction 26) of staff not included in $26 a$.

Question 26c. Student Assistants. Report the total FTE (see instruction Q26) of student assistants employed on an hourly basis whose wages are paid from funds under library control or from a budget other than the library's, including federal work-study programs. Exclude maintenance and custodial staff.

Question 27. Number of staffed library service points. Count the number of staffed public service points in the main library and in all branch libraries reported in this inventory, including reference desks, information desks, circulation, current periodicals, reserve rooms, reprographic services (if staffed as a public facility), etc. Report the number of designated locations, not the number of staff.

Question 28. Number of weekly public service hours. Report an unduplicated count of the total public service hours per typical full-service week (i.e., no holidays or other special accommodations) across both main library and branches using the following method (corresponds to IPEDS): If a library is open from 9:00 a.m. to 5:00 p.m. Monday through Friday, it should report 40 hours per week. If several of its branches are also open during these hours, the figure remains 40 hours per week. Should Branch A also be open one evening from 7:00 p.m. to 9:00 p.m., the total hours during which users can find service somewhere within the system becomes 42 hours per week. If Branch B is open the same hours on the same evening, the count is still 42 , but if Branch B is open two hours on another evening, or remains open two hours later, the total is then 44 hours per week. Exclude 24-hour unstaffed reserve or similar reading rooms. The maximum total is 168 (i.e., a staffed reading room open 7 days per week, 24 hours per day).

Questions 29-30. Instruction. Sampling based on a typical week may be used to extrapolate TO A FULL YEAR for Questions 29 and 30. Please indicate if responses are based on sampling. 
Question 29. Presentations to Groups. Report the total number of sessions during the year of presentations made as part of formal bibliographic instruction programs and through other planned class presentations, orientation sessions, and tours. If the library sponsors multi-session or credit courses that meet several times over the course of a semester, each session should be counted. Presentations to groups may be for either bibliographic instruction, cultural, recreational, or educational purposes. Presentations both on and off the premises should be included as long as they are sponsored by the library. Do not include meetings sponsored by other groups using library meeting rooms. Do not include training for library staff; the purpose of this question is to capture information about the services the library provides for its clientele. Please indicate if the figure is based on sampling.

Question 30. Participants in Group Presentations. Report the total number of participants in the presentations reported on line 29. For multi-session classes with a constant enrollment, count each person only once. Personal, one-to-one instruction in the use of sources should be counted as reference transactions on line 31. Please indicate if the figure is based on sampling. Use a footnote to describe any special situations.

Question 31. Reference Transactions. Report the total number of reference transactions. A reference transaction is an information contact that involves the knowledge, use, recommendations, interpretation, or
instruction in the use of one or more information sources by a member of the library staff. The term
includes information and referral service. Information sources include (a) printed and nonprinted
material; (b) machine-readable databases (including computer-assisted instruction); (c) the library's
own catalogs and other holdings records; (d) other libraries and institutions through communication
or referral; and (e) persons both inside and outside the library. When a staff member uses information
gained from previous use of information sources to answer a question, the transaction is reported
as a reference transaction even if the source is not consulted again.

If a contact includes both reference and directional services, it should be reported as one reference transaction. Include virtual reference transactions (e.g., e-mail, WWW form, chat). Duration should not be an element in determining whether a transaction is a reference transaction. Sampling based on a typical week may be used to extrapolate TO A FULL YEAR for Question 31. Please indicate if the figure is based on sampling.

EXCLUDE SIMPLE DIRECTIONAL QUESTIONS. A directional transaction is an information contact that facilitates the logistical use of the library and that does not involve the knowledge, use, recommendations, interpretation, or instruction in the use of any information sources other than those that describe the library, such as schedules, floor plans, and handbooks.

Questions 32-33. Circulation. For Question 32, count the number of initial circulations during the fiscal year from the general collection for use usually (although not always) outside the library. Do not count renewals. Include circulations to and from remote storage facilities for library users (i.e., do not include transactions reflecting transfers or stages of technical processing). Count the total number of items lent, not the number of borrowers.

For Question 33, report total circulation for the fiscal year including initial transactions reported on line 32 and renewal transactions. Exclude reserve circulations; these are no longer reported.

Questions 34-35. Interlibrary Loans. Report the number of requests for material (both returnables and non-returnables) provided to other libraries on line 34 and the number of filled requests received from other libraries or providers on line 35. On both lines, include originals, photocopies, and materials sent by telefacsimile or other forms of electronic transmission. Include patron-initiated transactions. Exclude requests for materials locally owned and available on the shelves or electronically. Do not include transactions between libraries covered by this questionnaire.

Questions 36. PhD Degrees. Report the number awarded during the 2007-2008 fiscal year. Please note that only the number of $\underline{\mathrm{PhD}}$ degrees are to be counted. Statistics on all other advanced degrees (e.g., DEd, DPA, MD, JD) should not be reported in this survey. If you are unable to provide a figure for PhDs only, please add a footnote.

Question 37. PhD Fields. For the purposes of this report, PhD fields are defined as the specific discipline specialties enumerated in the US Department of Education's Integrated Postsecondary Education Data System (IPEDS) "Completions"

$100 \cdot$ ARL Statistics 2007-2008 
Survey. Although the IPEDS form requests figures for all doctoral degrees, only fields in which $\underline{\mathrm{PhDs}}$ are awarded should be reported on the ARL questionnaire. Any exceptions should be footnoted.

Question 38. Instructional Faculty. Instructional faculty are defined by the US Department of Education as

members of the instruction/research staff who are employed full-time as defined by the institution, including faculty with released time for research and faculty on sabbatical leave.

Full-time counts generally exclude faculty who are employed to teach fewer than two semesters, three quarters, two trimesters, or two four-month sessions; replacements for faculty on sabbatical leave or leave without pay; faculty for preclinical and clinical medicine; faculty who are donating their services; faculty who are members of military organizations and paid on a different pay scale from civilian employees; academic officers, whose primary duties are administrative; and graduate students who assist in the instruction of courses. Please be sure the number reported, and the basis for counting, are consistent with those for 2006-2007 (unless in previous years faculty were counted who should have been excluded according to the above definition). Please footnote any discrepancies.

Questions 39-42. Enrollment. US libraries should use the Fall 2007 enrollment figures reported to the Department of Education on the Integrated Postsecondary Education Data System survey. Please check these figures against the enrollment figures reported to ARL last year to ensure consistency and accuracy. Note: In the past, the number of part-time students reported was FTE; the number now reported to IPEDS is a head count of part-time students. Canadian libraries should note that the category "graduate students" as reported here includes all post-baccalaureate students.

\section{FOOTNOTES}

Please consult the data entry Web interface (www.arlstatistics.org) for a copy of last year's footnotes. These can be found under "Data Repository" after you login into www.arlstatistics.org. Explanatory footnotes will be included with the published statistics. Reporting libraries are urged to record in the footnote section any information that would clarify the figures submitted, e.g., the inclusion and exclusion of branch campus libraries (see the "General Instructions" for definition of branch campus libraries). Please make an effort to word your footnotes in a manner consistent with notes appearing in the published report, so that the ARL Office can interpret your footnotes correctly.

Submit the completed questionnaire

by January 30, 2009.

For assistance, please e-mail Martha Kyrillidou (martha@arl.org) or Les Bland (les@arl.org)

Tel. (202) 296-2296. 


\section{ARL StATISTICS WorKSHEET 2007-2008}

This worksheet is designed to help you plan your submission for the 2007-2008 ARL Statistics. The figures on this worksheet should be similar to those in the "Summary" page of your web form, except in cases where data are unavailable. If an exact figure is unavailable, use "NA/UA". If the appropriate answer is zero or none, use " 0. ."

Reporting Institution

Date Returned to ARL

Report Prepared by (name)

Title

E-mail address Phone number

Contact person (if different)

Title

E-mail address Phone number

PAGE ONE - VOLUMES

1. Volumes held June $30,2008(1 a+1 b)$

(1)

1a. Volumes held June 30, 2007

(1a)

1b. Volumes added during the year (1b.i - 1b.ii)

(i) Volumes added - Gross

(1b.i)

(ii) Volumes withdrawn during year

(1b.ii)

2. Number of monographic volumes purchased

(2)

3. Basis of volume count is:

(3) Physical Bibliographic 
4. Total number of serial titles currently received, including periodicals $(4 . a+4 . b)(4)$

4a. Number of serial titles currently purchased $(4 a . i+4 a . i i)$

(4a)

4a.i Electronic

(4a.i)

4a.ii Print (and other format) serials purchased (4a.ii)

4b. Number of serial titles currently received but not purchased

$$
(4 b . i+4 b . i i+4 b . i i i+4 b . i v)
$$

4b.i Consortial

4b.ii Freely accessible

4b.iii Print (and other format) - Exchanges, gifts, etc.

4b.iv Government documents

5. Government documents are included in count of Current Serials?

\section{OTHER LIBRARYMATERIALS}

\section{Microform units}

7. Government documents not counted elsewhere

8. Computer files

9. Manuscripts and archives (linear ft.)

\section{AUDIOVISUAL MATERIALS}

10. Cartographic

(10)

11. Graphic

12. Audio

13. Film and Video (4b.i)

(4b.ii)

(4b.iii)

(4b.iv)

(5) Yes No

\author{
(6)
}

(7)

(8)

(9) 
PAGE THREE - EXPENDITURES

14. Are the below figures reported in Canadian dollars?

(14)

Yes No

15. Total Library Materials Expenditures $(15 a+15 b+15 c+15 d)$

(15)

15a. Monographs

(15a)

15b. Serial titles, including periodicals

15c. Other Library Materials

(15c)

15d. Miscellaneous

16. Contract binding

(16)

17. Total Salaries and Wages $(17 a+17 b+17 c)$

17a. Professional staff

(17a)

17b. Support staff

17c. Student assistants

18. Fringe benefits are included in expenditures for salaries and wages?

(18)

Yes No

19. Other operating expenditures

(19)

20. Total library expenditures

$$
(15+16+17+19)
$$

\section{ELECTRONIC MATERIALS EXPENDITURES}

21. One-time electronic resource purchases

22. Ongoing electronic resource purchases (e.g., subscriptions, annual license fees)

23. Bibliographic Utilities, Networks, and Consortia

23a. From internal library sources

23b. From external sources

(23b)

24. Computer hardware and software

25. Document Delivery/Interlibrary Loan 
PERSONNEL (Round figures to nearest whole number.)

26. Total Staff FTE $(26 a+26 b+26 c)$

26a. Professional staff, FTE

26b. Support staff, FTE

26c. Student assistants, FTE (26a)

(26b)

(26c)

\section{STAFFED SERVICE POINTS AND HOURS}

27. Number of staffed library service points

(27)

28. Number of weekly public service hours

\section{INSTRUCTION}

29. Number of library presentations to groups

(29)

29a. Is the library presentations figure based on sampling?

(29a) Yes No

30. Number of total participants in group presentations reported in line 29

30a. Is the total participants in group presentations

(30a) Yes No figure based on sampling?

\section{REFERENCE}

31. Number of reference transactions

(31)

31a. Is the reference transactions figure based on sampling?

(31a) Yes No 
CIRCULATION

32. Number of initial circulations (excluding reserves)

33. Total circulations (initial and renewals, excluding reserves)

\section{INTERLIBRARYLOANS}

34. Total number of filled requests provided to other libraries

35. Total number of filled requests received from other libraries or providers

\section{PhD DEGREES AND FACULTY}

36. Number of PhDs awarded in FY2007-2008

37. Number of fields in which PhDs can be awarded

38. Number of full-time instructional faculty in FY2007-2008

ENROLLMENT - FALL 2007

(Line numbers refer to IPEDS survey form.)

39. Full-time students, undergraduate and graduate

(Add line 8, columns $15 \mathcal{E} 16$, and line 14, columns $15 \mathcal{E} 16$. .)

40. Part-time students, undergraduate and graduate

(Add line 22, columns $15 \& 16$, and line 28, columns 15 \& 16.)

41. Full-time graduate students (Line 14, columns $15 \mathcal{E} 16$.

42. Part-time graduate students (Line 28 , columns 15 \& 16.)

\section{FOOTNOTES}

On the Web form, you will be able to add footnotes to individual questions, as well as footnotes that apply to your entire institution. Please provide any information which would clarify the figures submitted, e.g., the inclusion of branch campus libraries or any special projects which might cause radical increases or decreases. Please use the footnotes in the ARL Statistics 2006-2007 for comparison if necessary. Please consult the data entry Web interface (www.arlstatistics.org) for a copy of last year's footnotes. These can be found under "Historical Data" by clicking a survey's arrow icon under 
the "View Summary" column and then selecting the "Footnotes" tab. Please make an effort to word your footnotes in a manner consistent with notes appearing in the published report, so that the ARL Office can interpret your footnotes correctly.

Submit the completed questionnaire by January 30, 2009.

For assistance, please e-mail Martha Kyrillidou (martha@arl.org) or Les Bland (les@arl.org) Tel. (202) 296-2296. 



\section{FOOTNOTES}

Footnotes may also include errata and corrections to data from prior years not previously reported. Numbers refer to columns in Library Data Tables and to Questionnaire numbers. Unless otherwise stated all figures are as of 06/30/2008.

QUESTION FOOTNOTE

NUMBER

\section{ALABAMA}

All figures are as of 09/30/2008.

Library branches included: main, law, and health sciences libraries.

1a Volumes held June 30, 2007 revised to 2,844,387. 5,643 added to historical count due to retrospective government document cataloging; health sciences had a difference in 2006-2007 volumes held 9/30/07 (17,857) and 2007-2008 volumes held 9/30/07 (20,791) due to adjustments to problem records in ILS.

2

5,821 e-books included in monographic volumes purchased.

Number increased significantly from 2006-2007 due to new ARL counting procedures and better local tracking in Serials Solutions Knowledgebase. De-duplicated using best practices procedures distributed by ARL for Serials Solutions. De-duplicated using ISSNs and other identification as described in best practices document. For the electronic titles, there were 30,598 without ISSNs.

$4 \mathrm{a}$

The number of serials titles currently purchased $(44,089)$ is composed of (4ai) electronic: 41,705 and (4aii) print: 2,384. Both (4ai) and (4aii) were changed to UA/NA so that the operating system could aggregate other figures from the Alabama Law and Health Sciences surveys.

4b The number of serials titles currently received but not purchased $(19,034)$ is composed of (4bii) freely accessible: 17,518 and (4biii) print: 1,516. Both (4bii) and (4biii) were changed to UA/NA so that the operating system could aggregate other figures from the Alabama Law and Main library surveys.

4bi, 4bii Separate numbers of consortial and freely available not available.

15d Includes Center for Research Libraries (CRL) membership.

19

Includes bibliographic utilities, memberships (except CRL), and literature searching.

ALBERTA

All figures are as of 03/31/2008.

Library branches included: Health Sciences Library and the Law Library are included.

1

Includes e-Book titles.

1a

Includes e-Book titles held at the end of March 2007.

Past figures were inaccurately reported. Precise number of monographs purchased from 2000-01 onward is unavailable; all monographs purchased are included in gross volumes added.

$4-4 \mathrm{~b} \quad$ Includes government documents.

$7 \quad$ This data is included in microform units.

15-25 All expenditures were reported in Canadian Dollars. Expenditures as reported in Canadian dollars: (15a) \$6,148,532; (15b) $\$ 10,258,161$; (15c) 0 (15d) \$643,927; (15) \$17,050,620 (16) \$83,550; (17a) \$7,279,475; (17b) $\$ 10,266,485 ;$ (17c) $\$ 1,126,258 ;(17) \$ 18,672,218 ;(19)$ $\$ 11,286,901 ;$; (20) \$47,093,289; (21) \$7,430,459; (22) \$7,237,813; (23a) 0 (23b) 0 (24) 0 (25) 0.

15c Cannot be disaggregated from monograph expenditures (15a) and serial expenditures (15b).

15d Includes GST (Goods and Services Tax), postage, courier, and supplies expenditures, as well as membership expenditures not already paid through the materials budget.

$17 \quad$ Excludes fringe benefits.

21 This figure includes over 5 million of funding provided for digital library content.

Figure based on actual hours worked. Prior to 2000-2001, figures were based on hours hired to work.

Now excludes all renewals.

Now includes renewals done over the internet-- these were not included in years prior to 2007-2008.

We were unable to provide this figure for 2003-2004. We can now provide it again effective with the 2004-2005 report.

This figure can vary from year to year. 


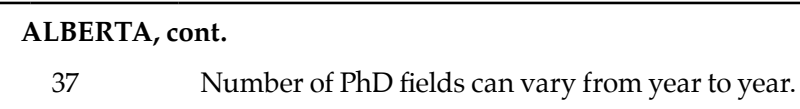

\section{ARIZONA}

Library branches included: Main, Science Engineering, and Fine Arts.

1a

Volumes held June 30, 2007 revised to 5,702,736.

\section{ARIZONA STATE}

Library branches included: ASU Tempe, ASU W
1a Volumes held June 30, 2007 revised to 4,351,495.

3 Only the Polytechnic library portion (which is 895) is a bibliographic count.

7 2006-2007 figure revised to 659,603.

$8 \quad 2006-2007$ figure revised to 7,169.

35 2006-2007 figure revised to $43,636$.

42 2006-2007 figure revised to $4,920$.

AUBURN

All figures are as of 09/30/2008.

Library branches included: Charles Allen Cary Veterinary Medical Library; Library of Architecture, Design and Construction.

BOSTON

All figures are as of 06/30/2008.

Library branches included: Mugar (main), Theology, and Special Collections libraries.

1a

Volumes held June 30, 2007 revised to 2,755,000.

\section{BOSTON COLLEGE}

Library branches included: John J Burns Library of Rare Books and Special Collections, Bapst Library, School of Social Work Library and the Educational Resource Center.

1a Volumes held June 30, 2007 revised to 2,487,141.

15c Significant purchases of backfiles and manuscript collection account for 2007-2008's higher number.

25 Amount submitted for 2006-2007 was not accurate. This year's data (2007-2008) are accurate.

\section{BRIGHAM YOUNG}

All figures are as of 12/31/2007.

4bi Given the nature of our reporting period, an attempt will be made to include this in the next year's (2008-2009) statistical survey. For now, this figure may be lumped into (4bii) \& (4biii).

29 The increase in groups and participants reflects expanding the count to capture not just traditional library instruction courses, but all other library sponsored presentations as defined in the instructions.

\section{BRITISH COLUMBIA}

All figures are as of 03/31/2008.

Library branches included: Applied Sciences Reading Room, Archaeology Reading Room, Architecture Reading Room, Art + Architecture + Planning library, Art History Visual Resources Centre, Asian Library, Biomedical Branch Library, B'nai Brith Reading Room , Botanical Gardens Reading Room , Coolie Verner Reading Room, Crane Library, David Lam Library, Education Library, Geographic Information Centre, Hamber Library, ICICS/Computer Science Reading Room, Irving K. Barber Learning Centre, Koerner Library, Law Library, LERC Reading Room, Museum of Anthropology Reading Room, Music Library, Okanagan Library, Psychiatry Reading Room, Rare Books \& Special Collections, Regent College John Richard Allison Library, Robson Square Library, Science \& Engineering Division, St. Mark's College Library, St. Paul's Hospital Library, TAG Reading Room, TRIUMF

Library, University Archives, Vancouver School of Theology Library, Womens' Resources Centre, Womens' Studies Reading Room, Woodward Library, Xwi7xwa Library (First Nations House of Learning).

The Irving K. Barber Learning Centre opened in spring 2008; the UBC Okanagan campus continued to grow.

1 Includes 284,711 e-books.

1bi Includes 23,096 new e-books.

4 Current serial titles de-duped (purchased/not purchased). 


\section{BRITISH COLUMBIA, cont.}

4ai Cannot provide "received but not purchased" serials information.

4aii Print serials: decrease in subscriptions (reflects Transition to Online program); serial titles are de-duped.

8 Increase in numeric datafiles; e-databases.

11 Graphic materials increased because of gifts to University Archives and Art History Visual RC collections.

13 Film/video materials increased because of gifts to University Archives and Art History Visual RC collections.

15-25 Expenditures as reported in Canadian dollars: (15a) \$3,329,815; (15b) \$10,509,335; (15c) \$841,156; (15d) \$387,589; (15) \$15,067,895; (16) $\$ 137,000$; (17a) $\$ 8,585,798 ;(17 b) \$ 6,739,533 ;$; 17 c) $\$ 842,870 ;(17) \$ 16,168,201 ;(19) \$ 3,995,503 ;(20) \$ 35,368,599 ;(21) \$ 178,426 ;(22) \$ 7,547,413$; (23a) \$345,394; (23b) \$0; (24) \$327,019; (25) \$144,007.

15a Amount decrease because of reallocation to serials.

15b Decreased due to transition to online program (print cancellations) and prepayments in $2006-2007$.

16

Decreased serial binding because of the "Transition to Online" program for serials.

17b There were no signing bonus expenditures for support staff in 2007-2008.

17c Salaries for student assistants: rose because of increased staff at UBC Okanagan campus; increase in cooperative education programs and increase in services for the opening of the new Barber Learning Centre.

19 Includes additional expenditures for opening of new Barber Learning Centre.

20 Total library expenditures show decrease in budget. See footnotes for (15) and (17b).

21 One time computer files purchase figure includes expenditures for e-books.

Decreases in Document Delivery/ILL expenditures because of consortial agreements and increased online access.

26b Increase in support staff at UBC Okanagan.

26c Increase in student assistant staff because of cooperative education programs, UBC Okanagan, and the opening of the Barber Learning Centre.

$27 \quad$ Closed 2 branches: MacMillan and Math branches.

$42 \quad$ UBC Okanagan growth continuing in graduate students.

BROWN

Library branches included: Rockefeller Library, Sciences Library, John Hay Library, Orwig Music Library, Art Slide Library, Library Collections Annex, John Carter Brown Library.

Medical library statistics cannot be disaggregated from the main because the medical collection is an integral part of the Sciences Library.

1a Volumes held June 30, 2007 revised to 3,742,950.

4a Increase represents upgrade in electronic resources (e.g., Business Source Complete), changes to aggregate resources, and the purchase of individual new journal titles.

15a Increase represents primarily changes in JCB purchases.

16 Decrease due to stopping the binding of shelf-ready receipts.

32 Corrected 2006-2007 figure: 198,849. Corrected 2005-2006 figure: 221,448.

33 Corrected 2006-2007 figure: 301,692. Corrected 2005-2006 figure: 302,892.

40-42 Normal fluctuation in part-time students enrolled.

\section{CALIFORNIA, BERKELEY}

Volumes held June 30, 2007 revised to 10,897,766. This total includes Tier 1 e-book volumes purchased collectively with University of California Partner Libraries: 473,819 (Tier 1: Acta Sanctorum, Literature Online, Oxford Scholarship Online, Early English books Online, Eighteenth Century Collections Online, Safari Tech Books, Schillers Werke). Additionally, the Berkeley campus e-book volumes total 73,857 (Tier 3: ebrary and netLibrary selections, World Bank E Library, Humanities/History E Books Project, Oxford Scholarship Online Special Subjects, Congressional Hearings Retrospective A). The Berkeley campus has independently acquired 113, 396 e-book volumes (ebrary selections, netLibrary selections, World Ban Elibrary, Humanities/History E Books Project, Oxford Scholarship Online Special Subjects, Congressional Hearing Retrospective A). 


\section{CALIFORNIA, BERKELEY, cont.}

3 We used a combination of both physical and bibliographic counts to respond to this question. The source of our information for supplying volume counts has historically been a physical count created by aggregating annual incremental reports by library unit managers. However, these last 2 years (2006-2008) e-books were added to the question, and we used a bibliographic count for these. Counts for e-books acquired through collective purchase are generated by our system-wide cataloging division. Counts for e-books acquired through independent campus-level purchase are derived from our local inventory managed by the Electronic Resources Librarian through an Electronic Resource Finder (ERF).

4bi-4biv Our method for generating a total number for serials relies upon a review of our OPAC. We were able to de-duplicate holdings for multiple print titles, and holdings in which we had both an open holding statement for electronic and print. We are unable to distinguish paid for and free active titles with our current method of generating the data so we entered the totals in (4a) and (4b) and left (4b) subtotals UA/NA.

\section{CALIFORNIA, DAVIS}

1a Volumes held June 30, 2007 revised to 3,602,106.

1bi Previous reporting did not include e-books that are part of the collection and cataloged locally.

1bii Extensive weeding was conducted in the 2006-2007 reporting period.

4-4ai A revised methodology for counting purchased serial titles was used for the 2007-2008 reporting period.

4b-4biii A revised methodology for counting non-purchased serial titles was used for the 2007-2008 reporting period.

\section{CALIFORNIA, IRVINE}

1a This figure was revised to 2,973,408. Per ARL instructions, this figure includes 195,487 e-books that were added during 2007-2008.

\section{CALIFORNIA, LOS ANGELES}

1a

Volumes held June 30, 2007 revised to 8,282,525.

4 This figure is repeated from the previous year (2006-2007). Because of the process of identifying a process to dedupe and capture data in compliance with the new definition and due to this transition we are publishing the same figure as last year (2006-2007). UCLA has not yet determined accurate counts for subscription titles that are obtained without cost via the internet, since these titles do not have order records in UCLA's bibliographic database. Because there is a significant increase in these titles, UCLA is devoting efforts to capturing this information in a systematic and reliable way for the next annual statistical report. Number of full-time faculty has increased to 1,948 .

\section{CALIFORNIA, RIVERSIDE}

The rationale for the significant difference in serials statistics for the University of California, Riverside Libraries for 2007-2008 is: a) an increase in serial titles resulting from consortial co-investments; b) an increase in the number of government documents serial titles that have been entered into our cataloging records.

\section{CALIFORNIA, SAN DIEGO}

Library branches included: Arts Library, Center for Library \& Instructional Computing Services, International Relations \& Pacific Studies Library, Mandeville Special Collections Library, Science \& Engineering Library, Scripps Institution of Oceanography Library, Social Sciences \& Humanities Library.

Biomedical Library and Medical Center Library are included in the ARL Survey for Health Sciences Libraries.

1 E-book figures are not cataloged into a specific library since they are not site specific. Their numbers are all reported on the Main Library Survey, Geisel Library.

1a Volumes held June 30, 2007 revised to 3,125,499.

17 Increase in salaries is due to several high salaried positions created and filled, coupled with a reduction in turnover of positions.

17c Although student FTE decreased, actual expense increased due to the minimum wage increase, additional duty increases, and the addition of a Student Security Unit.

28 Weekly public service hours extends to 168 during finals week each quarter when we do have a staffed library open 24 hours, 7 days a week.

31 Includes 4,412 virtual reference transactions.

\section{CALIFORNIA, SANTA BARBARA}

28 2006-2007's figure multiplied the number of weekly public service hours times the number of service hours for each service point, making it inaccurate. 2007-2008's figure reflects the actual number of weekly public service hours.

\section{CASE WESTERN RESERVE}

Library branches included: the Mandel School of Applied Social Sciences- Harris Library. 


\section{CASE WESTERN RESERVE, cont.}

1a Volumes held June 30, 2007 revised to 2,735,459. An adjustment was made to the Volumes Held 6/30/07 figure to include e-books that have been added to the collection (including: Early American Imprints, Series I and II; EEBO, EEBO-TCP, Safari Tech Books Online and Reference resources).

9 This number includes the University Archives, which now reports to the Kelvin Smith Library (Main Library).

15d This expenditure includes: memberships for ARL, SPARC, CNI, CLIR, OhioLINK and ICPSR. Also included is ARL Conference on Diversity, ARL LibQUAL+® Survey and theses charges from ProQuest.

\section{CENTER FOR RESEARCH LIBRARIES}

$17 \mathrm{a}$

$26 b$

$26 \mathrm{c}$

\section{CHICAGO}

1

1a

2

4

$4 \mathrm{~b}$

\section{CINCINNATI}

All University of Cincinnati Libraries located on its central campus are included.

Two branch libraries on two-year college campuses, Raymond Walters College and Clermont College, are not included in the ARL statistics.

The increase in Professional salaries from 2006-2007 to 2007-2008 was mainly due to the addition of a few higher paying jobs, along with internal promotions (from support staff to professional staff).

Total FTE was 18.5 but question requires an integer. In order to have a total of 61 FTE this figure was rounded down.

Total FTE was 12.5, but question requires an integer. In order to have a total of $61 \mathrm{FTE}$, this figure was rounded up.

Includes 851,880 electronic monographs.

Volumes held 30 June 2007 revised to 8,351,863. Increase due in part to addition of MARC records for monographic titles available in databases and addition of MARC records for USGPO electronic monographs.

Includes 36,414 electronic monographs purchased in 2007-2008; does not include monographs for which we previously had access and had counted as added, but added MARC records for in 2007-2008.

This increase (over 2006-2007) can be attributed to a growth in available free e-journals. This is due to an expansion in the number of available and cataloged free e-journals and the addition of MARC records for USGPO online documents.

This increase can be attributed to the addition of MARC records for government documents (almost 11,000) and an addition to the number of freely accessible journals added to our catalogs (about 5,000).

Excludes unprocessed and partially processed items.

Includes standard ongoing operating costs only.

This is an estimated figure.

Effective 2007-2008, circulation figures exclude departmental charges that had previously been included. Had we not made this adjustment, the circulation figure would have been 458,057.

Includes 486 requests filled by the Center for Research Libraries.

In previous years we understood the request to cover only those full-time instructional/research staff with faculty status. In 20072008 we determined that we should count all full-time instructional/research staff without regard to faculty status. An additional 410 personnel were added as a consequence of our more inclusive definition.

Volumes held June 30, 2007 revised to 3,547,887. Adjustments: net adjustment is an adjustment of 129,067 volumes. 2,705 net Gov Docs adjustment-- 3,357 Government documents volumes cataloged retroactively; 652 volumes subtracted for Government documents cleanup. 166,278 volumes added for batch loads of e-resources (1 time, of 5,000 or more): ECCO, NBER, ChadwyckHealey LION. 39,916 volumes withdrawn in a lost, missing cleanup of stacks.

Figure is historically based upon a bibliographic count. Additions and deletions based on physical count.

Notes on the serial counts; the serial count is by title, deduped, in a "cascading hierarchy": (1) Paid (1st electronic, then other format); A large majority of the substantial number of serial titles received via our consortium are paid via an index figure based upon titles owned when the relationship with the publisher began (therefore we considered them all paid). (2) Not purchased (the not purchased titles are not separated by electronic vs. print and other formats, but by source). (3) The Not-purchased titles in the Freely-accessible and Government documents categories have very little overlap, because of the strategy used to construct that the serial list, and then were deduped; however, numbers here are only the titles which have not already been included in the Paid category. (4) The Freely-accessible titles include the titles marked as "free" from Serials Solutions, so long as those same titles had not already been recorded in the paid Serials Solutions titles. (5) Some Government document titles are also included in Paid Serials Solutions aggregates, and not-purchased Government document titles could also be included in the free Serials Solutions titles.

Government documents not counted elsewhere are smaller because of retrospective cataloging of documents.

Part of difference in salaries total caused by $\$ 88,000$ retirement payment made in 2006-2007 but not during 2007-2008. 


\section{CANADA INSTITUTE OF SCIENTIFIC AND TECHNICAL INFORMATION}

All figures are as of 03/31/2008.

1 We have been using a piece count, but as of 2007-2008 we will use assumed volume count of 10 volumes per foot, which will cut the collection to 1,688,187.

15-25 Expenditures as reported in Canadian dollars: (15a) \$524,029; (15b) \$10,685,356; (15c) \$304,704; (15d) \$11,810; (15) \$11,525,899; (16) $\$ 0 ;(17 a) \$ 0 ;(17 b) \$ 0 ;(17 c) \$ 0 ;(17) \$ ; 23,053,000$ (19) \$11,853,101; (20) \$46,432,000; (21) \$304,704; (22) \$1,980,558; (23a) \$0; (23b) \$0; (24) $\$ 2,276,904 ;(25) \$ 0$.

\section{COLORADO}

Library branches included: Business, Earth Sciences, Engineering, Math/Physics, Music.

\section{COLORADO, cont.}

1

2

4aii

10

11

$15 \mathrm{c}$

$15 \mathrm{~d}$

\section{COLORADO STATE}

1a Volumes held June 30, 2007 revised to 1,994,256. Library staff reevaluated the way e-books were being recorded.

2 Monographic volumes for 2006-2007 should have been 27,576.

4bi These are counted with the purchased titles.

7 Government Documents not appearing on the catalog are no longer counted.

$8 \quad$ Library has done a re-evaluation as to how computer files are recorded in the catalog.

9 Increase of donated materials-- both from the community and the campus.

\section{COLUMBIA}

Columbia data includes The Columbia Center for New Media Teaching and Learning (CCNMTL) and the Center for Digital Research and Scholarship (CDRS).

1 Includes 679,755 e-books for Butler Library and 65,203 e-books reported by Teachers College Library.

1a Volumes held June 30, 2007 revised to 10,004,848.

$2 \quad$ Includes 349,797 e-books.

4 Teachers College information serials information reflects unique titles; all other titles received by Teachers College overlap with, and are counted in, Butler Library.

15d Includes costs associated with maintenance of collection (e.g., shipping, supplies, relocation, memberships).

17c Additional graduate assistants and part-time positions were added to the library budget (Teachers College).

19 2006-2007 figure should have been \$1,013,450 (Teachers College).

20 2006-2007 figure should have been $\$ 3,841,617$ (Teachers College). 
COLUMBIA, cont.

Butler Library has a credit balance for 2007-2008 of \$42,757 but system will not accept a credit. The total for Columbia has been correctly submitted by adjusting other units -- correct totals for Columbia are: Butler \$42,757 (credits); Health Sciences \$8,609; Law $\$ 25,643$; Teachers College $\$ 38,541$ for a total expenditure of \$30,036 (note: subtract Health Sciences, Law and Teachers College from Butler's figure to get total expenditure). Barnard's numbers are included in Butler's report.

26c Figure for 2006-2007 should have been 16 (Teachers College).

32 2006-2007's figure included check-ins and check-outs. Check-outs should have been 45,072 (Teachers College).

33 2006-2007's figure included check-ins and check-outs. Check-outs and renewals should have been 66,173 (Teacher College).

35 All ILL requests initiated by Barnard are handled centrally by Columbia University.

\section{CONNECTICUT}

Library branches included: Music, Art, Pharmacy, Avery Point, Waterbury, Stamford, Torrington, Greater Hartford.

1a Volumes held June 30, 2007 revised to 3,964,901.

$1 \mathrm{~b} \quad$ Volumes held June 30, 2007 revised to 8,030,766. The main library undertook a project to eliminate materials no longer relevant to the institution's current curriculum.

1bi-1bii The main library undertook a project to eliminate materials no longer relevant to the institutions current curriculum.

4a-4aii Figures reported for 2006-2007 were incorrect. Methodology for de-duping and counting serial titles was revised according to ARL “Best Practice Documentation” for 2007-2008. Titles counts now accurately reflect UConn holdings. Ongoing commitment to convert print subscriptions to online format when feasible.

4b Figures reported for 2006-2007 were incorrect. Methodology for de-duping and counting serial titles was revised according to ARL "Best Practice Documentation" for 2007-2008, title counts now accurately reflect UConn holdings.

4biii Ongoing commitment to convert print subscriptions to online format when feasible.

Less physical materials to bind as a result of an increase in online and digital collections.

17 Slight decrease in FTE being reported for this time period. Fringe benefits are no longer being included in the salary expenditure figures, this accounts for the decrease in expenditure being reported between 2006-2007 and this reporting period (2007-2008)

17c Slight increase in FTE being reported for this time period. Increase in federal minimum wage scale has had an impact on student payroll.

19 Increased cost for goods and services for this reporting period (2007-2008).

Limited purchasing of electronic backfiles for 2007-2008.

Increased emphasis on electronic access to materials.

This figure reflects an increase in funding for equipment.

Increased expenditure in IMF fees as borrowing requests increase.

Library is engaged in an effort to reduce number of participants in each presentation in order to facilitate a more effective learning environment.

31 The number of users seeking out reference assistance has reduced. The number of library staffed hours at a designated reference service point have been reduced.

Information discovery tools are expanding and improving resulting in our users requesting additional sources, many of which are esoteric in nature, for their research that are not available from the libraries' exisiting collections.

Figures demonstrate an increase in applications and enrollment.

CORNELL

Library branches included: the Adelson, Africana, Annex, Asia Collections, Engineering, Fine Arts, Hotel, Industrial \& Labor Relations, Rare \& Manuscript Collections, Law, Management, Mann/Entomology, Mathematics, Music, Olin, Physical Sciences, Uris, and Veterinary Libraries on the Ithaca campus, and the New York Agricultural Experiment Station Library in Geneva NY.

Excludes the Medical College Library in New York, NY.

1a Volumes held June 30, 2007 revised to 8,030,766. Change in volumes held June 30, 2007 reflects the fact that additional e-books were counted. Includes all e-books with records in the online catalog.

4 The count was calculated before the 2007-2008 definition was released, following the 2006-2007 definition. This is an estimate only. 8,700 were estimated to be non-current e-tittles. Includes numbered and unnumbered monographic series. Law print count includes non-serial continuations.

Figure is in cubic feet. 
CORNELL, cont.

15a Most integrating resource expenditures are included in monographic expenditures.

15d This figure reflects shipping costs only.

17a For libraries other than Law, includes expenditures for librarians only. For Law, includes expenditures for librarians and "exempt" staff.

22 Includes an estimate for amount spent on electronic subscriptions for large publishers providing both print and electronic whose payment records are coded as print only.

26 Does not include staff in positions that were temporarily vacant. Includes professional, support and student staff on grants and projects.

26a For libraries other than Law, includes librarians only. For Law, includes librarians and "exempt" staff.

28 Includes 27.5 security-staffed hours (only study space and computer lab available). 2006-2007's figure should have been 146.

31a Data is from 12 random sample weeks (same as 2006-2007).

32-33 Includes ILL lending and some staff transactions.

\section{DARTMOUTH}

Library branches included: Feldberg Business \& Engineering Library, Kresge Physical Sciences Library, Paddock Music Library, Sherman Art Library, Rauner Special Collections Library and College Archives.

1a Volumes held June 30, 2007 revised to 2,732,828.

Count of serials titles was made using a different source this year (2007-2008), which we believe is more accurate.

11 Reduction due to large discard project.

40, 42 Part-time enrollment is primarily non degree-seeking students. Undergraduate degree-seeking students are full-time by requirement. Graduate arts and sciences program part-time enrollment varies widely from year to year.

\section{DELAWARE}

1a Volumes held June 30, 2007 revised to 2,741,215.

$4 \quad$ ARL's new methodology that counts unique titles was fully implemented for the 2007-2008 statistics.

9 Excludes the University Archives, which are not administered by the Library.

32 Library's automated system does not differentiate between initial loans and renewals.

DUKE

1a Volumes held June 30, 2007 revised to 5,849,608.

1b-1bii Increase due to contrast with 2006-2007's data. In 2006-2007 the Medical Center Library and the Ford Business Library withdrew many volumes.

$4,4 \mathrm{~b} \quad$ Increased numbers for 2007-2008 are due to improved data compilation in this area.

4bii The increase is due to this data being unavailable in 2006-2007. This is an estimate based on SFX data.

4biii Increase due to improved data compilation in this area for 2007-2008.

12 Increase due to increased collecting in this area.

15c Decreased figures due to decreased spending in this area.

17a Increase due to increased expenditures in this area.

19 Increase due to increased expenditures in this area including a computer hardware "refresh" for all staff members in the main library.

21 Decrease apparent because there was an increase in funding in this area due to Strategic Initiative Project funding supporting a one time purchase of electronic backfiles of serials.

The main library adopted an e-only exceptions policy for serials. The policy is available here: http://library.duke.edu/about/ collections/eonly.html. This adoption resulted in transferring many serial subscriptions from print to electronic. library. 
DUKE, cont.

29-30 Decrease due to decreased participation in this area in 2007-2008.

35 Increase due to the creation of a shared consortia catalog available here, http://search.trln.org/, resulting in increased borrowing between Triangle Research Libraries Network institutions.

36 Increase due to normal fluctuation in this area.

EMORY

1a

4ai

FLORIDA

1a

\section{FLORIDA STATE}

Includes all Gainesville, Florida campus libraries: Marston (Science), Architecture/Fine Arts, Journalism, Music, Education, Law, and Health Science Center. Also includes the Health Science library facility in Jacksonville, Florida.

Includes the Health Sciences Center, Hugh MacMillan (Law), Oxford College, and Pitts Theology libraries, as well as libraries at Grady Hospital and the Medical School.

Volumes held June 30, 2007 revised to 3,296,754.

This was reported incorrectly for Main Library in 2006-2007. Should have reported 15,288.

In 2007-2008, this was paid by actual invoice rather than prepaying estimated invoice, and fund accounting practices also changed.

This was reported incorrectly for Theology Library in 2005-2006. Reported number was 19,850, but should have been 898 .

Faculty counts have varied because of Health Science Library practices associated with counts of clinical faculty, and whether separate Medical School faculty numbers have been accounted for in years prior to 2007-2008.

Numbers for 2005-2006 and 2006-2007 were over-reported. Main number now adjusted with totals for Health Sciences, Law, Oxford, and Theology to equal Registrar's Fall 2007 totals.

Numbers for 2005-2006 and 2006-2007 were over-reported. Because Health Sciences provides student count separately, requires main to subtract that number from Registrar's report to provide correct totals.

Volumes held June 30, 2007 revised to 4,230,703.

Library branches included: Goldstein College of Information Library; Allen Music Library; Pepper Center Library; Panama City, Panama; Panama City, Florida Library; Ringling Museum of Art Library.

1a Volumes held June 30, 2007 revised to 2,803,491. The number for 2007-2008 (1a) is different than the number for the 2006-2007 question (1) due to a mathematical error in the 2006-2007 report. The correct number for (1) in 2006-2007 is 2,989,700.

15c Increase in this category is due to the inclusion of previously excluded categories: Database access and e-books.

23b 2006-2007's number was based on an incorrect formula. The figure for 2006-2007 should have been 463,059.

29 This number is almost double 2006-2007's figure. This is due to the large increase in instruction sessions at the Main Library. Additionally, this is due to a much better relationship with partners and establishing new relationships with partners; especially the First Year Experience (FYE) for incoming students.

31 The drop in reference transactions is due to an error made in the 2006-2007 survey. That number included directional transactions which should not be included in that category. The correction was made in this survey.

36 The 2006-2007 figure is incorrect. It should be 631. That puts the increase within the normal range.

\section{GEORGE WASHINGTON}

Volumes held June 30, 2007 revised to 1,733,391. The figures were changed because of extensive collection weeding and reevaluation.

This figure reflects extensive collection weeding and re-evaluation.

In 2006-2007, only "firm order" books were included in the shelf ready statistics. "Approval books" were added 2007-2008. There was an increase in SPEC cataloging which was the result of several influxes of SPEC materials that required cataloging. The focus of computer files was shifted to SPEC.

The 2006-2007 total monographs purchased should be 19,543. (Not 27,928 as reported previously).

We gather this data from our SFX knowledge base. The increase in numbers purchased is due to a project that added foreign language (primarily Asian) serials. We have subscribed to many of these for several years, but were never available via SFX.

4aii This is a smaller number than 2006-2007 because of a project in 2007-2008 that cleaned up older purchase orders that had not yet been closed.

15c These are one-time purchases of databases and backfiles. In 2006-2007 we added this expenditure to monographs (15a). 


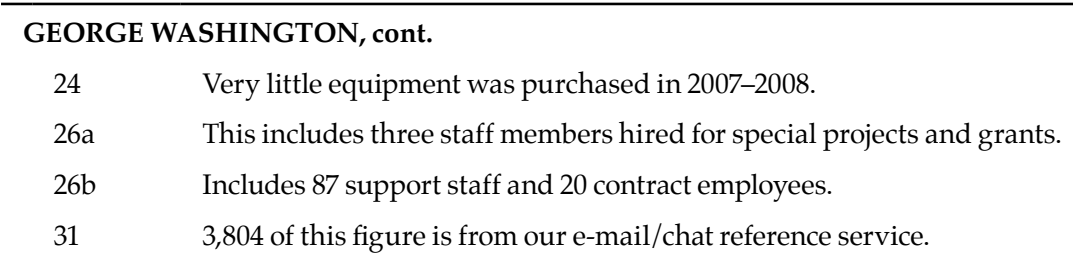

\section{GEORGETOWN}

Includes Woodstock Theological Center Library, Blommer Science Library, National Reference Center for Bioethics Literature, and SFS-Qatar Library.

4b The number of serials titles currently received, but not purchased is (4b) 9,045 and is composed of (4bi) Consortial: UA/NA; (4bii) Freely Accessible: 7,503; (4biii) Print (and other format): 534 and (4biv): Government Documents: UA/NA. All subtotals of (4b) were changed to UA/NA so that the operating system could aggregate the 1,008 serials reported in (4b) of the Georgetown Medical library survey.

\section{GEORGIA}

All figures are as of 07/31/2008.

Library branches included: the Law Library.

E-books held 167,810 (PAHO: 12; OECD: 2902; EEBO, EVANS, SABIN: 164,896; GALE: 10).

2

E-books purchased 195 (OECD: 185; GALE: 10).

15d Memberships \$95,366; bibliographic utilities \$114,585; shipping \& handling \$45,910; EndNote- RefWorks \$39,605; Media/ Maintenance \$56,815.

\section{GEORGIA TECH}

Includes the Architecture Library.

17a This number is significantly lower than in years prior to 2007-2008 as it reflects an attempt to more closely align it with the total staff count as reported in Question (26). Due to the campus accounting system, the number previously reported included some support staff.

17b This number is significantly higher than in years prior to 2007-2008 due to the change reported in footnote for (17a).

\section{GUELPH}

1a

1bi-1bii Figures are for print-only volumes.

15-25 Expenditures as reported in Canadian dollars: (15a) \$1,432,923; (15b) \$3,622,684; (15c) \$139,874; (15d) \$546,340; (15) \$5,741,821; (16) \$34,659; (17a) \$3,168,133; (17b) \$3,116,677; (17c) \$335,219; (17) \$6,620,029; (19) \$1,168,787; (20) \$13,565,296; (21) \$326,700; (22) \$3,374,454; (23a) $\$ 189,454 ;(23 b) \$ 272,901 ;(24) \$ 404,160 ;(25) \$ 82,568$.

19 Does not include travel expenses.

23a Includes payments to Canadian Research Knowledge Network $(\$ 20,000)$ and Ontario Council of University Libraries $(\$ 169,454)$.

23b Represents monies received from Federal and Provincial governments to fund Canadian Research Knowledge Network purchases. Increase in professional staff with expansion of Learning Commons programming. Previously reported 2006-2007 values were inaccurate; 179,824 is the correct figure. Previously reported 2006-2007 values were inaccurate; the correct figure is 450,645.

\section{HARVARD}

1a $15 \mathrm{c}$

HAWAII
Volumes held June 30, 2007 revised to 15,945,710.

Increase the result of both digitization projects and acquisition of special collections.

Increase the result of both digitization projects, for some research resources and acquisition of special collections.

Library branches included: University of Hawaii at Manoa Library (main) housed in two buildings: Hamilton Library, Sinclair Library. Also includes the Health Sciences Library, and William S. Richardson School of Law Library. 


\section{HAWAII, cont.}

$4 \mathrm{~b}$

10

17

$17 a$

HOUSTON

$1 b i$

4

$4 a i$

4aii

4 bi

4bii

16

\section{HOWARD}

1a

\section{ILLINOIS, CHICAGO}

Library branches included: Main Library, Health Sciences Library and Law Library.

Volumes held June 30, 2007 revised to 2,435,249.

Title count, according to new criteria, including those in aggregated databases, if the title has a record in the library OPAC.

Library branches included: Richard J. Daley Library, Science Library.

3 E-books count $(17,222)$ is bibliographic.

9 Includes statistics for health sciences branches.

16 Figure included in item (19), other operating expenditures.

19 Includes portion of (23), Bibliographic Utilities, Networks and Consortia: OCLC, annual memberships for CIC and CARLI.

23a Does not include OCLC, membership in CARLI or CIC, which are included in (19) (Other Operating Expenditures).

34-35 Figures unavailable for all institutions with which UIC has reciprocal borrowing privileges. I-Share data for health sciences branch lending are included. 


\section{ILLINOIS, URBANA}

$1 \mathrm{a}$

\section{INDIANA}

The Law Library was included in this years (2007-2008) ARL Statistics.

The Medical Library was not included.

1a Volumes held June 30, 2007 revised to 8,552,806.

1bi Includes corrected e-book count.

4

\section{IOWA STATE}

10

\section{JOHNS HOPKINS}

Library branches included: Statistical data from the Main Library and Veterinary Medical Library, plus three reading rooms (Design, Physical Sciences, and Math) are included in the Main Library figures.

Library branches included: Art, Biological Sciences, Business, Geoscience, Mathematical Sciences, Physics, Engineering, Music and Psychology libraries.

Volumes held June 30, 2007 revised to 4,791,330.

Includes programming staff that support libraries but not paid directly from libraries' budgets.

Including equipment costs that support libraries but not paid directly from libraries' budgets.

Including hard/software costs that support libraries but not paid directly from libraries' budgets.

\footnotetext{
Library branches included: Milton S. Eisenhower Library, Welch Medical Library, R.E. Gibson Library Applied Physics Laboratory, Bologna Center, Institute of the History of Medicine, Arthur Friedheim Library and J. Peabody Institute Archives, SAIS Mason Library, Washington Centers.

1a Volumes held 30 June 2007 revised to 3,724,594.

16 Amount reported for 2007-2008 in contract printing was understated by \$5,335 due to an error, which will be corrected in a subsequent reporting period.

\section{KANSAS}

1a Volumes held June 30, 2007 revised to 4,191,836.

4a Expansion of existing and acquisition of new licensed full-text databases.

$4 \mathrm{~b} \quad$ New process and method of reporting, so not comparable with years prior to 2007-2008.

15c 2007-2008 non-book materials: \$134K, one-time electronic resource purchases: $\$ 873 \mathrm{~K}$.

16 Amount reported for 2007-2008 in contract printing was overstated by $\$ 13,423$ due to recording error, which will be corrected in a subsequent reporting period.
}

\section{KENT STATE}

Included are Kent State branches (Ashtabula, Geauga, East Liverpool, Salem, Stark, Trumbull, and Tuscarawas) and campus libraries: Architecture, Chemistry/Physics, Fashion, Map, Mathematics/Computer science, Music.

4

$4 \mathrm{a}, 15 \mathrm{~b}$

29

Serial titles are not deduplicated.

The numbers reported represent an estimate and are not deduplicated. Serials have been difficult to deduplicate by title rather than by subscription since serials now come in multiple and differing formats. This estimate in serial titles has resulted in an increased number of serials, but a reduction in the expenditures for serials. Thus, our unit price for serials purchased may be unrealistically low.

Library orientation "tour" is now offered online, asynchronously, and is not included in this count. 
KENT STATE, cont.

303,547 additional students (not included in this number) participated in our online asynchronous library orientation.

\section{KENTUCKY}

1a Volumes held June 30, 2007 revised to 3,636,191. Revised total: 98,481 e-books added to June 30, 2007 total.

2 136,277 volumes of an ECCO e-books package were purchased during 2007-2008, but have not been included in this count.

4ai Total includes medical library e-journals.

4bi-4bii Includes law and medical serials.

4biv Unable to de-duplicate government documents total for varying formats.

42 This is a significant decrease from 2006-2007.

LAVAL

All figures are as of 05/31/2008.

There are no separate law and medical libraries. The data for these sections are included in the main library statistics.

15-25 Expenditures as reported in Canadian dollars: (15a) \$2,734,064; (15b) \$7,396,666; (15c) \$156,669; (15d) \$192,253; (15) \$10,479,652; (16) $\$ 77,024$; (17a) \$4,348,480; (17b) \$5,914,892; (17c) \$48,826; (17) \$10,312,198; (19) \$613,458; (20) \$21,482,332; (21) \$470,395; (22) \$5,255,210; (23a) $\$ 0 ;(23 b) \$ 0 ;(24) \$ 0 ;(25) \$ 55,177$.

25 Since VDX software installation, we have cancelled our subscription to two databases.

\section{LIBRARY \& ARCHIVES CANADA}

All figures are as of 31/03/2008.

1 No inventory has been conducted recently. The only data currently available is the total number of bibliographic notices (pieces). At this time, no estimate could be prepared to determine the corresponding number of volumes.

15-25 Expenditures as reported in Canadian dollars: (15a) \$782,529; (15b) \$454,764; (15c) \$449,652; (15d) \$134,131; (15) \$1,824,076; (16) \$0; (17a) $\$ 53,153,654 ;$; (17b) $\$ 23,344,623$; (17c) $\$ 712,012$; (17) $\$ 77,210,289 ;(19) \$ 0 ;(20) \$ 79,034,365 ;(21) \$ 0 ;(22) \$ 38,848 ;(23 a) \$ 0 ;(23 b) \$ 0 ;(24)$ $\$ 15,021,628 ;(25) \$ 11,307$.

\section{LIBRARY OF CONGRESS}

All figures are as of 09/30/2008.

Library branches included: Library Services (i.e. Library of Congress general and special-format collections); Law Library of Congress is included, and is so footnoted (in instances for which statistics cannot be separated).

Library branches not included: National Library Service for the Blind and Physically Handicapped.

1

$1 \mathrm{a}$

$1 b$

$4 \mathrm{bi}$

4 bii

4biii

4 biv

Figures are for volumes held as of Sept. 30, 2008. Total does not include 2,610,704 volumes held in the Law Library of Congress. Figure revised from 29,864,066 reported in 2006-2007 to 29,775,178.

This figure excludes Law Library of Congress.

Titles accessed via EZB consortium are counted with "freely accessible" in (4bii).

Includes titles to which the Library of Congress (LC) has access through its membership in the Elektronische Zeitumgen Bibliothek (EZB).

Includes serial titles forwarded to the LC permanent collections from the US Copyright Office, estimated at 7,000 in 2006-2007 (estimated because of copyright transition to new automated system).

Government documents serial are included in count of serial titles received but not purchased (4) (i.e. not counted separately).

Includes $\$ 73,500$ from materials funds to pay for bibliographic representatives in Greece, China, and West African countries; $\$ 165,800$ in payments to OCLC, Inc. for FirstSearch and reading room access to WorldCat; and $\$ 1,670$ FEDLINK fee (this amount is $7.75 \%$ of $\$ 21,554$, the portion of LC's FirstSearch expenditure that is paid through FEDLINK).

Includes binding contracts administered by the Library's overseas offices. For 2006-2007, LC figure should be corrected to 1,742,141.

Estimate pending final figures. Expenditure is expected to be lower than in 2006-2007, when an operating system upgrade of 5,100 Library workstations commenced.

Also reported under Miscellaneous Materials Expenditures (15d) and Bibliographic Utilities (23).

Permanent staff are reported as "Professional" or "Support staff" only within the Library Services service unit. All other staff are reported as "Professional," including financial staff, human resources specialists, and information technology staff.

Slight decrease from 2006-2007, when LC offered many tours during the ALA Annual Conference held in Washington, DC. Also, in 2007-2008 LC initiated the self-guided Library of Congress Experience. 


\section{LIBRARY OF CONGRESS, cont.}

31 Includes 545,084 reference transactions in the public reading rooms; 323,469 in the US Copyright Office; 650 directed to the Preservation Directorate; and 871,287 reference transactions conducted by the Congressional Research Service.

32 Includes 1,131,411 items circulated to researchers on the Library of Congress campus and 156,356 items circulated to congressional members and staff (the only category of users who may charge items for use off-campus). Does not include items charged for telework or other technical processing.

$34 \quad$ Includes 5,812 non-returnables.

\section{LOUISIANA STATE}

Library branches included: Health (Veterinary), Law Library and the Main Library.

1a Volumes held June 30, 2007 revised to 4,067,169. This number is revised from 2006-2007 to include 17,018 electronic books added in 2007-2008: Early American Newspapers, Series II and III, 1801-1819 (258); Early English Books Online (Pollard and Redgrave, STC I) (850); Early English Books Online (Wing, STC II) (3,750); Early English Books Online (Thomason Tracts) (2,127); Knovel (435); Naxos Music Library (4,574); Netlibrary Collection VI (3,638); Twentieth Century North American Drama $(1,386)$.

$1 \mathrm{~b}$

$1 \mathrm{bi}$

1 bii

2

$4 a$

4aii

$4 b$

6

7

8

9

10

12

13

$15 b$

26-26b

$26 \mathrm{c}$

40

\section{LOUISVILLE}

Included: Main Library, Medical Library, University Archives, Music Library, Art Library, and the Law Library.

The medical and law libraries data have been aggregated into the main library's figures. Louisville did not submit separate law and medical library surveys for this period (2007-2008).

Decrease is due to budget changes.

This number comes from cataloging, database management, documents, serials, and special collections.

This number comes from database management and documents.

This figure consists of 15,405 paper volumes plus 17,018 e-books.

The number of print serials was unavailable so the sub-questions of (4ai) and (4bi) have been marked UA/NA.

Since this number is unavailable, one cannot accurately calculate the average price per serial purchased for 2007-2008 (i.e., the serials unit price is unrealistically low).

The number of consortial serials for the main library was unavailable so this has been marked UA/NA on the main library survey.

This number is derived from 5,576 microforms (from database management, documents, serials, and Special collections) added to the total from 2006-2007.

This number comes from the LSU Documents Depository Library and it has decreased every reporting period during the last few years leading up to 2007-2008.

This number comes from the "Count Items" report for this item category. For reporting purposes, a reported unit of 1 is equal to 1 CD-ROM, 1 COMPDISK, and 1 DVD-ROM (this excludes the VetMed library).

This number is composed 113 linear feet of manuscripts (as determined by Special Collections) to which the total from 2006-2007 has been added.

This number is reported by the Cartographic Information Center (CIC). The "count items" report is determined by counting a unit of 1 equal to 1 MAP (excludes VetMed library).

This number is derived from the "count items report" with 1 equal to 1 AUDIO-CASS, 1 AUDIO-CD, and 1 LP (Long Play phonograph records) (excludes VetMed library).

This number comes from the "count items" report. For this item, 1 is equal to 1 DVD, 1 Film reel, 1 Video cassette, and 1 Videodisk (excludes VetMed library).

Since the number of print serials purchased is unavailable, one cannot accurately calculate the average price per serial purchased for this year (2007-2008).

This reflects changes in staffing patterns.

This reflects changes in staffing patterns. This number is an average of 117 bodies at any one time, divided by four (since most work 10 hours a week).

This figure demonstrates decreasing enrollment.

University of Louisville libraries serve as the central archive for the entire University of Louisville system. The reason for the large increase in the 2007-2008 manuscript collection figure is because the routine destruction of non-permanent university records has not been performed recently. Usually, non-essential materials are routinely destroyed every year, but this has not been done for the last several years because of staff shortages. 


\section{MANITOBA}

All figures are as of 03/31/2008.

Libraries included: William R. Newman Agriculture Library, Architecture and Fine Arts Library, Archives \& Special Collections, Elizabeth Dafoe Library, Fr. Harold Drake Library, St. John's College Library, Donald W. Craik Engineering Library, E.K. Williams Law Library, Albert D. Cohen Management Library, Eckhardt-Grammatte Music Library, Sciences and Technology Library, Neil John Maclean Health Sciences Library, Bill Larson Library, Carolyn Sifton-Helent Fuld Library, Concordia Hospital Library, J.W. Crane Memorial Library, Misericordia Health Centre Library, Riverview Health Centre Virtual Library, Seven Oaks General Hospital Library, and the Victoria General Hospital Library.

1bii Increase over 2006-2007 figure is due to intense weeding and collections maintenance in preparation for move of materials to newly constructed storage unit.

15-25 Expenditures as reported in Canadian dollars: (15a) $\$ 2,057,771 ;(15 b) \$ 5,880,826 ;(15 c) \$ 45,748 ;(15 d) \$ 430,585 ;(15) \$ 8,414,930 ;(16)$ $\$ 161,724 ;$ (17a) $\$ 5,390,193 ;$ (17b) \$5,762,765; (17c) \$719,113; (17) \$11,872,071; (19) \$1,817,932; (20) \$22,266,657; (21) \$1,080,416; (22) \$3,352,558; (23a) \$87,744; (23b) \$0; (24) \$284,729; (25) \$167,672.

2006-2007 figure should have been 28,787.

\section{MASSACHUSETTS}

Library branches included: Main (W.E.B. DuBois) Library, Integrated Sciences Library, and the Engineering Library.

1bi A very large purchase of e-books was made in 2006-2007 and as a result the gross volumes added for 2006-2007 was an anomaly.

4

$17 \mathrm{c}$

MCGILL

The total number of serial titles and each subcategory have been deduplicated according to ARL recommended techniques. The 2006-2007 unique serial counts were not accurate.

Expenditures for student assistants includes $\$ 62,246$ from federal work-study grants.

Includes 156 sessions taught by Teaching Assistants (TAs) who were trained by librarians.

Includes 3,759 students in sessions taught by TAs who had been trained by librarians.

Excludes 41 EdD degrees awarded by the School of Education.

All figures are as of 05/31/2008.

Library branches included: Birks Reading Room, Education Library, Islamic Studies Library, Life Sciences Library, Humanities \& Social Sciences Library, Blackader-Lauterman Library of Art \& Architecture, Marvin Duchow Musich Library, Howard Ross Library, Macdonald Campus Library (agricultural \& Environmental Sciences), Osler Library of the History of Medicine, Nahum Gelber Law Library, Schulich Library of Science \& Engineering, Edward Rosenthall Library of Mathematics \& Statistics, and the Walter Hitschfeld Geographic Informatic Centre.

Volumes held June 30, 2007 revised to 4,461,040. There are 357,195 e-books that are not cataloged, but are available to library users. They have not been added to this count.

Separate figures are not kept for consortial serials.

Slides and filmstrips are reported in (11).

Expenditures as reported in Canadian dollars: (15a) $\$ 5,263,342 ;(15 b) \$ 10,290,257 ;(15$ c) $\$ 247,793 ;(15 d) \$ 446,701 ;(15)$ \$16,248,093; (16) $\$ 248,762$; (17a) \$6,095,591; (17b) \$7,716,288; (17c) \$829,709; (17) \$14,641,588; (19) \$4,359,872; (20) \$35,498,315; (21) \$1,302,287; (22) \$7,674,494; (23a) $\$ 363,388$; (23b) $\$ 0 ;(24) \$ 524,983 ;(25) \$ 34,628$.

The number of faculty reported in 2006-2007 was incorrect. We include all of the medical faculty (affiliated doctors) who are associated with McGill's extensive teaching hospitals in our total faculty count.

\section{MCMASTER}

All figures are as of 04/30/2008.

Library branches included: Mills Memorial Library, H.G. Thode Library, and the Innis Library.

Corrections to the 2006-2007 McMaster Library Statistics: (1a) Volumes held as of June 30, 2006: 1,842,679; (1b) Volumes added: 12,503 (1bi) Volumes added gross: 16,518; (1bii)Volumes withdrawn: 4,015.

1a

Volumes held June 30, 2007 revised to 2,165,581.

1b This figure has e-books included.

$1 b i$

1 bii

Figure has 146,575 e-books included. Note: add 146,575 to 2006-2007 e-books purchased in 2007-2008.

6

Deduping project increased withdrawals figures.

Large amount of microfilm set sent to Nippising. 


\section{MCMASTER, cont.}

15-25 Expenditures as reported in Canadian dollars: (15a) \$1,069,128; (15b) \$5,771,863; (15c) \$523,071; (15d) \$981,321; (15) \$8,345,383; (16) $\$ 4,108 ;$; (17a) \$3,041,636; (17b) \$4,165,527; (17c) \$610,019; (17) \$7,817,182; (19) \$1,418,590; (20) \$17,585,263; (21) \$402,772; (22) \$4,953,432; (23a) \$96,055; (23b) \$0; (24) \$112,920; (25) \$95,696.

15a Increase because of e-books added to collection.

16 Decreased number of journals received.

26 Classroom Audio Visual staff of 9 moved to Mills Library.

MIAMI

All figures are as of 05/31/2008.

Includes the Richter Library (central library); Calder Memorial Library (medical library); law library; and the architecture, business, marine, and music libraries.

Includes 542,380 ETD (Electronic Theses and Dissertations) and e-books in the following collections: ebrary, NetLibrary, Early English Books Online, Gale's 18th Century Collections Online, Women Writers Project, Wright American Fiction, Making of the Modern World, Making of Modern Law, Literature Online, Sabin, Evans, Shaw-Shoemaker, and Gale Virtual Library.

Volumes purchased for 2006-2007 was 53,561, not 38,779 as reported (in prior years central library reported titles purchased, not volumes).

Medical library computer files are reported in (13).

13 Medical library audiovisual materials (10 through 13) and computer files (8) are reported in (13).

17a Excludes salaries of the University Librarian and directors of the law and medical libraries.

26a Includes library faculty and selected non-faculty professional staff. In prior years all non-faculty professional staff in the medical library were reported in (26a); in 2008 they are reported in (26b).

$26 \mathrm{~b} \quad$ Includes both support and selected non-faculty professional staff. In prior years all non-faculty professional staff in the medical library were reported in (26a); in 2008 they are reported in (26b).

28 Combined weekly public service hours for all libraries is 122.

31 Transactions are based on enumerations in central, business, medical, and music libraries, and sampling in marine and law libraries. Reference transactions for architecture library are not included.

\section{MICHIGAN}

Library branches included: University Library, Bentley Historical Library, William L. Clements Library, Kresge Business Administration Library, and the Law Library.

Does not include any libraries from the Dearborn or Flint UM (University of Michigan) campuses.

1a Volumes held June 30, 2007 revised to 9,075,625. This number does not agree with the "Volumes held June 30, 2007" reported in the 2006-2007 ARL statistics because it includes 421,820 e-books that were held at that time, but not included in the definition of the volume count for that year. Additionally, this includes 234,583 digitizations of volumes in the public domain (and hence available full text) that were added to the HathiTrust digital repository in 07-08 and which are accessible through our catalog.

1bi This number includes 129,433 physical volumes and as well as e-books added during the year $2007-2008$.

$4 \quad$ Owing to the way we keep our records, we cannot break down our serials into these categories while still de-duping by ISSN. Catalogued serials with ISSNs have been de-duped within and across libraries, and the duplication rate in this category was $3.6 \%$. The reported figure also includes 19,659 cataloged serials without ISSNs and 21,294 serials which have not been cataloged; neither of these categories have been de-duped.

6 We no longer count microform by physical unit. Our much recent count by that method was 8,445,189 reported in 2006-2007. This number remains reasonably accurate as we acquire very little material in this form anymore and we do not withdraw any of it.

15c Includes the total library expenditures for Clements Library, which are not sub-divided into the categories listed.

\section{MICHIGAN STATE}

Includes the following branch libraries: Biomedical \& Physical Sciences, Business, Engineering, Gull Lake, Mathematics, Veterinary Medical Center libraries.

1bii One branch library was closed.

10 Cartographic materials were counted incorrectly for 2006-2007.

4biv Includes 6,580 online titles.

15d Includes the following: $\$ 9,100.00$ for Portico (a journal archive membership); $\$ 4,000.00$ for Public Library of Science (Open Access membership); $\$ 41,500.00$ for Biomed Central (prepay account for Open Access article fees). 


\section{MINNESOTA}

University of Minnesota coordinate campuses are not included.

1a Volumes held June 30, 2007 revised to 6,778,001. Volume count adjusted to reflect adjustments to counts in special collections areas.

1biii Increase reflects weeding projects in various locations.

4a Used same counting algorithms as 2006-2007. Increase reflects new investments and improved "discoverability."

4bi Breakdown between consortial and freely accessible electronic serials is not available.

4biii Breakdown between print serials and print government documents is not available.

11 Breakdown by material type is not available. Total audio-visual items for all included libraries is 869,236.

15c Change reflects the fact that in 2006-2007 this category was high due to purchases of e-serial backsets.

15d Increase in use of collections funds for bibliographic utilities, interlibrary loan subsidy, search enhancements, etc.

25 Increase is due to more accurate reporting of costs in all departments. 2006-2007's figure was under-reported.

26 Decrease in staffing represents vacant positions, not reduction of available positions.

31 Significant decrease may be partly because of adoption of software for recording statistics leading to more accurate reporting and fewer estimates.

31a Counts from some service points may be estimated.

MISSOURI

Library branches included: Health Sciences Library, Vet Med Library, Math Library, Geology Library, Engineering Library, Journalism Library, Newspaper Library, Archives, and the Library Depository.

15b Of the $\$ 5,582,484$ reported here; $\$ 2,774,288$ is from the University-wide systems office for the four campus system. University of Missouri- Columbia (MU) has over $50 \%$ of the students, but we use all of these journals.

$19 \$ 412,774$ of this figure is for Online Cataloging system for all four campus Library Systems Offices.

22 This represents \$2,774,288 for four campus Library System Offices, and \$3,193,514 for MU Libraries Serials.

\section{MASSACHUSETTS INSTITUTE OF TECHNOLOGY}

All MIT Libraries are included in these statistics.

18 Fringe benefits are included.

MONTREAL

All figures are as of $05 / 31 / 2008$.

15-25 Expenditures as reported in Canadian dollars: (15a) $\$ 1,996,750 ;(15 b) \$ 9,304,870 ;(15 c) \$ 96,888 ;(15 d) \$ 10,375 ;(15) \$ 11,408,883 ;(16)$ $\$ 141,640 ;$; (17a) $\$ 7,235,117$; (17b) $\$ 10,881,716$; (17c) $\$ 164,613$; (17) $\$ 18,281,446 ;(19) \$ 1,217,853 ;(20) \$ 31,049,822 ;(21) \$ 310,937 ;(22) \$ 6,423,159 ;$ (23a) \$29,142; (23b) \$671,852; (24) \$613,804; (25) \$196,210.

15d This expenditure has been considerably reduced by a credit received (École Polytechnique de Montréal).

17c Two branches of the University of Montreal library system reported salaries for library student assistants; however, in order for the totals to properly aggregate their responses have been marked NA/UA. The Ecole Polytechnique branch reported \$51,697 (CAD) and the HEC Montreal branch reported \$112,916 (CAD) in student assistant salaries.

23a A credit was received which was greater than the expenditures. The answer is in fact: 3,046 (École Polytechnique de Montréal).

26c Two branches of the University of Montreal library system reported library student assistants; however, in order for the figures to properly aggregate their responses have been marked UA/NA. The Ecole Polytechnique branch reported 1 student assistant and the HEC Montreal branch reported 2 student assistants.

\section{NATIONAL AGRICULTURAL LIBRARY}

All figures are as of 09/30/2008.

The Washington, DC, Reference Center is included.

1a Volumes held June 30, 2007 revised to 2,433,952.

18 Fringe benefits are included.

\section{NATIONAL LIBRARY OF MEDICINE}

All figures are as of 09/30/2008.

There are 27 service points at the National Library of Medicine (NLM) at the following NLM facilities: History of Medicine desk, circulation desk, reference desk, photocopy service desk, telephone service desk. 


\section{NATIONAL LIBRARY OF MEDICINE, cont.}

31 This figure represents onsite transactions only, and it is based on sampling.

32 These are internal circulations only. NLM does not circulate materials outside of the Library-- only on ILL.

\section{NEBRASKA}

$1 \mathrm{a}$

$1 b$

$1 b i$

1 bii

2

4

4aii

$4 \mathrm{~b}$

4biii

13

$15 a$

$15 c-15 d$

16

\section{NEW MEXICO}

The following university libraries are included: Zimmerman, Centennial, Parish, and Fine Arts/Design, Tireman Education, Bainbridge Visual Resources, and the Native American Studies libraries.

Library branches not included: Gallup Campus Branch Library, Los Alamos Campus Branch Library, Taos Campus Branch Library, Valencia Campus Branch Library. Volumes held June 30, 2007 revised to 3,167,709.

Due to budget cuts our materials budget has been slashed; therefore, the result would be fewer materials were acquired or purchased.

Budget cuts account for less monies available to purchase of materials, especially monographs.

Because of physical space needs within the libraries there has been more emphasis on weeding. In addition, more emphasis has been placed on database maintenance and one result is that more volumes being withdrawn than in the past.

Due to budget cuts, the monographs have been severely slashed to make up for the serials costs which are clearly out of pace with our materials budget.

Because our materials budget has been slashed we fully expect these figures will continue to decrease.

Despite the budget cuts, we continue to purchase more serials as needed.

Decrease is due to the cancellation of print from one publisher that provides a large number of content.

In 2007-2008 we have embarked on a large project to claim/inactivate titles; this project may account for the decrease in titles received.

Faculty seemed more inclined to use film and video more in the classroom; as a result the library is purchasing more and more of these items to support the curriculum. This appears to be a trend.

Monograph purchases have reduced significantly due to a change in our approval plan and due to the stress that serials place on the remaining materials budget. Increasingly, more money is devoted to serials while monograph purchases decrease. our budget.

Less funds have been devoted to contract binding because we are purchasing less print serials. This appears to be a trend.

The libraries has been canceling print subscriptions in lieu of electronic serials; monies were used from other sources to increase support in this area.

One major reason for this decrease is the struggle to keep pace with inflation. As a result, ongoing electronic resource purchases will most likely decrease.

This significant drop can be attributed to the way in which the data were collected in 2006-2007. The person who supplied the data in previous years, included everything purchased through the Nebraska Library Commission (even if UNL Libraries used its own funds) and therefore the amount for 2006-2007 was about \$455,670. For 2007-2008, only items that were paid for by the Nebraska Library Commission were included, so now we have a lower figure. If the databases we paid for had been included in 2007-2008, then the figure would have been closer to the amount listed in 2006-2007.

2006-2007 statistics represent a year that was uncharacteristically low. 2007-2008 is actually a more consistent number for these purchases.

The services points have not increased at UNL; however, the way we count them has changed. We now count the Scholarly Communications, Student Union, Geology Reference, and Math Reference desks as service points.

The increase in instruction is due to demand; more faculty are requesting instruction.

The increase is due to class size; more instruction was offered to larger classes.

This appears to be a trend; the reference transactions are decreasing annually. We can speculate that our users increasingly rely on online search engines (Google) for some information that was formerly acquired from the reference desk.

This increase is the reduction of acquisitions; we are not buying as much so we have to get the items from other libraries. Secondly, our marketing has improved. We have been promoting our interlibrary loan services more widely. The fact that full-time instructional faculty has increased may also explain this increase.

Library branches included: Geology, Music, C. Y. Thompson, Architecture, Engineering and Mathematics libraries. 


\section{NEW MEXICO, cont.}

\section{NEW YORK}

1a

$1 b i$

1bii

Serial counts nearly doubled due to the following factors: 1) $\$ 1,827,541$ in insurance funds were used to replace fire-damaged materials including databases with full text. The library was able to purchase more titles than were lost in the fire. 2) The library was able to purchase bundled packages through special consortial arrangements, resulting in the acquisition of additional journals. 3) Coding improvements allowed for more accurate counts of all journals including government documents. 4) The library conducted a project to replace print journals with electronic versions, when possible.

This increase is related to the purchase/licensing by the University of New Mexico's (UNM) slide library of 28,000 digital image files along with their annual cataloging of 37,753 images.

2007-2008 count increased due to a special purchase of a local vendor's video inventory.

$\$ 1,827,541$ in insurance funds were used to replace fire-damaged materials including databases with full text.

The UNM slide library made significant, one-time investments in licensing and electronic resources (ARTstor and other digital image purchases and licensing). This was possible through expending unused/vacant salary lines.

All UNM libraries increased their student budgets significantly in part due to increases in minimum wage.

One-time electronic resource purchases increased by $38 \%$ because of the following two reasons: 1) The UNM slide library made significant, one-time investments in licensing and electronic resources (ARTstor and other digital image purchases and licensing). This was possible through expending unused/vacant salary lines. 2) $\$ 1,827,541$ in insurance funds was used to replace firedamaged materials including databases with full text.

All UNM libraries increased their on-going electronic resource purchases. This appears to be a national trend. University Libraries also conducted a project to replace print journals with electronic versions, when possible.

All figures are as of 08/31/2008.

Library branches included: Bobst Library; Institute of Fine Arts (IFA) Library; Real Estate Institute (REI) Library; Courant Institute of Mathematical Sciences (CIMS) Library; Institute for the Study of the Ancient World (ISAW) Library; Law School Library; Medical School and Dental School Libraries.

Volumes held June 30, 2007 revised to 4,988,264. The number of e-books in 2006-2007 was 503,764 and increased to 537,022 in 20072008 , thereby increasing the volumes held at the beginning of the year by 33,258.

2006-2007's gross volumes added count was incorrect and excluded some volumes processed by the Acquisitions department.

Previous years' withdrawals were inflated by weeding projects implemented to move materials offsite that resulted in a large number of withdrawals. No such project was launched in 2007-2008 because of time restraints caused by the 2007-2008 ILS migration.

In the 2007-2008 year, the monographs department was not fully staffed, which resulted in fewer processing of orders. Additionally, monograph orders were not processed for two months of the year during the implementation phase of our recent ILS migration.

In 2007-2008 a concerted effort was made to provide more access to free serials via SFX as compared to prior years.

In 2007-2008 there was a decrease in orders due to the ILS migration creating a temporary slow down in the ordering workflow. In 2007-2008 manuscript purchases increased from $\$ 62,000$ to $\$ 236,000$.

Increased vendor costs for offsite storage in 2007-2008; increased spending on library refurbishment projects.

Major purchasing of one-time resources was focused more on electronic materials in 2007-2008.

The increase in ongoing e-resources purchases in 2007-2008 is a reflection of the continued transition in serials purchases from print to electronic.

Computer hardware/software costs went up in 2007-2008 due to an ILS system migration and corresponding vendor costs.

The ILL service for users increased from 2006-2007 to 2007-2008 and fees increased correspondingly (OCLC ShaRes fees increased in $2007-2008$ by $\$ 31,000)$.

The decrease in 2007-2008 represents a general trend of decreasing in-person reference service.

The decrease in circulations in 2007-2008 was caused by a decrease in renewals due to increasing the circulation period for M.A. students, our heaviest renewers.

Better exposure of records due to improved electronic resources; our institution is a member of RAPID consortium, which has allowed quicker fulfillment of orders.

\section{NEW YORK PUBLIC LIBRARY}

Fringe benefits are included. 


\section{NEW YORK STATE LIBRARY}

All figures are as of 03/31/2008.

\section{NORTH CAROLINA}

Library branches included: University Library, Health Sciences Library, Institute of Marine Sciences Library and the Law Library.

1

\section{NORTH CAROLINA STATE}

Includes 6.3 FTE professionals and support staff on special grant funds; 5.4 FTE on special projects; excludes 3.25 FTE paid from funds contributed by Duke, North Carolina State, and North Carolina Central Universities for support of the Triangle Research Libraries Network.

Volumes held June 30, 2007 revised to 6,412,105. Updated to reflect 272,901 e-books cataloged in 2007-2008. Health Sciences Library reduced by 15,161 volumes to correct flawed count in 2006-2007. is available. E-books added in 2007-2008 have been added to (1a) volumes held previous year.

Journal titles de-duped using ISSN as unique identifier. Titles without ISSNs were excluded entirely in order to eliminate items of a non-serial nature. This has likely also excluded some serial records lacking an ISSN, resulting in a possible undercount. purchased in 2007-2008 added about 5,200 titles. Includes: Springer Online Journal Archive (1,146), JSTOR packages (641), American Periodicals Series (1,520), America's Newspapers (1,380), Alt-Press Watch (206), and several smaller packages. In addition, more records were included in 2007-2008 because data was pulled from Serials Solutions; 2006-2007 report was based on local system, which has a higher percentage of records that lack ISSN.

Includes payments for CRL memberships.

Includes $\$ 5,908,862$ in grants, trust and other special funds (w/out benefits); excludes $\$ 399,192$ in expenditures for the Triangle Research Libraries Network from funds contributed by Duke, North Carolina State and North Carolina Central universities. Includes approx. \$900,000 expended for compact shelving during 2007-2008. Special funding for salary increases to reflect new state classifications also contributed to overall increase.

Library branches included: Design, Natural Resources, Textiles, Veterinary Medicine; African-American Cultural Center Reading Room, and the College of Education Media Center library.

1

Includes 401,497 e-books.

2 Includes some monographic standing order items paid from serials funds.

15a-15b Some monographic titles are paid from serials funds included in (15b).

15d Includes Bibliographic Utilities (\$176,002), Document Delivery $(\$ 143,756)$, and Association memberships (\$217,084).

\section{NORTHWESTERN}

All figures are as of 08/31/2008.

Library branches included: Includes Main Library, Deering Library, Seeley G. Mudd Science and Engineering Library, Math Library, Geology Library, and Schaffner Library (Chicago campus).

1-2 Includes government documents.

4b Law and Health Science serials data unavailable.

6, 11, 25 Health Science figures unavailable.

37 Northwestern reports number of fields in which PhDs were awarded annually, not total number of fields in which PhDs could be awarded.

\section{NOTRE DAME}

Library branches included: Hesburgh Library (Main) includes: Architecture Library, Art Image Library, Business Information Center, Chemistry/Physics Library, Engineering Library, Kellogg/Kroc Information Center, Mathematics Library, Life Sciences Library (closed 7/1/2008). In addition the Kresge Law Library and University Archives are included.

The number of serials titles currently received but not purchased $(17,070)$ is composed of (4bi) consortial: 3,976; (4bii) freely accessible: 9,611; (4biii) print: 82 and (4biv) government documents: 3,401. These responses were changed to UA/NA so that the operating system could aggregate other figures from the Notre Dame Law library survey.

This figure represents our entire University Archives holdings. It consists primarily of archival records and manuscripts but it also includes substantial quantities of printed, microfilm, artifact, audio, visual, film, graphic, and digital materials. Our holdings are organized according to the archival principle of provenance. Our controls over our records are therefore primarily oriented towards the creator of the records and only secondarily on the particular types of materials. 


\section{NOTRE DAME, cont.}

11 Graphic materials housed in the Art Image Library consists of two components: the analog and digital collections. The analog slide collection holds approximately 210,000-230,000 35mm slides available to faculty and students for research, teaching and presentation purposes. The digital collection has over 31,000 digital images. However, due to copyright restrictions, these low resolution images are only available to Art, Art History and Design faculty and are not included in the total.

OHIO

Includes Athens (main), Chillicothe, Lancaster, Southern, Eastern, Zanesville libraries as well as the College of Osteopathic Medicine Learning Resource Center.

$1 \quad$ Decreased after past volume count records were audited. See footnote (1a) for more detail.

1a Volumes held June 30, 2007 revised to 2,841,899. Decreased by 331,000 after audit of past volume count records. Increased by 259,367 to reflect e-books added as part of a large one-time purchase (LexisNexis U.S. Serials Set). Total change is a decrease of 71,633.

6, 8 Decreased after past item count records were audited.

9 Linear footage reassessed in 2007-2008.

10 Decreased after past item count records were audited.

11 Increase reflects transfer of slide library from College of Fine Arts to University Libraries, and acquisition of 2000 ceremonial paintings.

12-13 Decreased after past item count records were audited.

17a-17b Decrease due to eight positions moved from 'professional staff' category to 'support staff' category.

26 Overall reduction in numbers due to campus-wide budget cuts and to increase in minimum wage for student assistants.

26a Decrease reflects overall reduction of ten positions; an additional eight positions were moved from 'professional staff' category to 'support staff' category.

26b Increase reflects addition of eight positions moved from 'professional staff' category to 'support staff' category, reduction of one support staff position.

26c Decrease in number of students employed due to increase of minimum wage.

34 Large decrease due to change in OhioLINK's algorithm for distributing patron-initiated borrowing requests among member institutions.

38 Large increase reflects fulfillment of goal in university's strategic plan.

\section{OHIO STATE}

Include the following branch libraries: Ackerman; Architecture; Biological Sciences/Pharmacy; Business; Cartoon Research; Children's Hospital; Health Sciences; Fine Arts; Food, Agriculture and Environmental Sciences; Law; Music/Dance; Science and Engineering; Sullivant; Theatre Research Institute; University Archives; Veterinary Medicine.

Excludes: Agricultural Technical Institute; Ohio Agricultural Research and Development Center; Regional Campus Libraries at Lima, Mansfield, Marion, and Newark.

Includes 311,182 e-books: ACLS Humanities (1,590); Early American Imprints (75,461); Ebrary-leased (7,224); English Short Title Catalog (136,425); Health Sciences Library Collection (10,468); HeinOnline (382); Lyell Collection (294); Making of Modern Law (21,809); NetLibrary (15,227): Oxford Reference (267): Safari (2,825): Single titles/locally digitized (4,456); Wright American Fiction $(2,839)$.

1a Volumes held June 30, 2007 revised to 6,244,095.

2 Includes 326 purchased e-books, primarily published by Ebrary.

13 Audio, film, video data combined; cannot be separated.

15d Includes additional Ohionet bills not reflected during previous year (2006-2007).

29-30 Instructional activity did not decline. Data in 2006-2007 was over-reported.

$38 \quad$ Excludes research and clinical faculty.

41-42 Includes professional students.

\section{OKLAHOMA}

1a Volumes held June 30, 2007 revised to 5,047,646.

1b Increased purchases of e-books.

1bi Increased de-selection projects. 


\section{OKLAHOMA, cont.}

4a-4ai Inclusion of Law Integrating Resources. Serials statistics are reported as de-duped for all libraries.

4aii-4biv Serials statistics are reported as de-duped for all libraries following ARL Best Practices.

16 Increased preservation activities.

19 Figure reflects change in reporting methods.

22 Data demonstrates change in collection development practices.

$23 \mathrm{~b} \quad$ Due to change in reporting methods.

24 Additional hardware and software purchased.

\section{OKLAHOMA STATE}

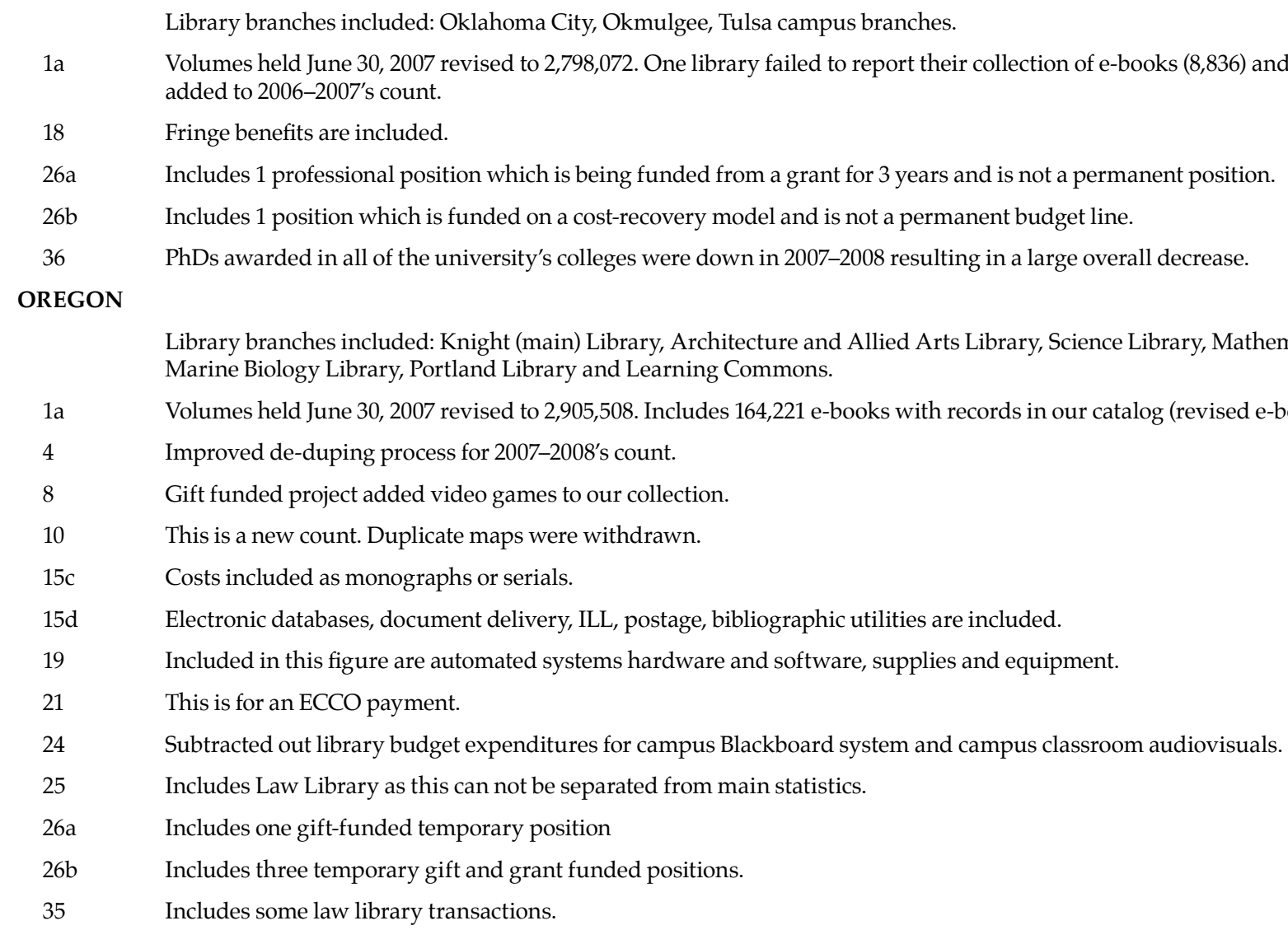

\section{PENNSYLVANIA}

1a Volumes held June 30, 2007 revised to 5,993,082.

16 The figures reported in question (16) was overstated due to an error, which will be corrected in a subsequent reporting period.

\section{PENNSYLVANIA STATE}
Survey includes all University Park Libraries, as well as the Campus branch libraries. Campus branch Library locations are as
follows: Abington, Altoona, Behrend, Berks, Beaver, Capital, Brandywine, DuBois, Fayette, Great Valley, Hazelton, Lehigh Va follows: Abington, Altoona, Behrend, Berks, Beaver, Capital, Brandywine, DuBois, Fayette, Great Valley, Hazelton, Lehigh
Mont Alto, McKeesport, New Kensington, Schuylkill, Shenango Valley, Wilkes-Barre, Worthington-Scranton, and York.
1a Volumes held June 30, 2007 revised to 5,221,673.
1bi-1bii Reported per format.
4aii The increase from 2006-2007 was primarily attributed to a separate count of titles for 2007-2008 received on memberships (consortia) or as packages.
4biii The 2006-2007 survey included government documents, which the 2007-2008 survey "broke out" into a separate line. Also enhancements to our records permitted us to include "children" records in the purchased line in 2007-2008 instead of in the received, but not purchased line of 2006-2007. 


\section{PENNSYLVANIA STATE, cont.}

10

21

22

25

\section{PITTSBURGH}

Includes the Barco Law Library, and branch campuses in Bradford, Greensburg, Johnstown, and Titusville. Health Science libraries include the Falk Library of the Health Sciences, the Western Psychiatric Institute and Clinic Library, the Libraries at UPMC Shadyside and the University of Pittsburgh Cancer Institute.

1a

Volumes held June 30, 2007 revised to 5,505,919. Changed the starting total per ARL's instructions to include four large, one-time e-book collections added this year.

21 Includes expenditure for catalog support software.

\section{PRINCETON}

4

\section{PURDUE}

1

Library branches included: main library and ten additional branches.

Increased figures due to acquisition growth in electronic serial titles and larger packages with more titles.

More government documents are now reflected in Voyager. Over the past 5-6 years (2002-2008), Princeton has been cataloging the government documents that were not part of the OCLC conversion and the Marcive Retrospective conversion of congressional hearings.

Calculation represents both an increase in the shift from one-time to subscription status, but also represents a more accurate rendering of the respective statuses based on enhanced resource coding.

Includes the library system on the West Lafayette campus, consisting of an undergraduate library, 11 subject libraries, and an archives and special collections unit.

Includes cataloged government documents. Includes e-books from ASM handbooks: 22; CogNet: 451; Credo Reference: 340; EEBO: 125,000; ECCO: 150,000; EngNet Base: 105; Gale Virtual Reference Library: 90; History e-Book Project: 1,708; Knovel Online: 1,662; ASAE: 4; Safari Tech Books: 878; Springer C books: 11,821; Stat Ref: 12; ASAE text Books: 5; World Bank e-Library: 1,957; and others totaling 295,034 .

Increase in net volumes added reflects fewer gross items added and fewer items withdrawn than in 2006-2007.

Represents number of titles rather than volumes. Excludes e-books and materials purchased on sponsored research programs.

Excludes numbered monograph serials.

Titles were deduped as indicated by instructions.

Includes cataloged depository, exchange, and gift serials, and e-journals received through statewide consortium. Cannot disaggregate consortial, freely accessible, or government documents at this time.

Decrease from 2006-2007 is in part due to change in reporting to more accurately reflect monograph expenditures.

Represents bibliographic and full text databases for which expenditures can be tracked separately. Expenditures for other databases, and for materials such as maps and audio visuals, are included in those for monographs (15a) and serials (15b) and cannot be disaggregated.

Reflects expenditures for bibliographic utilities $(176,264)$ previously reported in other operating expenditures.

Fringe benefits are included.

Decrease in ILL costs due to new membership in RAPID resource sharing in September 2007.

Increase from 2006-2007 reflects new positions added.

Excludes use of online, interactive tutorial "CORE".

Includes 2,227 reference transactions via digital reference service. 
Main library complex: Stauffer- Humanities and Social Sciences; Douglas- Engineering Science; WD Jordan- Special Collections/ Music; Education. Law and Bracken Health Sciences libraries are included (except where otherwise stated).

All figures are for our fiscal year ending April 20, 2008 (except where otherwise stated).

4

4ai

6

15-25

$1 \mathrm{a}$

$1 b$

9

11

13

$15 d$

17

\section{ROCHESTER}

Includes the Rush Rhees Library and the Sibley Music Library.

1a

2

3

4bii

5

$15 \mathrm{c}$

18

19

24

\section{RUTGERS}

4ai

These statistics are for the entire library system except for Law. \$159,302; (23b) \$101,377; (24) \$369,915; (25) \$30,828.

Manuscripts and archives are reported in cubic feet.

Corrected 2006-2007 total is 12,496.

One FTE professional and 1 FTE student grant-funded.

Does not include continuations. Includes 66 e-books.

Includes 76 government documents.

Freely accessible count includes 76 government documents.

Rush Rhees backfiles not included. salaries of other library employees.

Includes Gleason Library renovation project.

Includes IMLS grant and Gleason Library renovation project.

5,753 of these transactions are virtual.

Includes reserves.

Includes Sibley Music Library and Edward G Miner Library.

Includes Sibley Music Library and Edward G. Miner Library.

Includes Sibley Music Library and Edward G. Miner Library.
These statistics are for the entire library system. Serial titles are not broken out by branch or discipline.

We acquired more purchases primarily through CRKN (Canadian Research Knowledge Network).

Expenditures as reported in Canadian dollars: (15a) \$1,646,522; (15b) \$7,442,031; (15c) \$183,297; (15d) \$367,831; (15) \$9,639,681; (16) $\$ 86,377$; (17a) $\$ 3,449,078$; (17b) \$4,734,509; (17c) \$318,562; (17) \$8,502,149; (19) \$1,517,236; (20) \$19,745,443; (21) \$0; (22) \$5,729,117; (23a)

Library branches included: Fondren Library, Brown Fine Arts Library, and the Business Information Center.

Includes 24,271 Texshare e-books; 37,189 government document e-books; and 1,216 Netlibrary and other e-books.

Includes 7,024 government document e-books and 446 non-document e-books.

Corrected 2006-2007 total is 34,200.

Includes memberships (\$209,870.54), ILL (\$34,990.67), in-house binding supplies $(\$ 14,279.02)$, and bibliographic utilities (\$139,966.63).

Volumes held June 30, 2007 revised to 3,674,165. Added 1,189 lecture notes in computer science volumes.

Includes a physical count of 2,406 volumes purchased by the Sibley Music Library.

Sibley Music Library salaries and wages in the amount of $\$ 751,433$ do not include fringe benefits. Fringe benefits are included in the

Sibley Music Library reports are based upon based on sampling (120 of the reported total).

Sibley Music Library reports are based upon based on sampling (2,600 of the reported total).

Includes Sibley Music Library and Edward G. Miner Library. The number of full-time instructional faculty in 2007-2008 was determined by using the Integrated Postsecondary Education Data System (IPEDS).

Includes Sibley Music Library and Edward G. Miner Library. Includes 415 full-time medical students.

Includes Sibley Music Library and Edward G. Miner Library. Includes 415 full-time medical students.

Includes two law libraries, services to a non-Rutgers medical school and campuses in Camden, Newark, and New Brunswick.

Increase due to format changes to online journals and access to new databases. 


\section{RUTGERS, cont.}

4biv 2006-2007 report did not include government documents in print format.

10-13 Audiovisual Materials figures cannot be disaggregated. Total audiovisual is 152,216 for main library. Total audiovisual count for Main, Camden Law \& Newark Law is 153,475.

21 2006-2007 figure misreported: correct figure was \$22,644.

22 Increase attributable to inflation, considerable format changes to online, and new databases.

\section{SASKATCHEWAN}

Includes Education, Engineering, Natural Sciences, and Veterinary Medicine branch libraries.

Excludes Theological College libraries on campus that are not part of the library system.

1a Volumes held June 30, 2007 revised to 2,016,709.

1bii $\quad$ Figures decreased because of a major weed of collection in 2006-2007.

2-7 Government documents are included in these figures.

15-25 Expenditures as reported in Canadian dollars: (15a) \$2,152,080; (15b) \$6,411,407; (15c) \$0; (15d) $\$ 468,994 ;(15) \$ 9,032,481 ;(16) \$ 73,493$; (17a) $\$ 4,180,970 ;$; (17b) $\$ 3,254,716$; (17c) $\$ 279,388 ;$ (17) $\$ 7,715,074 ;(19) \$ 1,261,326 ;(20) \$ 18,082,374 ;(21) \$ 33,550 ;(22) \$ 5,564,082 ;(23 a)$ $\$ 102,779 ;$; (23b) \$0; (24) \$343,638; (25) \$93,511.

15b Correction to 2006-2007 figure: should be $\$ 8,679,015$.

17 Computer programmers, and budget officers included in professional staff.

20 Includes funding from grants and special funds.

22 Includes $\$ 917,969$ in provincial funding for resources for the Saskatchewan Health Information Resources Partnership; unable to determine exact portion of this funding that would be specific to the $U$ of Saskatchewan.

38 Includes professors, associate professors, assistant professors, lecturers and instructors.

39 Includes 45 certificate/diploma students.

$40 \quad$ Includes 702 certificate/diploma students.

$41 \quad$ Includes 263 post-graduate clinical students.

\section{SMITHSONIAN}

All figures are as of 09/30/2008.

Library branches included: Smithsonian Institution (SI) Libraries has a total of 20 libraries located throughout the museums and research institutes of the Smithsonian Institution. Included are: National Air and Space Museum Library, Washington, DC; National Museum of American History, Washington, DC; National Museum of Natural History Library, Washington, DC; National Postal Museum Library, Washington, DC; National Zoological Park Library, Washington, DC; Smithsonian American Art Museum, National Portrait Gallery Library, Washington, DC; Smithsonian Environmental Research Center Library, Edgewater, Maryland; Anacostia Museum and Center for African American History and Culture Library, Washington, DC; Botany and Horticulture Library, Washington, DC; Cooper-Hewitt National Design Museum Library, New York, New York; Dibner Library of the History of Science and Technology, Washington, DC; Earl S. Tupper Library Smithsonian Tropical Research Institute, Republic of Panama; Freer Gallery of Art and Arthur M. Sackler Gallery Library, Washington, DC; Hirshhorn Museum and Sculpture Garden Library, Washington, DC; John Wesley Powell Library of Anthropology, Washington, DC; Joseph F. Cullman, 3rd Library of Natural History, Washington, DC; Museum Studies and Reference Library, Washington, DC; Museum Support Center Library, Suitland, Maryland; Vine Deloria Jr. Library, National Museum of the American Indian, Suitland, Maryland; Warren M. Robbins Library, National Museum of African Art, Washington DC.

1a Volumes held June 30, 2007 revised to 1,229,903.

3 2007-2008, SI Libraries' senior management approved changing the method of counting number of physical volumes held to include all items that have barcodes and item records - not title-level. This includes all serials (bound \& unbound) that have a barcode attached, and anything else that we barcode.

9 Number of manuscripts added is estimated at 0.08 linear feet, so rounded this is zero. In $2006-2007=2,124+0=2,124$.

15a-15b Smithsonian Institution Libraries serves as the purchasing agent for all monographs, serials and electronic resources for the Smithsonian Institution. Purchases processed for The Woodrow Wilson International Center for Scholars (which is not under SIL administration) are not added to the SIL collections and the expenditures for monographs and serials are not included in (15). Includes costs for OCLC bibliographic utilities only. This cost was previously reported in (19)- Other operating expenditures. 


\section{SMITHSONIAN, cont.}

There was significant turnover of staff in 2006-2007, and several "high cost" positions were filled at a lower salary. However, SI libraries were also able to fill several professional positions that had been vacant for several years, resulting in a large increase in salary figures for professionals. These are actual "on board" costs, and do not include any "lapsed salaries" due to vacancies. Lapsed salaries were reprogrammed for expenditures in other areas.

Includes staff travel, training, cataloging, digitizing, collection-care supplies and services, general, preservation and automation supplies, equipment purchases, memberships, equipment maintenance, and miscellaneous contracts. It does not include health and transit benefits, building maintenance, public programs, exhibitions and development expenditures.

Through an internal grant from a large endowment, SI was able to purchase 90 back years (1900-1989) of Science Citation Index. Includes only OCLC bibliographic utility costs.

24 Total includes OCLC license and fees for Illiad (ILL) software. This was a new cost for 2007-2008.

26c Student assistants (Scholtz then O'Hara) equal 0.30 FTE which is rounded to zero.

28 In years prior to 2007-2008 the number of weekly public service hours was calculated based on 31 service points open for 8 hours per day equaling 248 hours. Per this year's (2007-2008) instructions, 31 of our service points are open 8 hours per day (9am - 5 pm) resulting in access during a 40 hour week. The 32nd service point is open 3 days per week for 8 hours, which should not change the result.

$35 \quad$ Borrows are steadily increasing as our funding levels decrease.

\section{SOUTH CAROLINA}

1a Volumes held June 30, 2007 revised to 3,581,962.

4a-4b The Main Library did not report numbers for questions (4a-4b) in 2006-2007, so the total number was artificially low in $2006-2007$.

\section{SOUTHERN CALIFORNIA}

Library branches included: USC Libraries, Norris Medical Library, Jennifer Ann Wilson Dental Library \& Learning Center, and the Asa V. Call Law Library.

1a Volumes held June 30, 2007 revised to 4,569,723.

3 Asa V. Call Law Library count is bibliographic.

15 2006-2007 corrected figures sent to ARL on 01/27/09 (published/originally reported figures for main campus only, excluding Law \& Medicine): (15) \$9,994,720; (15a): \$2,087,891; (15b): \$4,417,035; (15c): \$3,474,921; (15d): \$14,873; (21): \$708,552; (22): \$2,227,031. Method: (15a) derived completely from ASRs, both regular \& special funds; (15b) derived as 8\% less than 1(5b) for 2007-2008 as otherwise figure is too high to be realistic using method for 2007-2008; differential added to (15c); (15c) derived Lana spreadsheet total and other expenses derived from ASRs; (15d) derived completely from ASRs, both regular \& special funds; (21) \& (22) derived Lana spreadsheet totals.

38 Includes 1,483 full-time medical faculty.

\section{SOUTHERN ILLINOIS}

2 Includes e-books: Springer Book series archive (4,516 volumes) and Contemporary Literary Criticism (252 volumes).

4b The increase reflects improved tracking of free e-journals by Ex Libris SFX (our e-journal management system).

\section{SUNY-ALBANY}

All university libraries are included.

1a Volumes held June 30, 2007 revised to 2,150,165.

1bi Count includes 49,298 e-books. This large increase over the 6,313 reported in 2006-2007 is due to improved means of counting e-books.

$2 \quad$ Includes 1,136 e-books.

12-13 These figures were recounted.

16 Excludes theses and dissertations.

19 The figures reported here are much lower than what was reported during the last survey period (2006-2007). These numbers reflect our best efforts to capture other operating expenditures, but are not comprehensive due to internal accounting difficulties.

25 Includes bibliographic utility fees.

33 Circulation in 2006-2007 was published as 90,160 but has been revised to 142,963.

\section{SUNY-BUFFALO}

23b, 25 This information is unavailable. 


\section{SUNY-BUFFALO, cont.}

30 An additional 2,805 students completed the Library Skills Workbook in 2007-2008.

\section{SUNY-STONY BROOK}

Marine and Atmospheric Science Information Center; Math, Physics, Astronomy; Music; Chemistry; Science and Engineering; Stony Brook Southampton Campus Libraries are included.

1a Volumes held June 30, 2007 revised to 2,240,762.

15

$15 d$

16

\section{SYRACUSE}

The Law Library is included.

1bii Library continues to withdraw duplicate copies of monographs and print format of titles when available in electronic format.

24 Data was unavailable in 2006-2007.

Figure for 2006-2007 was incorrectly reported.

Science, Engineering, and Architecture Library (SEAL), Charles L. Blockson Afro-American Collection, Ambler Library, Tyler School of Art Library, and the Harrisburg Library are included.

Volumes held June 30, 2007 revised to 3,137,616.

Increase due to more precise calculation of total expenditures related to interlibrary loan services.

Decrease from 2006-2007 due to significant reduction of student assistant FTE, and to an error in support staff FTE reported in 2006-2007.

TENNESSEE

Support staff of 78 reported for 2006-2007 was incorrect.

Significant reduction in student assistant FTE.

Includes Main (University Libraries-Knoxville and UT Space Institute-Tullahoma); Health Sciences Libraries (Health Sciences Library-Memphis, Preston Medical Library, and the Knoxville part of College of Medicine in Memphis); and the College of Law Library.

1a Volumes held June 30, 2007 revised to 3,252,763.

Before de-duplication: Main Titles Purchased: Electronic 28,078, Print 3,605, Total 31,683.

Before de-duplication: Main Titles Not Purchased: 15,268.

As part of the Media Center reorganization, 804 of 984 slide sets were withdrawn during 2007-2008.

2006-2007 hours should have been 148; medical hours were counted twice. The University Libraries are open 148 hours and the University of Tennessee Space Institute (UTSI) Library is open 40 hours, but the UTSI hours occurred within the hours of operation for the University Libraries.

In 2006-2007 the UT Health Sciences PhD programs were omitted.

All figures are as of 08/31/2008.

Includes the University of Texas (Austin) Libraries, the Briscoe Center for American History, the Tarlton Law Library, and the Humanities Research Center.

$1 \quad$ Volumes held June 30, 2007 revised to 10,103,429.

$4 \quad$ We de-duped our SFX list by ISSN, resulting in a list of electronic serials by unique ISSNs. The list of electronic serials by unique ISSN was compared to the list of active print/other serial titles derived from the Innovative Interfaces Serials Control module. ISSNs which appeared in both lists were dropped from the print/other list, resulting in a list of print/other titles with unique ISSNs or with no ISSNs at all. Both lists (electronic and print/other) were then sorted into purchased/ non-purchased.

15 Includes total expenditures for library materials of $\$ 14,007,642$ by the University Libraries; $\$ 96,055$ by the Center for American History; $\$ 1,463,540$ by the Tarlton Law Library; and $\$ 2,047,254$ by the Humanities Research center. 
TEXAS, cont.

30

36

Includes users of online tutorials.

Does not include EdD degrees.

\section{TEXAS A\&M}

All figures are as of 08/31/2008.

Library branches included: Sterling C. Evans Library, Library Annex, Cushing Memorial Library, West Campus Library, Policy Sciences and Economics Library, Medical Sciences Library, Technical Reference Center (Architecture Library), Jack K. Williams Library (Galveston), Texas A\&M University at Qatar Library.

1bi Increase reflects correction in volume count over the past years (prior to 2007-2008). Have new computer algorithm for counting volumes that indicated previous years were under-reported.

2 Decrease in "Numbers of monographs Purchased" result of not purchasing as many e-books as in previous 2 years (20052006/2006-2007)

4 Change in serials reported reflects change in programming algorithm used to count the various categories of purchased and not purchased serials. New algorithm reflects better identification and coding of unique serial titles.

4biv Government Documents are reflected in freely accessible figure.

12 Increase in Audio materials reflects new commitment to acquiring materials in this formal. Growth for 2007-2008 is approximately the same as growth between 2006-2007 and 2005-2006.

\section{TEXAS TECH}

All figures are as of 08/31/2008.

Library branches included: Architecture Library, Museum Library, Geosciences Library, Vietnam Archives Library, Southwest Collections/Special Collections Library, Harrington Library Texas Tech University Health Science Center (TTUHSC)/Amarillo, Montes-Gallo Library of the Health Sciences TTUHSC/El Paso, Library of the Health Sciences TTUHSC/Odessa, Preston Smith HSC Library, and the Law Library.

\section{TORONTO}

\section{All figures are as of 04/30/2008.}

Includes the Main Libraries, Campus and Federated \& Affiliated Libraries: Architecture, Astronomy, Business, Chemistry, Criminology, Dentistry, Earth Sciences, Engineering \& Computer Sciences, Fine Art, Industrial Relations, Information Studies Forum, Innis College, Knox College, Law, Massey, Music, New College, Physics, Inst. of Policy Analysis, Regis College, Royal Ontario Museum, St. Augustine Seminary, St. Michael's College, Trinity College, University College, U of Toronto Schools, Victoria College. Branch Campuses libraries include the U of Toronto- Mississauga, and U of Toronto-Scarborough. Hospital Libraries: Baycrest, Bloorview Kids Rehab, Mt. Sinai Hospital, St. Michael's Hospital, Toronto Rehab.

1a Volumes held 30 June 2007 revised to 10,953,627. This is to account for e-books not counted in 2006-2007.

4a There was an error in de-duping serials in 2006-2007.

15-25 Expenditures as reported in Canadian dollars: (15a) $\$ 8,433,993 ;(15 b) \$ 11,609,207 ;(15 c) \$ 6,040,015 ;(15 d) \$ 0 ;(15) \$ 26,083,215 ;(16)$ $\$ 318,976$; (17a) $\$ 15,503,557 ;$ (17b) \$16,472,975; (17c) \$4,045,115; (17) \$36,021,647; (19) \$6,419,062; (20) \$68,842,900; (21) \$1,735,232; (22) $\$ 7,641,745$; (23a) \$727,318; (23b) \$0; (24) \$1,993,548; (25) \$98,680.

Number of service points reported in 2006-2007 was inaccurate.

\section{TULANE}

Included: Turchin Business Library and the Nadine Vorhoff Library for the Newcomb Center for Research on Women.

1a Volumes held June 30, 2007 revised to 3,146,953.

\section{VANDERBILT}

1 Includes 460,617 e-books held by library system.

1a Volumes held June 30, 2007 revised to 3,393,519. Total includes 81,854 electronic books purchased during 2007-2008.

1bi Per ARL instructions, removed 81,854 e-books purchased during the year (2007-2008) and added them to the total in (1a).

\section{VIRGINIA}

Library branches included: Alderman (main), Astronomy, Biology/Psychology, Brown Science \& Engineering, Chemistry, Clemons Undergraduate, Education, Fiske Kimball Fine Arts, Math, Music, Physics, and Small Special Collections.

1

Includes 348,171 e-books. The Darden Graduate Business Library does not own e-books.

$1 b i$ 


\section{VIRGINIA, cont.}

1bii 0 e-books withdrawn.

2318,393 e-books purchased.

4 The serials figures for all University of Virginia libraries are reported in the University Library (Main) survey only (see 2007-2008 ARL Statistics).

4b This is a large increase (49\%) over what was reported in 2006-2007. Unpaid electronic serials holdings were significantly undercounted in 2006-2007 and previous years.

17 The University of Virginia is excluding fringe benefits from salary expenditures beginning with 2007-2008.

17c For the University of Virginia Library, the "student assistant" category includes part-time temporary and wage workers.

36-42 Reported for all University of Virginia libraries on the University Library (main) survey.

\section{WASHINGTON}

Includes main campus library, UW-Bothwell library and the UW-Tacoma library.

1a Volumes held June 30, 2007 revised to 7,304,356.

The number of current serials titles received is 61,979 and is composed of: (4a) Serials titles currently purchased: 33,582; subdivided to (4ai) electronic: 22,297 and (4aii) print: 11,285 (4b) 24,383 Serials titles currently received but not purchased: (4bi) Consortial: 0; (4bii) Freely Accessible: 12,812; (4biii) Print (and other format): 3,766 and (4biv): Government Documents: 7,805. Both (4a) and (4b) and their subtotals were changed to UA/NA so that the operating system could aggregate the 4,014 serials reported in the Washington Law library survey.

\section{WASHINGTON STATE}

Includes WSU-Vancouver, WSU-Tri-Cities, WSU Energy Library, WSU Spokane, and WSU Intercollegiate College of Nursing (ICN). During the year (2007-2008) a merger was in progress between WSU-Spokane and ICN.

1a Volumes held June 30, 2007 revised to 2,294,937.

1b Includes 10,678 e-books.

4 The change from the figure reported for 2006-2007 is largely due to improvements in procedures for title identification and deduplication. The 2006-2007 figure is under review.

15a Includes some media expenditures reported in past years (prior to 2007-2008) under (15c).

15c Some media expenditures reported here in past years (prior to 2007-2008) are now included in (15a).

15d Copyright fees; $\$ 4,295$; computing services: $\$ 12$; collections software: $\$ 24,288$; document delivery: $\$ 6,000$; consortia dues and related expenditures: $\$ 68,099$; other/purpose not reported: $\$ 107,857$.

26a Includes 2.5 FTE temporary positions.

33 Total circulations for 2006-2007 were misreported as 253,118. The correct total was 320,418.

34 Figure for 2006-2007 was misreported. The correct figure is 53,739.

35 Figure for 2006-2007 was misreported. The correct figure is $24,374$.

\section{WASHINGTON U.-ST. LOUIS}

1bi Includes 4,350 electronic books added in main library this year (2007-2008).

4ai This number is significantly higher than previous years (prior to 2007-2008) because we used the count of electronic serials provided by Serials Solutions. We are unable to dedupe for the names or correlate them with III.

19 Fringe benefits are included in other expenditures.

\section{WATERLOO}

All figures are as of 04/30/2008.

Library branches included: Includes the Musagetes Architecture Library, Optometry Learning Resource Centre, and the TriUniversity Storage Annex (University of Waterloo materials only).

1a, 1bi Volumes held 30, June 2007 revised to 2,259,482.

4ai Change from using in-house ERM system to SFX knowledgebase.

4aii Figure changed due to counting titles rather than counting orders (subscriptions).

4bi Included in (4ai).

4biii Included in (4aii). 


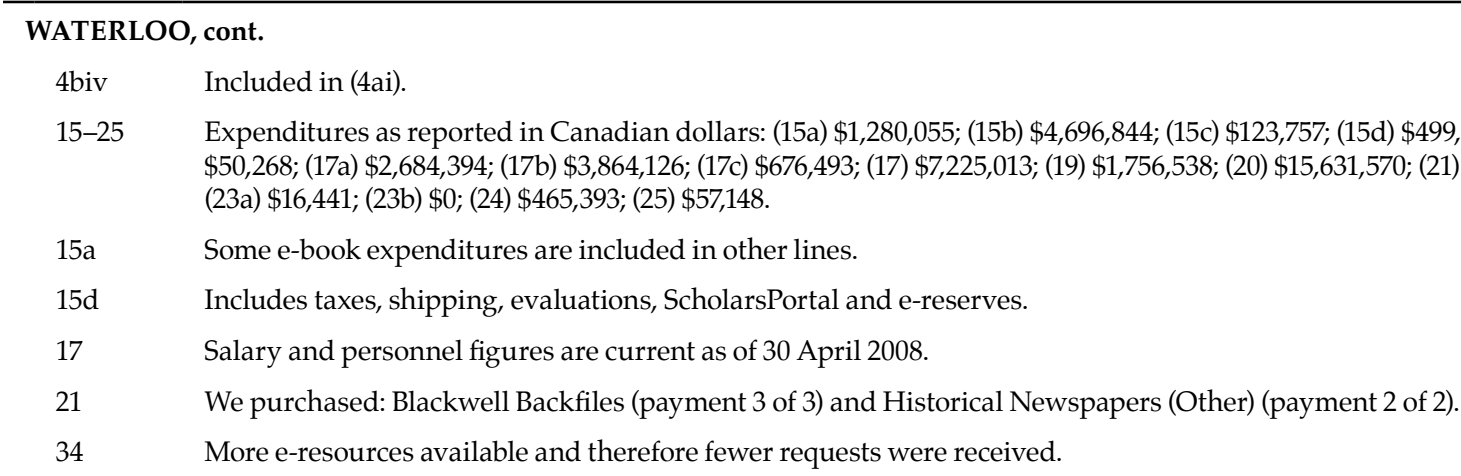

\section{WAYNE STATE}

All figures are as of 09/30/2008.

Includes the Reuther Archives of Labor and Urban Affairs, the Shiffman Medical Library, and the Neef Law Library. The Medical and Law library statistics cannot be disaggregated from the main statistics because the Medical and Law collections are an integral part of the main library.

1a Volumes held June 30, 2007 revised to 3,658,639. The number of volumes held at the beginning of the year has been adjusted to reflect 82,607 e-books previously uncounted and 117,259 volumes of the U.S. Serials Set acquired from Lexis Nexis in 2007-2008.

1 bi The number of volumes added gross includes 6,870 e-books acquired from Ebrary, Safari, Knovel, Netlibrary, SourceOECD, Making of Modern Law, Hein, Access Medicine, Lange e-books, and other vendors.

2 The number of monograph volumes purchased includes 6,870 e-books acquired from Ebrary, Safari, Knovel, Netlibrary, SourceOECD, Making of Modern Law, Hein, Access Medicine, Lange e-books, and other vendors. Counts from years prior to 20072008 have excluded e-books.

9 This is an estimated figure.

34-35 We refined the gathering of ILL statistics in 2007-2008. We now believe there was an over count in previous reports. The increase in 2007-2008 is due to a new article delivery service.

36 Includes EdD degrees.

\section{WESTERN ONTARIO}

All figures are as of 04/30/2008.

Library branches included: Archives and Research Collections Centre, C.B. (Bud) Johnston Library, Education Library, John \& Dotsa Bitove Family Law Library, Music Library, Allyn \& Betty Taylor Library, and the D.B. Weldon Library.

1a Volumes held June 30, 2007 revised to 3,497,689. Includes 135,611 e-books added in 2007-2008.

2 Number includes total 4,874 e-books: Electronic Book Library 555; Lippincott Wilson \& Wilson 623; Myilibrary 326; Springer 3,370.

4ai Includes Law Library as well. E-serials are centrally funded and made accessible for all locations.

9 Western Archives collection only in the Archives and Research Collections Centre.

10 Western Archives cartographic materials only in the Archives and Research Collections Centre.

11 London Free Press Photographic Negatives Collection in Western Archives, Archives and Research Collections Centre.

12 Music Library audio materials housed in Music and Archives and Research Collections Centre.

13 Music video materials housed in the Music Library and the Archives and Research Collections Centre.

15-25 Expenditures as reported in Canadian dollars: (15a) \$2,289,750; (15b) \$9,221,541; (15c) \$0; (15d) $\$ 244,132 ;(15) \$ 11,755,423 ;(16) \$ 117,338$; (17a) $\$ 4,427,387$; (17b) $\$ 3,985,251$; (17c) $\$ 303,508$; (17) \$8,716,146; (19) \$944,120; (20) \$21,533,027; (21) \$56,130; (22) $\$ 6,992,523 ;(23 a) \$ 187,691$; (23b) \$0; (24) \$345,320; (25) \$12,183.

15b E-serials centrally funded and include Law Library.

15d, 21 This is centrally funded and includes Law Library.

22 E-resources centrally funded and include Law Library.

23a-25 Centrally funded and includes Law Library.

35 Central service for all library locations including Law Library. 
Library branches included: General Library System (GLS): Archives, Art, Astronomy, Biology, Business, Chemistry, College (Undergraduate library), Geography, Geology \& Geophysics, Math, Memorial, Music, Physics, Social Science, Social Work, Special Collections, Agriculture and Life Sciences (Steenbock), Map, Merit (formerly CIMC Education), Primate, School of Library \& Information Studies (SLIS), Wendt Engineering, Health Sciences (Ebling), Law, and the Wisconsin Historical Society Library.

1

There is an increase of 105,000 volumes added from the Wisconsin Historical Society Library (WHS), our University's library for American History. These items represent the WHS share of total number of titles $(220,000)$ of pre-1976 publications from the purchased MARCIVE GPO download that occurred in 2006-2007.

Volumes held June 30, 2007 revised to 7,933,636.

The count of e-books added jumped from the 17,914 previously reported to a new figure of 23,884 . This is because the earlier count was based on the bibliography records being coded as format "am" (language monographs). However, in the process of investigating how to count e-books we discovered that some vendors are coding their records as either "aa" (language mono component part) or "ad" (language mono subunit).

Our campus has undergone efforts to ensure we have a reliable count of serials held based upon analyzing records within our local cataloging systems. This new methodology involved a comparison of records in two key databases in which records are captured for serials (SFX and Voyager). This process involved efforts to eliminate duplicate titles across all campus libraries through several verification levels. The process is not flawless but represents our best efforts at determining and tracking a reliable serial account for our campus.

This figure is a representative sample of reference transactions based upon an annual collection period. Specifically, the most recent statistics-gathering period was held in March 2008. Approximately, 35 campus libraries participated, resulting in 2,640 documented reference questions over a period of 7 days. This figure $(2,640)$ does not include directional, technical, or "other" questions as defined by campus libraries.

Library branches included: Arts Library, Bass Library, Beinecke Rare Book and Manuscript Library, Chemistry Library, Classics Library, Divinity Library, Drama Library, Engineering and Applied Science Library, Epidemiology \& Public Health Library, Forestry and Environmental Studies Library, Geology Library, Kline Science Library, Law Library, Lewis Walpole Library, Mathematics Library, Medical Library, MUDD Library, Music Library, Social Science Library, and Information Services.

The change in purchased serial titles is at best an estimate and the figure reported 2006-2007 may be in error but may also reflect the change in instructions for reporting at the title rather than the subscription level. This estimate in serial titles has resulted in an increase in number of items but an reduction in average price paid. Thus, our unit price for serials purchased may be unrealistically low.

This is included in serials print counts.

Since 2002-2003 we report the number added only in the notes field; count of computer files added in 2007-2008 is 3,282. We are not reporting the cumulative figures.

An estimate in serial titles has resulted in an increase in number of items but an reduction in average price paid. Thus, our unit price for serials purchased may be unrealistically low. See footnote for (4a).

In 2007-2008 a number of Yale libraries reopened after renovations were completed.

Due to duplicate entries initial circulation count for 2006-2007 should have been reported as 604,828 and the total circulation count including renewals should have been 967,707 .

Numbers includes Law library.

Information concerning doctoral degrees taken from the IPEDS Survey of Degrees granted. First professional degrees are not included.

First professional fields not included.

Number represents full-time "ladder" faculty at the University.

All figures are as of 04/30/2008.

Library branches included: Bronfman Business Library; Steacie Science and Engineering Library; Frost Library; Scott Library and the Law Library.

Volumes held June 30, 2007 revised to 3,102,024. Includes 9,4342 e-books not included in gross volumes added.

We have 1,217 linear feet of photographs in our archives. If 110 prints equals one inch, that means we have 1,606,693 pieces.

Expenditures as reported in Canadian dollars: (15a) \$2,603,949; (15b) \$7,960,761; (15c) \$211,229; (15d) \$104,393; (15) \$10,880,332;

(16) $\$ 179,715$; (17a) $\$ 5,832,338 ;(17 b) \$ 5,144,542 ;$ (17) $\$ 1,075,888 ;(17) \$ 12,052,768 ;(19) \$ 3,817,569 ;(20) \$ 26,930,384 ;(21) \$ 1,668,127 ;(22)$

$\$ 4,508,763$; (23a) \$77,399; (23b) \$0; (24) \$275,542; (25) \$24,667. 
QUESTION FoOTNOTE

NUMBER

YORK, cont.

15d Reporting YBP costs as instructed. 


\section{ARL Member Libraries as of January 1, 2009}

The Association of Research Libraries (ARL) represents the interests of 123 libraries that serve major North American research institutions. The ARL Statistics and Measurement program is organized around identifying, collecting, analyzing, and distributing quantifiable information describing the characteristics of research libraries.

\begin{tabular}{|c|c|c|}
\hline Institution & Category & Full Name of Institution \\
\hline Alabama & $\mathrm{S}$ & University of Alabama \\
\hline Alberta & $\mathrm{C}$ & University of Alberta \\
\hline Arizona & S & University of Arizona \\
\hline Arizona State & S & Arizona State University \\
\hline Auburn & $S$ & Auburn University \\
\hline Boston & $\mathrm{P}$ & Boston University \\
\hline Boston College & $\mathrm{P}$ & Boston College \\
\hline Brigham Young & $\mathrm{P}$ & Brigham Young University \\
\hline British Columbia & $\mathrm{C}$ & University of British Columbia \\
\hline Brown & $\mathrm{P}$ & Brown University \\
\hline Berkeley, California & $\mathrm{S}$ & University of California, Berkeley \\
\hline California, Davis & $\mathrm{S}$ & University of California, Davis \\
\hline California, Irvine & $\mathrm{S}$ & University of California, Irvine \\
\hline California, Los Angeles & S & University of California, Los Angeles \\
\hline California, Riverside & $\mathrm{S}$ & University of California, Riverside \\
\hline California, San Diego & S & University of California, San Diego \\
\hline California, Santa Barbara & $\mathrm{S}$ & University of California, Santa Barbara \\
\hline Case Western Reserve & $\mathrm{P}$ & Case Western Reserve University \\
\hline Chicago & $\mathrm{P}$ & University of Chicago \\
\hline Cincinnati & $S$ & University of Cincinnati \\
\hline Colorado & $\mathrm{S}$ & University of Colorado \\
\hline Colorado State & $\mathrm{S}$ & Colorado State University \\
\hline Columbia & $\mathrm{P}$ & Columbia University \\
\hline Connecticut & S & University of Connecticut \\
\hline Cornell & $\mathrm{P}$ & Cornell University \\
\hline Dartmouth & $\mathrm{P}$ & Dartmouth College \\
\hline Delaware & S & University of Delaware \\
\hline Duke & $\mathrm{P}$ & Duke University \\
\hline Emory & $\mathrm{P}$ & Emory University \\
\hline Florida & S & University of Florida \\
\hline Florida State & S & Florida State University \\
\hline George Washington & $\mathrm{P}$ & George Washington University \\
\hline Georgetown & $\mathrm{P}$ & Georgetown University \\
\hline Georgia & S & University of Georgia \\
\hline Georgia Tech & S & Georgia Institute of Technology \\
\hline Guelph & $\mathrm{C}$ & University of Guelph \\
\hline Harvard & $\mathrm{P}$ & Harvard University \\
\hline Hawaii & S & University of Hawaii \\
\hline Houston & $S$ & University of Houston \\
\hline
\end{tabular}

\section{Location}

Tuscaloosa, Alabama

Edmonton, Alberta

Tucson, Arizona

Tempe, Arizona

Auburn, Alabama

Boston, Massachusetts

Boston, Massachusetts

Provo, Utah

Vancouver, British Columbia

Providence, Rhode Island

California, Berkeley

Davis, California

Irvine, California

Los Angeles, California

Riverside, California

La Jolla, California

Santa Barbara, California

Cleveland, Ohio

Chicago, Illinois

Cincinnati, Ohio

Boulder, Colorado

Fort Collins, Colorado

New York, New York

Storrs, Connecticut

Ithaca, New York

Hanover, New Hampshire

Newark, Delaware

Durham, North Carolina

Atlanta, Georgia

Gainesville, Florida

Tallahassee, Florida

Washington, DC

Washington, DC

Athens, Georgia

Atlanta, Georgia

Guelph, Ontario

Cambridge, Massachusetts

Honolulu, Hawaii

Houston, Texas 


\section{Institution}

Howard

Illinois, Chicago

Illinois, Urbana

Indiana

Iowa

Iowa State

Johns Hopkins

Kansas

Kent State

Kentucky

Laval

Louisiana State

Louisville

McGill

McMaster

Manitoba

Maryland

Massachusetts

MIT

Miami

Michigan

Michigan State

Minnesota

Missouri

Montreal

Nebraska

New Mexico

New York

North Carolina

North Carolina State

Northwestern

Notre Dame

Ohio

Ohio State

Oklahoma

Oklahoma State

Oregon

Pennsylvania

Pennsylvania State

Pittsburgh

Princeton

Purdue

Queen's

Rice

\section{Category Full Name of Institution}

P Howard University

S University of Illinois at Chicago

S University of Illinois at Urbana

S Indiana University

S University of Iowa

$S \quad$ Iowa State University

P Johns Hopkins University

S University of Kansas

S Kent State University

S University of Kentucky

C Laval University

S Louisiana State University

S University of Louisville

C McGill University

C McMaster University

C University of Manitoba

S University of Maryland

S University of Massachusetts

P Massachusetts Institute of Technology

P $\quad$ University of Miami

S University of Michigan

S Michigan State University

S University of Minnesota

$S \quad$ University of Missouri

C University of Montreal

S University of Nebraska-Lincoln

S University of New Mexico

P New York University

S University of North Carolina

S North Carolina State University

P Northwestern University

P University of Notre Dame

S Ohio University

S Ohio State University

S University of Oklahoma

S Oklahoma State University

$S \quad$ University of Oregon

P University of Pennsylvania

S Pennsylvania State University

S University of Pittsburgh

P Princeton University

S Purdue University

C Queen's University

P Rice University

\section{Location}

Washington, DC

Chicago, Illinois

Urbana, Illinois

Bloomington, Indiana

Iowa City, Iowa

Ames, Iowa

Baltimore, Maryland

Lawrence, Kansas

Kent, Ohio

Lexington, Kentucky

Quebec, Quebec

Baton Rouge, Louisiana

Louisville, Kentucky

Montreal, Quebec

Hamilton, Ontario

Winnipeg, Manitoba

College Park, Maryland

Amherst, Massachusetts

Cambridge, Massachusetts

Coral Gables, Florida

Ann Arbor, Michigan

East Lansing, Michigan

Minneapolis, Minnesota

Columbia, Missouri

Montreal, Quebec

Lincoln, Nebraska

Albuquerque, New Mexico

New York, New York

Chapel Hill, North Carolina

Raleigh, North Carolina

Evanston, Illinois

Notre Dame, Indiana

Athens, Ohio

Columbus, Ohio

Norman, Oklahoma

Stillwater, Oklahoma

Eugene, Oregon

Philadelphia, Pennsylvania

University Park, Pennsylvania

Pittsburgh, Pennsylvania

Princeton, New Jersey

West Lafayette, Indiana

Kingston, Ontario

Houston, Texas 


\begin{tabular}{|c|c|c|c|}
\hline Institution & Category & Full Name of Institution & Location \\
\hline Rochester & $\mathrm{P}$ & University of Rochester & Rochester, New York \\
\hline Rutgers & S & Rutgers University & New Brunswick, New Jersey \\
\hline Saskatchewan & $\mathrm{C}$ & University of Saskatchewan & Saskatoon, Saskatchewan \\
\hline South Carolina & S & University of South Carolina & Columbia, South Carolina \\
\hline Southern California & $\mathrm{P}$ & University of Southern California & Los Angeles, California \\
\hline Southern Illinois & $\mathrm{S}$ & Southern Illinois University & Carbondale, Illinois \\
\hline SUNY-Albany & S & University at Albany, State University of New York & Albany, New York \\
\hline SUNY-Buffalo & $S$ & University at Buffalo, State University of New York & Buffalo, New York \\
\hline SUNY-Stony Brook & $\mathrm{S}$ & State University of New York at Stony Brook & Stony Brook, New York \\
\hline Syracuse & $\mathrm{P}$ & Syracuse University & Syracuse, New York \\
\hline Temple & S & Temple University & Philadelphia, Pennsylvania \\
\hline Tennessee & S & University of Tennessee & Knoxville, Tennessee \\
\hline Texas & S & University of Texas & Austin, Texas \\
\hline Texas A\&M & S & Texas A\&M University & College Station, Texas \\
\hline Texas Tech & S & Texas Tech University & Lubbock, Texas \\
\hline Toronto & $\mathrm{C}$ & University of Toronto & Toronto, Ontario \\
\hline Tulane & $\mathrm{P}$ & Tulane University & New Orleans, Louisiana \\
\hline Utah & S & University of Utah & Salt Lake City, Utah \\
\hline Vanderbilt & $\mathrm{P}$ & Vanderbilt University & Nashville, Tennessee \\
\hline Virginia & S & University of Virginia & Charlottesville, Virginia \\
\hline Virginia Tech & S & Virginia Polytechnic Institute \& State University & Blacksburg, Virginia \\
\hline Washington & S & University of Washington & Seattle, Washington \\
\hline Washington State & S & Washington State University & Pullman, Washington \\
\hline Washington U.-St. Louis & $\mathrm{P}$ & Washington University & St. Louis, Missouri \\
\hline Waterloo & $\mathrm{C}$ & University of Waterloo & Waterloo, Ontario \\
\hline Wayne State & $S$ & Wayne State University & Detroit, Michigan \\
\hline Western Ontario & $\mathrm{C}$ & University of Western Ontario & London, Ontario \\
\hline Wisconsin & S & University of Wisconsin & Madison, Wisconsin \\
\hline Yale & $\mathrm{P}$ & Yale University & New Haven, Connecticut \\
\hline York & $\mathrm{C}$ & York University & North York, Ontario \\
\hline Boston Public Library & $\mathrm{N}$ & Boston Public Library & Boston, Massachusetts \\
\hline Canada Inst. SciTech Info. & $X$ & Canada Inst. for Scientific \& Technical Information & Ottawa, Ontario \\
\hline Center for Research Libraries & $\mathrm{N}$ & Center for Research Libraries & Chicago, Illinois \\
\hline Library of Congress & $\mathrm{N}$ & Library of Congress & Washington, DC \\
\hline National Agricultural Library & $\mathrm{N}$ & National Agricultural Library & Beltsville, Maryland \\
\hline Library and Archives Canada & $x$ & Library and Archives Canada & Ottawa, Ontario \\
\hline Naionat. Library of Medicine & $\mathrm{N}$ & National Library of Medicine & Bethesda, Maryland \\
\hline New York Public Library & $\mathrm{N}$ & New York Public Library & New York, New York \\
\hline New York State Library & $\mathrm{N}$ & New York State Library & Albany, New York \\
\hline Smithsonian Institution & $\mathrm{N}$ & Smithsonian Institution & Washington, DC \\
\hline $\begin{array}{l}\text { S: US public university } \\
\text { P: US private university } \\
\text { C: Canadian university } \\
\text { N: US nonuniversity } \\
\text { X: Canadian nonuniversity }\end{array}$ & & & \\
\hline
\end{tabular}





\section{BIBLIOGRAPHY}

\section{PART A: Selected Articles from the ARL Newsletter}

“The ARL Membership Criteria Index."ARL: A Bimonthly Newsletter of Research Library Issues and Actions 197 (April 1998$): 9$.

“Assessing ILL/DD Services: New Cost-Effective Alternatives." ARL: A Bimonthly Report on Research Library Issues and Actions from ARL, CNI, and SPARC 236 (October 2004): 9.

Askew Waller, Consuella, and Kaylyn Hipps. “Using LibQUAL+ and Developing a Culture of Assessment in Libraries.” ARL: A Bimonthly Report on Research Library Issues and Actions from ARL, CNI, and SPARC 221 (April 2002): 10-11.

Case, Mary M. "A Snapshot in Time: ARL Libraries and Electronic Journal Resources." ARL: A Bimonthly Report on Research Library Issues and Actions from ARL, CNI, and SPARC 235 (August 2004): 1-10.

. "The Impact of Serial Costs on Library Collections." ARL: A Bimonthly Report on Research Library Issues and Actions from ARL, CNI, and SPARC 218 (October 2001): 9.

Case, Mary M., and Judith Matz. "Framing the Issue: Open Access." ARL: A Bimonthly Report on Research Library Issues and Actions from ARL, CNI, and SPARC 226 (February 2003): 8-10.

Case, Mary M., and Prudence Adler. "Promoting Open Access." ARL: A Bimonthly Report on Research Library Issues and Actions from ARL, CNI, and SPARC 220 (February 2002): 1-5.

“Collections \& Access for the 21 ${ }^{\text {st }}$-Century Scholar: Changing Roles of Research Libraries." Report from the ARL Collections \& Access Issues Task Force. ARL: A Bimonthly Report on Research Library Issues and Actions from ARL, CNI, and SPARC 225 (December 2002).

Cook, Colleen, Fred Heath, and Bruce Thompson. "A Brief LibQUAL+ Phase One Progress Report." ARL: A Bimonthly Report on Research Library Issues and Actions from ARL, CNI, and SPARC 219 (December 2001): 7.

Crowe, William. "The End of History? Reflections on a Decade." ARL: A Bimonthly Report on Research Library Issues and Actions from ARL, CNI, and SPARC 226 (February 2003): 12-13.

Crow, Raym. "The Case for Institutional Repositories: A SPARC Position Paper." ARL: A Bimonthly Report on Research Library Issues and Actions from ARL, CNI, and SPARC 223 (August 2002): 1-4.

“The Future of Human Resources in Canadian Libraries." ARL: A Bimonthly Report on Research Library Issues and Actions from ARL, CNI, and SPARC 240 (June 2005): 9.

Grosetta Nardini, Holly. "Building a Culture of Assessment." ARL: A Bimonthly Report on Research Library Issues and Actions from ARL, CNI, and SPARC 218 (October 2001): 11.

Groves, Richard. "Sharing Best Practices by Disseminating Assessment Results via the Web." ARL: A Bimonthly Report on Research Library Issues and Actions from ARL, CNI, and SPARC 236 (October 2004): 6. 
Guedon, Jean-Claude. "Beyond Core Journals and Licenses: The Paths to Reform Scientific Publishing." ARL: A Bimonthly Report on Research Library Issues and Actions from ARL, CNI, and SPARC 218 (October 2001): 1-8.

Hahn, Karla. "The State of the Large Publisher Bundle: Findings from an ARL Member Survey." ARL: A Bimonthly Report on Research Library Issues and Actions from ARL, CNI, and SPARC 245 (April 2006).

Hipps, Kaylyn. "Diversity in the U.S. ARL Library Workforce." ARL: A Bimonthly Report on Research Library Issues and Actions from ARL, CNI, and SPARC 246 (June 2006): 1-2.

Johnson, Richard K. "Whither Competition?" ARL: A Bimonthly Report on Research Library Issues and Actions from ARL, CNI, and SPARC 217 (August 2001): 12-14.

Kyrillidou, Martha. "Reshaping ARL Statistics to Capture the New Environment." ARL: A Bimonthly Report on Research Library Issues and Actions from ARL, CNI, and SPARC 256 (February 2008): 9-11.

. "The Impact of Electronic Publishing on Tracking Research Library Investments in Serials." ARL: A Bimonthly Report on Research Library Issues and Actions from ARL, CNI, and SPARC 249 (December 2006): 6-7.

. "The Future of Librarians in the U.S. Workforce." ARL: A Bimonthly Report on Research Library Issues and Actions from ARL, CNI, and SPARC 246 (June 2006): 5.

. "ARL University Libraries' Spending Trends." ARL: A Bimonthly Report on Research Library Issues and Actions from ARL, CNI, and SPARC 242 (October 2005): 10.

"LibQUAL+ ${ }^{\mathrm{TM}}$ in 2004." ARL: A Bimonthly Report on Research Library Issues and Actions from ARL, CNI, and SPARC 236 (October 2004): 6.

. “Serials Trends Reflected in the ARL Statistics 2002-03." ARL: A Bimonthly Report on Research Library Issues and Actions from ARL, CNI, and SPARC 234 (June 2004): 14-15.

Kyrillidou, Martha, and William Crowe. "In Search of New Measures." ARL: A Bimonthly Newsletter of Research and Library Issues and Actions 197 (April 1998): 8-10.

Kyrillidou, Martha, and Kaylyn Hipps. "Symposium on Measuring Library Service Quality." ARL: A Bimonthly Report on Research Library Issues and Actions from ARL, CNI, and SPARC 215 (April 2001): 9-11.

Lippincott, Sarah, and Martha Kyrillidou. "How ARL University Communities Access Information: Highlights From LibQUAL+" ${ }^{\mathrm{TM}}$." ARL: A Bimonthly Report on Research Library Issues and Actions from ARL, CNI, and SPARC 236 (October 2004): 7-8.

Lynch, Clifford. "Institutional Repositories: Essential Infrastructure for Scholarship in the Digital Age." ARL: A Bimonthly Report on Research Library Issues and Actions from ARL, CNI, and SPARC 226 (February 2003): 1-7.

"Making Library Assessment Work." ARL: A Bimonthly Report on Research Library Issues and Actions from ARL, CNI, and SPARC 240 (June 2005): 9.

Shim, Wonsik "Jeff", Charles McClure, and John Carlo Bertot. "Measures and Statistics for Research Library Networked 
Services: ARL E-Metrics Phase II Report." ARL: A Bimonthly Report on Research Library Issues and Actions from ARL, CNI, and SPARC 219 (December 2001): 8-9.

Stuber, Peter. "Where does the Free Online Scholarship Movement Stand Today?" ARL: A Bimonthly Report on Research Library Issues and Actions from ARL, CNI, and SPARC 220 (February 2002): 9-15.

Unsworth, John M. "The Crisis in Scholarly Publishing in the Humanities." ARL: A Bimonthly Report on Research Library Issues and Actions from ARL, CNI, and SPARC 228 (June 2003): 1-4.

Wetzel, Karen, and Mary Jackson. "Portal Functionality Provided by ARL Libraries: Results of an ARL Survey." ARL: $A$ Bimonthly Report on Research Library Issues and Actions from ARL, CNI, and SPARC 222 (June 2002): 7-9.

Wilder, Stanley. "New Hires in Research Libraries: Demographic Trends and Hiring Priorities." ARL: A Bimonthly Report on Research Library Issues and Actions from ARL, CNI, and SPARC 221 (April 2002): 5-8.

Young, Mark. "ARL Salary Survey Highlights." ARL: A Bimonthly Report on Research Library Issues and Actions from ARL, CNI, and SPARC 246 (June 2006): 4.

\section{PART B: Related Books and Articles Published in Other Sources}

Arms, William. "Quality Control in Scholarly Publishing on the Web." The Journal of Electronic Publishing 8, no. 1 (August 2002).

Band, Jonathan. “The Three P's: A Tribute to Duane Webster." portal: Libraries and the Academy 9, no. 3 (2009): 367-74.

Baumol, W. J., and M. Marcus. Economics of Academic Libraries. Washington, DC: American Council of Education, 1973.

Bertot, John Carlo, Charles McClure, and Joe Ryan. Statistics and Performance Measures for Public Library Networked Services. Chicago: American Library Association, 2001.

Blixrud, Julia. "The Association of Research Libraries Statistics and Measurement Program: From Descriptive Data to Performance Measures." Proceedings from the $4^{\text {th }}$ Northumbria International Conference on Performance Measurement in Libraries and Information Services, edited by Joan Stein, Martha Kyrillidou, and Denise Davis. Washington, DC: Association of Research Libraries, 2002.

Branin, Joseph J. “Duane Webster's Contributions to Leadership Development in Research Libraries, 1970-2008.” portal: Libraries and the Academy 9, no. 3 (2009): 349-54.

Carlson, Scott. "The Deserted Library: As Students Work Online, Reading Rooms Empty Out - Leading Some Campuses to Add Starbucks." The Chronicle of Higher Education, November 16, 2001.

Case, Mary M. “Scholarly Communication: ARL as a Catalyst for Change." portal: Libraries and the Academy 9, no. 3 (2009): $381-96$.

Clapp, V. W. The Future of the Research Library. Urbana: University of Illinois Press, 1964.

Coffman, Steve. “Building Earth's Largest Library: Driving into the Future.” Searcher 7, no. 3 (March 1999). 
Competition Commission of the United Kingdom. Reed Elsevier, PLC, and Harcourt General, Inc.: A Report on the Proposed Merger, presented to Parliament by the Secretary of State and Trade and Industry by Command of Her Majesty, July 2001.

Cook, Colleen, Fred Heath, Martha Kyrillidou, and Duane Webster. "The Forging of Consensus: A Methodological Approach to Service Quality Assessment in Research Libraries - the LibQUAL+ Experience." Proceedings from the $4^{\text {th }}$ Northumbria International Conference on Performance Measurement in Libraries and Information Services, edited by Joan Stein, Martha Kyrillidou, and Denise Davis. Washington, DC: Association of Research Libraries, 2002.

Cook, Colleen, Fred Heath, and Bruce Thompson. "Score Norms for Improving Library Service Quality: A LibQUAL+ study." portal: Libraries and the Academy 2 (2002): 13-26.

Cook, Colleen, Fred Heath, Bruce Thompson, and Duane Webster. “LibQUAL+TM Preliminary Results from 2002.” Performance Measurement and Metrics 4, no. 1 (2003): 38-47.

Cook, Colleen, Fred Heath, Bruce Thompson, and Russell Thompson. The Search for New Measures: The ARL LibQUAL+Project-A Preliminary Report. Baltimore: Johns Hopkins University Press, 2001. Accessed at http:// muse.jhu.edu/demo/pla/1.1cook.html on August 22, 2001.

. “LibQUAL+: Service Quality Assessment in Research Libraries.” IFLA Journal 27 (2001): 264-68.

Council on Library and Information Resources. A Different Approach to the Evaluation of Research Libraries. Research Brief 6. Washington, DC: The Council, 1998.

Cronenwett, Philip N., Kevin Osborn, Samuel A. Streit, eds. Celebrating Research: Rare and Special Collections from the Membership of the Association of Research Libraries. Washington, DC: Association of Research of Libraries, 2007.

Crow, Raym. “The Case for Institutional Repositories: A SPARC Position Paper.” Washington, DC: Scholarly Publishing \& Academic Resources Coalition, 2002.

Cummings, Anthony M., et al. University Libraries and Scholarly Communication: A Study Prepared for The Andrew W. Mellon Foundation. Washington, DC: Association of Research Libraries, 1992.

Cummings, M. M. The Economics of Research Libraries. Washington, DC: Council on Library Resources, 1986.

Dewey, Barbara I. “The Imperative for Diversity: ARL's Progress and Role.” portal: Libraries and the Academy 9, no. 3 (2009): 355-62.

Downs, Robert B. “The Growth of Research Collections.” Library Trends: American Library History: 1876-1976 25 (July 1976): $55-80$. . “Uniform Statistics for Library Holdings.” Library Quarterly 16 (January 1946): 63-69.

Drake, Miriam A. “Forecasting Academic Library Growth.” College and Research Libraries 37 (January 1976): 53-59.

Franklin, Brinley, Colleen Cook, Martha Kyrillidou, and Bruce Thompson. "Library Investment Index—Why Is It Important?" Proceedings of the 2008 Library Assessment Conference: Building Effective, Sustainable, and Practical 
Assessment, August 4-7, 2008, Seattle, Washington. (Washington, DC: Association of Research Libraries, 2009): $147-54$.

Franklin, Brinley. “Duane Webster, Assessment Pioneer.” portal: Libraries and the Academy 9, no. 3 (2009): $339-48$.

Frazier, Kenneth. “The Librarians' Dilemma: Contemplating the Costs of the ‘Big Deal,,” D-Lib Magazine 7, no. 3 (March 2001), viewed online at http://www.dlib.org/dlib/march01/frazier/03frazier.html.

Funk, Cara J. "ARL and Association 3.0: Ten Management Challenges." portal: Libraries and the Academy 9, no. 3 (2009): 405-10.

Gooden, Paul, Matthew Owen, and Sarah Simon. Scientific Publishing: Knowledge is Power. New York: Morgan Stanley, 2002.

Guthrie, Kevin M. “It Didn't Have to Be This Way: Entrepreneurship at ARL During Duane Webster's Tenure.” portal: Libraries and the Academy 9, no. 3 (2009): 411-18.

Haslett, K. Mark. “Canada, Copyright, and the Common Good." portal: Libraries and the Academy 9, no. 3 (2009): 375-80.

Hayes, Robert M. Strategic Management for Academic Libraries: A Handbook. Westport, Conn.: Greenwood Press, 1993.

Heath, Fred. "A Salute to a Leader: ARL's Assessment Protocol Initiatives." portal: Libraries and the Academy 9, no. 3 (2009): 333-38.

Heath, Fred, Colleen Cook, Martha Kyrillidou, and Bruce Thompson. "ARL Index and Other Validity Correlates of LibQUAL+ Scores." portal: Libraries and the Academy 2 (2002): 27-42.

Hernon, Peter, and Robert E. Dugan. Action Plan for Outcomes Assessment in Your Library. Chicago: American Library Association, 2002.

Hernon, Peter, and John R. Whitman. Delivering Satisfaction and Service Quality. Chicago: American Library Association, 2001.

Hernon, Peter, and Ellen Altman. Assessing Service Quality. Chicago: American Library Association, 1998.

Hightower, Christy, Julie Sih, and Adam Tilgham. "Recommendations for Benchmarking Web Site Usage Among Academic Libraries." College and Research Libraries 59 (January 1998): 61-79.

Hiller, Steve, Martha Kyrillidou, and Jim Self, "Assessment in North American Research Libraries: a Preliminary Report Card." Performance Measurement and Metrics 7, no. 2 (2006): 100-6.

Jackson, Mary E. Measuring the Performance of Interlibrary Loan Operations in North American Research E College Libraries. Washington, DC: Association of Research of Libraries, 1998.

Jakubs, Deborah. “The Global Resources Program." portal: Libraries and the Academy 9, no. 3 (2009): 327-32.

Johnson, Richard K. "A Question of Access: SPARC, BioOne, and Society-Driven Electronic Publishing." D-Lib Magazine (May 2000), accessed at http://www.dlib.org/dlib/may00/johnson/05johnson.html on August 21, 2001. 
Johnson, Richard K, and Judy Luther. “The E-Only Tipping Point for Journals." Washington, DC: Association of Research of Libraries, 2007.

Kling, Rob, Lisa Spector, and Geoff McKim. “The Guild Model.” The Journal of Electronic Publishing 8, no. 1 (August 2002).

Kuhlman, A. F. “Two ARL Approaches to Counting Holdings of Research Libraries.” College and Research Libraries 21 (May 1960): 207-11.

Kyrillidou, Martha. "Library Assessment as a Collaborative Enterprise." Resource Sharing and Information Networks 1/2 (Special 2005-06 issue on the theme "Creative Collaborations: Libraries Within Their Institutions and Beyond"): 73-87.

. "From Input and Output Measures to Quality and Outcome Measures, or, from the User in the Life of the Library to the Library in the Life of the User." The Journal of Academic Librarianship 28, no. 1 (January-March 2002): $42-46$.

. “To Describe and Measure the Performance of North American Research Libraries.” IFLA Journal 27 (2001, No. 4): 257-63.

. "Research Library Trends: ARL Statistics." Washington, DC: Association of Research Libraries, 2001. Accessed at http://www.arl.org/stats/arlstat/jal99.html on August 22, 2001.

Kyrillidou, Martha, and Colleen Cook. “The Evolution of Measurement and Evaluation of Libraries: A Perspective from the Association of Research Libraries." Library Trends 56, no. 4 (Spring 2008): 888-909.

Kyrillidou, Martha, and Fred Heath, eds. “Measuring Service Quality." Library Trends 49 (Spring 2001).

Lakos, Amos. "Culture of Assessment as a Catalyst for Organizational Culture Change in Libraries." Proceedings from the $4^{\text {th }}$ Northumbria International Conference on Performance Measurement in Libraries and Information Services, edited by Joan Stein, Martha Kyrillidou, and Denise Davis. Washington, DC: Association of Research Libraries, 2002.

Lavoie, Brian, Lynn Silipigni Connaway, and Lorcan Demsey. "Anatomy of aggregate collections: the example of Google Print for libraries" D-Lib Magazine 11, no. 9 (September 2005): http://www.dlib.org/dlib/september05/ lavoie/09lavoie.html .

Lynch, Clifford A., and Joan K. Lippincott. "Institutional rEpository Deployment in the United States as of Early 2005." D-Lib Magazine 11, no. 9 (September 2005): http://www.dlib.org/dlib/september05/lynch/09lynch.html .

Mekkawi, Mod. “The ARL Library Index as a Decision-Making Tool." College and Research Libraries 43 (September 1982): 396-401.

Miller, Rush, and Sherrie Schmidt. “E-Metrics: Measures for Electronic Resources." Proceedings from the $4^{\text {th }}$ Northumbria International Conference on Performance Measurement in Libraries and Information Services, edited by Joan Stein, Martha Kyrillidou, and Denise Davis. Washington, DC: Association of Research Libraries, 2002.

Miller, Rush, Sherrie Schmidt, and Martha Kyrillidou. "New Initiatives in Performance Measures." Global Issues in 21 ${ }^{\text {st }}$ Century Research Librarianship, edited by Sigrun Klara Hannesdottir. Helsinki: NORDINFO, 2002. 
Molyneux, Robert E. "What Did Rider Do? An Inquiry into the Methodology of Fremont Rider's The Scholar and the Future of the Research Library." Libraries and Culture 29 (Summer 1994): 297-325.

. "Patterns, Processes of Growth, and the Projection of Library Size: A Critical Review of the Literature on Academic Library Growth." Library and Information Science Research 8 (January-March 1986): 5-28.

. The Gerould Statistics 1907/08 - 1961/62. Washington, DC: Association of Research Libraries, 1986.

Molyneux, Robert E., and Robert V. Williams. "Measuring the Internet." Annual Review of Information Science and Technology 34 (1999-2000): 287-339.

Morris, Dilys E., Collin B. Hobert, Lori Osmus, and Gregory Wool. “Cataloging Staff Costs Revisited.” LRTS 44 (2000): $70-83$.

Nitecki, Danuta, and Toni Olshen. "Developing Service Quality Measurement Skills Through an Online Course for Librarians." Proceedings from the $4^{\text {th }}$ Northumbria International Conference on Performance Measurement in Libraries and Information Services, edited by Joan Stein, Martha Kyrillidou, and Denise Davis. Washington, DC: Association of Research Libraries, 2002.

O'Connor, Lisa, Carolyn Radcliff, and Julie Gedeon. "Applying Systems Design and Item Response Theory to the Problem of Measuring Information Literacy Skills." College and Research Libraries 63, no. 6 (2002): 528-43.

Piternick, George. "ARL Statistics—Handle With Care." College and Research Libraries 38 (September 1977): 419-23.

."Library Growth and Academic Quality.” College and Research Libraries 24 (May 1963): 223-29.

Rider, Fremont. The Scholar and the Future of the Research Library. New York: Hadham Press, 1944.

Roche, Marilyn M. ARL/RLG Interlibrary Loan Cost Study. Washington, DC: Association of Research Libraries, 1993.

Rosenblatt, Susan. "Developing Performance Measures for Library Collections and Services." Chap. 18 in The Mirage of Continuity: Reconfiguring Academic Information Resources for the 21st Century, edited by Brian L. Hawkins and Patricia Battin. Washington, DC: Council on Library and Information Resources, 1998.

Shim, Wonsik, Charles McClure, and John Carlo Bertot. "Preliminary Statistics and Measures for ARL Libraries to Describe Electronic Resources and Services." Proceedings from the $4^{\text {th }}$ Northumbria International Conference on Performance Measurement in Libraries and Information Services, edited by Joan Stein, Martha Kyrillidou, and Denise Davis. Washington, DC: Association of Research Libraries, 2002.

Shulenburger, David. “The Political Climate for Higher Education During Duane Webster's ARL Tenure." portal: Libraries and the Academy 9, no. 3 (2009): 363-66.

Stubbs, Kendon. "Access and ARL Membership Criteria." Proceedings of the $125^{\text {th }}$ Meeting of the Association of Research Libraries (1993): 117-22.

. “University Libraries: Standards and Statistics.” College and Research Libraries 42 (November 1981): 527-38. “Apples and Oranges and ARL Statistics." Journal of Academic Librarianship 14 (September 1988): 231-35. 
. "On the ARL Library Index." Paper presented at the $108^{\text {th }}$ meeting of Research Libraries: Measurement, Management, Marketing (May 1986): Minneapolis, MN.

. “Lies, Damned Lies, ... and ARL Statistics?" Paper presented at the $108^{\text {th }}$ meeting of Research Libraries: Measurement, Management, Marketing (May 1986): Minneapolis, MN.

Sullivan, Maureen. “Duane Webster's Contribution to Organization Development in Academic and Research Libraries." portal: Libraries and the Academy 9, no. 3 (2009): 313-16.

Thompson, Bruce. "Some Alternative Quantitative Library Activity Descriptions/Statistics that Supplement the ARL Logarithmic Index." Washington, DC: Association of Research Libraries, 2006.

Thompson, Bruce, Colleen Cook, and Russell Thompson. "Reliability and Structure of LibQUAL+ Scores." portal: Libraries and the Academy 2 (2002): 3-12.

van Westrienen, Gerard, and Clifford A. Lynch. "Academic Institutional Repositories: dEployment Status in 13 Nations as of Mid 2005” D-Lib Magazine 11, no. 9 (September 2005): http://www.dlib.org/dlib/september05/ westrienen/09westrienen.html.

Vaughn, John C. "AAU and ARL: The Role of Partnerships and Collective Advocacy in Policy Development." portal: Libraries and the Academy 9, no. 3 (2009): 397-404.

Weiner, Sharon. “Library Quality and Impact: Is There a Relationship Between New Measures and Traditional Measures?" Journal of Academic Librarianship 31 (2005): 432-37.

West, Richard P. "The Coalition for Networked Information and the Rewards of Risk Taking." portal: Libraries and the Academy 9, no. 3 (2009): 317-25.

Wilder, Stanley J. The Age Demographics of Academic Librarians: A Profession Apart. A Report Based on Data from the ARL Annual Salary Survey. Washington, DC: Association of Research Libraries, 1995.

Williams, Robert V. “The Making of Statistics of National Scope on American Libraries, 1836-1986: Purposes, Problems, and Issues." Libraries and Culture 26 (Spring 1991): 464-85.

Wysocki, Bernard. “Scholarly Journals' Premiere Status is Diluted by Web.” Wall Street Journal, May 23, 2005; Page A1. 